\title{
Synthesis, Surface Modification and \\ Purification of Silicon and Germanium \\ Quantum Dots for Biological Applications
}

by

Amane Shiohara

A thesis

submitted to the Victoria University of Wellington in fulfilment of the requirements for the degree of

Doctor of Philosophy in Chemistry

Victoria University of Wellington July 2010 



\section{Abstract}

Quantum dots have applications in biomedical fields such as bio-imaging and drug delivery systems. This thesis describes research on silicon and germanium nanoparticles (quantum dots) synthesis and surface modification for biological applications. Purification methods of these quantum dots were also explored. In chapter 6 the application of silica nanoparticles into dry eye diagnosis was studied. The purpose of this research is to contribute the application of nanotechnology into biological fields. The crystalinity of the quantum dots was characterised by Transmission Electron Microscopy (TEM) and Selected Area Electron Diffraction analysis (SAED). The molecules on the surface of the quantum dots were characterised by Fourier Transform Infrared spectroscopy (FTIR) and Nuclear Magnetic Resonance (NMR).

Silicon quantum dots were synthesised with a microemulsion system and various types of molecules were attached on the surface of the silicon quantum dots. However, some of the capping molecules which have oxygen atoms tend to form bonds between oxygen and silicon. Therefore, in the later chapter (chapter 4) various chemical reactions were conducted on the molecules attached to the silicon quantum dots. The silicon quantum dots were capped with diene molecules and one of the double bonds was left on the terminal end. The terminal end double bonds were converted to the functional groups which contain oxygen atoms to form peptide bonds. In this way it was confirmed that it can reduce the risk of oxygen atoms to be attached on the surface of the silicon quantum dots. The molecules on the surface of the silicon quantum dots were characterised mainly by FTIR and ${ }^{1} \mathrm{H}$ NMR. Optical properties and cyto-toxicity of these silicon quantum dots were also measured and analysed depending on the surface molecules.

Two synthetic approaches were taken to produce germanium quantum dots. The first approach was the microemulsion system at room temperature. Different combinations of the surfactant and capping molecules were tested. For the second approach, high temperature bench top system was applied. In this method the bio-friendly molecules 
which have high boiling points were chosen as capping agents. The surface molecules were characterised by FTIR spectroscopy.

In chapter 6 the synthesis of dye molecules conjugated silica nanoparticles was described. The purpose of this research is to produce biologically safe nanoparticles which can be applied in dry eye diagnosis. Three different dyes were used to conjugate with the silica nanoparticles. Only fluorescein isothiocyanate (FITC) succeeded in conjugating with the nanoparticles. Optical properties of this sample were measured and compared with the free dye molecule. Also the sample was applied in human eyes to analyse the tear film layer.

An overall conclusion and future plans for the research were given in the last chapter. In this chapter, ideas of overcoming the problems and improving the techniques conducted in the research were described. 


\section{Acknowledgement}

During my $\mathrm{PhD}$ course, I have learned a lot of knowledge and skills of science and also non-science skills. There is a long list of people to whom I would like to show my appreciation. Without them this thesis would not have been completed and I would not have survived this hard time. In particular I would like to thank:

My supervisor Dr. Richard D. Tilley for encouraging me how to overcome the problems, advice and teaching me the amusingness of science.

My co-supervisor Associe professor Pter Northcote for his encouragement, advice, good humour, and supporting me in non-science way.

Professor Kenji Yamamoto for his unbelievable humour and his great help in Japan.

David Flynn for his help in TEM especially when it went down.

Dr. Teck H. Lin for his great advice, encouragement and being a very good friend in the team.

Dr. John Beal for his great advice, helping me in the lab, being a very good friend out of the lab and also proof reading my thesis to correct all my English mistakes.

Dr. John Watt and Sujay Prabakar for their great company, and being very good friends in many ways.

Dr. Kisten Edger and Dr. Conrad Lendrum for proof reading my thesis.

The "physicists” Dr. Jan Richter, Dr. Kirk Feindel, Dr. Antoine Lutti, and Bradley Douglass for their great company and help. 
Dr. Carla Fonseca and other colleagues within SCPS department for their great company.

My friends outside of university for their patience and support.

My family in Japan in particular my parents for understanding and huge support in mental and physical ways.

Lastly I would like to thank FRST IIOF scholarship and Curtis-Gordon scholarship, also JASCO scholarship for funding and financial support. 


\section{Table of Contents}

Chapter 1 Introduction

1-1. Motivation and general overview of the research .................................... 1

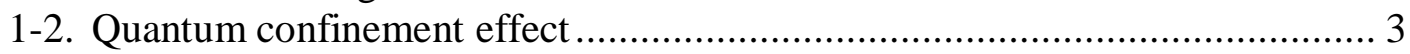

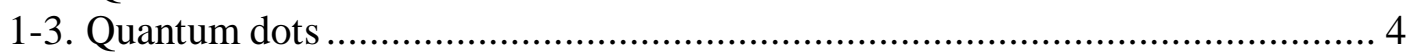

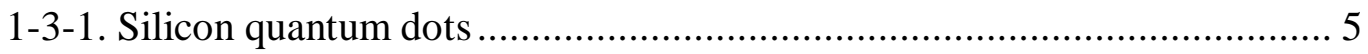

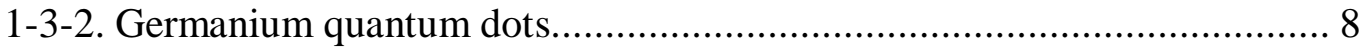

1-4. Representative syntheses of silicon quantum dots ................................... 9

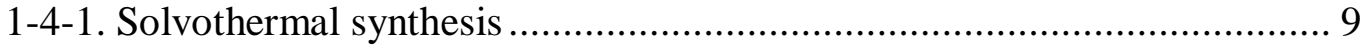

1-4-2. Microemulsion Synthesis............................................................... 10

1-5. Representative syntheses of germanium quantum dots............................... 12

1-6. Capping technique of quantum dots for biological applications.................... 14

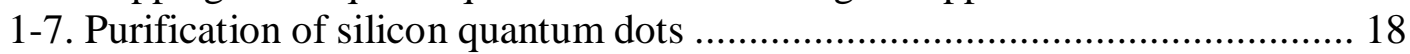

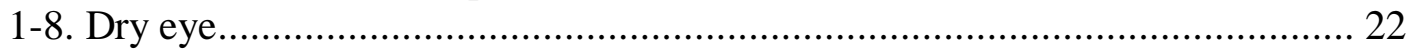

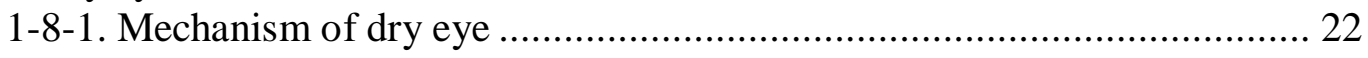

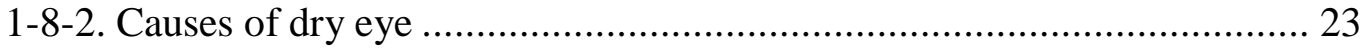

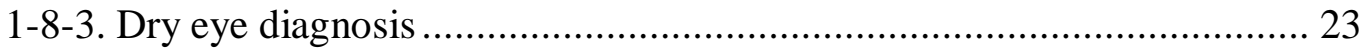

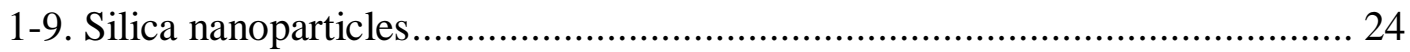

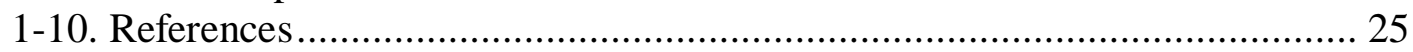

Chapter 2 Characterisation techniques..............................................................37

2-1. High Resolution Transmission Electron Microscopy (HRTEM)................... 37

2-1-1. Basic theory of TEM (Transmission Electron Microscopy).................... 37

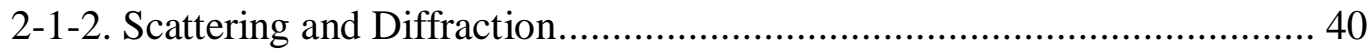

2-1-3. TEM image of crystalline nanoparticles.............................................. 43

2-2. Selected Area Electron Diffraction (SAED) ................................................ 44

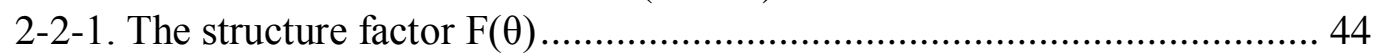

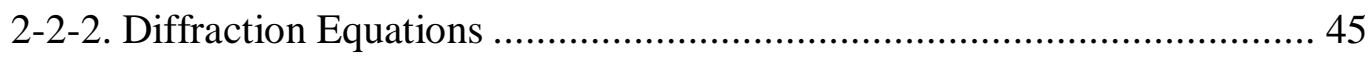

2-2-3. Ring patterns from polycrystalline materials .................................... 47

2-3. Energy Dispersive X-ray Spectrometry (EDS) …........................................ 49

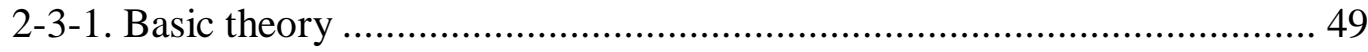

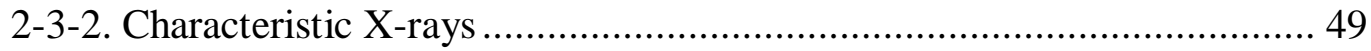

2-4. Fourier Transform Infrared spectroscopy (FTIR) ....................................... 51

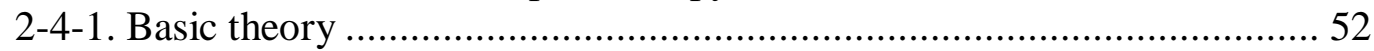

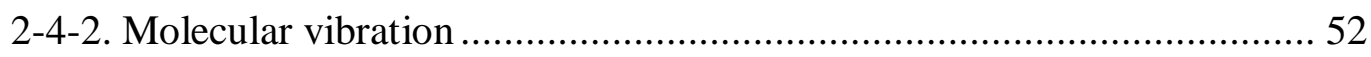

2-4-3. FTIR spectrum ........................................................................... 54 
2-5. Nuclear Magnetic Resonance (NMR) Spectroscopy.................................. 55

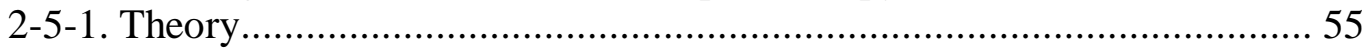

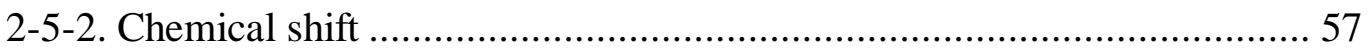

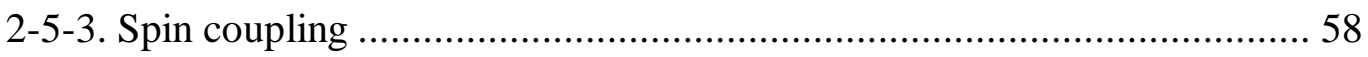

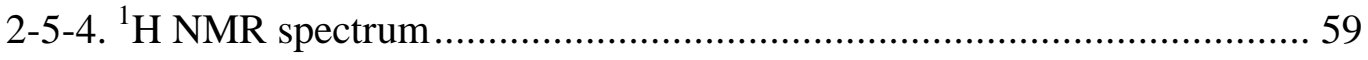

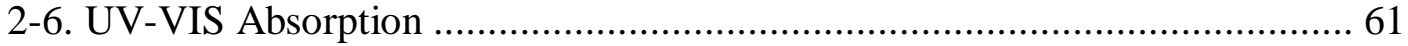

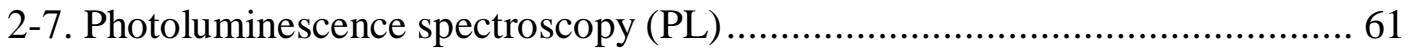

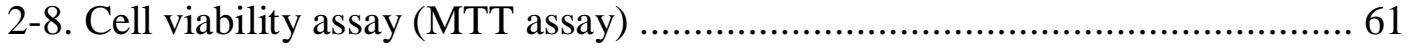

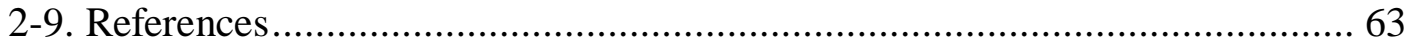

Chapter 3 Synthesis and surface modification of silicon quantum dots..............65

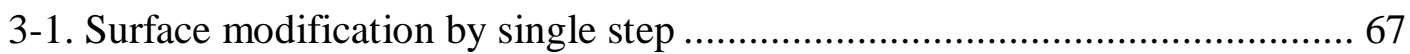

3-1-1. Silicon quantum dots capped with allylalcohol: Experiment 1 ................67 67

3-1-2. Silicon quantum dot capped with 1-hexene: Experiment 2, 3 ................ 68

3-1-3. Silicon quantum dot capped with 1,9-decadiene: Experiment 4.............. 75

3-1-4. Silicon quantum dot capped with 1,5-hexadiene ................................... 80

3-1-5. Silicon quantum dots capped with allylamine .................................... 89

3-1-6. Silicon quantum dots capped with methyl 10-undecenoate: Experiment 9

3-1-7. Silicon quantum dots capped with limonene: Experiment 10 ................100

3-1-8. Silicon quantum dots capped with propargylamine: Experiment 11 ......105

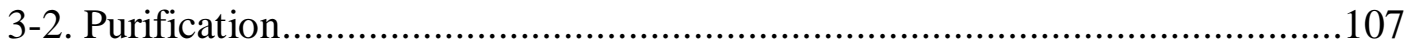

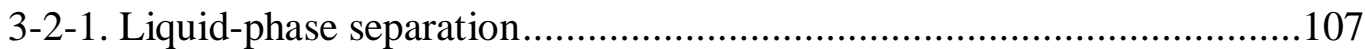

3-2-2. Size exclusion column chromatography............................................111

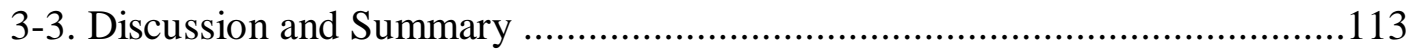

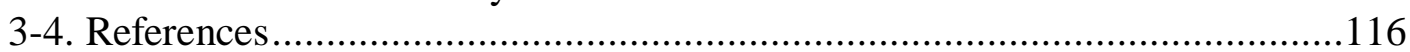

Chapter 4 Chemical reactions on the surface of silicon quantum dots and their biological application.....................................................................................................121

4-1. Chemical reactions of the surface moieties of the silicon quantum dots........123 Experiment 4-1-1: Epoxidation of terminal double bond of silicon quantum dots

Experiment 4-1-2: Oxidation of terminal epoxy ring of silicon quantum dots..127 Experiment 4-1-3: Bromination of terminal double bond of silicon quantum dots

Experiment 4-1-4: Conversion of the terminal double bond of silicon quantum dots to an alcohol functional group 
Experiment 4-1-5: Oxidation of terminal alcohol of silicon quantum dots to carboxylic acid

Experiment 4-1-6: Biotinylation of terminal amine group of silicon quantum dots

4-2. Optical properties of silicon quantum dots ............................................151

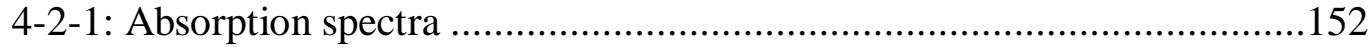

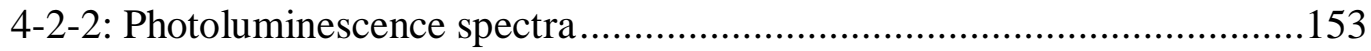

4-3. Cyto-toxicity evaluation of silicon quantum dots ......................................156

4-4. Bio-imaging of silicon quantum dots in cells ..........................................161

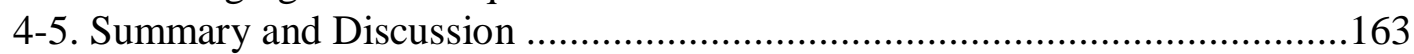

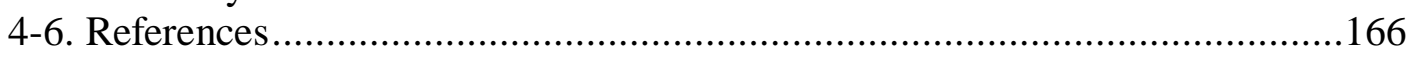

\section{Chapter 5 Microemulsion and high temperature bench top syntheses of} germanium quantum dots.................................................................................171

5-1. Microemulsion synthesis of germanium quantum dots..............................173

5-1-1. Syntheses of germanium quantum dots capped with allylamine ............174

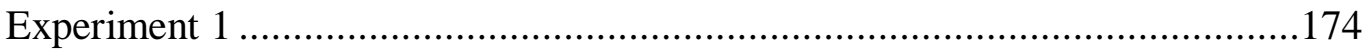

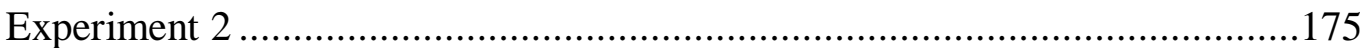

5-1-2. Syntheses of germanium quantum dots capped with 1,5-hexadiene.......178

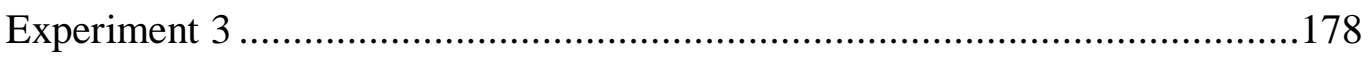

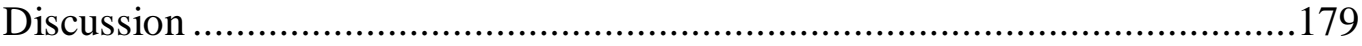

5-2. High temperature synthesis of germanium quantum dot.............................180

5-2-1. Reaction of triphenylgermanium chloride and sulfur in HDA

(hexadecylamine) with oleic acid capping at $260{ }^{\circ} \mathrm{C}$....................................181

Experiment 4

5-2-2. Reaction of triphenylgermanium chloride and sulfur in Oleylamine with mercaptoundecanoic acid capping at $290{ }^{\circ} \mathrm{C}$..............................................182

Experiment 5

5-2-3. Reaction of triphenylgermanium chloride and sulfur in Oleylamine with

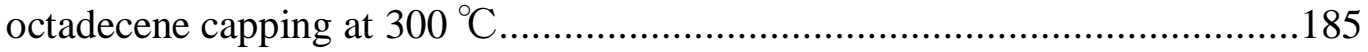

Experiment 6 .

5-2-4. Reaction of triphenylgermanium chloride and sulfur in Oleylamine with

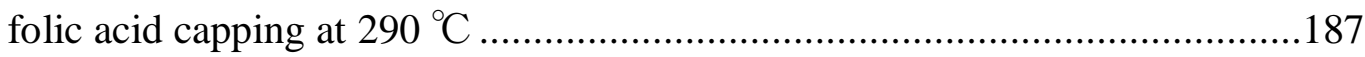

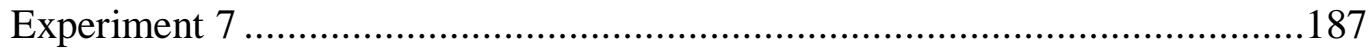

5-2-5. Reaction of triphenylgermanium chloride and sulfur in Trioctylamine with

11-dodecen 1-ol capping at $300{ }^{\circ} \mathrm{C}$ 
Experiment 8 .

5-2-6. Reaction of triphenylgermanium chloride and sulfur in Oleylamine with methyl 10 -undecenoate capping at $290{ }^{\circ} \mathrm{C}$..................................................193

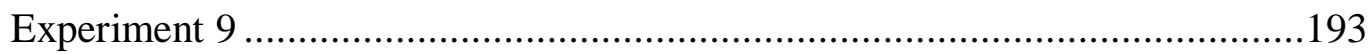

5-2-7. Reaction of triphenylgermanium chloride and sulfur in TOA (trioctyl

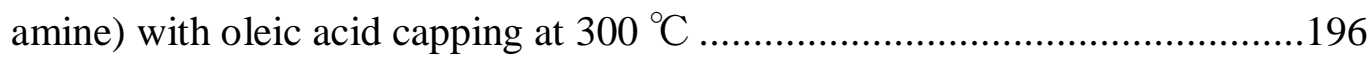

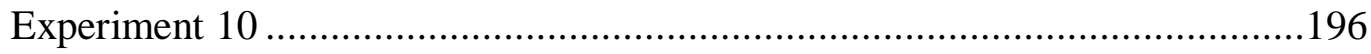

Conclusion and discussion of high temperature bench top syntheses ...............200

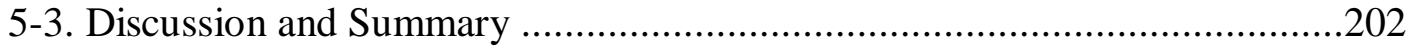

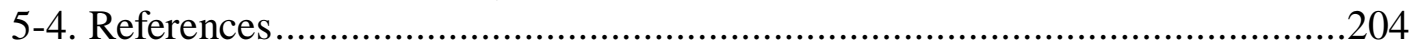

Chapter 6 Synthesis and Characterisation of Silica nanoparticles for dry eye diagnosis................................................................................................................208

6-1. Synthesis of organic dye doped silica nanoparticles .................................209

6-1-1. Rhodamine doped silica nanoparticles (Experiment 1 and 2) ...............209

6-1-2. Fluorescein doped silica nanoparticles (Experiment 3, 4 and 5) ...........214

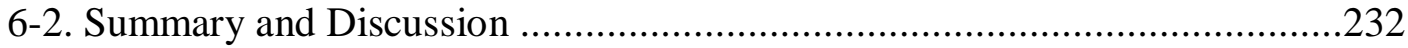

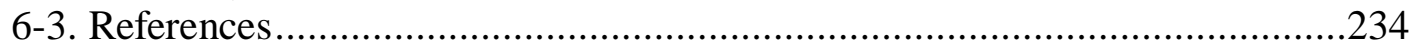

Chapter 7 Overall conclusions and future work ...............................................238

7-1. Synthesis and surface modification of silicon quantum dots......................238

$7-2$. Chemical reactions on the surface of silicon quantum dots and its biological

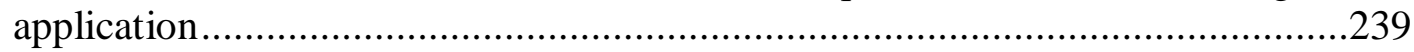

7-3. Syntheses of germanium quantum dots ................................................241

7-4. Synthesis and characterisation of silica nanoparticles for dry eye diagnosis .243

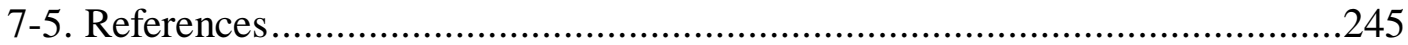




\section{Chapter 1 Introduction}

\section{1-1. Motivation and general overview of the research}

The research presented in this thesis is aimed at developing new semiconductor nanoparticles (quantum dots) for application into biological fields such as drug delivery systems and dry eye diagnosis.

Research on solution phase syntheses and surface modification of silicon quantum dots will be described in chapter 3 . In chapter 4 , chemical reactions on the surface of silicon quantum dots will be introduced for biological applications. In chapter 5, trials of high temperature bench top synthesis of germanium quantum dots will be described including the exploration of different capping agents. Chapter 6 describes the potential of silica nanoparticles into dry eye diagnosis applications.

Quantum dots have several interesting characteristics due to quantum effects that appear at the nanoscale. ${ }^{1,2}$ Because of these characteristics quantum dots have a range of possible applications such as lasers, photo sensors and are also being considered for use in medical biology. ${ }^{2-24}$ Current medical and biological fluorescent imaging is limited to the use of conventional organic dye markers, which are not photo stable. Quantum dots such as those of cadmium selenide, and silicon and germanium have been introduced as new fluorophores. As they have several advantages over conventional organic dyes ${ }^{3,5,25}$ Quantum dots possess unique optical and electronic properties which are not seen in their bulk counterparts. Quantum dots of groups II/VI such as cadmium selenide dots are well known and substantial research has been carried out on these systems ${ }^{26}$. Recently, quantum dots of group IV such as silicon or germanium dots are attracting more frequent attention. ${ }^{27-43}$

Quantum dots of group II/VI have strong luminescence compared to silicon and germanium quantum dots; however there have been concerns over the toxicity of these quantum dots in the human body. The toxicity of these quantum dots arises from 
two main sources; (1) the quantum dot core and (2) the capping molecules. The cytotoxicity of cadmium selenide quantum dots has been reported by Derfus et. al. ${ }^{44}$ who showed cell damage was caused by the cadmium selenide quantum dot core. Therefore, in comparison to cadmium selenide quantum dots silicon core nanoparticles would be an ideal candidate for biological fluorescence imaging. As with cadmium selenide quantum dots, silicon nanoparticles also offer the possibility to explore surface capping molecules which are hydrophilic and biocompatible.

Since silicon has well-known chemistry and the industrial benefits of being non-toxic and of low cost, interest in silicon nanoparticles has rapidly increased. Various different methods for the synthesis of silicon and germanium quantum dots have been reported. Solution phase synthesis of silicon nanoparticles using various reducing agents has been reported by Kauzlarich and co-workers. ${ }^{27-36}$ Silicon nanoparticles have also been synthesized by Korgel and co-workers in the condition of high temperature and high pressure ${ }^{37-41}$ and also with the aid of micelles by Wilcoxon et al. $^{42,43}$

This $\mathrm{PhD}$ project is focused on the synthesis of silicon quantum dots using microemulsion synthesis with a view towards biomedical applications. The syntheses included exploration of different purification methods and capping molecules to achieve the aim of application into the bio medical field. In addition, benchtop synthesis and microemulsion synthesis of germanium quantum dots were undertaken, and investigation of capping molecules carried out. Also synthesis of silica nanopaticles encapsulating organic dye towards the applications of dry eye diagnosis which targets to investigate tear film interactions was conducted. In the project, silicon nanoparticles are synthesized by the microemulsion synthesis technique which is carried out under oxygen free conditions to protect the quantum dot from oxidation using a strong reducing agent to achieve a narrow particle size distribution. In this reaction, chemicals such as tetraoctylammonium bromide (TOAB) and pentaethyleneglycol monododecyl ether $\left(\mathrm{C}_{12} \mathrm{E}_{5}\right)$ which are used as surfactants and also reducing agents such as lithium aluminium hydride $\left(\mathrm{LiAlH}_{4}\right)$ or lithium borohydride $\left(\mathrm{LiBH}_{4}\right)$ can be considered toxic to human body. Therefore investigation of various purification methods utilizing techniques such as column chromatography, liquid-liquid phase separation and precipitation to reduce the toxicity of those 
quantum dots to living cells or organisms will be carried out. In addition, surface molecules of quantum dots are very important to give them functionalities and it also affects their toxicity. On this topic, exploration of bio compatible capping molecules to attach to the surface will also be carried out.

\section{1-2. Quantum confinement effect}

The strongly size-dependent optical properties of semiconductor nanoparticles are well documented in the literature. ${ }^{45-47}$ This section aims to introduce succinctly the quantum confinement effect observed in semiconductor nanoparticles and nanowires. The band gap of a semiconductor can be viewed as the energy gap between the highest level of the valence band and the lowest level of the conduction band. After an electron is promoted to the conduction band by energy from photon, a hole is created in the valence band. This electron hole pair is regarded as an exciton. If the electron and hole form a bound state, the electron orbits the hole at a certain distance as in Bohr's model of the hydrogen atom. This distance, termed Bohr radius, is material dependent. For examples, the Bohr radius is $\sim 5 \mathrm{~nm}$ for silicon and $\sim 24.3 \mathrm{~nm}$ for germanium. When the physical size of a semiconductor particle is reduced to be smaller than its Bohr radius, the exciton becomes spatially confined and the confinement results in an increase in the band gap (a blue shift of the band gap). In general, the blue shift in the band gap becomes significant when the particle size is reduced to between 1 to $10 \mathrm{~nm}$. Semiconductor particles of sizes $<10 \mathrm{~nm}$ are often called quantum dots and therefore the effect of spatially confining an exciton in a semiconductor nanoparticle is termed the quantum confinement effect. A diagram for the quantum confinement effect is shown in Figure 1-1. 


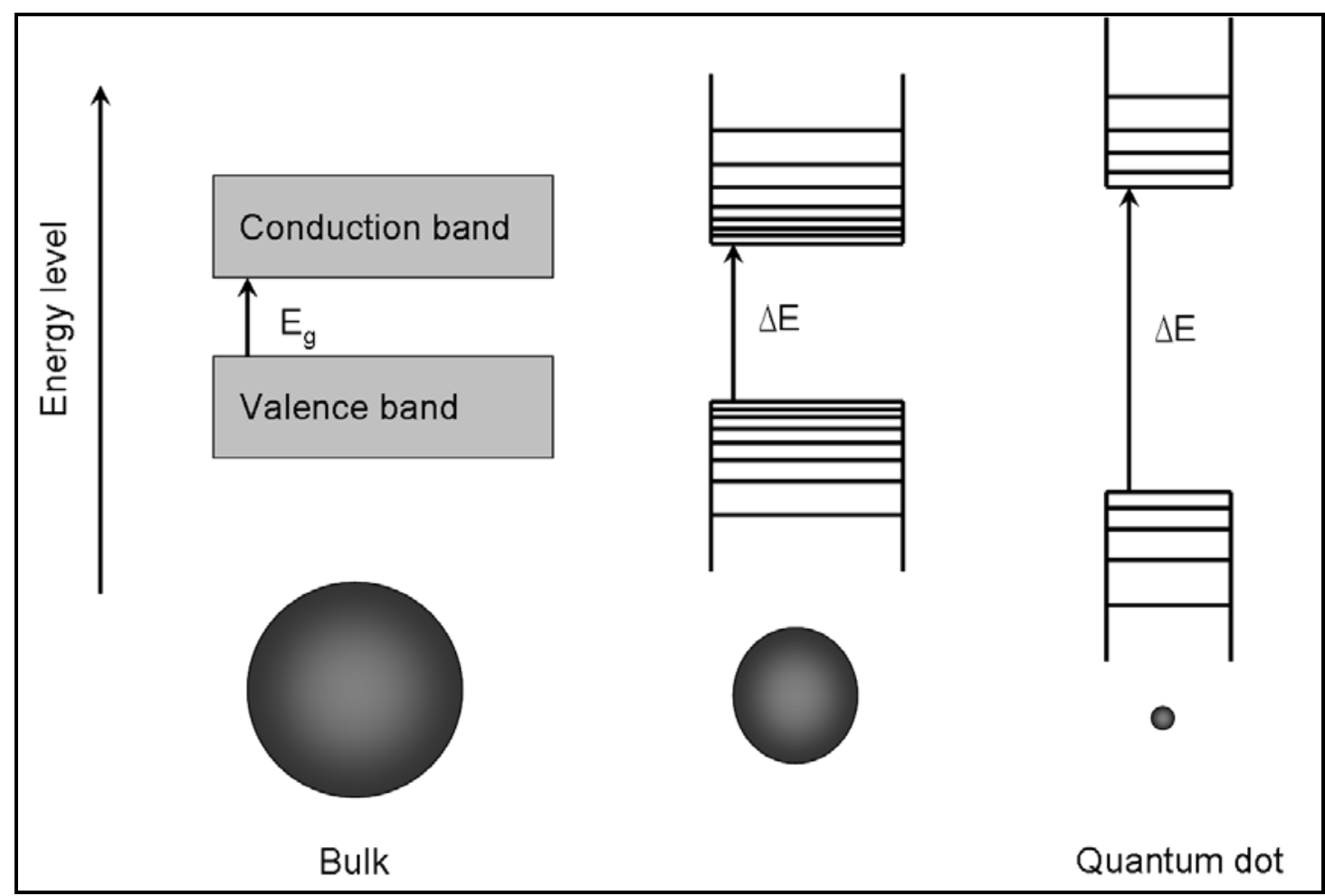

Figure 1-1: A diagram of the quantum confinement effect. Eg; energy difference between valence band and conduction band of bulk semiconductor material, $\Delta \mathrm{E}$; of size decreased semiconductor material

\section{1-3. Quantum dots}

Quantum dots (QDs) are semiconductor nano-materials in which the motion of excitons is restricted in all three directions. Quantum dots can be composed of elements in the periodic groups of II-VI, III-V, or IV-VI materials among others. Because of their small size, their energy values are quantized and their resulting behaviour is thus very different from their bulk counterparts. One of particular importance is their novel optical properties such as:

(1) The emission of quantum dots depends on their size.

(2) Because of their broad excitation spectra, different sized quantum dots can be excited at a single wavelength.

(3) They have excellent photo-stability and their fluorescence can be observed over longer time periods compared to organic dyes.

The aim of this project is to exploit those optical properties of quantum dots in biological fields by improving purification methods and surface modification. 


\section{1-3-1. Silicon quantum dots}

There are two types of semiconductors; direct bandgap and indirect bandgap materials. In direct bandgap semiconductors, the minimum energy level of the conduction band lies above the maximum energy level of the valence band at the same $\mathrm{k}$ vector. Therefore when the electron comes down to the valence band, momentum can be conserved which means electrons give off just a photon. On the other hand, in indirect bandgap semiconductors, the minimum energy level of the conduction band does not lie above the maximum energy level of valence band at the same $\mathrm{k}$ vector. Therefore when electrons recombine, there is a loss of momentum conservation due to the emission of a phonon but still a photon of lower energy emitted. In Figure 1-2, the energy band diagrams of silicon and gallium arsenide are shown. In Figure 1-2, $\mathrm{k}$ is the wave vector that represents angular momentum of electrons and specifies the wavenumber and the direction of propagation for a wave and $E$ is energy. In gallium arsenide as a direct bandgap semiconductor, the minimum energy level of conduction band lies above the maximum energy level of valence band. The momentum is defined by mass times velocity and photon has almost the zero mass. Therefore it is more likely to emit photon when electrons come down to valence band since the crystal momentum is conserved. On the other hand, in bulk silicon as an indirect bandgap semiconductor the minimum energy level of conduction band does not lie above the maximum energy level of valence band at the same k vector therefore, it is more likely to emit phonon rather than photon since phonon has momentum. The only optical transitions allowed are those where phonons are absorbed or emitted to conserve the crystal momentum. Therefore nanocrystalline silicon dots with a core size smaller than the exciton Bohr radius of silicon $(4.9 \mathrm{~nm})$ are expected to emit fluorescence. The emission of silicon quantum dots is considered to be based on competition between direct and quasi-direct recombination. ${ }^{48}$

Silicon quantum dots are spherical high surface area semiconductor particles. Nanoscale silicon particles are typically a few nanometres in size which leads to a large surface area to volume ratio. Silicon quantum dots have found application in luminescent display devices due to their broad excitation spectra and as building blocks for single-nanoparticle electronic devices, in micro and integrated semiconductors, and in solar energy cells. ${ }^{49,50}$ Further research is also looking into 
their potential electrical, optical, imaging, and bioscience applications. ${ }^{51}$ Basic properties of silicon quantum dot are shown in Table $1-1 .^{52}$

The crystal structure of silicon is based on the face centred cubic (FCC) lattice with additional atoms placed in the tetrahedral holes. This is the same structure as diamond. The structure is shown in Figure 1-3. This structure has silicon atoms at each corner of a unit cell and one atom on the centre of each face. In addition, there are four atoms lying within the cube. The lines between the silicon atoms in the lattice illustration indicate nearest-neighbour bonds. The unit cell dimension for the silicon is $0.543 \mathrm{~nm}$.

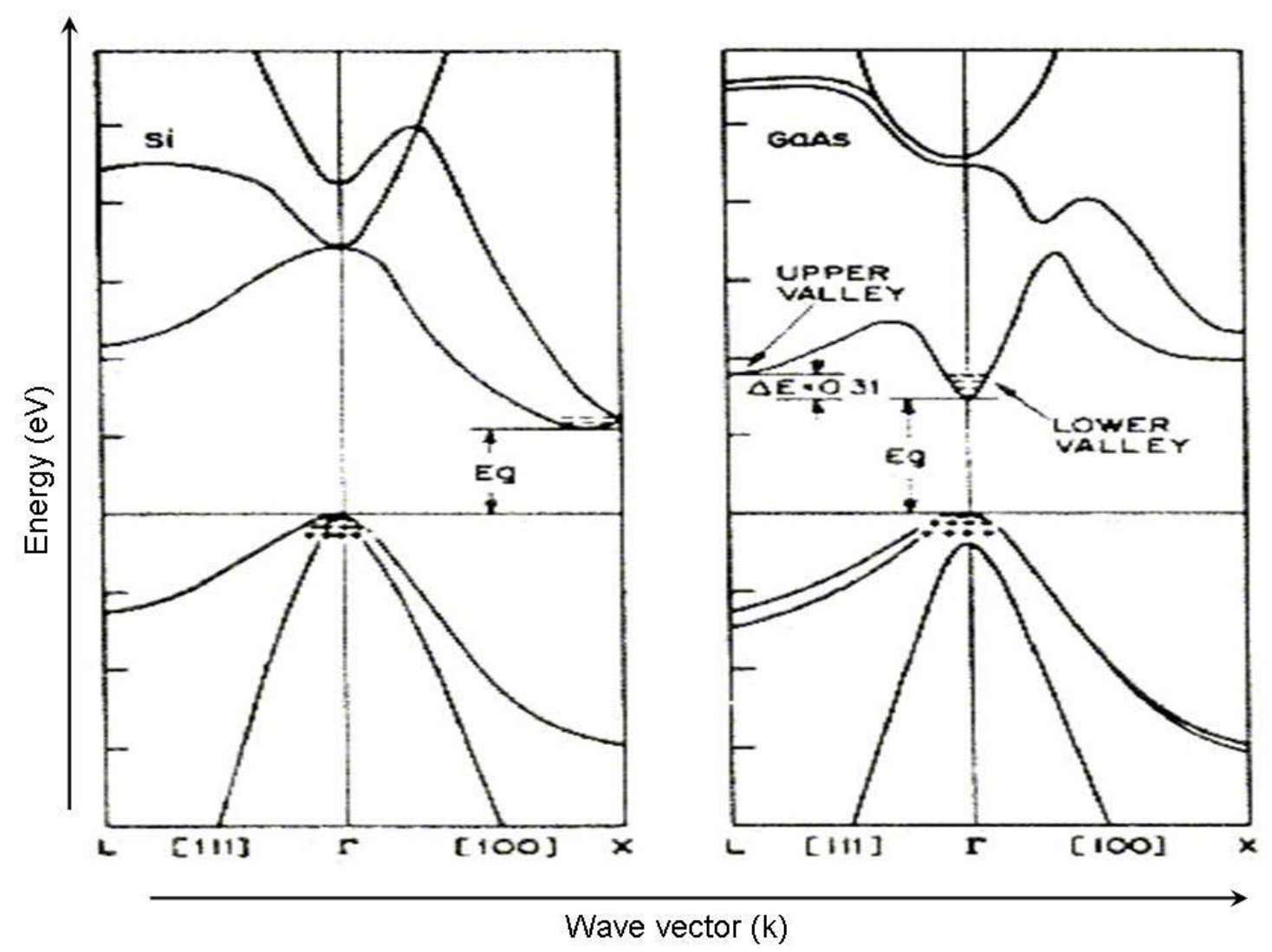

Figure 1-2: Energy bands diagrams of silicon and gallium arsenide 
Table 1-1: Basic properties of silicon quantum dots

\begin{tabular}{|l|l|}
\hline Structure & Diamond (FCC) \\
\hline Lattice parameter at $300 \mathrm{~K}$ & $0.54311 \mathrm{~nm}$ \\
\hline Band gap & Indirect \\
\hline Energy of Indirect band gap at $300 \mathrm{~K}$ & $1.12 \mathrm{eV}(1108 \mathrm{~nm})$ \\
\hline Energy of Direct band gap at 300 K & $3.4 \mathrm{eV}(366 \mathrm{~nm})$ \\
\hline Exciton Bohr Radius & $4.0 \mathrm{~nm}$ \\
\hline $\begin{array}{l}\text { Expected Range of Emission of } \\
\text { Quantum dots (<5 nm) }\end{array}$ & Visible \\
\hline
\end{tabular}

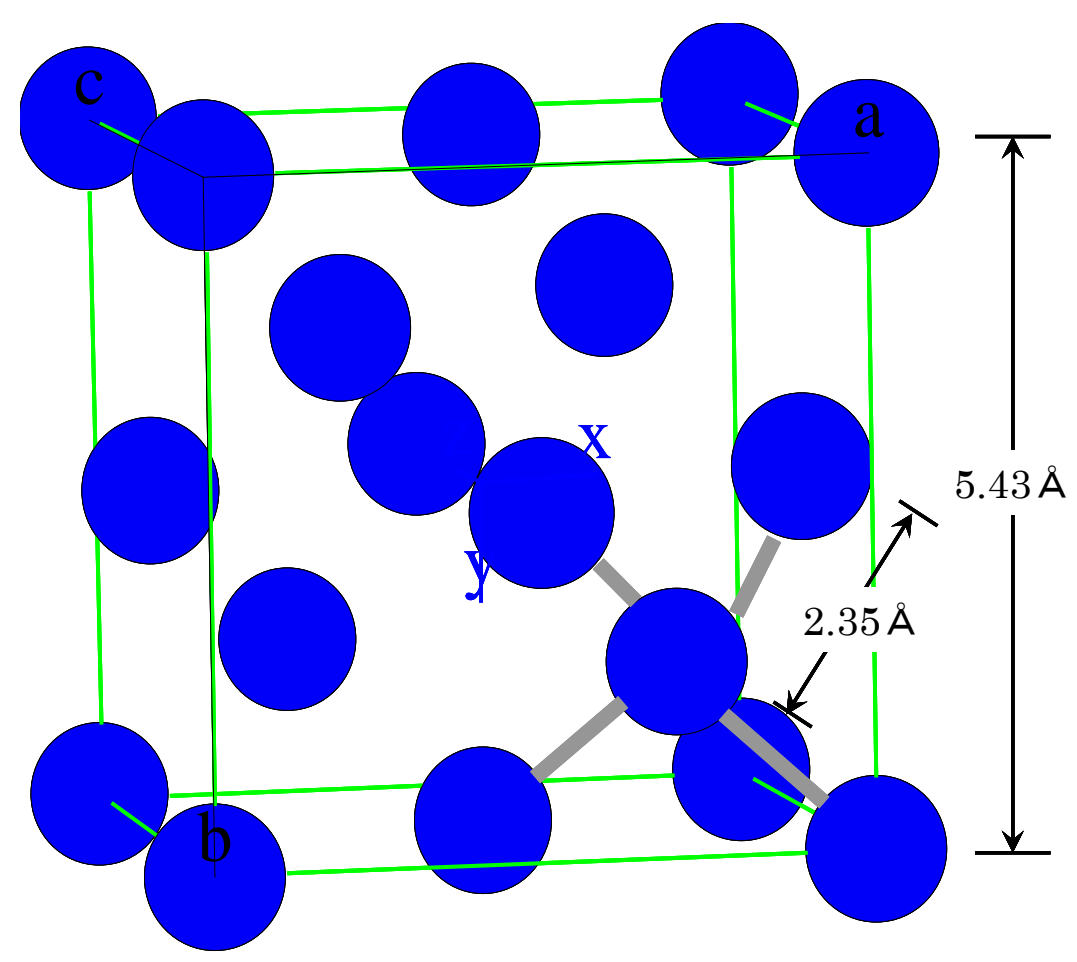

Figure 1-3: Crystal structure of silicon 


\section{1-3-2. Germanium quantum dots}

Although in many respects germanium and silicon systems are similar, germanium is characterized by a larger dielectric constant and smaller carrier masses, implying that quantum effects will be observable in relatively large germanium particles. ${ }^{53-57}$ Takagahara and Takeda have calculated the exciton confinement energy for finite sized silicon and germanium particles. ${ }^{58}$ They report confinement energy of $75 \AA$ germanium particles to be $0.5 \mathrm{eV}$. A similar magnitude effect is not observed in a silicon system until the particles are below $45 \AA$. Therefore fluorescent germanium quantum dots can be obtained with much larger sizes compared to silicon quantum dots. Germanium crystal structure is also the same as diamond.

Table 1-2: Basic properties of germanium quantum dots

\begin{tabular}{|l|l|}
\hline Structure & Diamond (FCC) \\
\hline Lattice parameter at $300 \mathrm{~K}$ & $0.565791 \mathrm{~nm}$ \\
\hline Band gap & Indirect \\
\hline $\begin{array}{l}\text { Energy of Indirect band gap at } \\
300 \mathrm{~K}\end{array}$ & $0.66 \mathrm{eV}(2000 \mathrm{~nm})$ \\
\hline $\begin{array}{l}\text { Energy of Direct band gap at } \\
300 \mathrm{~K}\end{array}$ & $0.8 \mathrm{eV}(1650 \mathrm{~nm})$ \\
\hline $\begin{array}{l}\text { Exciton Bohr Radius } \\
\text { Expected Range of Emission of } \\
\text { Quantum dots }(<5 \mathrm{~nm})\end{array}$ & Visible \\
\hline
\end{tabular}




\section{1-4. Representative syntheses of silicon quantum dots}

Solution phase synthesis is one of the most widely used methods to prepare silicon quantum dots. In this method, single crystals are grown from a liquid phase containing the precursor. There are multiple synthetic approaches including solvothermal synthesis as applied by Korgel et. $\mathrm{al}^{59}$ and microemulsion synthesis $^{42,43,60-62}$. In this project, microemulsion synthesis will be mainly used to make silicon quantum dots.

\section{1-4-1. Solvothermal synthesis}

In this synthesis, quantum dots are made under high pressure and high temperature conditions using solvents. Water is widely used as a solvent although organic solvents have been also investigated. There are several advantages of using a solvent with a high coordinating ability. These include formation of highly crystalline nanoparticles, highly monodisperse crystals due to the solvent molecules coordinating to the surface of the nanocrystals, preventing their aggregation, and increasing the luminous efficiency because of passivation of the particle surface by coordinated solvent molecules.

Silicon quantum dots synthesized by solvothermal synthesis have been reported by Heath in $1992 .^{63}$ This synthesis is based on reduction of silicontetrachloride and chloroalkylsilanes by sodium metal requiring high temperature and high pressure $\left(385^{\circ} \mathrm{C} />100 \mathrm{~atm}\right)$. The equation is shown below. The $\mathrm{R}$ group in these synthesis served as a surface-capping agent for the crystals. Therefore the ratio of the $\mathrm{RSiCl}_{3}$ to the $\mathrm{SiCl}_{4}$ reagent should be a measure of the surface-to volume ratio of the final crystallites.

$\mathrm{SiCl}_{4}+\mathrm{RSiCl}_{3}+7 \mathrm{Na} \longrightarrow 2 \mathrm{Si}$ (diamond lattice) $+7 \mathrm{NaCl}$

In this reaction, $\mathrm{R}=\mathrm{H}$, or octyl, and this $\mathrm{R}$ group serves as a surface capping agent for the crystal. 
Korgel and co-workers have reported the synthesis of alkyl terminated silicon quantum dots under supercritical conditions $\left(400-500{ }^{\circ} \mathrm{C} / 80 \mathrm{~atm}\right) .{ }^{39}$ These conditions resulted in particles with a diameter of between $0.15-0.4 \mathrm{~nm}$ which corresponds to a colour range from blue to green. On the other hand, alkyl-terminated silicon quantum dot have been synthesized at room reaction temperatures and at ambient pressure by Kauzlarich and co-workers. ${ }^{34}$ Zintl salts were used as the starting materials. This method has the potential to yield large numbers of silicon nanocrystals. The same synthetic method was also published using Grignard reagents as a capping agent. ${ }^{35}$

$\mathrm{Mg}_{2} \mathrm{Si}+\mathrm{SiCl}_{4} \longrightarrow \mathrm{MgCl}_{2}+\mathrm{Si}$ (chlorine terminated)

$\mathrm{Si}$ (chlorine terminated) $+\mathrm{RMgCl} \longmapsto \mathrm{Si}$ (alkyl terminated) $+\mathrm{MgCl}_{2}$

\section{1-4-2. Microemulsion Synthesis}

Compared to solvothermal synthesis, the reaction conditions of this method require neither high temperature nor high pressure. One of the most important advantages of this method is the ability to reduce the size distribution of the particles. Nanoparticles can be made easily by this method because growth of the nanoparticle is restricted by the reverse micelle. Usually in a typical micelle, surfactant in aqueous solution forms an aggregate with the hydrophilic "head" regions in contact with surrounding solvent, sequestering the hydrophobic tails regions in the micelle centre. However, in this synthesis the silicon quanutm dots were made in organic solvent therefore the surfactant forms an aggregate with the hydrophobic tails in contact with the solvent and sequetering the hydrophilic "head" in the micelle centre which is called reverse micelle. It is very important to produce highly monodispersed quantum dots for investigation of their optical properties. By this method, highly monodisperse nanocrystals can be produced at relatively low temperatures ${ }^{42,43,60-62}$. Surfactant molecules have both a hydrophobic and a hydrophilic end. By adding and homogenizing a small amount of water in an organic solvent with surfactant molecules, reverse micelles can be made. Nanoparticles are made by reducing the precursor within the reverse micelle. The surfactant molecules are then replaced with 
the desired capping species. Wilcoxon et al. reported reverse micelle silicon quantum dots synthesis. ${ }^{42,43}$ Halogenated silicon, $\mathrm{SiX}_{4}(\mathrm{X}=\mathrm{Cl}, \mathrm{Br}$, or I) was used as the precursor in the presence of surfactant such as pentaethylene glycol monododecyl ether $\left(\mathrm{C}_{12} \mathrm{E}_{5}\right)$. The silicon precursor is reduced by a reducing agent such as lithium aluminium hydride $\left(\mathrm{LiAlH}_{4}\right)$ to produce hydrogen terminated silicon quantum dots. The size distribution of the particles reported was between $2-10 \mathrm{~nm} .{ }^{62}$ The reaction scheme of this synthesis is shown in the later section.

Table 1-3 shows the summary of various synthesis methods for silicon quantum dots.

Table 1-3: Representative syntheses of silicon quantum dots

\begin{tabular}{|c|c|c|c|c|c|}
\hline Precursor & $\begin{array}{l}\text { Reducing } \\
\text { agent }\end{array}$ & $\begin{array}{l}\text { Reaction } \\
\text { condition }\end{array}$ & $\begin{array}{l}\text { Solvent/ } \\
\text { Surfactant }\end{array}$ & $\begin{array}{l}\text { Particle } \\
\text { size }\end{array}$ & References \\
\hline $\begin{array}{l}\mathrm{SiCl}_{4}+ \\
\mathrm{RSiCl}_{3}\end{array}$ & $\mathrm{Na}$ in Toluene & $\begin{array}{l}>100 \text { atm } \\
\text { and } 385{ }^{\circ} \mathrm{C}\end{array}$ & $\begin{array}{l}\text { Hexane or } \\
\text { pentane }\end{array}$ & $\sim 300 \mathrm{~nm}$ & 63 \\
\hline$(\mathrm{Ph})_{2} \mathrm{SiH}_{2}$ & & $\begin{array}{l}80 \text { atm and } \\
400-500^{\circ} \mathrm{C}\end{array}$ & $\begin{array}{l}\text { 1-octanol } \\
\text { and } \\
\text { hexane }\end{array}$ & $\sim 4 \mathrm{~nm}$ & 39,40 \\
\hline $\mathrm{SiCl}_{4}$ & $\begin{array}{l}\text { Grignard } \\
\text { reagent }\end{array}$ & $\begin{array}{l}\text { Room } \\
\text { temperature }\end{array}$ & Glyme & $\sim 5 \mathrm{~nm}$ & $\begin{array}{l}28,29,32, \\
34\end{array}$ \\
\hline $\begin{array}{l}\mathrm{SiX}_{4} \\
\text { X=Cl, } \mathrm{Br} \\
\text { or I }\end{array}$ & $\begin{array}{l}\mathrm{LiAlH}_{4} \quad \text { in } \\
\text { THF }\end{array}$ & $\begin{array}{l}\text { Room } \\
\text { temperature } \\
\text { / under } \mathrm{N}_{2}\end{array}$ & $\begin{array}{l}\text { Octane or } \\
\text { decane }\end{array}$ & $\sim 2 \mathrm{~nm}$ & 42,43 \\
\hline $\begin{array}{l}\mathrm{SiX}_{4} \\
\text { X=Cl, Br } \\
\text { or I }\end{array}$ & $\begin{array}{l}\mathrm{LiAlH}_{4} \quad \text { or } \\
\text { superhydride }\end{array}$ & $\begin{array}{l}\text { Room } \\
\text { temperature } \\
\text { / under } \mathrm{N}_{2}\end{array}$ & $\begin{array}{l}\text { Toluene or } \\
\text { hexane }\end{array}$ & $\sim 2 \mathrm{~nm}$ & $60-62$ \\
\hline
\end{tabular}




\section{1-5. Representative syntheses of germanium quantum dots}

Since germanium has quite similar characteristics as silicon, germanium quantum dots are also attractive as a potential candidate for applications in biological fields. However, compared to the amount of research on silicon quantum dots, there has been relatively little work done on the synthesis of germanium quantum dots. There are various chemical methods including hydrolysis of germanium tetrachloride in Zeolite $\mathrm{Y}$ followed by $\mathrm{H}_{2}$ reduction, reduction of germanium tetrachloride and $\mathrm{RGeCl}_{3}$ by sodium dispersion in heptane and reduction of germanium tetrachloride by lithium naphthlide. $^{64}$

Heath and co workers reported the synthesis of germanium quantum dots 2-20 nm in size by the reduction of germanium tetrachloride by sodium-potassium alloy at $270{ }^{\circ} \mathrm{C} .{ }^{65,66}$

$\mathrm{GeCl}_{4}+\mathrm{RMCl}_{3}+\mathrm{RMCl}_{2}+\mathrm{NaK} \longrightarrow \mathrm{Ge}(\mathrm{R}$ terminated $)+\mathrm{NaCl}+\mathrm{KCl}$

M: germanium

Miguez $\mathrm{H}$ et al. reported a method for obtaining germanium nanocrystals using the porous structure of zeolite $\mathrm{Y}$ as a matrix. Samples were obtained by reduction of germanium dioxide nanocrystals, previously grown in zeolite alpha cages, in $\mathrm{H}_{2}$ at $470{ }^{\circ} \mathrm{C} .{ }^{67}$

$\mathrm{GeCl}_{4}+2 \mathrm{H}_{2} \mathrm{O} \longrightarrow \mathrm{GeO}_{2}+4 \mathrm{HCl}$

$\mathrm{GeO}_{2}+2 \mathrm{H}_{2} \longrightarrow \mathrm{Ge}+2 \mathrm{H}_{2} \mathrm{O}$

However these syntheses have drawbacks. The reduction of germanium tetrachloride and $\mathrm{RGeCl}_{3}$ by sodium-potassium alloy dispersion in heptane requires high temperature and high pressure to force crystallization and neither method permits control over particle size or surface termination. Kauzlarich and co-workers 
synthesized alkyl terminated germanium quantum dots by the reduction of germanium tetrachloride by Zintl salts such as sodium-germanium/potassiumgermanium/magnesium-germanium ( $\mathrm{NaGe} / \mathrm{KGe} / \mathrm{Mg}_{2} \mathrm{Ge}$ ) in Glyme. The particle size distribution obtained in this synthesis is very narrow and the mean diameter of the germanium particles was $6.2-6.5 \mathrm{~nm}^{68-70}$

$\mathrm{Mg}_{2} \mathrm{Ge}+\mathrm{GeCl}_{4} \longrightarrow \mathrm{MgCl}_{2}+\mathrm{Ge}$ (chlorine terminated)

$\mathrm{Ge}$ (chlorine terminated) $+\mathrm{RMgCl} \longrightarrow \mathrm{Ge}$ (alkyl terminated) $+\mathrm{MgCl}_{2}$

Warner et al. have also synthesised germanium quantum dots by reducing germanium tetrachloride with lithium trimethyl borohydride (superhydride) in an inverse micelle solution at room temperature. Using this method, different sized water soluble photoluminescent germanium quantum dots ( $5 \mathrm{~nm}$ and $50 \mathrm{~nm}$ ) have been synthesized by varying the speed of addition of the reducing agent. Allylamine was used as a capping agent to make the particles hydrophilic. ${ }^{71,72}$ A brief summary of germanium quantum dot synthesis are shown in Table 1-4.

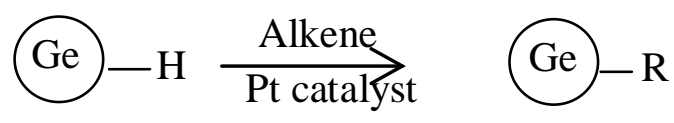


Table 1-4: Liquid phase of germanium quantum dots

\begin{tabular}{|c|c|c|c|c|c|}
\hline Precursor & $\begin{array}{l}\text { Reducing } \\
\text { agent }\end{array}$ & $\begin{array}{l}\text { Reaction } \\
\text { condition }\end{array}$ & $\begin{array}{l}\text { Solvent/ } \\
\text { Surfactant }\end{array}$ & $\begin{array}{l}\text { Particle } \\
\text { size }\end{array}$ & References \\
\hline $\begin{array}{l}\mathrm{GeCl}_{4}+ \\
\text { Zintl salt }\end{array}$ & $\mathrm{NaK}$ & $\begin{array}{l}270{ }^{\circ} \mathrm{C} \text { and } \\
\text { high pressure } \\
\text { (not specific } \\
\text { value } \\
\text { given) is }\end{array}$ & Glyme & $\sim 11 \mathrm{~nm}$ & 65,66 \\
\hline $\begin{array}{l}\mathrm{GeCl}_{4}+ \\
\mathrm{GeO}_{2}\end{array}$ & $\mathrm{H}_{2}$ & $470{ }^{\circ} \mathrm{C}$ & & $\sim 3 \mathrm{~nm}$ & 67 \\
\hline $\mathrm{GeCl}_{4}$ & $\begin{array}{l}\mathrm{NaGe} / \\
\mathrm{KGe} / \\
\mathrm{Mg}_{2} \mathrm{Ge}\end{array}$ & & Glyme & $\begin{array}{l}6.2^{-6.5} \\
\mathrm{~nm}\end{array}$ & 54,64 \\
\hline $\mathrm{GeCl}_{4}$ & $\mathrm{LiAlH}_{4}$ & $\begin{array}{l}\text { Room } \\
\text { temperature/ } \\
\text { under } \mathrm{N}_{2}\end{array}$ & $\begin{array}{l}\text { Toluene/ } \\
\text { TOAB }\end{array}$ & $\sim 5 \mathrm{~nm}$ & 71,72 \\
\hline $\mathrm{Ph}_{3} \mathrm{GeCl}$ & & $280 \sim 300{ }^{\circ} \mathrm{C}$ & $\begin{array}{l}\text { TOA or } \\
\text { HDA } \\
\text { / } \\
\text { Oleylamine }\end{array}$ & $\sim 8 \mathrm{~nm}$ & $\begin{array}{l}\text { Tilley et } \\
\text { al. } \\
\text { (not } \\
\text { published) }\end{array}$ \\
\hline
\end{tabular}

\section{1-6. Capping technique of quantum dots for biological applications}

To use quantum dots in various applications, specific surface modification should be conducted for each purpose. For biological applications in particular, water soluble, non-toxic quantum dots are required.

The quantum dots synthesised initially were not biocompatible because of the nonpolar organic functional groups exposed on their surface. ${ }^{73,74}$ However Chan et al. solved this problem by synthesising cadmium-selenide/zinc-sulfur (CdSe/ZnS) core shell quantum dots coated with mercaptoacetic acid and covalently coupled to a protein. ${ }^{75}$ This report opened the door to a wide array of possible applications and 
many hydrophilic capping techniques have since been reported. Gerion et al have reported silica coated and MUA (mercapto undecanoic acid) coated CdSe/ZnS core shell quantum dots ${ }^{65}$. One of the difficulties in surface modification of quantum dots for biological applications is that they can easily aggregate under biological conditions because they are unstable under either acidic or saline conditions ${ }^{76}$.

For biological applications, it is necessary to make quantum dots stable under acidic or saline conditions. Quantum dots conjugated with biomolecules are difficult to produce because most of the biomolecules exist in the isotonic condition in vivo. ${ }^{77,78}$ There is a limited number of these molecular species that can conjugate with quantum dots. Some molecules lose their functionality when they are attached to the surface of quantum dots or some molecules do not have the appropriate reactive part to bind to the surface of the quantum dots. Silica coated cadmium-selenide quantum dots that show high stability under acidic conditions have been reported by Gerion et al. ${ }^{76}$ In order to apply these quantum dots into biological fields, biocompatible capping molecules have been investigated as well. Hwang et al. reported on a dendric cellulose coating method for cadmium-sulfur(CdS) quantum dots. ${ }^{79}$ The cadmiumsulfur/cellulose hybrid was prepared by alternating drop-wise addition of cadmium ions and sulfide ions to a solution containing $\left\{\left[\left(\mathrm{HO}_{2} \mathrm{C}\right)_{27}-\mathrm{Den}\right]\right.$-cellulose $\}$. Cytotoxicity of this quantum dot was evaluated and the results showed that this dot has negligible cyto-toxicity due to dendron cohesion. In addition, polyethyleneglycol (PEG) coated cadmium-selenide quantum dots and techniques to disperse quantum dots in biological conditions by coating with serum albumin have been investigated by several groups. ${ }^{80}$ However, yet another problem arose. Since PEG is a large molecule, the size of the quantum dots increases when coated with PEG. This is not convenient for some biological applications such as bio imaging. Furthermore, there are some reports warning about toxicity of cadmium-selenide quantum dots because there is a risk of cadmium ion leak. Bhatia et al reported that when such dots are exposed to UV light, cadmium ion leaks from the dots and can cause serious cell damage. ${ }^{81}$ As a result, interest in the synthesis of biocompatible silicon quantum dots has increased because silicon is not a heavy metal, unlike cadmium, and bulk silicon material is safe for organs. Since silicon quantum dots are able to be covalently bound to carbon or oxygen containing molecules there are a few reports on silicon quantum dots capped with hydrophobic molecules such as 1-hexene and 1-heptene. ${ }^{60}$ 
Preparation of water-dispersible silicon quantum dots that maintain their stability of photoluminescence has proven difficult. Acrylic acid (PAAc) coated silicon quantum dots have been reported by Ruckenstein et al. ${ }^{82}$ These silicon quantum dots are quite stable in aqueous solutions with good photoluminescent stability. Swihart et al. also reported propionic acid coated silicon quantum dots. In both cases, silicon dots were capped under UV light irradiation. ${ }^{83,84}$ Surface modification by UV irradiation as well as platinum catalyst will be described in later section.

Warner et. al. attached allylamine to silicon quantum dots using a different method to provide an amino-termination. These amino-terminated silicon quantum dots were made in an inverse micelle system and capped with allylamine using a platinum catalyst. ${ }^{51}$ However, there are still only a few reports describing the surface modification of silicon quantum dots. Therefore, there are still many possibilities to explore in this area. The investigation of capping molecules and capping techniques of silicon quantum dots for biological applications will be described in the chapter 3 and 4 . 
Table 1-5: Capping techniques of different quantum dots

\begin{tabular}{|l|l|l|l|}
\hline Particles & Capping molecules & $\begin{array}{l}\text { Capping } \\
\text { methods }\end{array}$ & References \\
\hline $\begin{array}{l}\text { CdSe/ZnS } \\
\text { core shell }\end{array}$ & $\begin{array}{l}\text { Mercaptoacetic acid / } \\
\text { protein }\end{array}$ & $\begin{array}{l}\text { Cleavage } \\
\text { reaction }\end{array}$ & 75 \\
\hline CdSe dot & Silane precursors & $\begin{array}{l}\text { Reverse } \\
\text { microemulsion }\end{array}$ & 76 \\
\hline CdS dot & $\left\{\left[\left(\mathrm{HO}_{2} \mathrm{C}\right)_{27}\right.\right.$-Den]-cellulose $\}$ & Stirring & 79 \\
\hline Si dot & $\begin{array}{l}\text { Acid } \\
\text { acid/Acrylic acid) }\end{array}$ & $\begin{array}{l}\text { (Propicnic } \\
\text { 1-hexane/ allylamine irradiation }\end{array}$ & $82-85$ \\
\hline Si dot & \multicolumn{2}{|c|}{$\begin{array}{l}\text { Catalytic } \\
\text { reaction }\end{array}$} & $60-62$ \\
\hline
\end{tabular}

\section{1-6-1. Mechanism of UV-irradiation capping}

To avoid the risk of forming platinum nanoparticles UV-irradiation capping using the white light, $254 \mathrm{~nm}$, can be considered as alternative option. There two possible mechanisms have been proposed for the light-promoted hydrosilylation reaction. ${ }^{86}$

The first mechanism being proposed involves homolysis of a surface $\mathrm{Si}-\mathrm{H}$ bond, forming a silicon radical. Since silicon radical are known to react very rapidly with unsatulated carbon-carbon bonds, Si-C bond forming is expected to be a facile step, forming the surface bond carbonbased radical on the $\beta$-carbon. Then this carbon based radical abstract a neighbouring hydrogen and complete the hydrosilylation. The reaction mechanism is shown in scheme 1-6-1. 


\section{UV light}
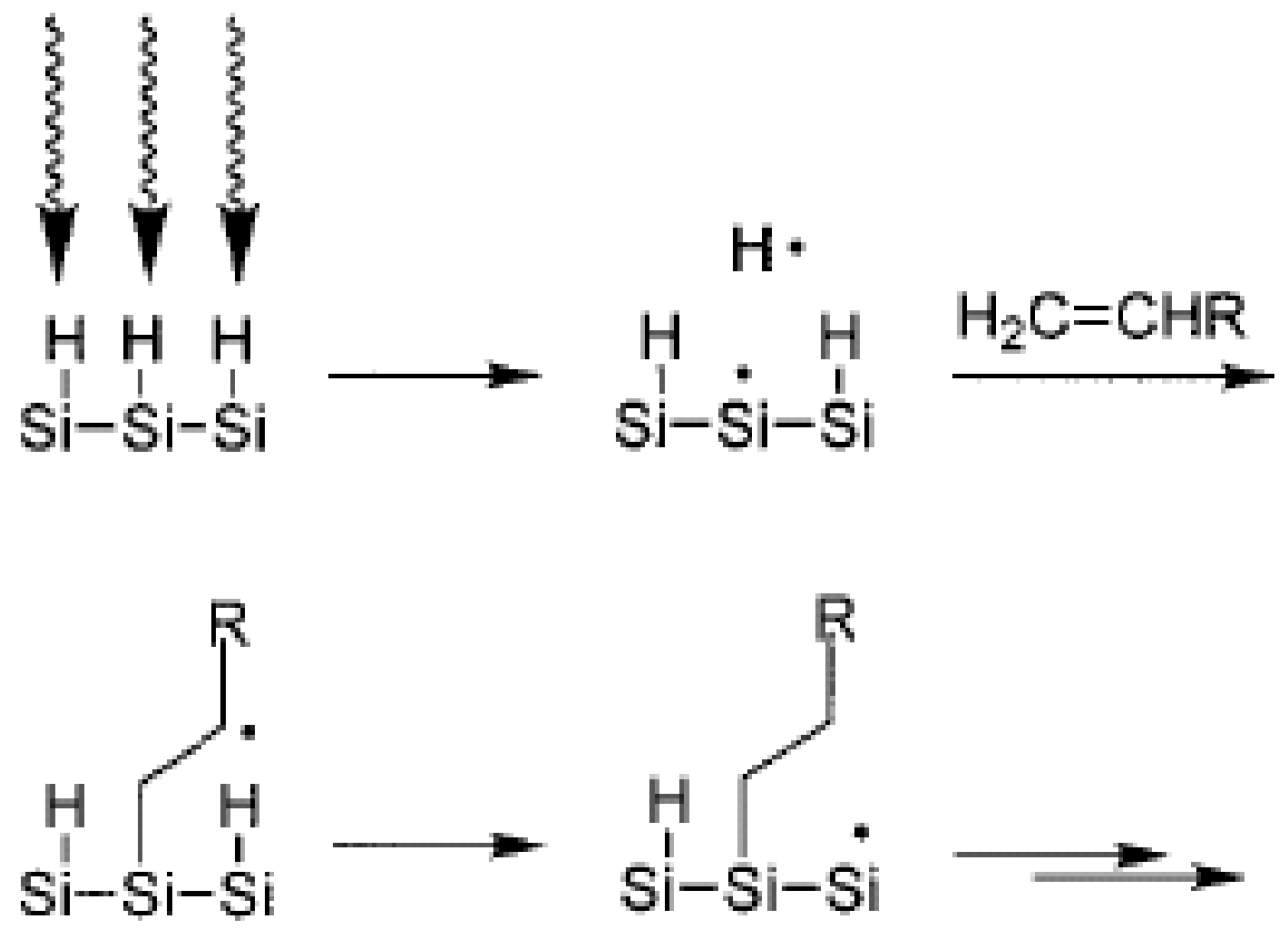

Scheme 1-6-1: Reaction mechanism of UV-light promoted hydrosilylation reaction by forming a silicon radical

The second mechamisn being proposed is opposed to Si-H homolysis. The excitons form reactive surface states that are a byproduct of room temperature photoluminescence. Scheme 1-6-2 shows the proposed mechanism for the UV-light promoted hydrosilylation reaction begins with the formation of a complex between an adsorbed alkene and the surface-localised hole. Attack by an alkene nucleophile at an electrophilic silicon centre is known in the chemistry of silyl cations in the gas phase. The reaction proceeds directly and irreversibly to form the Si-C bond, resulting in a carbocation stabilised by a $\beta$-silyl group. The high strength and low polarity of the nascent Si-C bond should limit the reversibility of this step. The strongly acidic carbocation can then abstract a hydride from an adjacent hydridic Si-H bond, forming a stronger and less polar $\mathrm{C}-\mathrm{H}$ bond. This hydride could be formally the product of a hydrogen atom and the electron half of the original exciton $\mathrm{e}-\mathrm{h}+$ pair. This hydride abstraction step by a $\beta$-silyl carbocation is proposed in mechanisms related to solution-phase hydrosilylation. The reaction mechanism is shown in scheme 1-6-2. 


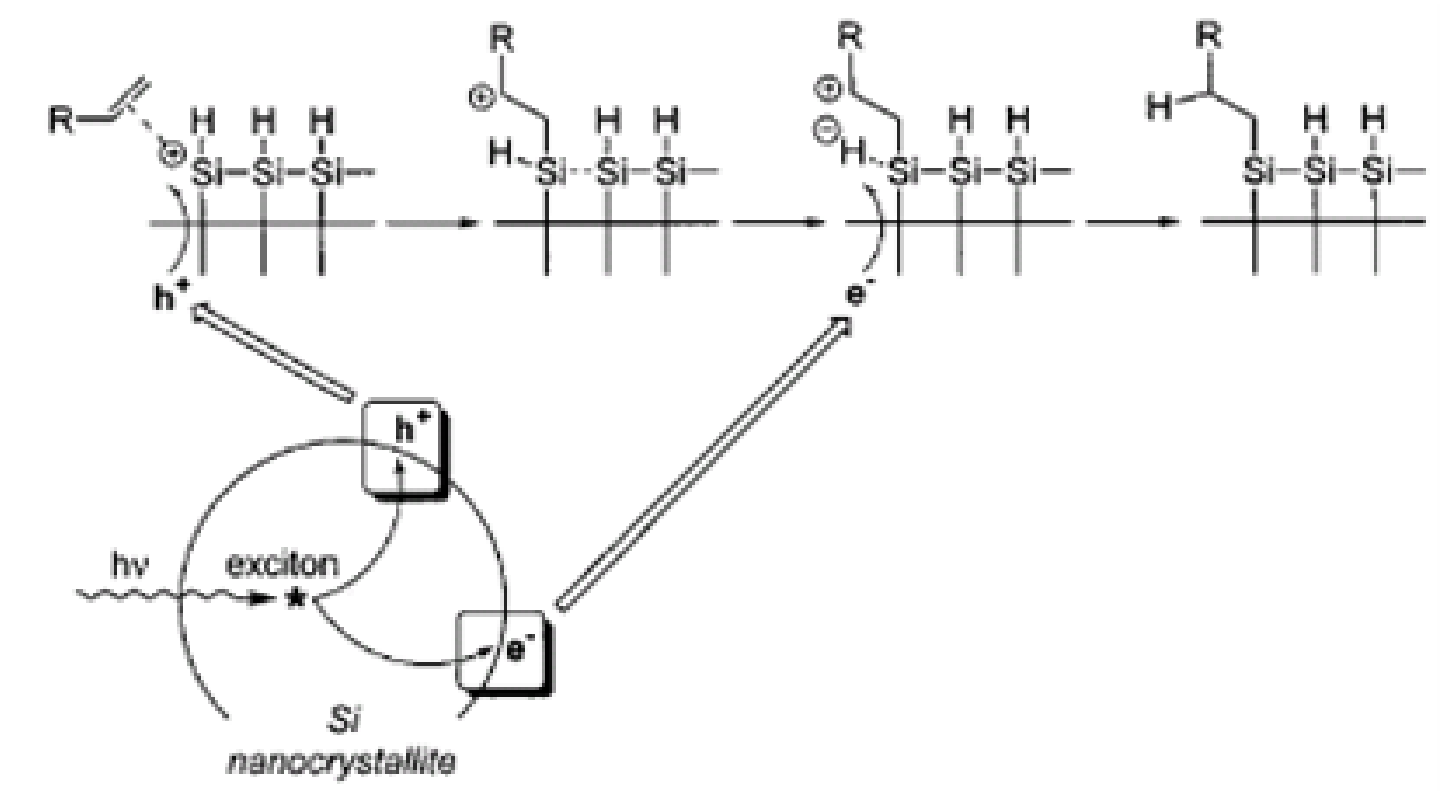

Scheme 1-6-2: Reaction mechanism of UV-light promoted hydrosilylation reaction by forming a carbocation

\section{1-7. Purification of silicon quantum dots}

Due to their low toxicity, silicon or germanium quantum dots have been considered as good potential candidates for a range of applications especially in medical biology. ${ }^{61}$, ${ }^{62}$ However, there are still more steps to be taken before effective application is possible. Purification is one of them. Utilisation of the microemulsion synthesis method requires several ingredients such as strong reducing agents, surfactants and capping agents, some of which are toxic to humans. As a result proper purification is essential. However, little attention has been afforded to this issue so far.

Typical purification methods include the use of column chromatography, precipitation, liquid-liquid phase separation and dialysis. Purification processes are also dependant on the surface molecules of the quantum dots. Colvin et al. reported on the purification of alkyl terminated cadmium selenide quantum dots using size exclusion chromatography. The advantage of this method is quantitative information of quantum dot size and size distribution can be obtained. A porous cross-linked, polystyrene column $(300 * 7.5 \mathrm{~mm})$ of $1000-\AA$ pore size and $5-\mu$ m particle size was used. ${ }^{87,88}$ Dialysis purification of gold, silver and platinum nanoparticles was reported by Hsing-Lin Wang and co-workers. ${ }^{89}$ The synthetic and purification procedure of 
those particles is carried out in an aqueous solution therefore it is environmentally benign and potentially suitable for large scale production. Buuren and Galli reported purification of germanium quantum dots by burning the impurities of germanium quantum dots at $600^{\circ} \mathrm{C} .{ }^{90}$ In this case, the quantum dots lose about $5 \%$ of their weight, however XRD analysis still shows an identical diffraction pattern. However this method has a risk of oxidation and may not be suitable for surface functionalisation. Our group has reported a method of purifying alkyl terminated silicon quantum dots by washing with $\mathrm{N}$-methylformamide using a liquid-liquid phase separation method. ${ }^{60,62}$ Since this separation method is based on the polarity difference, it is possible to separate the particles from surfactant that has experienced significant agglomeration. We have also reported the purification of hydrophilic terminated germanium quantum dots by extraction and filtration using polarity. ${ }^{71}$ A method for purifying hydrophobic terminated germanium quantum dots using a precipitation method has been reported by Korgel et al. ${ }^{91}$ However, this method requires the particles to be large enough to precipitate. 
Table 1-6: Basic purification methods for nanoparticles

\begin{tabular}{|c|c|c|c|c|}
\hline Particle & $\begin{array}{l}\text { Purification } \\
\text { method }\end{array}$ & Surface & Impurities & Reference \\
\hline $\begin{array}{l}\text { CdSe } \\
\text { quantum } \\
\text { dots }\end{array}$ & $\begin{array}{l}\text { Size } \\
\text { exclusion } \\
\text { column }\end{array}$ & $\begin{array}{l}\text { Alkyl } \\
\text { terminated }\end{array}$ & TOPO & 87,88 \\
\hline $\begin{array}{l}\text { Germanium } \\
\text { quantum } \\
\text { dots }\end{array}$ & Burning & & Hydrocarbons & 90 \\
\hline $\begin{array}{l}\text { Germanium } \\
\text { quantum } \\
\text { dots }\end{array}$ & Precipitation & $\begin{array}{l}\text { Alkyl } \\
\text { terminated }\end{array}$ & TOP/TBP & 91 \\
\hline $\begin{array}{l}\text { Germanium } \\
\text { quantum } \\
\text { dots }\end{array}$ & $\begin{array}{l}\text { Extraction } \\
\text { and filtration }\end{array}$ & $\begin{array}{l}\text { Allylamine } \\
\text { (hydrophilic) }\end{array}$ & $\begin{array}{l}\text { TOAB } \\
\text { (surfactant) }\end{array}$ & 71,72 \\
\hline $\begin{array}{l}\text { Silicon } \\
\text { quantum } \\
\text { dots }\end{array}$ & $\begin{array}{l}\text { Liquid-liquid } \\
\text { phase } \\
\text { separation }\end{array}$ & $\begin{array}{l}\text { Alkyl } \\
\text { terminated }\end{array}$ & $\begin{array}{l}\text { TOAB/C }{ }_{12} \mathrm{E}_{5} \\
\text { (surfactant) }\end{array}$ & 60,62 \\
\hline $\begin{array}{l}\mathrm{Au} \text { and } \mathrm{Ag} \\
\text { particles }\end{array}$ & Dialysis & $\begin{array}{l}\text { Biological } \\
\text { molecules }\end{array}$ & Salt tec. & 89 \\
\hline
\end{tabular}

\section{1-7-1. Mechanism of size exclusion chromatography}

Since free capping agents have the same polarity group as the silicon quantum dots capped with the agent therefore it is impossible to separate them by surface charge. Also surfactant molecules have both polar and non-polar groups in the molecule and it makes difficulties to use ion exchange choromatography. Therefore separating silicon quantum dots from other materials by size can be an alternative option. Size exclusion column chromatography separates molecules according to their size. Smaller molecules are able to enter the pores of the media and therefore the molecules are trapped and removed from the flow of the mobile phase. However the molecules that 
are larger than the average pore size of the packing materials are excluded and they are the first to be eluted. ${ }^{92}$ The mechanism of size exclusion column chromatography is shown in Figure 1-4. ${ }^{93} \mathrm{LH} 20$ was chosen as the packing material in this project. (http://www.chemistry.adelaide.edu.au/external/soc-rel/content/size-exc.htm)

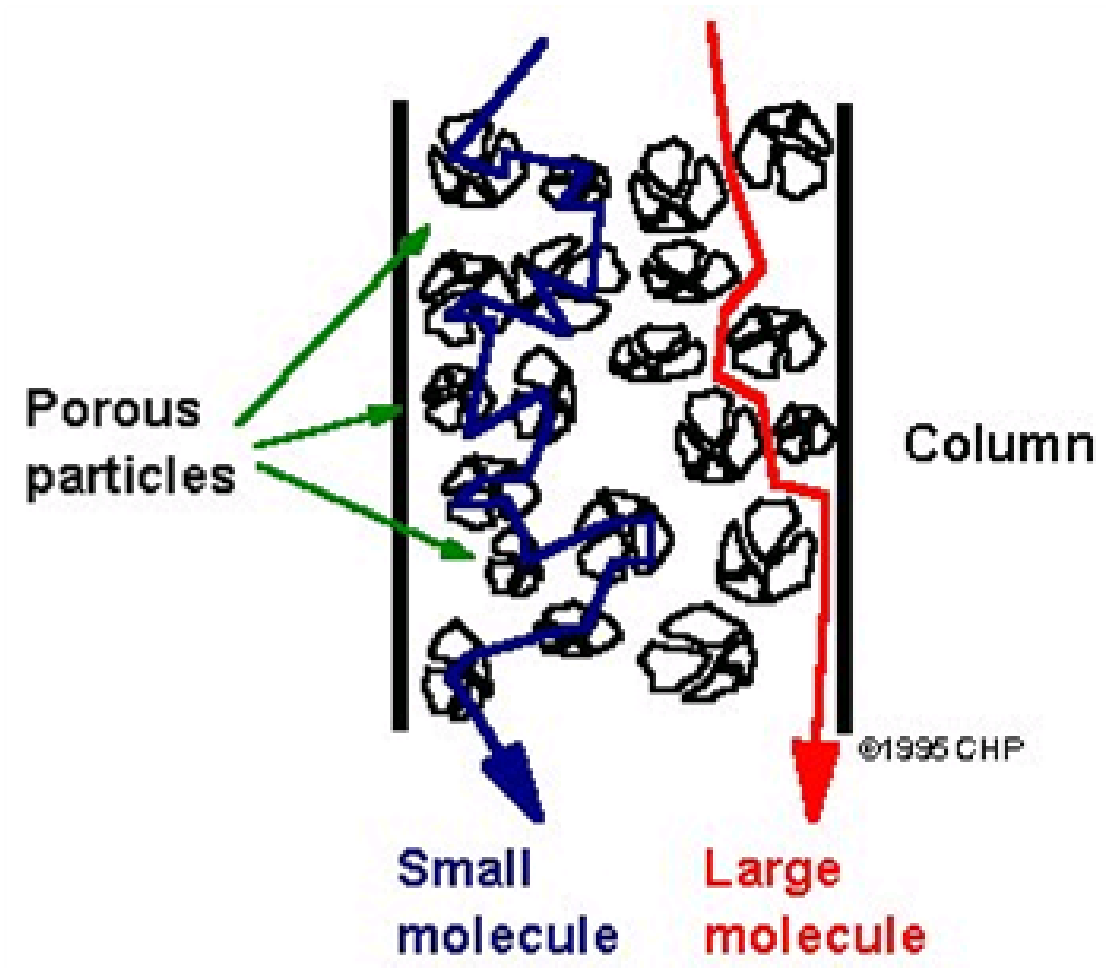

Figure 1-4: The mechanism of a size exclusion column chromatography.

\section{1-8. Dry eye}

\section{1-8-1. Mechanism of dry eye}

Dry eye is a condition where there is a problem with the production of tears. Tears are important as they form a layer on the very front of the eye. Called the tear film, this layer does a number of things. The tear film takes a part in the focusing of light into the eye however it mainly lubricates the eye, keeping it moist and smooth. The tear film is actually made up of three layers each with a different function. The layer closest to the eye is called the mucin layer. It coats the cornea which is the clear window at the front of the eye; it forms a foundation for the other layers of the tear film. The middle layer is called the aqueous layer. This is the watery layer that most people think of when they think of tears. This layer is produced by the lacrimal gland 
which is underneath the upper eyelid. This layer provides moisture and supplies oxygen and other important nutrients to the cornea. This layer is mainly water. The outer layer is called the lipid layer. This is an oily film which seals the tear film to the eye and helps to prevent any evaporation. The mucin and lipid layers are produced by small glands around the eye. Each time we blink the tears are spread onto the front of the eye. Excess tears are drained away by tiny drainage holes on the inside of the eyelids, these holes channel the excess tears into the nose. If you have dry eye usually eyes feel irritated, scratchy, dry and uncomfortable. Eyes may be red and there may be a burning sensation, or it may feel as if there is something in the eye like grit or an eyelash. Sometimes there may be periods of blurred vision.

\section{1-8-2. Causes of dry eye}

Dry eye has a number of causes. It happens mostly as a part of the natural ageing process but can also be caused by problems with blinking or problems with the glands which produce the tears. Some drugs can cause dry eye, like antihistamines and oral contraceptives. Contact lenses carry an increased risk if one have dry eye. Sometimes dry eye is also a symptom of other conditions affecting other parts of the body particularly arthritis or a condition called Sjogrens syndrome. Sjogrens syndrome is a condition where, as well as dry eyes, people can also have a dry mouth and a dry vagina. This condition can also involve a type of arthritis.

\section{1-8-3. Dry eye diagnosis}

Usually there are three main tests to work out how dry the eyes are. These tests can give the idea of treatment. The tests can check how many tears are produced and detect any areas on the front of the eye that doesn't have enough tears. One of the main tests is called tear film break-up time. This test can find out how long after blinking the eye starts to dry out. Usually eye-drops with a special dye are put in the eye and then after few times of blinking the eye must be kept open without blinking. Then a coloured light is used to see the dye and the period between the last blink and the formation of dry patches is measured.

Healthy eyes are continuously covered by fluid. A tear film designed to remain stable between blinks. A stable tear film prevents dry eye and allows your eyes to maintain 
clear, comfortable vision. In a stable tear film, the three layers, mucin layer, aqueous layer and lipid layer, remain stable and in good condition. Therefore the investigation of these three layers of the tear film is very important for dry eye diagnosis. A numbers of organic dyes such as fluorescein and rose Bengal have been used to mark the layers, however quantum dots have been introduced as new fluorophore because of their much better photo-stability. Since CdSe quantum dots have an issue with bio compatibility as described previously in other chapters and silicon quantum dots were not suitable for the tear film marker because of their limited emission peak shift, organic dye doped silica nanoparticles have become a potential candidate for this application. ${ }^{94-101}$

\section{1-9. Silica nanoparticles}

Silica nanoparticles can be synthesised by microemulsion synthesis by using surfactants such as triton-X 100 . Most of the chemicals are relatively easy to obtain with reasonable cost. Already a few studies have been carried out on dye doping methods. Mohr et. al. reported rhodamine doped silica nanoparticles using silylated sulforhodamine derivative ${ }^{102}$. They also modified the surface of silica nanoparticles with aminopropyltriethoxysilane (APTES) to form amine terminated particles. They further attached $\mathrm{pH}$ sensitive naphthalimide derivative on to the surface using the amine group. Tris (2,2'-bipyridine) dichlororuthenium (II) hexahydrate (Rubpy) doped silica nanoparticles were reported by Tan et. $\mathrm{al}^{103}$. Since Rubpy can react with tetraethyl orthosilicate (TEOS) directly the method of this synthesis was quite straightforward. Simard et. al. reported rhodamine doped silica particles by using tetramethylrhodamine-5-isothiocyanate (TRITC) and APTES ${ }^{104}$. Optical properties and photo-stability were reported by Yang et al. and they claim that encapsulating the organic dye with silica particles overcomes the photo-bleaching problem of organic dyes and shows better photo-stability ${ }^{105}$. 


\section{1-10. References}

1. A. Alivisatos; J. Phys. Chem., 1996, 100, 13226-13239, "Perspectives on the Physical Chemistry of Semiconductor Nanocrystals”

2. A. Alivisatos; Science, 1996, 271, 933-937, "Semiconductor clusters, nanocrystals, and quantum dots”

3. M. Bruchez Jr., M. Moronne, P. Gin, S. Weiss, A. Alivisatos; Science, 1998, 281, 2013-2016, “Semiconductor Nanocrystals as Fluorescent Biological Labels”

4. W. C. W. Chan and S. Nie; Science, 1998, 281, 2016-2018, "Quantum Dots Biocondugates for Ultrasensitive Nonisotopic Detection”

5. H. Mattousi, J. M. Mauro, E. R. Goldman, G. P. Anderson, V. C. Sundar, F. V. Mikulec, and M. G. Bawendi; J. Am. Chem. Soc., 2000, 122, 12142-12150, "Self-Assembly of CdSe-ZnS Quantum Dots Biocondugates Using an Engineered Recombinant Protein”

6. B. Dubertret, P. Skourides, D. J. Norris, V. Noireaux, A. H. Brivanlou, and A. Libchaber; Science, 2002, 298, 1759-1762, "In Vivo imaging of Quantum Dots Encapsulated in Phospholipid Micelles”

7. A. Cazet, L. Guyon, P. Delannoy, F. Vinet, D. Bonnaffe, and I. Texier; Bioconjugate Chem. 2010, 21, 583-588, “Copper-Free Click Chemistry for Highly Luminescent Quantum Dot Conjugates: Application to in Vivo Metabolic Imaging”

8. E. Muro, T. Pons, N. Lequeux, A. Fragola, N. Sanson, Z. Lenkei, and B. Dubertre; J. Am. Chem. Soc. 2010, 132, 4556-4557, "Small and Stable Sulfobetaine Zwitterionic Quantum Dots for FunctionalLive-Cell Imaging” 
9. W. Zhou, D. T. Schwartz, and F. Baneyx; J. Am. Chem. Soc. 2010, 132, 47314738, "Single-Pot Biofabrication of Zinc Sulfide Immuno-Quantum Dots"

10. D. Thakur1, S. Deng, T. Baldet, and J. O. Winter; Nanotechnol., 2009, 20, 485601, "pH sensitive CdS-iron oxide fluorescent-magnetic nanocomposites"

11. R. Kas, E. Sevinc, U. Topal, and H. Y. Acar; J. Phys. Chem. C, 2010, 114, 7758-7766, “A Universal Method for the Preparation of Magnetic and Luminescent Hybrid Nanoparticles”

12. A. Alivisatos; Nat. Biotechnol., 2004, 22, 47-52, "The use of nanocrystals in biological detection”

13. K. Cheng, S. Peng, C. Xu, and S. Sun; J. Am. Chem. Soc., 2009, 131, 1063710644, "Porous Hollow Fe3O4 Nanoparticles for Targeted Delivery and Controlled Release of Cisplatin”

14. B. Y. S. Kim, W. Jiang, J. Oreopoulos, C. M. Yip, J. T. Rutka, and W. C. W. Chan; Nano Lett., 2008, 8, 3887-3892, "Biodegradable Quantum Dot Nanocomposites Enable Live Cell Labeling and Imaging of Cytoplasmic Targets”

15. Y. He, H. T. Lu, L. M. Sai, Y. Y. Su, M. Hu, C. H. Fan, W. Huang, and L. H. Wang; Adv. Mater., 2008, 20, 3416-3421, "Microwave synthesis of waterdispersed $\mathrm{CdTe} / \mathrm{CdS} / \mathrm{ZnS}$ core-shell-shell quantum dots with excellent photostability and biocompatibility”

16. A. Hoshino, K. Hanaki, K. Suzuki, K. Yamamoto; Biochem. Biophys. Res. Commun., 2004, 314, 46-53, “Applications of T-lymphoma labelled with fluorescent quantum dots to cell tracing markers in mouse body”

17. K. Hanaki, A. Momo, T. Oku, A. Komoto, S. Maenosono, Y. Yamaguchi, and K. Yamamoto; Biochem. Biophys. Res. Commun., 2003, 302, 496-501, 
"Semiconductor quantum dots/albumin complex is a long-life and highly photostable endosome marker”

18. D. Gerion, F. Pinaud, S. C. Williams, W. J. Parak, D. Zanchet, S. Weiss, and A. Alivisatos; J. Phys. Chem. B., 2001, 105, 8861-8871, "Synthesis and Properties of biocompatible Water-Soluble Silica-coated CdSe/ZnS Semiconductor Quantum Dots”

19. A. Shiohara, N. Manabe, K. Omata, and K. Yamamoto; J. Chem. Eng. Jpn., 2006, 39, 52-56, "Novel Surface Processing with Sufonic Acid for Quantum Dot and Its characteristics"

20. S. Coe, W. K. Woo, M. Bawendi, and V. Bulovic; Nature, 2002, 420, 800-803, "Electroluminescence from single monolayers of nanocrystals in molecular organic devices”

21. C. Santori, D. Fattal, J. Vuckovic, G. S. Solomon, and Y. Yamamoto; Nature, 2002, 419, 594-597, "Indistinguishable photons from a single-photon device"

22. T. C. Harman, P. J. Taylor, M. P. Walsh, B.E. LaForge; Sicence, 2002, 297, 2229-2232, “Quantum Dot Superlattice Thermoelectric Materials and Devices”

23. X. Li, Y. Wu, D. Steel, D. Gammon, T. H. Stievater, D. S. Katzer, D. Park, C. Piermarocchi, and L. J. Sham; Sicence, 2003, 301, 809-811, “An All-Optical Quantum Gate in a Semiconductor Quantum Dot”

24. E. Beham, S. Stufler, F. Findeis, M. Bichler, G. Abstreiter, A. Zrenner; Nature, 2002, 418, 612-614, "Coherent properties of a two-level system based on a quantum-dot photodiode”

25. X. Wu, H. Liu, J. Liu, K. N. Haley, J. A. Treadway, J. P. Larson, N. Ge, F. Peale, and M. P. Bruchez; Nature Biol., 2003, 21, 41-46, “Immunofluorescent 
labeling of cancer marker Her2 and other cellular targets with semiconductor quantum dots”

26. M. Nirmal, B. O. Dabbousi, M. G. Bawendi, J. J. Macklin, J. K. Trautman, T. D. Harris, L. E. BrusJournal; Nanotechnol, 1996, 383, 802-804, "Fluorenscence Intermittency in single cadmium selenide nanocrystals"

27. D. Neiner, H. W. Chiu, S. M. Kauzlarich; J. Am. Chem. Soc., 2006, 128, 11016-11017, “Low-Temperature Solution Route to Macroscopic Amounts of Hydrogen Terminated Silicon Nanoparticles”

28. R. K. Baldwin, J. Zou, K. A. Pettigrew, G. J. Yeagle, R. D. Britt, S. M. Kauzlarich; Chem. Comm., 2006, 6, 658-660, "The preparation of a phosphorus doped silicon film from phosphorus containing silicon nanoparticles”

29. J. Zou, R. K. Baldwin, K. A. Pettigrew, S. M. Kauzlarich; Nano Lett., 2004, 4, 1181-1186, "Solution Synthesis of Ultrastable Luminescent Siloxane-Coated Silicon Nanoparticles”

30. Q. Liu, S. M. Kauzlarich; Mater. Sci. Eng. B, 2002, 96, 72-75, “A new synthetic route for the synthesis of hydrogen terminated silicon nanoparticles”

31. D. Neiner, and S. M. Kauzlarich; Chem. Mater., 2010, 22, 487-493, "Hydrogen-Capped Silicon Nanoparticles as a Potential Hydrogen Storage Material; Synthesis, Characterization, and Hydrogen Release”

32. R. K. Baldwin, K. A. Pettigrew, E. Ratai, M. P. Augustine, S. M. Kauzlarich; Chem. Comm., 2002, 17, 1822-1823, "Solution reduction synthesis of surface stabilized silicon nanoparticles”

33. R. K. Baldwin, K. A. Pettigrew, J. C. Garno, P. P. Power, G. Y. Liu, S. M. Kauzlarich; J. Am. Chem. Soc., 2002, 124, 1150-1151, "Room Temperature Solution Synthesis of Alkyl-Capped Tetrahedral Shaped Silicon Nanocrystals” 
34. D. Mayeri, B. L. Phillips, M. P. Augustine, S. M. Kauzlarich; Chem. Mater., 2001, 13, 765-770, "NMR Study of the Synthesis of Alkyl-Terminated Silicon Nanoparticles from the Reaction of $\mathrm{SiCl}_{4}$ with the Zintl Salt, $\mathrm{NaSi}$ ”

35. C. S. Yang, R. A. Bley, S. M. Kauzlarich, H. W. H. Lee, G. R. Delgado; J. Am. Chem. Soc., 1999, 121, 5191-5195, "Synthesis of Alkyl-Terminated Silicon Nanoclusters by a Solution Route”

36. R. A. Bley, S. M. Kauzlarich; J. Am. Chem. Soc., 1996, 118, 12461-12462, “A Low-Temperature Solution Phase Route for the Synthesis of Silicon Nanoclusters”

37. D. C. Lee, T. Hanrath, B. A. Korgel; Angew. Chem Int Ed., 2005, 44, 35733577, "The Role of Precursor-Decomposition Kinetics in Silicon-Nanowire Synthesis in Organic Solvents”

38. L. E. Pell, A. D. Schricker, F. V. Mikulec, B. A. Korgel; Langmuir, 2004, 20, 6546-6548, "Synthesis of Amorphous Silicon Colloids by Trisilane Thermolysis in High Temperature Supercritical Solvents"

39. D. S. English, L. E. Pell, Z. H. Yu, P. F. Barbara, B. A. Korgel; Nano Lett., 2002, 2, 681-685, "Size Tunable Visible Luminescence from Individual Organic Monolayer Stabilized Silicon Nanocrystal Quantum Dots”

40. J. D. Holmes, K. J. Ziegler, R. C. Doty, L. E. Pell, K. P. Johnston, B. A. Korgel; J. Am. Chem. Soc., 2001, 123, 3743-3748, "Highly Luminescent Silicon Nanocrystals with Discrete Optical Transitions”

41. D. Zhifeng, M. Q. Bernadette, K. H. Santosh, L. E. Pell., B. A. Korgel., and J. B. Allen; Science, 2002, 296, 1293-1297, "Electrochemistry and Electrogenerated Chemiluminescence from Silicon Nanocrystal Quantum Dots” 
42. J. P. Wilcoxon, G. A. Samara, P. N. Provencio; Phys. Rev. B, 1999, 60, 27042714, "Optical and electronic properties of Si nanoclusters synthesized in inverse micelles”

43. J. P. Wilcoxon, G. A. Samara; Appl. Phys. Lett., 1999, 74, 3164-3166, “Tailorable, visible light emission from silicon nanocrystals”

44. A. M. Derfus, W. C. W. Chan, S. N. Bhatia; Nano Lett., 2004, 4, 11-18, "Probing the Cytotoxicity of Semiconductor Quantum Dots"

45. P. Wright; Churchill College Cambridge, 20 September, 2000 "Quantum confinement effects in semiconductor clusters"

46. H. S. Mavi, A. K. Shukla, R. Kumar, S. Rath, B. Joshi, and S. S. Islam; Semicond. Sci. Technol., 2006, 21, 1627-1632, “Quantum confinement effects in silicon nanocrystals produced by laser-induced etching and cw laser annealing”

47. T. Takagahara, K. Takeda; Phys. Rev. B., 1992, 46, 15578-15581, “Theory of the quantum confinement effect on excitons in quantum dots of indirect-gap materials”

48. Research Centre for Quantum Effect Electronics (Tokyo Institute of Technology); http://www.electrochem.org/dl/ma/201/pdfs/1207.pdf

49. M. Sitti, H. Hashimoto; IEEE/ASME Trans. Mechatron., 2003, 8, 287-298, “Teleoperated Touch Feedback From the Surfaces at the Nanoscale: Modeling and Experiments”

50. Y. M. Wan, H. T. Lin, C. L. Sung, S. F. Hu; Appl. Phys. Lett., 2005, 87, 123506, "Nanopillar transistors exhibiting single-electron quantum effects at room temperature” 
51. J. H. Warner, R. D. Tilley; Adv. Mater, 2005, 17, 2997-3001, "Synthesis and Self-Assembly of Triangular and Hexagonal CdS Nanocrystals”

52. http://ece.colorado.edu/ bart/book/book/chapter2/ch2 3.htm\#fig2 33

53. H. W. Chiu, S. M. Kauzlarich; Chem. Mater., 2006, 18, 1023-1028, "Investigation of Reaction Conditions for Optimal Germanium Nanoparticle Production by a Simple Reduction Route”

54. R. S. Tanke, S. M. Kauzlarich, T. E. Patten, K. A. Pettigrew, D. L. Murphy, M. E. Thompson, H. W. H. Lee; Chem. Mater., 2003, 15, 1682-1689, "Synthesis of Germanium Nanoclusters with Irreversibly Attached Functional Groups: Acetals, Alcohols, Esters, and Polymers”

55. X. M. Lu, B. A. Korgel, K. P. Johnston; Nanotechnology, 2005, 16, S389S394, "Synthesis of germanium nanocrystals in high temperature supercritical $\mathrm{CO}_{2} ”$

56. T. Hanrath, B. A. Korgel; Adv. Mater., 2003, 15, 437-440, "Supercritical Fluid-Liquid-Solid (SFLS) Synthesis of Si and Ge Nanowires Seeded by Colloidal Metal Nanocrystals”

57. J. P. Wilcoxon, P. P. Provencio, G. A. Samara; Phys. Rev. B, 2001, 64, Art. No. 035417, "Synthesis and optical properties of colloidal germanium nanocrystals”

58. M. Mitsubaga, T. Takagahara, R. Yano, N. Uesugi; Phys. Rev., Lett., 1992, 68, 3216-3219, "Excitation-induced frequency shift probed by stimulated photon echoes"

59. D. Zhifeng, M. Q. Bernadette, K. H. Santosh, L. E. Pell., B. A. Korgel., and J. B. Allen; Science, 2002, 296, 1293-1297, "Electrochemistry and Electrogenerated Chemiluminescence from Silicon Nanocrystal Quantum Dots” 
60. R. D. Tilley, J. H. Warner, K. Yamamoto, I. Matsui, and H. Fujimori; Chem. Commun., 2005, 14, 1833-1835, "Micro-emulsion synthesis of monodisperse surface stabilized silicon nanocrystals”

61. J. H. Warner, A. Hoshino, K. Yamamoto, R. D. Tilley; Angew, 2005, 44, 4550-4554, "Water-Soluble Photoluminescent Silicon Quantum Dots”

62. A. Shiohara, S. Hanada, S. Prabakar, K. Fujioka, T. H. Lim, K. Yamamoto, P. T. Northcote, and R. D. Tilley; J. Am. Chem. Soc., 2010, 132, 248-253, "Chemical Reactions on Surface Molecules Attached to Silicon Quantum Dots”

63. R. H. James; Science, 1992, 258, 1131-1133, “A Liquid-Solution-Phase Synthesis of Crystalline Silicon”

64. B. R. Taylor, S. M. Kauzlarich, G. R. Delgado, H. W. H. Lee; Chem. Mater., 1999, 11, 2493-2500, "Solution Synthesis and Characterization of Quantum Confined Ge Nanoparticles”

65. R. H. James; J. Chem. Phys., 1994, 101, 1607-1615, “Germanium quantum dots: Optical properties and synthesis”

66. R. H. James, and K. L. Francoise; Chem. Phys. Lett., 1993, 208, 263-268, “A liquid solution synthesis of single crystal germanium quantum wires”

67. H. Miguez, and V. Fornés; Appl. phys. Lett., 1996, 69, 2347-2349, "Low - temperature synthesis of Ge nanocrystals in zeolite Y"

68. B. R. Taylor, S. M. Kauzlarich, H. W. H. Lee, G. R. Delgado; Chem. Mater., 1998, 10, 22-24, "Solution Synthesis of Germanium Nanocrystals Demonstrating Quantum Confinement” 
69. B. R. Taylor, S. M. Kauzlarich, H. W. H. Lee, G. R. Delgado; Chem. Mater., 1999, 11, 2493-2500, "Solution Synthesis and Characterization of Quantum Confined Ge Nanoparticles”

70. X. Ma, F. Wu, and S. M. Kauzlarich; J. Solid State Chem., 2008, 181, 16281633, “Alkyl-terminated crystalline Ge nanoparticles prepared from NaGe; Synthesis, functionalization and optical properties”

71. J. H. Warner, R. D. Tilley; Nanotechnol., 2006, 17, 3745-3749, "Synthesis of water-soluble photoluminescent germanium nanocrystals”

72. S. Prabakar, A. Shiohara, S. Hanada, K. Fujioka, K. Yamamoto, R. D. Tilley; Chem. Mater., 2010, 22, 482-486, "Size Controlled Synthesis of Germanium Nanocrystals by Hydride Reducing Agents and Their Biological Applications”

73. B. Ludolph, and M. A. Malik; Chem Commun., 1998, 17, 1849-1850 "Novel single molecule precursor routes for the direct synthesis of highly monodispersed quantum dots of cadmium or zinc sulfide or selenide”

74. N. Revaprasadu, M. A. Malik, P. O'Brien, M. M. Zulu, and G. Wakefield; J. Am. Chem. Soc., 1998, 8, 1885-1888, "Single-source molecular precursors for the deposition of zinc selenide quantum dots”

75. W. C. W. Chan, and S. Nie.; Science, 1998, 281, 2016-2018, “Quantum Dot Bioconjugates for Ultrasensitive Nonisotopic Detection”

76. D. Gerion, F. Pinaud, S. C. Williams, W. J. Parak, D. Zanchet, S. Weiss, A. P. Alivisatos; J. Phys. Chem. B., 2001, 105(37), 8861-8871, "Synthesis and Properties of Biocompatible Water-Soluble Silica-Coated CdSe/ZnS Semiconductor Quantum Dots”

77. E. R. Goldman, E. D. Balighian, H. Mattoussi, M. K. Kuno, J. M. Mauro, P. T. Tran, G. P. Anderson; J. Am. Chem. Soc., 2002, 124, 6378-6382, “Avidin: A Natural Bridge for Quantum Dot-Antibody Conjugates” 
78. E. R. Goldman, G. P. Anderson, P. T. Tran, H. Mattoussi, P. T. Charles, J. M. Mauro; Anal. Chem., 2002, 74, 841-847, “Conjugation of Luminescent Quantum Dots with Antibodies Using an Engineered Adaptor Protein To Provide New Reagents for Fluoroimmunoassays”

79. S. H. Hwang, C. N. Moorefield, P. Wang, K. U. Jeong, S. D. Z. Cheng, K. K. Kotta, and G. R. Newkome; Chem. Commun., 2006, 33, 3495-3497, "Construction of CdS quantum dots via a regioselective dendritic functionalized cellulose template”

80. K. Hanaki, A. Momo, T. Oku, A. Komoto, S. Maenosono, Y. Yamaguchi, and K. Yamamoto; Biochem. Biophys. Res Commun, 2003, 302, 496-501, "Semiconductor quantum dot/albumin complex is a long-life and highly photostable endosome marker”

81. A. M. Derfus, W. C. W. Chan, S. N. Bhatia; Nano Lett., 2004, 4(1), 11-18, "Probing the Cytotoxicity of Semiconductor Quantum Dots"

82. Z. F. Li, E. Ruckenstein; Nano Lett., 2004, 4, 1463-1467, "Water-Soluble Poly(acrylic acid) Grafted Luminescent Silicon Nanoparticles and Their Use as Fluorescent Biological Staining Labels”

83. S. Sato, M. T. Swihart; Chem. Mater., 2006, 18, 4083-4088, "Propionic-AcidTerminated Silicon Nanoparticles: Synthesis and Optical Characterization”

84. F. Erogbogbo, K. T. Yong, I. Roy, G. Xu, P. N. Prasad, and M. T. Swihart; ACS Nano, 2008, 2, 873-878, “Bioconpatible Luminescent Silicon Quantum Dots for Imaging of Cancer Cells”

85. A. Gupta, M. T. Swihart, and H. Wiggers; Adv. Funct. Mater., 2009, 19, 696703, “Luminescent Colloidal Dispersion of Silicon Quantum Dots from Microwave Plasma Synthesis: Exploring the Photoluminescence Behavior Across the Visible Spectrum” 
86. M. P. Stewart, and J. M. Buriak; J. Am. Chem. Soc., 2001, 123, 7821-7830, "Exciton-Mediated Hydrosilylation on Photoluminescent Nanocrystalline Silicon”

87. K. M. Krueger, A. M. Al-Somali, J. C. Falkner, and V. L. Colvin; Anal. Chem., 2005, 77, 3511-3515, "Characterization of Nanocrystalline CdSe by Size Exclusion Chromatography”

88. W. W. Yu, E. Chang, J. C. Falkner, J. Zhang, A. M. Al-Somali, C. M. Sayes, J. Johns, R. Drezek, and V. L. Colvin; J. Am. Chem. Soc., 2007, 129, 2871-2879, "Forming Biocompatible and Nonaggregated Nanocrystals inWater Using Amphiphilic Polymers”

89. L. Wenguang, Q.X. Jia, and W. Hsing-Lin; Polymer, 2006, 47(1), 23-26, "Facile synthesis of metal nanoparticles using conducting polymer colloids"

90. D. Gerion, N. Zaitseva, C. Saw, M. F. Casula, S. Fakra, T. van Buuren, G. Galli; Nano Lett., 2004, 4, 597-602, "Solution Synthesis of Germanium Nanocrystals: Success and Open Challenges”

91. X. Lu, B. A. Korgel, K. P. Johnston; Chem. Mater., 2005, 17, 6479-6485, "High Yield of Germanium Nanocrystals Synthesized from Germanium Diiodide in Solution”

92. http://en.wikipedia.org/wiki/Chromatography\#Sizeexclusion_chromatography

93. http://www.chemistry.adelaide.edu.au/external/soc-rel/content/size-exc.htm

94. http://www.allaboutvision.com/conditions/dryeye.htm

95. http://arthritis.about.com/od/sjogrens/a/guidetodryeyes_2.htm 
96. http://www.docshop.com/education/vision/eye-disorders/dry-eye/diagnosis/

97. http://en.wikipedia.org/wiki/Fluorescein

98. A. S. Kwan, C. Barry, I. L. McAllister, I. Constable; Clin. Experiment. Ophthalmol., 2006, 34, 33-8, "Fluorescein angiography and adverse drug reactions revisited: the Lions Eye experience”

99. http://www.rnib.org.uk/eyehealth/eyeconditions/eyeconditionsdn/Pages/dry_e ye.aspx

100. A. J. Bron BSc, FRCOphth; Survey of ophthalmology, 2001, 45, S221S226, "Diagnosis of Dry Eye”

101. S. Khanal, A. Tomlinson, A. McFadyen, C. Diaper, and K. Ramaesh; IOVS, 2008, 49, 1407-1414, "Dry Eye Diagnosis"

102. T. Doussineau, S. Trupp, G. J. Mohr; J. Colloid Interface Sci., 2009, 339, 266-270, "Ratiometric pH-nanosensors based on rhodamine-doped silica nanoparticles functionalized with a naphthalimide derivative”

103. R. P. Bagwe, C. Yang, L. R. Hilliard, and W. Tan; Langmuir, 2004, 20, 8336-8342, “Optimization of Dye-Doped Silica Nanoparticles Prepared Using a Reverse Microemulsion Method”

104. D. Ma, A. J. Kell, S. Tan, Z. J. Jakubek, and B. Simard; J. Phys. Chem. C, 2009, 113, 15974-15981, "Photophysical Properties of Dye-Doped Silica Nanoparticles Bearing Different Types of Dye-Silica Interactions”

105. W. Yang, C. G. Zhang, H. Y. Qu, H. H. Yang, and J. G. Xu; Anal. Chim. Acta, 2004, 203, 163-169, "Novel fluorescent silica nanoparticle probe for ultrasensitive immunoassays” 


\section{Chapter 2 Characterisation techniques}

In this chapter characterisation techniques are described which were applied to the samples in this research. TEM/HRTEM (Transmission Electron Microscopy/HighResolution Transmission Electron Microscopy), EDS (Energy Dispersive X-ray Spectroscopy) and SAED (Selected Area Electron Diffraction) were employed to characterise size, shape and the crystallinity of the samples, respectively. FTIR (Fourier Transform Infrared Spectroscopy) and NMR (Nuclear Magnetic Resonance Spectroscopy) were applied to characterise the surface molecules of the quantum dots. UV-Vis (Ultraviolet-visible Spectroscopy) and PL (Photoluminescence Spectroscopy) were used to measure optical properties of the samples. For biological applications, a cell viability assay was applied to evaluate cyto-toxicity of the quantum dots.

\section{2-1. High Resolution Transmission Electron Microscopy (HRTEM)}

The TEM sample was prepared on carbon coated 200 mesh copper grid purchased from ProScitech. A droplet of diluted solution of the sample was placed onto the grid surface and solvent was dried out under lamp for 20 minutes.

\section{2-1-1. Basic theory of TEM (Transmission Electron Microscopy)}

TEM is an electron microscopy technique whereby an electron beam is transmitted through an ultra thin specimen. When the electrons pass through the specimen they are scattered by atoms in the sample. Those scattered electrons convey information on the internal structure of samples, which can be observed as electron diffraction patterns or transmission electron microscope images.

An important account of the technique can be found in the reference by D. Williams and C. Barry Carter ${ }^{1,2}$. However, the current description of TEM is based on the doctoral thesis of R.D. Tilley ${ }^{3}$. The main components of an electron microscope are 
illustrated in Figure 2-1. The TEM used in this research was a JEOL 2010 with a standard self-biasing thermionic $\mathrm{LaB}_{6}$ electron source. In standard operation, the electrons were accelerated by an applied potential difference of $200 \mathrm{kV}$. These fast moving electrons have wavelengths ( $\lambda=0.0251 \AA$ ) much smaller than inter-atomic distances in crystal lattices. Therefore when the electron beam interacts with a crystal, the electrons are scattered by the atoms and give diffraction patterns containing information on crystal structure of material.

Due to the relativistic effects on the electron caused by the acceleration by the large potential difference, the precise wavelength of the electrons is given by equation 2-1

$$
\lambda=\frac{h\left(1-\beta^{2}\right)^{1 / 4}}{\left(2 m_{e} e_{0} E\right)^{1 / 2}} \quad \text { (eq. 2-1) }
$$

Where $h$ is Plank's constant, $m_{e}$ is the rest mass of the electron, $e_{0}$ is the electronic charge, $E$ is the accelerating voltage and $\beta=v / c$, where $v$ is the velocity of the electron and $c$ is the velocity of light in a vacuum. 


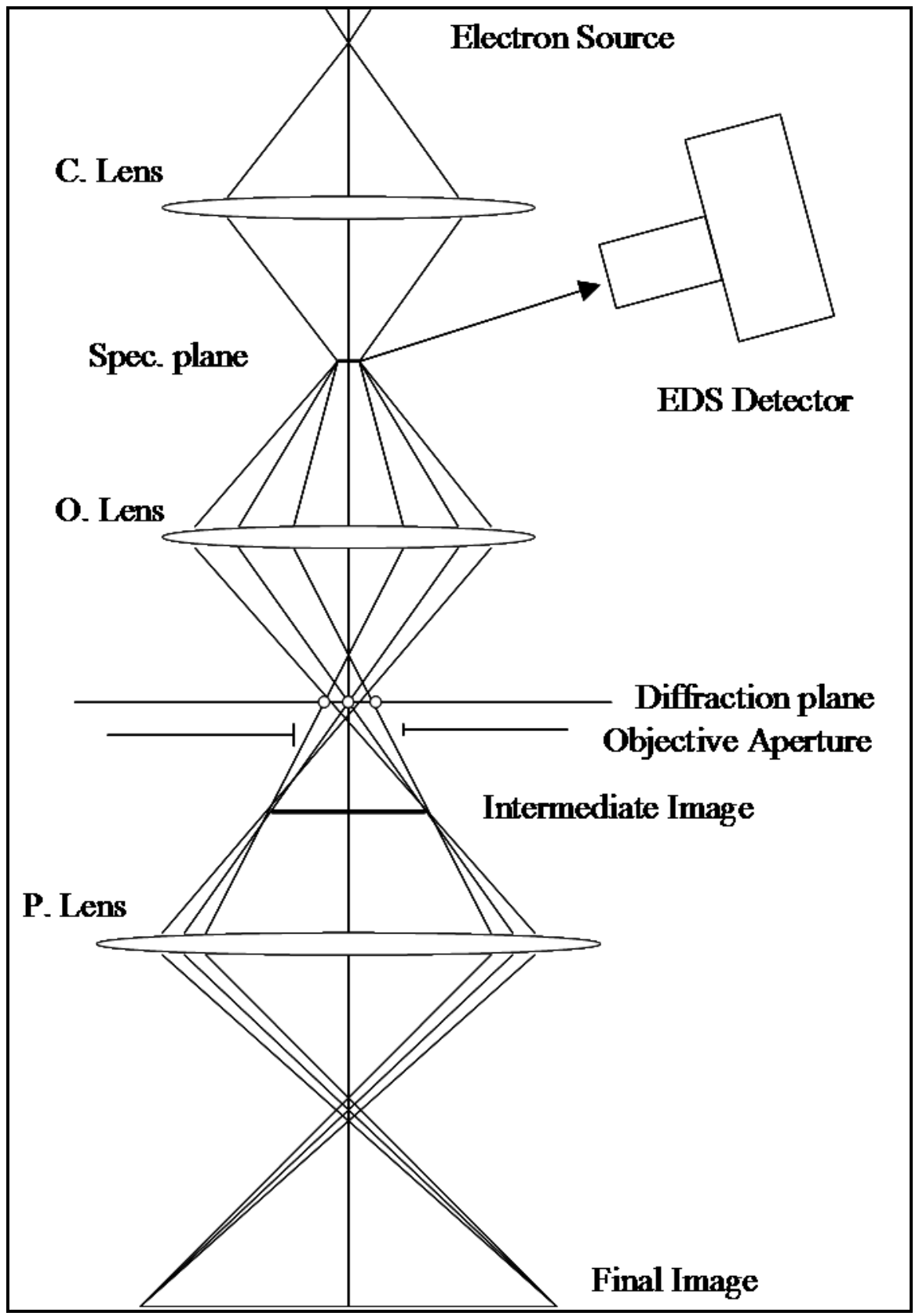

Figure 2-1: A schematic diagram of a transmission electron microscope

In the TEM the electrons accelerated by the potential difference are first focused by the condenser lenses to form an approximately parallel beam. This beam then strikes the sample. Some of the electrons will be diffracted by sample, imparting structural information to the beam. The diffracted beam and the undiffracted, or primary beam, come out through the sample and are recombined by the objective lens. The electron beam wavefronts are focused to a point which lies on a plane, normal to the beam (perpendicular to the beam), called the back focal plane of the objective lens. This 
gives an (electron) diffraction pattern, which is in effect a Fourier transform of the electron density in the crystal. The other lenses complete an inverse Fourier transform to give an "image" of the sample. By alternation of the strength of the projector lenses, either the image or the electron diffraction pattern can be transferred down the microscope and be recorded on a fluorescent screen, films or captured by a digital camera. By changing objective aperture differing image contrast can be obtained.

There are some important advantages of electron microscope over other techniques. Since the strength of interaction of electrons with atoms are much greater compared to X-rays, it makes possible to obtain diffraction patterns from higher Laue zones.

However, there are some limitations on the structural resolution that can be obtained because of imperfections of the limiting factors. These limiting factors are mainly due to objective lens. The lens aberrations of the objective lens are so great that it is necessary to work with very small objective apertures to achieve resolution of the order of $0.2-0.5 \mathrm{~nm}$.

\section{2-1-2. Scattering and Diffraction}

As it is described above when electrons travel through the specimen, they are either transmitted unscattered or they can be scattered elastically (no energy transferred during scattering) or inelastically (energy transferred between electron and sample during scattering). The end result is that a nonuniform distribution of electrons emerges from the both upper and lower surface of the specimen, as shown in Figure 2-2. This nonuniform distribution contains all the structural and chemical information about the specimen imparted to the electron beam. The electron microscope is constructed to display this nonuniform distribution of electrons in two different ways. First, the angular distribution of scattering can be viewed in the form of scattering patterns, usually known as diffraction patterns. The second one is the spatial distribution of scattering can be observed as contrast in images of the specimen. A simple operation step in TEM is to use a restricting aperture, or an electron detector, of a size such that only electrons that have suffered more or less than a certain angular deviation are selected. Thus an operator can choose which 
electrons are used and control what information will be displayed in the image or diffraction pattern.

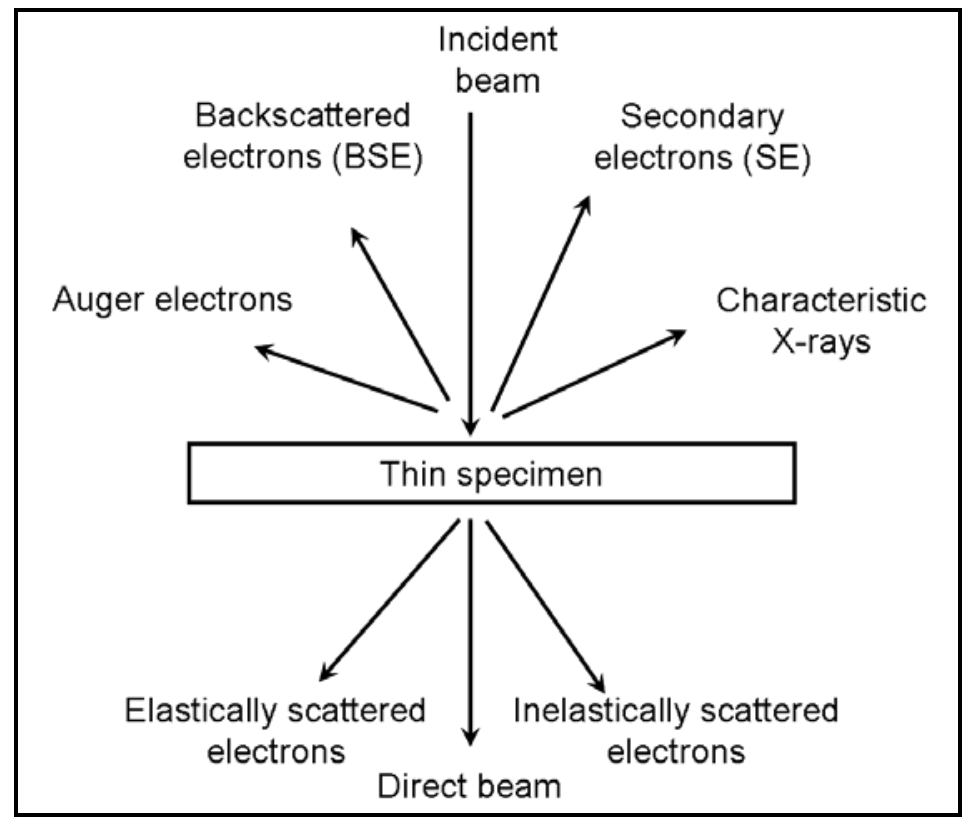

Figure 2-2: Different kinds of electron scattering from a thin specimen

\section{Elastic scattering}

\section{A. Mechanisms of elastic scattering}

It is convenient to divide elastic scattering mechanisms into two principal forms. One is electron scattering from isolated single atoms and the other one is collective scattering from many atoms together within the specimen. Both ways involve Coulomb forces. As shown in Figure 2-3, the electron may interact with the electron cloud, resulting in a small angular deviation. Alternatively, if an electron penetrates the electron cloud and approaches the nucleus, it will be strongly attracted and may be strongly scattered through a larger angle.

The second principal form of elastic scattering occurs when the electron wave interacts with the specimen as a whole. To understand this principle it is important to consider the electron beam as a wave. Each atom in the specimen acts as a source of “secondary” spherical wavelets. These wavelets reinforce one another in certain angular directions and cancel in others. Thus the low angle elastic scattering distribution is modified by the crystal structure of the specimen and intense diffracted beams emerge at certain specific angles. 


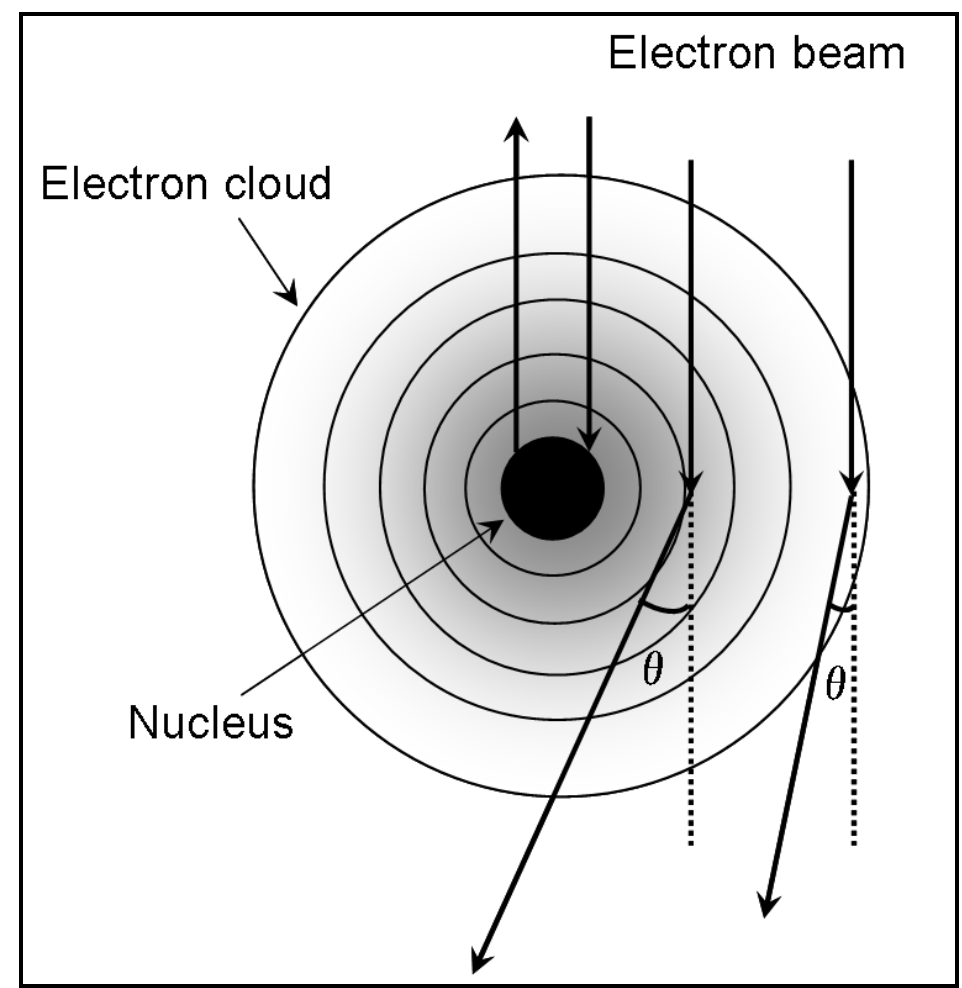

Figure 2-3: Mechanisms by which a high-energy electron is scattered by an isolated atom

As it is shown in Figure 2-3, when an electron passes close to an atomic nucleus the direction travelled by the electron changes through an angle $\theta$. Elastic electronelectron interactions usually result in a relatively low scattering angle, while electronnucleus interactions cause higher-angle scattering. If only an electron scattering from an isolated atom is considered, the electron-electron and electron-nucleus scattering cross sections which are the probabilities that an electron will be scattered by another electron or a nucleus respectively can be easily expressed by two very simple equations. (eq. 2-2)

$$
\begin{aligned}
& \sigma_{\text {electron }}=\pi r_{e}^{2}=\pi\left(\frac{e}{V \theta}\right)^{2} \\
& \sigma_{\text {nucleus }}=\pi r_{n}^{2}=\pi\left(\frac{Z e}{V \theta}\right)^{2}
\end{aligned}
$$

Where $\sigma$ is the scattering cross section, $e$ is the charge of an electron, $\mathrm{Z}$ is the mass number of the nucleus. In electron-nucleus scattering, the atomic number $\mathrm{Z}$ of the atom controls the elastic interaction with the nucleus, but the electron-electron 
scattering is more function of the incident beam energy $(V)$. The strong $\mathrm{Z}$ effect of electron-nucleus scattering becomes important to enhance scattering in low-Z materials to get better TEM contrast.

\section{B. The atomic scattering factor}

The most familiar aspect of the wave approach of electrons to a cross section is the concept of the atomic scattering factor $f(\theta)$. The atomic scattering factor is related to the differential elastic scattering cross section. $\Omega$ is a total solid angle of scattering. (eq. 2-3)

$$
|f(\theta)|^{2}=\frac{d \sigma(\theta)}{d \Omega}
$$

The scattering factor approach is complementary to the Rutherford differential cross section, because it is most useful for describing the low-angle elastic scattering where the Rutherford model is inappropriate. Usually, the atomic scattering factor is defined in the following manner. (eq. 2-4)

$$
f(\theta)=\frac{\left(1+\frac{E}{E_{0}}\right)}{8 \pi^{2} a_{0}}\left(\frac{\lambda}{\sin \frac{\theta}{2}}\right)^{2}(Z-f x)
$$

The wavelength is $\lambda$ and $f x$ is the scattering factor for x-rays which is well known. $E$ and $E_{0}$ are the accelerating boltage and the energy of electrons respectively. $a_{0}$ is the Bohr radius of the scattering atom. As may be seen, $f(\theta)$ depends on $\lambda, \theta$ and $\mathrm{Z}$. It decreases as $\theta$ increases and also as $\lambda$ decreases. It increases with $\mathrm{Z}$ at any value of $\theta$.

\section{2-1-3. TEM image of crystalline nanoparticles}

Due to the factors mentioned in the section above, contrast in TEM images can originate due to spatial variation in the Z-number within a sample (i.e. variation in composition). Areas with higher average Z-number tend to scatter electrons more 
strongly, and therefore appear darker than lower Z-number areas in bright-field images ( $\mathrm{Z}$ contrast). This effect is also proportional to the thickness of the specimen, with thicker areas scattering more intensely and appearing darker (thickness contrast).

TEM can provide the information of the sample as an image. In the TEM image, size, size distribution of the products, and shape can be observed. At high magnification of the crystalline sample, the lattice fringes of the material can also be observed which gives information on the atomic packing of the nanocrystal. The TEM image of silicon quantum dots at low and high magnification is shown in Figure 2-4.

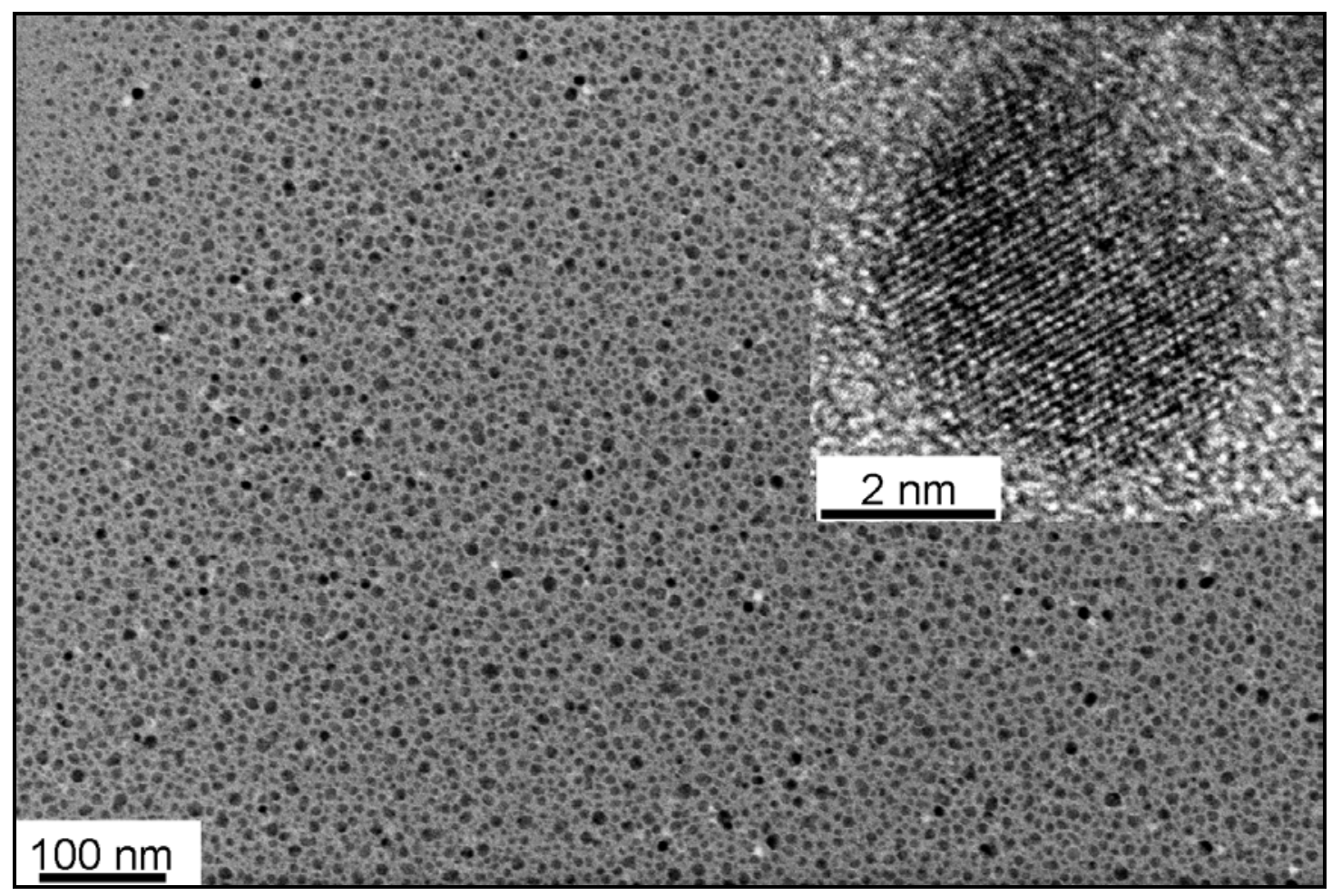

Figure 2-4: TEM micrograph of silicon quantum dots capped with allylamine

\section{2-2. Selected Area Electron Diffraction (SAED)}

\section{2-2-1. The structure factor $F(\theta)$}

The next introductory step in discussing electron scattering is to take the idea of individual atoms scattering electrons (the atomic scattering factor) and consider what happens when the atoms stacked together in crystals. The structure factor $F(\theta)$ is a 
measure of the amplitude scattered by a unit cell of a crystal structure. Because it is amplitude like $f(\theta)$, it also has dimensions of length. $F(\theta)$ can be defined in terms of the sum of the atomic scattering factors from all the $i$ atoms in the unit cell (with atomic coordinates $\left.x_{i}, y_{i}, z_{i}\right)$ multiplied by the phase factor that takes account of the difference in phase between waves scattered from atoms on different planes within the unit cell, described by the Miller indices $(h k l)$. The scattering angle, $\theta$, is the angle between the incident and scattered electron beams. Therefore the $F(\theta)$ can be described as the equation below.

$$
F(\theta)=\sum_{i} f_{i} e^{i \varphi} i=\sum_{i} f_{i} e^{2 \pi i}\left(h x_{i}+k y_{i}+l z_{i}\right)
$$

The equation describes how the amplitude and angle of scattered intensity is influenced by the type of atom and its position of the atom in the cell $(x, y, z)$.

\section{2-2-2. Diffraction Equations}

Von Laue in Germany in 1912 used the well-known light-optics approach to argue that the diffracted waves are in phase if the path difference between waves scattered by adjacent scattering centres is a whole number of wavelengths, $h \lambda$, where $h$ is an integer. Thus as shown in Figure 2-5, if the scattering centres (B and C) are spaced some distance apart, $a$ and the incident beam (wavelength $\lambda$ ) makes an angle $\theta_{1}$ with the line connecting the scattering centres, and is diffracted at an angle $\theta_{2}$, then the path difference (AB-CD) is described in equation 2-5.

$$
a\left(\cos \theta_{1}-\cos \theta_{2}\right)=h \lambda
$$




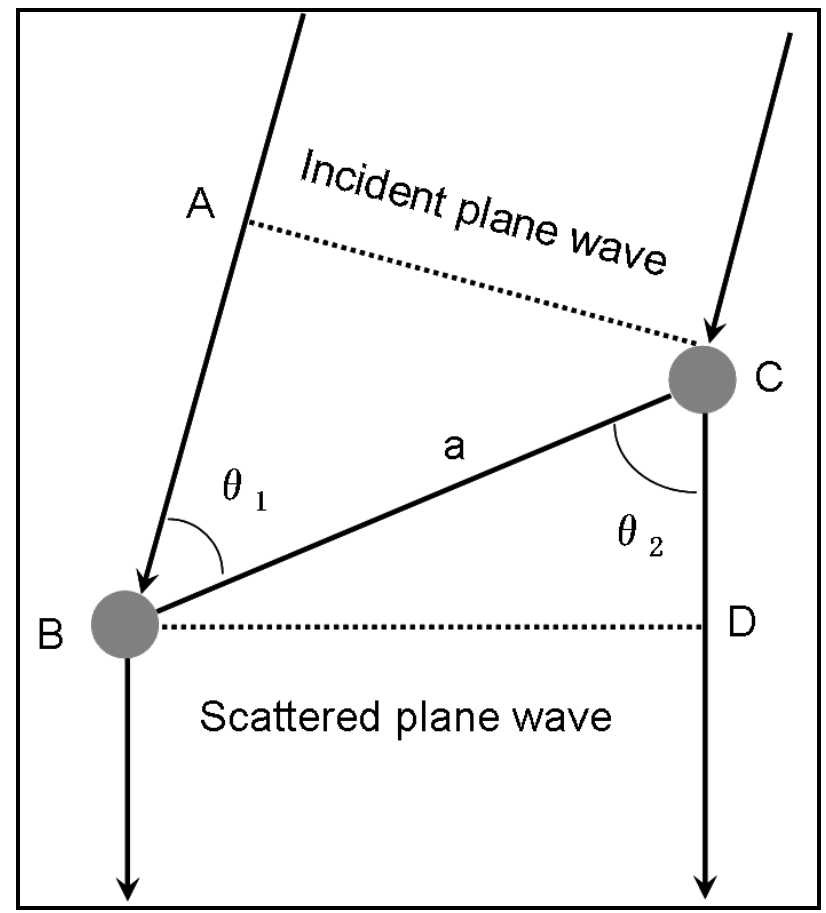

Figure 2-5: The approach used by Von Laue to calculate the path difference for a wave (wavelength $\lambda$ )

Now in three dimensions, two more Laue equations can be written for two more distances, $b$ and $c$, and appropriate angles $\theta_{n}$. (eq. 2-6)

$$
\begin{aligned}
& b\left(\cos \theta_{3}-\cos \theta_{4}\right)=k \lambda \\
& c\left(\cos \theta_{5}-\cos \theta_{6}\right)=l \lambda
\end{aligned}
$$

In a TEM specimen, if all three Laue equations are satisfied simultaneously, a diffracted beam is produced. The letters, $h k l$, are the indices of the diffracted beam and are equivalent to the Miller indices ( $h k l)$ of a crystal plane. This approach of Von Laue was simplified by Bragg in England, who argued that the waves behaved as if they were reflected off atomic planes as shown in Figure 2-6. Bragg showed that waves reflected off adjacent scattering centres must have a path difference equal to an integral number of wavelengths if they are to remain in phase. So in the TEM, the path difference between electron waves reflected from the upper and lower planes in Figure 2-6 is $(\mathrm{AB}+\mathrm{BC})$. Thus if the "reflecting" $(\mathrm{hkl})$ planes are apart with a distance $d$ and the wave is incident and reflected at an angle $\theta_{\mathrm{B}}$, both $\mathrm{AB}$ and $\mathrm{BC}$ are equal to $d \sin \theta_{\mathrm{B}}$ and the total path difference is $2 d \sin \theta_{\mathrm{B}}$. This is called Bragg's law and is shown in equation 2-7. 
$\theta_{\mathrm{B}}$ is reserved for the Bragg angle which is the most important scattering angle in TEM.

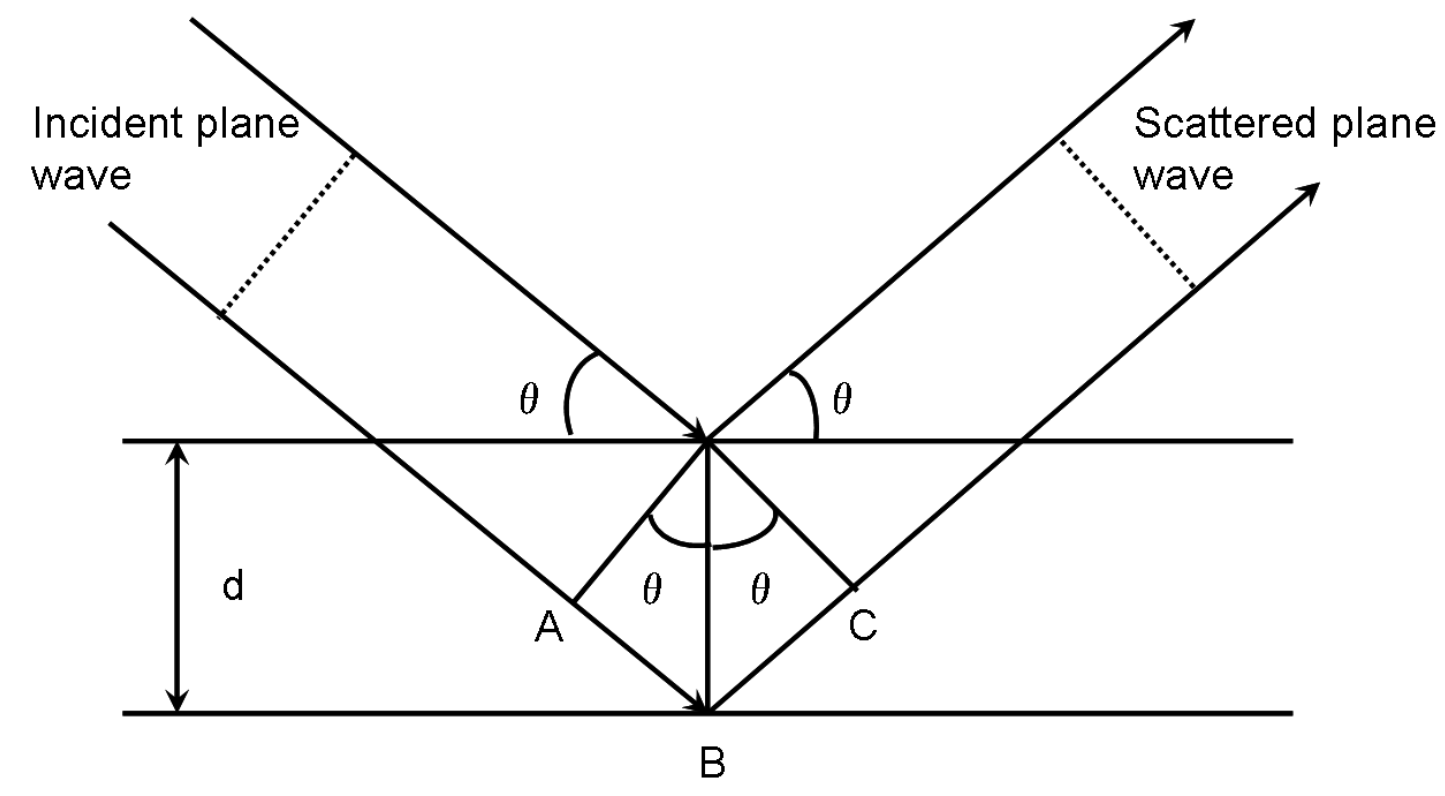

Figure 2-6: The Bragg description of diffraction in terms of the reflections of a plane wave (wavelength $\lambda$ ) incident at an angle $\theta$ to atomic plane of spacing $d$.

\section{2-2-3. Ring patterns from polycrystalline materials}

Diffraction from polycrystalline specimens can be viewed in much the same as X-ray diffraction from powders. For a completely random polycrystal, the rings that are recorded in powder patterns can be seen. If the polycrystal is textured, then there is usually one special plane which is nearly common to all the grains.

The diameter of diffraction rings are proportional to the Bragg angle and the camera length (effective magnification of the electron diffraction pattern), while the intensities of the rings are proportional to the structure factors.

The electron diffraction pattern of silicon is shown in Figure 2-7.

By measuring the diameter and relative intensity of the diffraction rings, it is possible to index, or assign an $h k l$ value, by comparison to the known diameter and intensity ratios of a known crystal structure (ratio method), or by calculating corresponding $d$ 
values for rings by comparison to a diffraction pattern of a standard material (e.g. gold). Indexing of an electron pattern allows confirmation of the crystal structure of the sample.

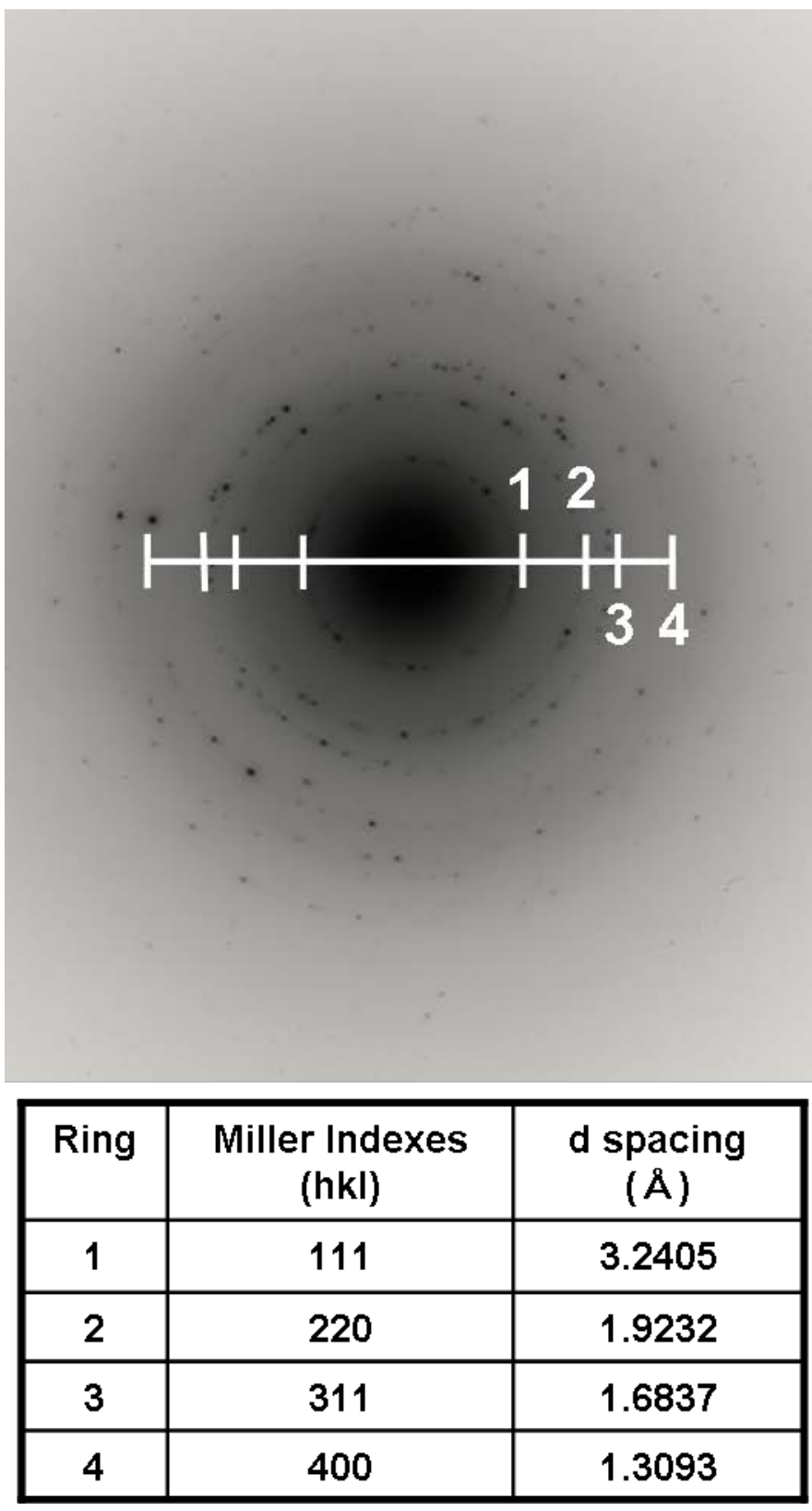

Figure 2-7: Selected area electron diffraction pattern of crystalline silicon 


\section{2-3. Energy Dispersive X-ray Spectrometry (EDS)}

\section{2-3-1. Basic theory}

Inelastically scattered electrons, where electrons lose energy when they hit the specimen, can be used indirectly in the technique EDS, where the characteristic Xrays produced in the specimen are measured.

$\mathrm{X}$-ray emission is the most important secondary signal generated in the specimen. From the emitted X-ray we can find out easily what elements constitute the part of the specimen interacting with the electron beam and we can also quantify the amount of each element in quite straight-forward manner. ${ }^{4,5,6,7}$

\section{2-3-2. Characteristic X-rays}

To produce characteristic X-rays, a high energy beam electrons must penetrate through the outer electron shells and interact with the inner-shell electrons. If more than a critical energy is transferred to an inner-shell electron by a collision with a primary electron, that electron is ejected. Therefore that secondary electron escapes the attractive field of nucleus, leaving a hole in the inner-shell. When this happens the atom is ionised and left in an excited state. The ion can return to a lower energy state by filling in the missing electron with one from outer shells. It is this transition which is accompanied by the emission of an X-ray. The energy of the emitted X-ray photon is characteristic of the difference in energy of the two electron shells involved, and so is characteristic of the element. The mechanism is shown in Figure 2-8.

The difference in the two shell energies equals the energy of the characteristic X-ray. If a K-shell (1s) hole is filled from L-shell ( $2 p$ ) we get a $\mathrm{K}_{\alpha} \mathrm{X}$-ray. If the hole is in the L-shell ( $n=2)$ and is filled from the M-shell $(n=3)$ we get a $L_{\alpha}$ X-ray. 


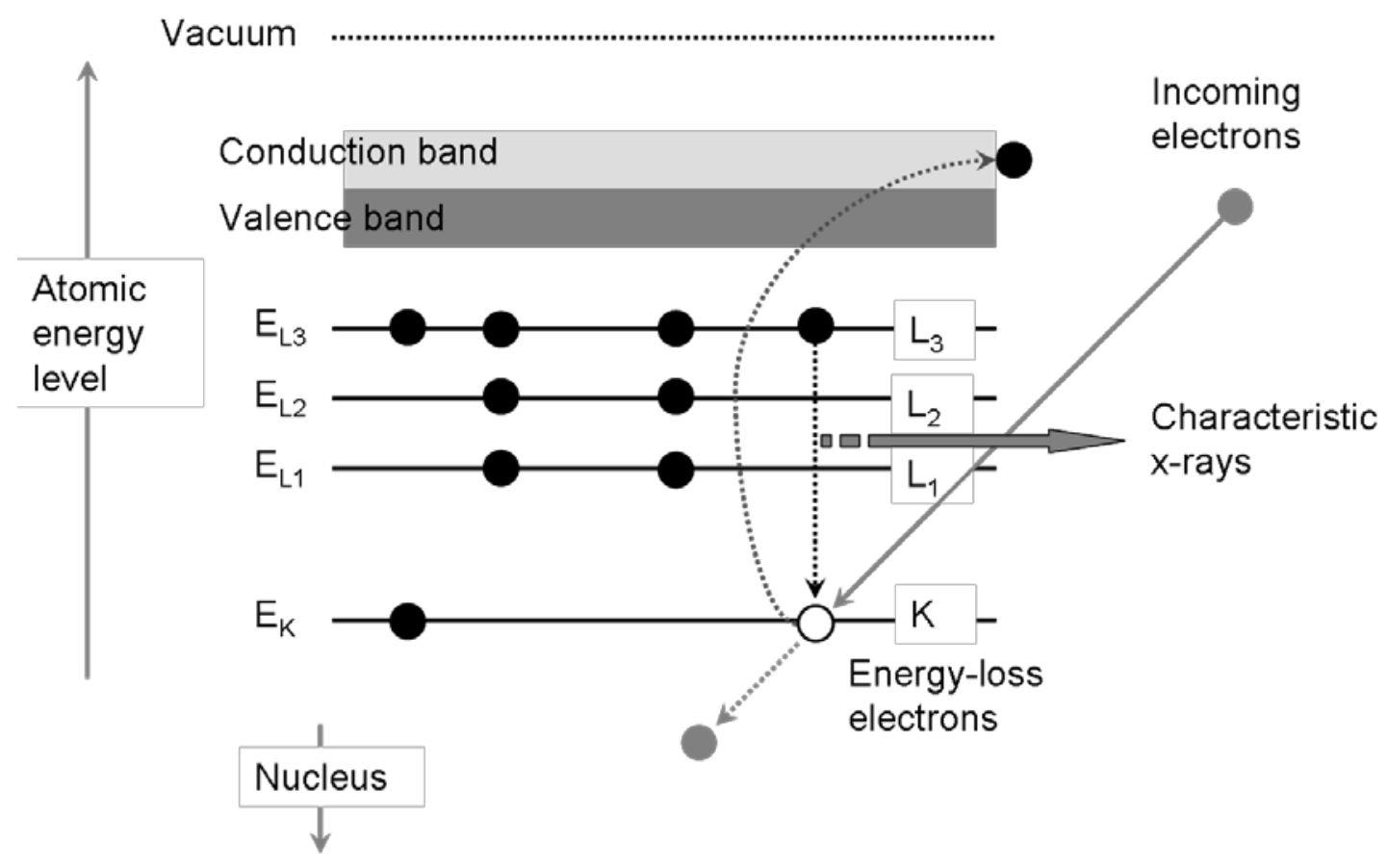

Figure 2-8: The ionisation process

EDS analysis was used to characterise qualitatively the components in the sample by detecting characteristic X-rays. EDS analysis also gives quantitative data of each component in the sample after various corrections have been applied, however it is too difficult to apply this function to silicon quantum dots since EDS detector is made of silicon, absorption of X-rays from the sample can lead to fluorescence within the detector, causing false silicon signal. For qualitative data, obvious differences of the peak of silicon can be seen. When sample contains silicon, the peak of silicon is much larger compared to the silicon peak only from detector. The difference can be seen in Figure 2-9 (a) and (b). 


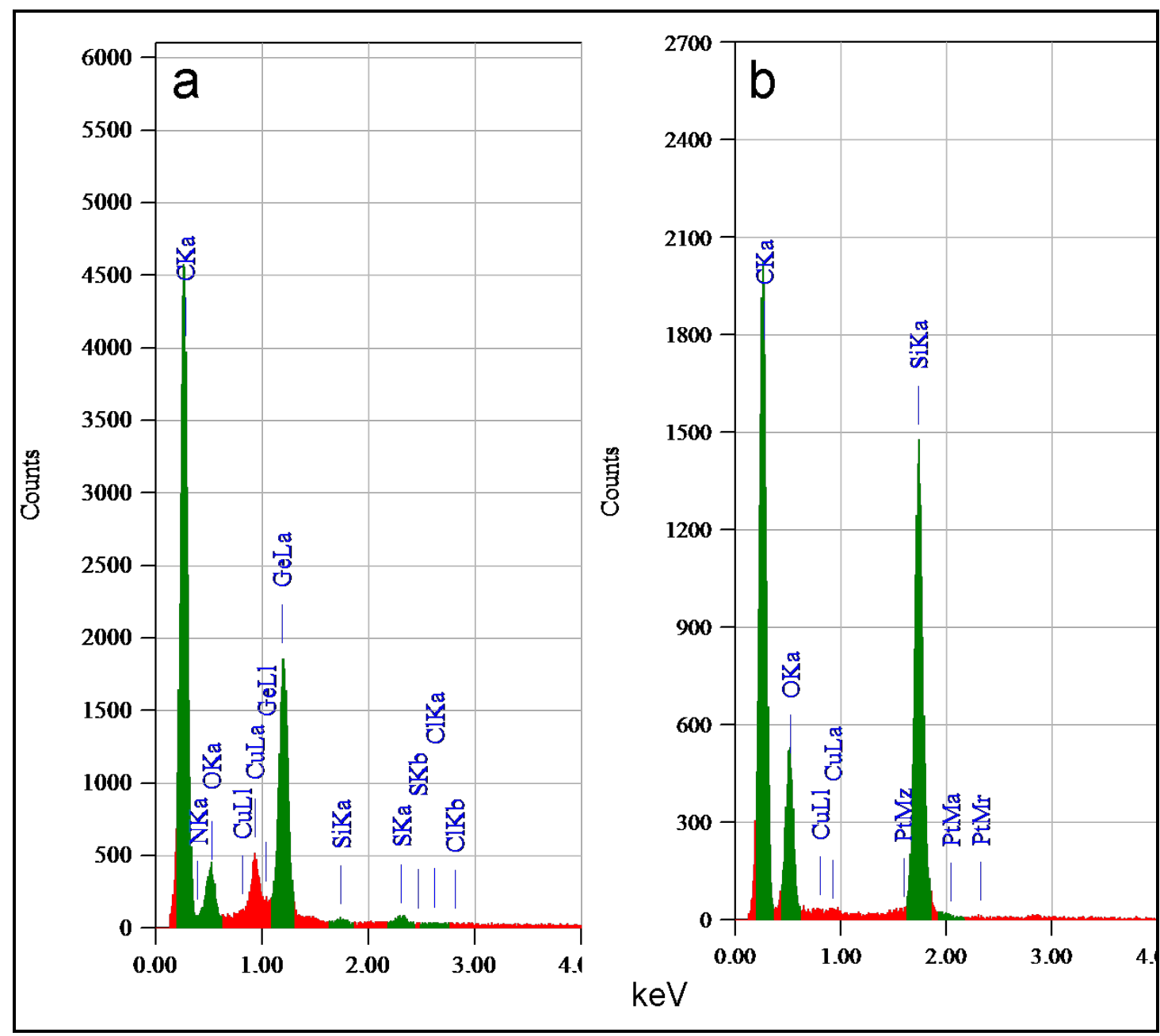

Figure 2-9: (a); EDS spectrum of germanium quantum dots, (b); silicon quantum dots

\section{2-4. Fourier Transform Infrared spectroscopy (FTIR)}

A Perkin-Elmer FT-IR spectrometer was used to identify and characterize the structure of the molecules attached to the nanoparticle surfaces. FTIR sample was prepared as a pellet using potassium bromide $(\mathrm{KBr}) . \mathrm{KBr}$ powder was ground to fine powder in quartz mortar with quartz pestle. A droplet of the sample was mixed with the powder and solvent was dried in an oven for 10 minutes. The mixture powder was placed in a die set and pressed to form a pellet. 


\section{2-4-1. Basic theory}

Infrared spectroscopy is the subset of spectroscopy that deals with infrared region of the electromagnetic spectrum. The most common technique is a form of absorption spectroscopy. FTIR spectroscopy can be used to identify compounds and functional groups, or to investigate changes in chemical bonding. ${ }^{8,9}$

Infrared radiation in the range from about 10,000 to $100 \mathrm{~cm}^{-1}$ is absorbed and converted by a molecule into energy of molecular vibration. Particularly the range between 4000 and $400 \mathrm{~cm}^{-1}$ is commonly measured. The frequency of absorption is dependent on the relative masses of bonded atoms, the force constants of the bonds, and geometry of the atoms. Band intensity can be expressed either as transmittance (T) or absorbance (A). Transmittance is the ratio of the radiant power transmitted by a sample to the radiant power incident on the sample.

Absorbance is the logarithm, to the base 10, of the reciprocal of the transmittance; $A=\log _{10}(1 / T)$.

\section{2-4-2. Molecular vibration}

There are two types of molecular vibrations. One is stretching and the other one is bending. A stretching vibration is movement along the bond axis such that the interatomic distance is increasing or decreasing. A bending vibration may consist of a change of the dihedral angle between bonds which have a common atom in between or the movement of a group of atoms against the remainder of the molecule in which the atoms are considered not moving each other. For example, twisting, rocking, and torsional vibrations involve a change in bond angles in regard to a set of coordinates arbitrarily set up within the molecule. Among those vibrations, only ones that result a rhythmical change in a dipole moment of the molecule are observed in the IR. The typical vibrations are shown below in Figure 2-10.

A molecule has as many degrees of freedom as the total number of degrees of freedom of its individual atoms. Each atom has three degrees of freedom corresponding to the Cartesian coordinates $(x, y, z)$ and it is necessary to describe its position relative to other atoms in the molecule. Therefore a molecule composed of $n$ atoms has $3 n$ degrees of freedom. For non-linear molecules, three degrees of freedom 
describe rotation and three describe translation therefore the remaining 3n-6 degrees of freedom are vibrational degrees of freedom or fundamental vibrations. Linear molecules have $3 n-5$ vibrational degrees of freedom because only two degrees of freedom are needed to describe rotation.

\begin{tabular}{|l|l|l|l|l|l|}
\hline $\begin{array}{l}\text { Symmetrical } \\
\text { stretching }\end{array}$ & $\begin{array}{l}\text { Antisymmetrical } \\
\text { stretching }\end{array}$ & Scissoring & Rocking & Wagging & Twisting \\
\hline & & &
\end{tabular}

Figure 2-10: Representative vibrations of atoms

The theoretical number of fundamental vibrations (absorption frequencies) will hardly be observed because overtones (multiples of a given frequency) and combination tones (sum of two other vibrations) increase the number of bands, however other phenomena reduce the number of bands. Main causes of bands are below.

1. Fundamental frequencies outside of the region of the $4000-400 \mathrm{~cm}-1$.

2. Fundamental bands that are too weak to be observed

3. Fundamental vibrations that are so close that they coalesce

4. A degenerate band from several absorptions which have same frequency in highly symmetrical molecules

5. Fundamental vibrations which do not involve a change in dipole moment of the molecule (and so are not infrared active)

Assignments for stretching frequencies can be approximated by the application of Hooke's law. In the application of the law two atoms and their connecting bond are treated as a simple harmonic oscillator composed of two masses joined by a spring. The following equation derived from Hooke's law states the relationship between frequency of oscillation, atomic masses and the force constant of the bond: (eq. 2-8)

$$
\bar{v}=\frac{1}{2 \pi c}\left[\frac{f}{(M x M y) /(M x+M y)}\right]^{1 / 2}
$$


Where $\bar{v}$ is the vibrational frequency $\left(\mathrm{cm}^{-1}\right)$, c is velocity of light $(\mathrm{cm} / \mathrm{s})$ and $f$ is force constant of bond (dyne/cm). Mx and My is mass (g) of atom $x$ and atom $y$ respectively. The value of $f$ is approximately $5 \times 10^{5}$ dyne/cm for single bonds and approximately two and three times this value for double and triple bonds, respectively.

\section{2-4-3. FTIR spectrum}

By analysing the change in characteristic vibrational frequencies of bonds within the molecule, it is possible to detect changes in bonding, for example related to alteration of functional groups within the molecule due to chemical transformations or due to association between the molecule and the nanocrystal surface. FTIR spectrum of the silicon quantum dots capped with 1-hexene is shown in Figure 2-11.

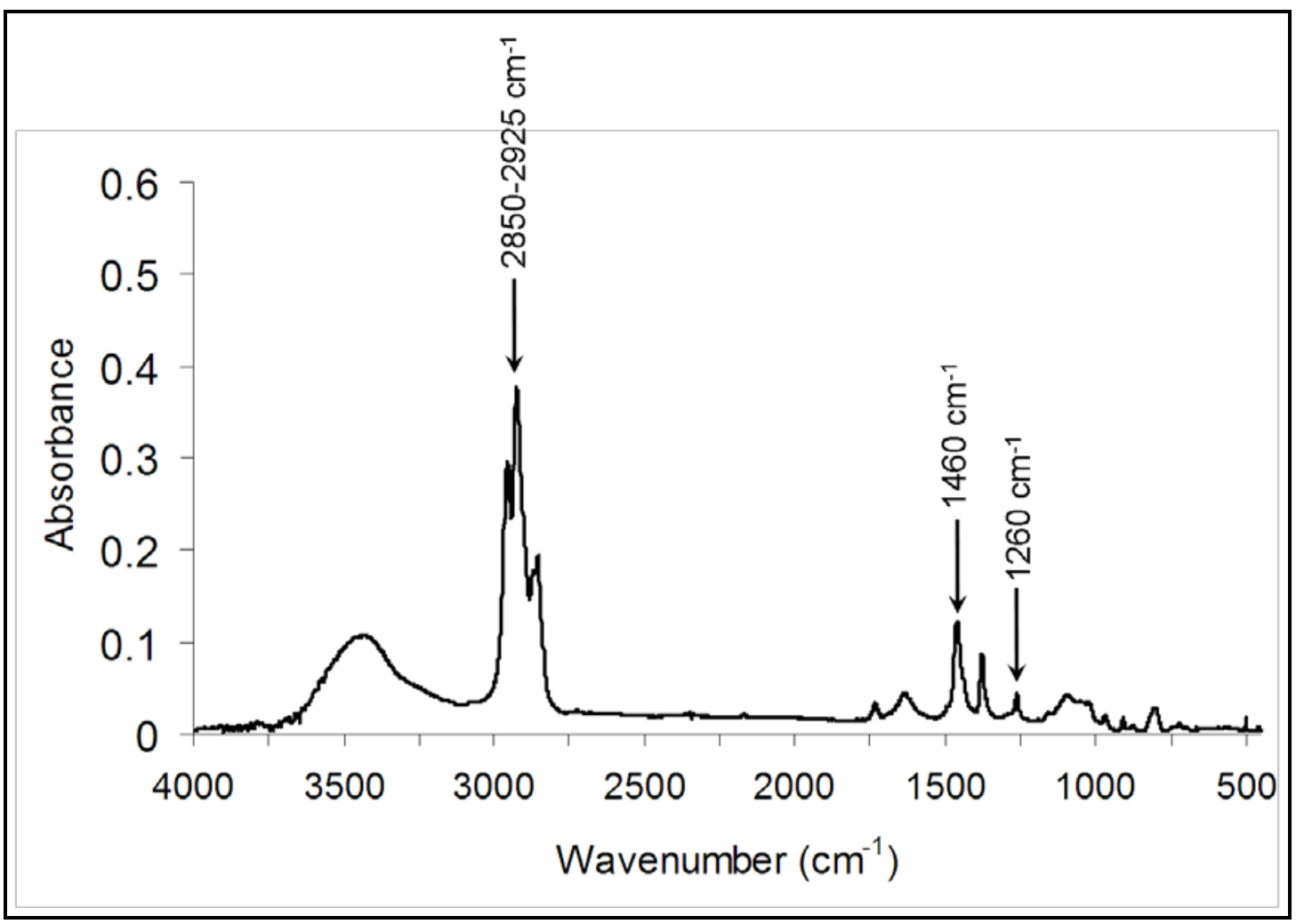

Figure 2-11: FTIR spectrum of the silicon quantum dots capped with 1-hexene

As may be seen in Figure 2-11, the vibrational scissoring and symmetric bending of $\mathrm{Si}-\mathrm{CH}_{2}$ can be seen at $1460 \mathrm{~cm}^{-1}$ and $1260 \mathrm{~cm}^{-1}$. On the other hand, the peak 
corresponding to $\mathrm{C}-\mathrm{H}$ stretching next to the $\mathrm{C}=\mathrm{C}$ bond cannot be seen in this figure which shows the attachment of 1-hexene to the surface of the silicon quantum dot via $\mathrm{C}=\mathrm{C}$ bond.

\section{2-5. Nuclear Magnetic Resonance (NMR)}

\section{Spectroscopy}

${ }^{1} \mathrm{H}$ NMR (500 MHz) spectra of samples in $\mathrm{CDCl}_{3}$ were recorded on a Varian Unity Inova $500 \mathrm{MHz}$ spectrometer. The solvent of each sample solution was removed by evaporation using a rotary evaporator at $60{ }^{\circ} \mathrm{C}$. After removal of the solvent, the sample was freeze-dried overnight and dissolved in $\mathrm{CDCl}_{3}$.

\section{2-5-1. Theory}

Nuclear magnetic resonance is a property that nuclei have in an applied magnetic field, whereby applied electromagnetic (EM) pulses at the resonance frequency stimulate transitions between nuclear magnetic states. Relaxation to the ground state is accompanied by magnetic field fluctuations, which induce a signal. The specific resonance frequency depends on the strength of the applied magnetic field and the nuclide. This allows the observation of specific quantum mechanical magnetic properties of an atomic nucleus. ${ }^{10}$

The total spin angular momentum of the nucleus can be described in terms of total spin quantum number $I$. This number can have values of $0,1 / 2,1,3 / 2$ and so on $(I=0$ means no net spin angular momentum). The spin number $I$ can be determined from the mass number and the atomic number. Some nuclides such as ${ }_{1}^{1} \mathrm{H},{ }_{1}^{3} \mathrm{H},{ }_{6}^{13} \mathrm{C}$ have total spin quantum number $I$ of $1 / 2$ and they have uniform spherical charge, therefore their spectra can be obtained more easily. Among those nuclides, ${ }^{1} \mathrm{H}$ and ${ }^{13} \mathrm{C}$ are the most widely used for NMR spectroscopy. The nuclides which have spin number(I) larger than 1 do not have spherical charge distribution and it complicates spectroscopy. Within a sample in an applied magnetic field there is a slight excess of nuclei population in the lower energy state $\left(\mathrm{N}_{\alpha}>\mathrm{N}_{\beta}\right)$. The net imbalance in the populations of the energy states leads to the sample possessing a net magnetic moment, which processes around the axis of the applied magnetic field ( $z$ axis) at the Larmour frequency of the nuclide (see schematic diagram in Figure 2-12). These energy states 
are described as $1 / 2$ and $-1 / 2$ and difference in energy between them, $\Delta \mathrm{E}$, is given by the equation below: (eq. 2-9)

$$
\Delta E=\frac{h \gamma}{2 \pi} B_{0}
$$

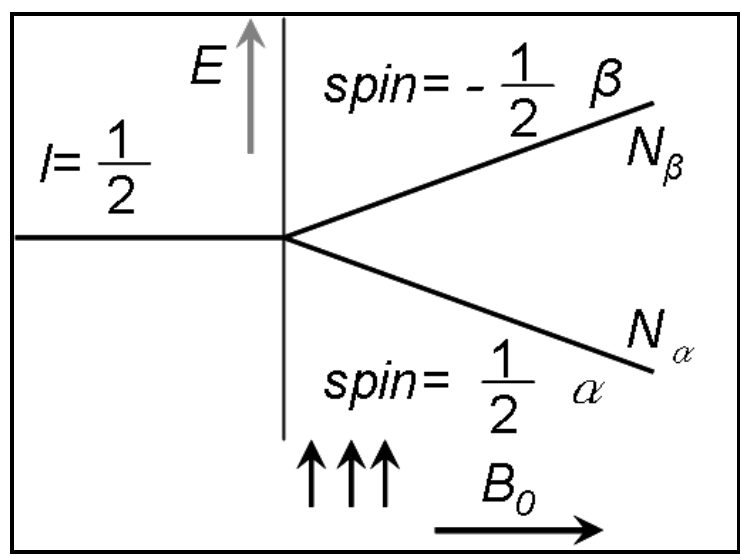

Figure 2-12: Two nuclear magnetic energy levels in a magnetic field of magnitude $B_{0}$. $\mathrm{N}$ is population. The direction of the magnetic field is up.

Where $h$ is Plank's constant, $\gamma$ is the gyromagnetic ratio, and $B_{0}$ is the strength of the applied magnetic field. This states that $\Delta \mathrm{E}$ is proportional to $\mathrm{B}_{0}$ since $h, \gamma$ and $\pi$ are constants. The constant $\gamma$ is called the gyromagnetic ratio. It is a fundamental nuclear constant and it is the proportionality constant between the magnetic moment $\mu$ and the spin number, $I$. (eq. 2-10)

$$
\gamma=\frac{2 \pi \mu}{h I}
$$

Once two energy levels for the nuclei in the sample have been established, it is possible to introduce energy in the form of radiofrequency radiation $\left(v_{1}\right)$ to effect a transition between these energy levels in a stationary magnetic field of given strength $\mathrm{B}_{0}$. The fundamental NMR equation correlating the applied radiofrequency $v_{1}$ with the magnetic field strength is: (eq. 2-11)

$$
v_{1}=\frac{\gamma}{2 \pi} B_{0}
$$

since $\Delta E=h v$ 
The introduced radiofrequency $v_{1}$ is given in megahertz $(\mathrm{MHz})$. A frequency of 100 $\mathrm{MHz}$ is needed at a magnetic field strength $\left(\mathrm{B}_{0}\right)$ of 2.35 tesla(T) for the proton NMR. At this frequency the system is in resonance. Energy is absorbed by the nucleus, raising it to the higher energy state, and displacing the net magnetisation from the $z$ axis. When the population of nuclei relaxes to the ground state, the return of the processing net magnetisation to the $z$ axis induces a signal in the detector coils of the NMR spectrometer in the form of an oscillating voltage (Free Induction Decay, FID). Fourier transform yields spectrum of the frequency components of the FID, which correspond to resonance frequencies of nuclides in sample.

The radiofrequency $v_{1}$ can be introduced either by continuous wave scanning or by a radiofrequency pulse.

\section{2-5-2. Chemical shift}

In accordance with the basic NMR equation above (2-10), only a single proton peak should be observed for a nucleus (e.g. proton) in a uniform applied magnetic field. However, the reality is not that simple. A nucleus (proton) in a molecule is surrounded by electron cloud. When the external magnetic field is applied to the molecule the electron cloud generates a very small local magnetic field. This local magnetic field orients the opposite direction from the external magnetic field therefore the effective magnetic field which can be applied to the proton is slightly smaller than the external magnetic field. This effect of the local magnetic field by the electron cloud is called "shielding". The electronic environment of each nucleus (proton) in the molecule is slightly different from each other, which changes the degree of shielding of each nucleus (proton). Therefore the effective magnetic field which is applied to each nucleus (proton) is different, and in accordance with the basic NMR equation (2-11) the frequency differs. Very rough approximation, the degree of shielding of a nucleus such as a proton on a carbon atom will depend on the inductive effect of other groups attached to the carbon atom. The difference in the absorption position of a particular proton from the absorption position of a reference proton is called "chemical shift" of the particular proton, and can be used to identify particular nuclei within the molecule and monitor for changes in the chemical environment. 


\section{2-5-3. Spin coupling}

Spin coupling can be described as the indirect coupling of nuclear (proton) spins through the intervening bonding electrons. Very briefly, it occurs because there is some tendency for a bonding electron to pair its spin with the spin of the nearest nucleus (proton). The spin of a bonding electron having been thus influenced, the electron will affect the spin of the other bonding electron, and so on, through to the adjacent nuclei (protons). Coupling is usually not important beyond three bonds unless there is ring strain as in small rings or bridged systems, delocalisation as in aromatic or unsaturated systems, or four connecting bonds in a W configuration. Twobond coupling is termed "geminal" and three-bond coupling is termed "vicinal".

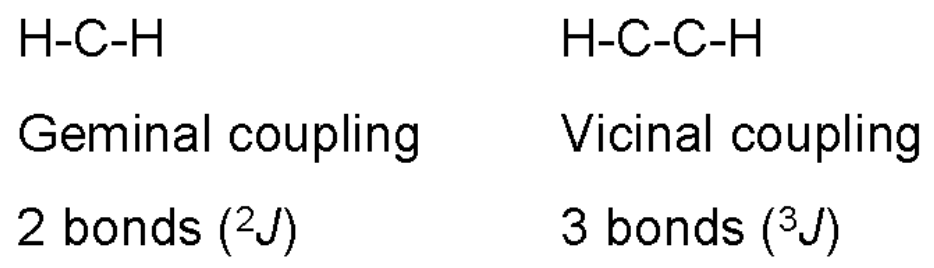

In the molecule below, the chemical environment of two vicinal protons are quite different from one another (due to inductive effects).

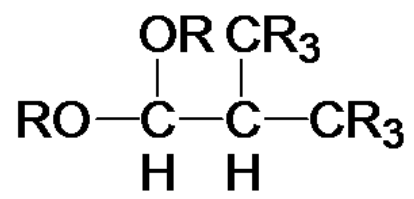

Each proton will show absorption and the absorption will be widely separated in frequency, but the spin of each proton is slightly affected by the two possible orientations of the spin of the other proton, through the intervening electrons. Because of the reason above at the two energy states exist for each proton spin - aligned with or against the spin of the vicinal proton, each absorption appears as a doublet (two peaks). The frequency difference in $\mathrm{Hz}$ between the component peaks of a doublet is proportional to the effectiveness of the coupling and is denoted by a coupling constant, $J$, which is independent of the external magnetic field $\mathrm{B}_{0}$.

So long as the chemical shift difference in hertz $(\Delta v)$ is much larger than the coupling constant, the simple pattern of two doublets appears. As $\Delta v / J$ become smaller, the doublets approach one another. The inner two peaks increase in intensity and the outer 
two peaks decrease. The shift position of each proton is no longer midway between its two peaks but is at the "centre of gravity".

\section{2-5-4. ${ }^{1} \mathrm{H}$ NMR spectrum}

The characteristic chemical shift and coupling patterns allow identification of molecular structure. Alternations in chemical bonding can be detected by analysing NMR spectra. ${ }^{1} \mathrm{H}$ NMR spectra of free 1-hexene and the silicon quantum dots capped with 1-hexene are shown in Figure 2-13. 

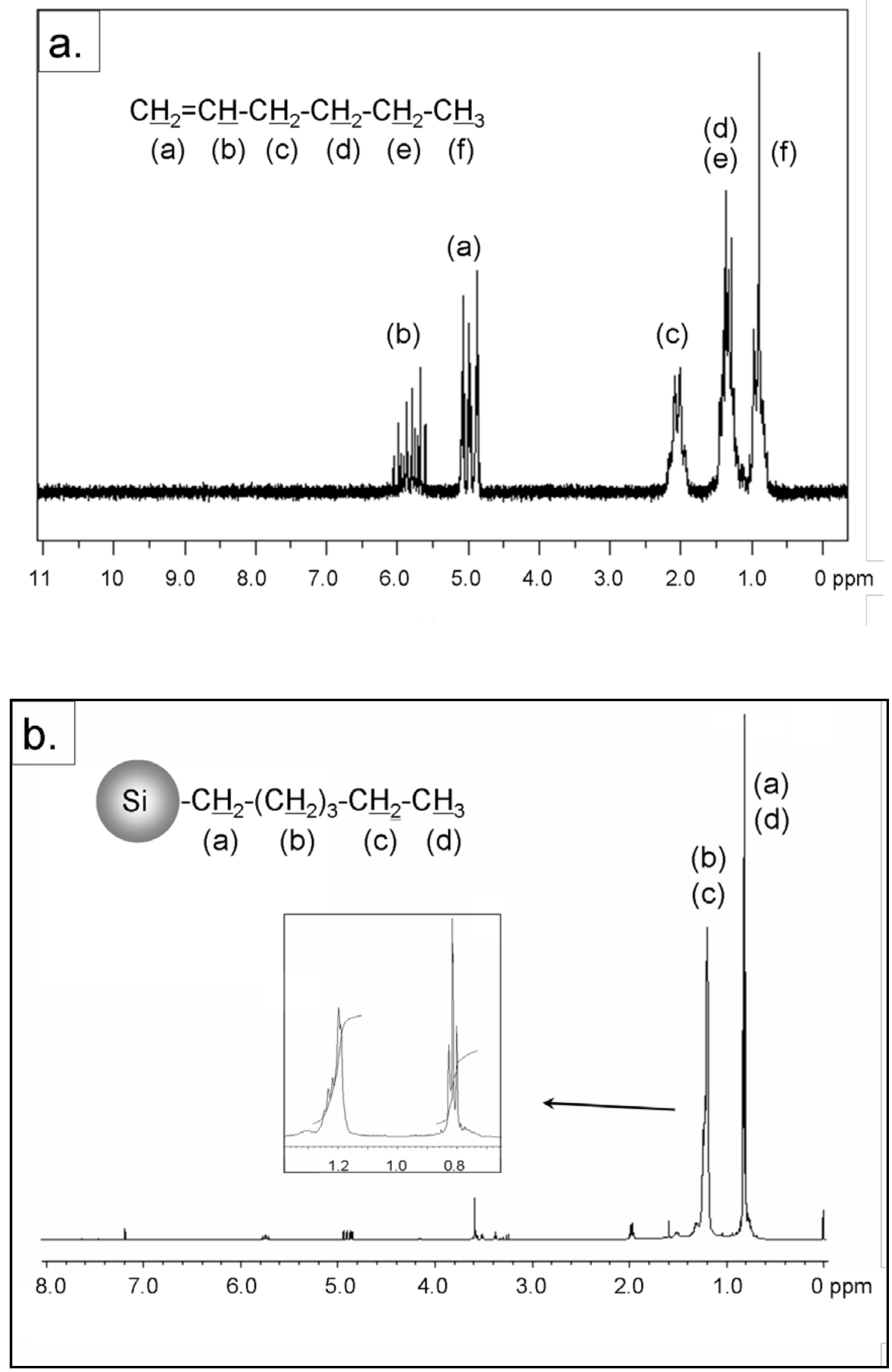

Figure 2-13: ${ }^{1} \mathrm{H}$ NMR spectra of (a); free 1-hexene and (b); the silicon quantum dots capped with 1-hexene 
After the reaction, the signals at $5.80 \mathrm{ppm}, 4.96 \mathrm{ppm}$ and $4.92 \mathrm{ppm}$ which correspond to the terminal double bond of free 1-hexene disappeared. Also the signal at $2.06 \mathrm{ppm}$ from the NMR spectrum of free 1-hexene has disappeared in the NMR of the sample. The triplet at $0.814 \mathrm{ppm}$ integrates for 5 protons, indicating the terminal methyl group and $\mathrm{CH}_{2}$ next to silicon. Usually the methylene signal appears further down field, around $1.0 \mathrm{ppm}$, however by being attached to silicon quantum dot these protons are probably more shielded. The signal observed at $1.192 \mathrm{ppm}$ integrates for the 8 protons attached to the carbons between the methyl group and $\mathrm{Si}-\mathrm{CH}_{2}$.

\section{2-6. UV-VIS Absorption}

Diluted solutions of the samples in organic solvent were put in a $1 \mathrm{~cm}$ width four sided quartz cuvette for UV-VIS absorption measurement. The instrument used was a CARY 100 Scan UV-Visible spectrophotometer operating in a double beam mode. The measurements were carried out with a data interval of $1 \mathrm{~nm}$ and a scan rate of 200 nm per minutes.

\section{2-7. Photoluminescence spectroscopy (PL)}

Diluted solutions of the samples in organic solvent were used for photoluminescence measurement. The same quartz cuvette was used. The studies were performed using a HORIBA Jobin Yvon fluorolog spectrometer. For the silicon quantum dots measurement, $260 \mathrm{~nm}$ to $420 \mathrm{~nm}$ in $20 \mathrm{~nm}$ intervals were used to excite the samples with the slit width of 10 in both sides. For silica nanoparticles, $300 \mathrm{~nm}$ to $500 \mathrm{~nm}$ in $20 \mathrm{~nm}$ intervals were used as excitation wavelengths with the slit of 10 in both sides.

\section{2-8. Cell viability assay (MTT assay)}

The MTT assay is a standard colorimetric assay for measuring cell viability by measuring the activity of enzymes that reduce a substrate to formazan, giving a orange colour. ${ }^{11}$ This method was used to determine cyto-toxicity of silicon quantum dots. 
After the solvent was removed by evaporation, the silicon quantum dots were redispersed in medium (DMEM, Dulbecco’s Modified Eagles Medium) and different concentration solutions were made. These solutions were cultured with the cells in 96well plate.

The cells were co-cultured with the silicon quantum dots and the yellow MTT (3-(4,5Dimethylthiazol-2-yl)-2,5-diphenyltetrazolium bromide, a tetrazole) was added to the well plate. If the cells are alive this substance is reduced to orange formazan in mitochondria. Formazan has absorption around $460 \mathrm{~nm}$ to $480 \mathrm{~nm}$ therefore the cell viability can be quantified by measuring the absorbance of formazan at $480 \mathrm{~nm}$ with the Cary $100 \mathrm{UV}$-vis spectrophotometer.

Human skin fibroblasts (WS1), epithelial cells (A549), and hepatoma cell line (HepG2) were used for this research. 


\section{2-9. References}

1. D. B. Williams, and C. B. Carter; Transmission Electron Microscopy Basics1, Plenum Press, New York, United States of America, 1996.

2. D. B. Williams, and C. B. Carter; Transmission Electron Microscopy Basics2, Plenum Press, New York, United States of America, 1996.

3. (a) R. D. Tilley, PhD Thesis, Department of Chemistry, Cambridge University, 2001.

(b) http://www.uga.edu/ caur/teaching.htm

4. L. Reimer; Transmission Electron Microscopy, Springer-Verlag, Berlin, Germany, 1984.

5. P. Buseck, J. Cowley, and L. Eyring; High-Resolution Transmission Electron Microscopy, Oxford University Press, Oxford, United Kingdom, 1988.

6. T. H. Lim, PhD Thesis, Department of Chemical and Physical Sicences, Victoria University of Wellington, 2008.

7. J. Watt, PhD Thesis, Department of Chemical and Physical Sicences, Victoria University of Wellington, 2009.

8. http://en.wikipedia.org/wiki/Infrared_spectroscopy

9. R. M. Silverstein, and F. X. Webster; Spectrometric Identification of Organic Compounds, John Wiley \& Sons, Inc., New York, United States of America, 1996.

10. J. E. McMurry; Organic Chemistry, Thomson Leaning Inc., New York, United States of America, 2004. 
11. http://en.wikipedia.org/wiki/MTT_assay 


\section{Chapter 3 Synthesis and surface modification of silicon quantum dots}

This chapter describes the synthesis of silicon quantum dots and their subsequent development of surface modification for biological applications. A microemulsion method was utilised to produce hydrogen terminated silicon quantum dots. These hydrogen terminated silicon quantum dots can react with terminal alkenes to form SiC bonds which can prevent them from being oxidised. Since silicon is an indirect band gap semiconductor material, usually it does not luminescence. However, when quantum confinement occurs in silicon nanocrystals below $5 \mathrm{~nm}$ diameter, it starts luminescing. Fluorescent silicon quantum dots have great potential for various applications such as drug delivery system or solar cells.

To control the size of silicon quantum dots many synthesis methods have been reported. As described in the introduction chapter, alkyl terminated silicon quantum dots were synthesised at super critical conditions by Korgel et. al. ${ }^{1-5}$ Mitchell and coworkers synthesised silicon quantum dots by milling bulk silicon in the reactor followed by surface modification with alkenes. ${ }^{6,7}$ However there are still some problems of those synthesis methods regarding functionalisation of the silicon quantum dots or size distributions. In contrast, well functionalised and mono dispersed silicon quantum dots were synthesised by Tilley et. $\mathrm{al}^{8-10}$ using a microemulsion system. Kauzlarich and co-workers also synthesised silicon quantum dots by a solution phase route. ${ }^{11-19}$

Similarly there are many methods for surface modification of quantum dots. However the majority are focused on quantum dots of groups II/VI such as cadmium selenide quantum dots ${ }^{20-22}$ with little research on the surface modification of silicon quantum dots.

In this research we focuses on solution phase synthesis using the microemulsion system which has an advantage of providing smaller size distribution. ${ }^{23,24}$ Also this synthesis is conducted at room temperature which provides wider options for capping agents. In this synthesis system, silicon halide such as silicon tetrachloride or silicon tetrabromide was used as a precursor and it was trapped in the reverse micelle inside 
the organic solvent. After the solution was homogenised, the precursor was reduced by hydride agent such as lithium aluminium hydride or lithium borohydride to form hydrogen terminated silicon quantum dot. The surface of this hydrogen terminated silicon quantum dot is passivated with capping agent (alkenes) to prevent from oxidation by using platinum catalyst or UV-irradiation $(254 \mathrm{~nm})$. The reaction scheme of the synthesis is shown in Scheme 3-1. This chapter focused on the surface modification of silicon quantum dots by a single step method using various terminal alkene molecules to functionalise the surface. Also two different methods of purification were explored which will be described later in this chapter. Samples were characterised by TEM to assess the size distribution. Surface molecules were characterised by FTIR and NMR to assess the attachment of capping molecules onto the surface of silicon quantum dots.

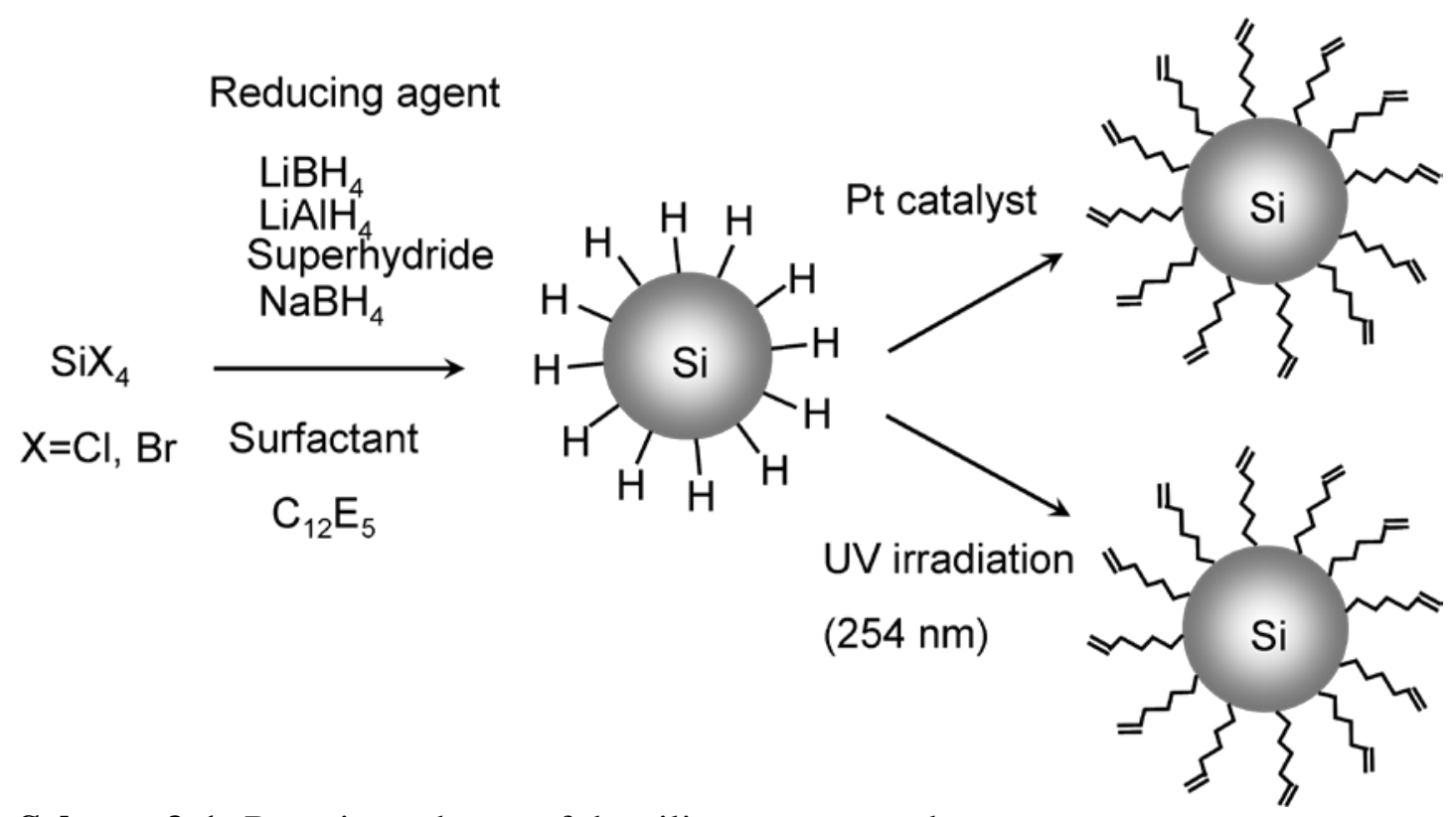

Scheme 3-1: Reaction scheme of the silicon quantum dot 


\section{3-1. Surface modification in single step}

\section{3-1-1. Silicon quantum dots capped with allylalcohol: Experiment 1}

Synthesis of silicon quantum dots capped with allylalcohol was conducted to produce hydroxyl group terminated silicon quantum dots. The terminal double bond of allylalcohol was expected to form a covalent bond with silicon atoms on the surface of the quantum dots and leave the terminal hydroxyl group as a functional group. The hydroxyl group would broaden the possibilities of attaching bio-friendly molecules onto the surface of silicon quantum dots.
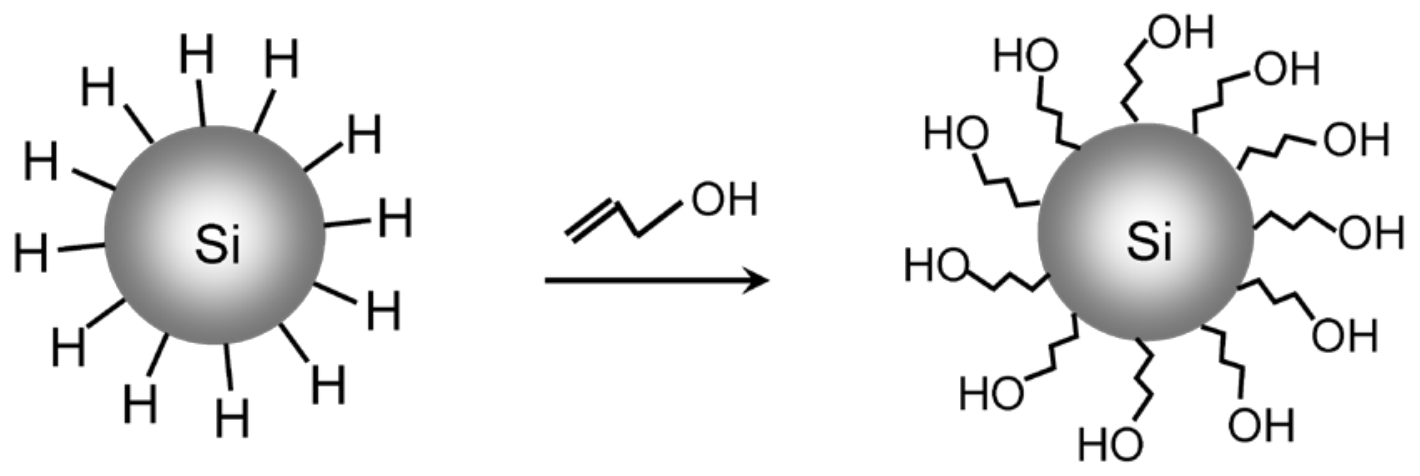

Scheme 3-1-1: Reaction scheme for allylalcohol capping of the silicon quantum dot

\section{General experimental procedure}

All the syntheses of silicon quantum dots were conducted under nitrogen atmosphere.

The surfactant pentaethylene glycol monododecyl ether $\left(\mathrm{C}_{12} \mathrm{E}_{5}\right)$ / tetraoctylammonium bromide (TOAB) was dissolved in $50 \mathrm{~mL}$ of hexane and silicon tetrachloride $\left(\mathrm{SiCl}_{4}\right) /$ silicon tetrabromide $\left(\mathrm{SiBr}_{4}\right)$ was added as a precursor. The solution was stirred for an hour to homogenise and form reverse micelles. Lithium aluminiumhydride /lithium triethylborohydride (superhydride) / lithium borohydride was injected into the solution as a reducing agent to form hydrogen terminated silicon quantum dots. The solution turned cloudy probably due to polar impurities forming colloids. The solution was stirred over night and the white precipitate was removed by filtration. 
For platinum catalyst capping, $0.1 \mathrm{~mL}$ of hexachloro platinic acid / platinum norbornene and capping agent were injected to the reaction mixture and stirred for over night at $60^{\circ} \mathrm{C}$.

For UV-irradiation capping, The reaction mixture was transferred to a UV-reactor vessel and $4.0 \mathrm{~mL}$ of 1,5-hexadiene was added. The reaction mixture was exposed to UV-light (Rayonet RPR 200/ $254 \mathrm{~nm})^{25-30}$ for 5 hours before it was exposed to the air.

In this experiment $0.25 \mathrm{~g}(0.00062 \mathrm{~mol})$ of pentaethylele glycol monododecyl ether $\left(\mathrm{C}_{12} \mathrm{E}_{5}\right), 0.3 \mathrm{~mL}(0.0026 \mathrm{~mol})$ of silicon tetrachloride $\left(\mathrm{SiCl}_{4}\right), 4 \mathrm{~mL}$ of $2.0 \mathrm{M}$ lithium borohydride was used. The surface passivation was conducted with $5 \mathrm{~mL}$ of allylalcohol using hexachloro platinic acid. After the reaction the solution was transferred into a separation funnel and $20 \mathrm{~mL}$ of methanol was added. After three times of liquid-phase separation, the silicon quantum dots were extracted into methanol layer.

\section{Results}

After purification of the sample, the solution did not show blue luminescence as it is supposed to. No nanoparticles were observed from TEM analysis. EDS analysis showed no silicon peak and SAED could not be obtained. The result showed no silicon quantum dots were produced. A possible reason for the failure is due to the terminal hydroxyl group. It might have formed a Si-O covalent bond through the oxygen atom of the hydroxyl group and the double bond from the other terminal was left on the surface as hydrophobic. The methanol layer was collected from the liquidphase separation for the further purification. However no blue luminescence was observed. This results prompted the hypothesis of forming Si-O bond with the hydroxyl group and left the hydrophobic $\mathrm{C}=\mathrm{C}$ double bond at the terminal end.

\section{3-1-2. Silicon quantum dots capped with 1-hexene: Experiment 2, 3}

Silicon quantum dots capped with 1-hexene were synthesized to confirm the success of the chosen synthesis technique and the surface modification of silicon-carbon bond. 
1-hexene was chosen for the surface capping molecules because of its simple structure which can reduce the risk to produce side products and the presence of a terminal double bond to make a covalent bond with silicon. Crystalline nanoparticles were observed from TEM images and the existence of silicon was confirmed by the EDS spectrum from both samples. The surface molecules of the quantum dot from experiment 2 were characterised by NMR and FTIR.
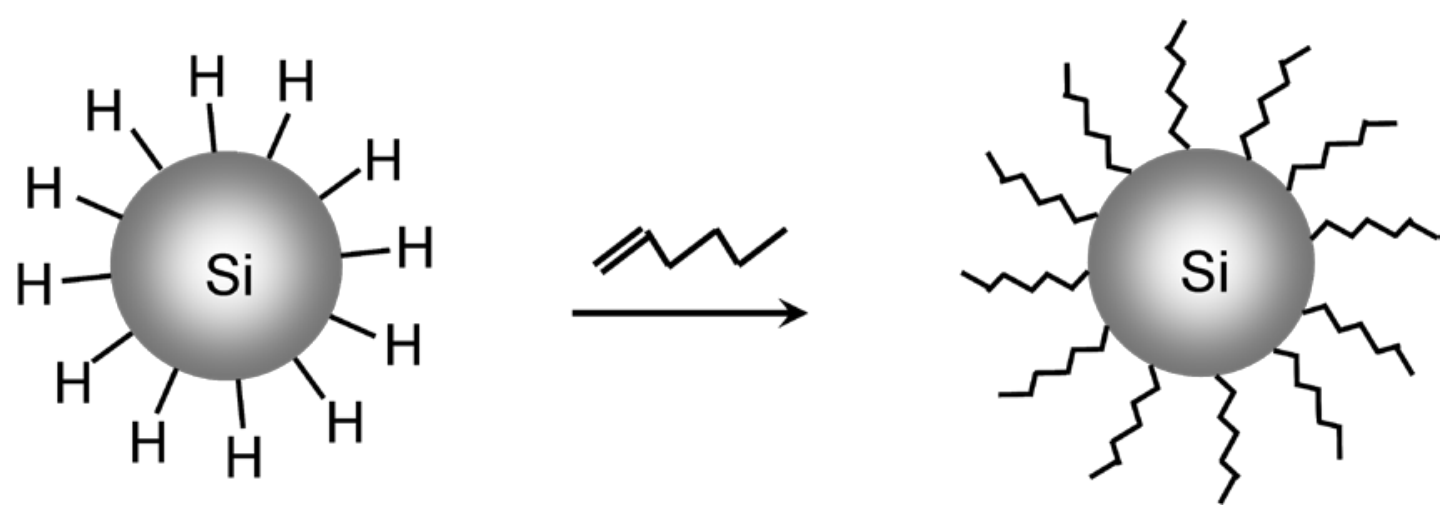

Scheme 3-1-2: Reaction scheme for 1-hexene capping of the silicon quantum dot

\section{Experimental (Experiment 2)}

$1.0 \mathrm{~g}$ of tetraoctylammonium bromide (TOAB) as surfactant, $0.3 \mathrm{~mL}(0.002 \mathrm{~mol})$ of silicon tetrabromide $\left(\mathrm{SiBr}_{4}\right), 3 \mathrm{~mL}$ of $2.0 \mathrm{M}$ lithium triethylborohydride (superhydride) were used in this experiment. After adding the reducing agent, anhydrous methanol $(2 \mathrm{~mL})$ was added to quench any remaining superhydride. The surface passivation was conducted with $5 \mathrm{~mL}$ of anhydrous 1-hexene using hexachloro platinic acid. After the surface passivation, N-methylformamide was added to the reaction mixture in a separation funnel and silicon quantum dots were extracted by liquid-phase separation. The hexane layer was collected and dried under vacuum then the yellow oil was re-dispersed in anhydrous hexane.

\section{Results}

Figure 3-1shows a TEM micrograph of the nanoparticles observed from experiment 1. From high resolution TEM micrograph, lattice fringes were seen, however because of the small sizes, no SAED was obtained. Average size of the nanoparticles was $3.4 \pm$ $0.3 \mathrm{~nm}$. EDS analysis showed that the nanoparticles were mainly composed of silicon (data not shown). 


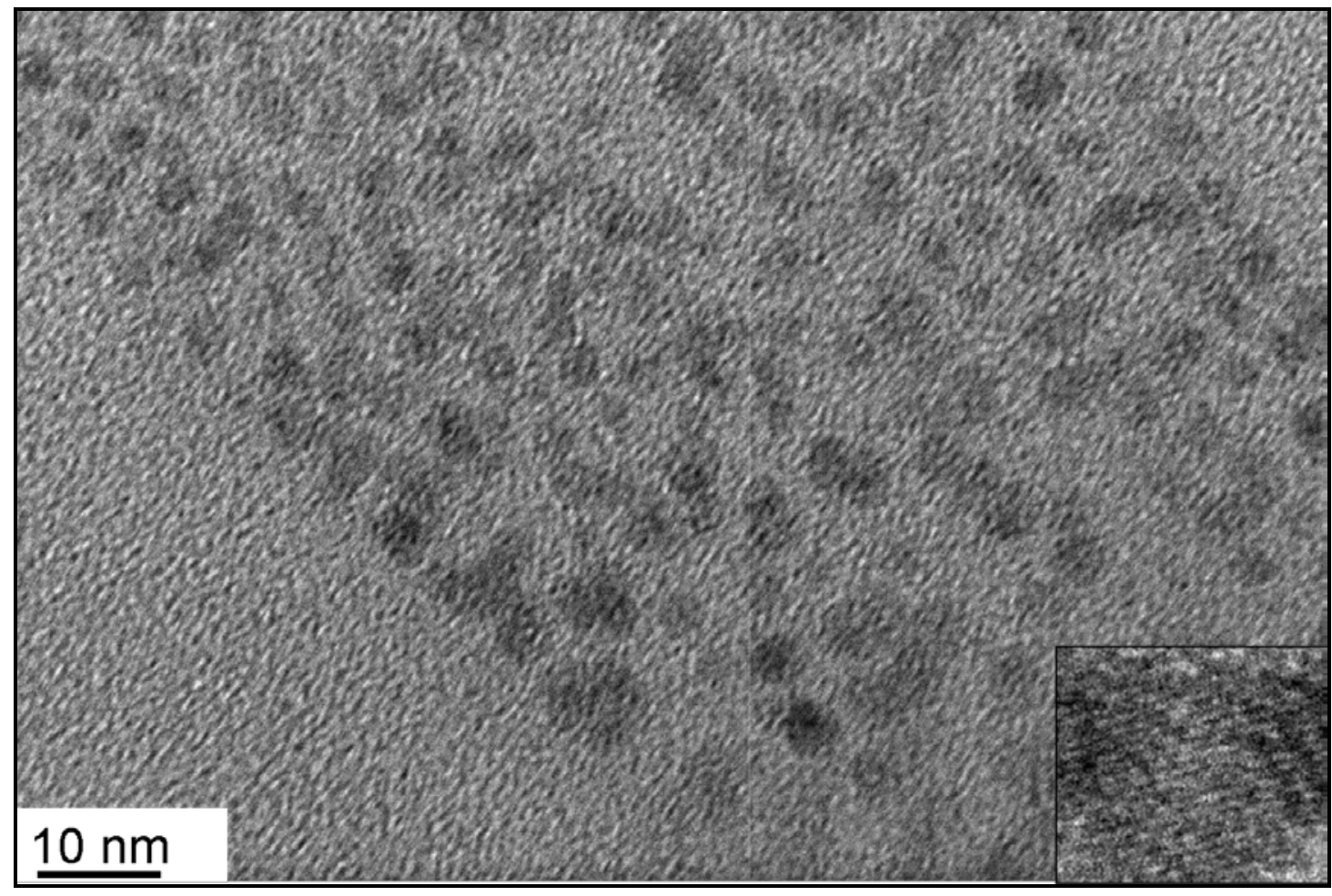

Figure 3-1: TEM micrograph of nanoparticles observed from experiment 2. Inset shows a high resolution image of the sample.

\section{Experimental (Experiment 3)}

In this experiment $0.77 \mathrm{~g}$ of pentaethyleneglycol monododecylether $\left(\mathrm{C}_{12} \mathrm{E}_{5}\right), 0.3 \mathrm{~mL}$ $(0.002 \mathrm{~mol})$ of silicon tetrabromide $(\mathrm{SiBr} 4), 3 \mathrm{~mL}$ of $2.0 \mathrm{M}$ lithium triethylborohydride (superhydride) were used. Surface pssivation was conducted with $5 \mathrm{~mL}$ of anhydrous 1-hexene using hexachloro platinic acid. After the reaction $\mathrm{N}$ methylformamide was added to the reaction mixture in a separation funnel and the silicon quantum dots were extracted by a liquicd-phase separation. The hexane layer was collected and dried under vacuum then the yellow oil was redispersed in anhydrous hexane. 


\section{Results}

As may be seen in Figure 3-2, the crystals were nearly spherical and partially aggregated. The nanoparticles were relatively mono disperse with the average diameter of $4.7 \pm 0.9 \mathrm{~nm}$.

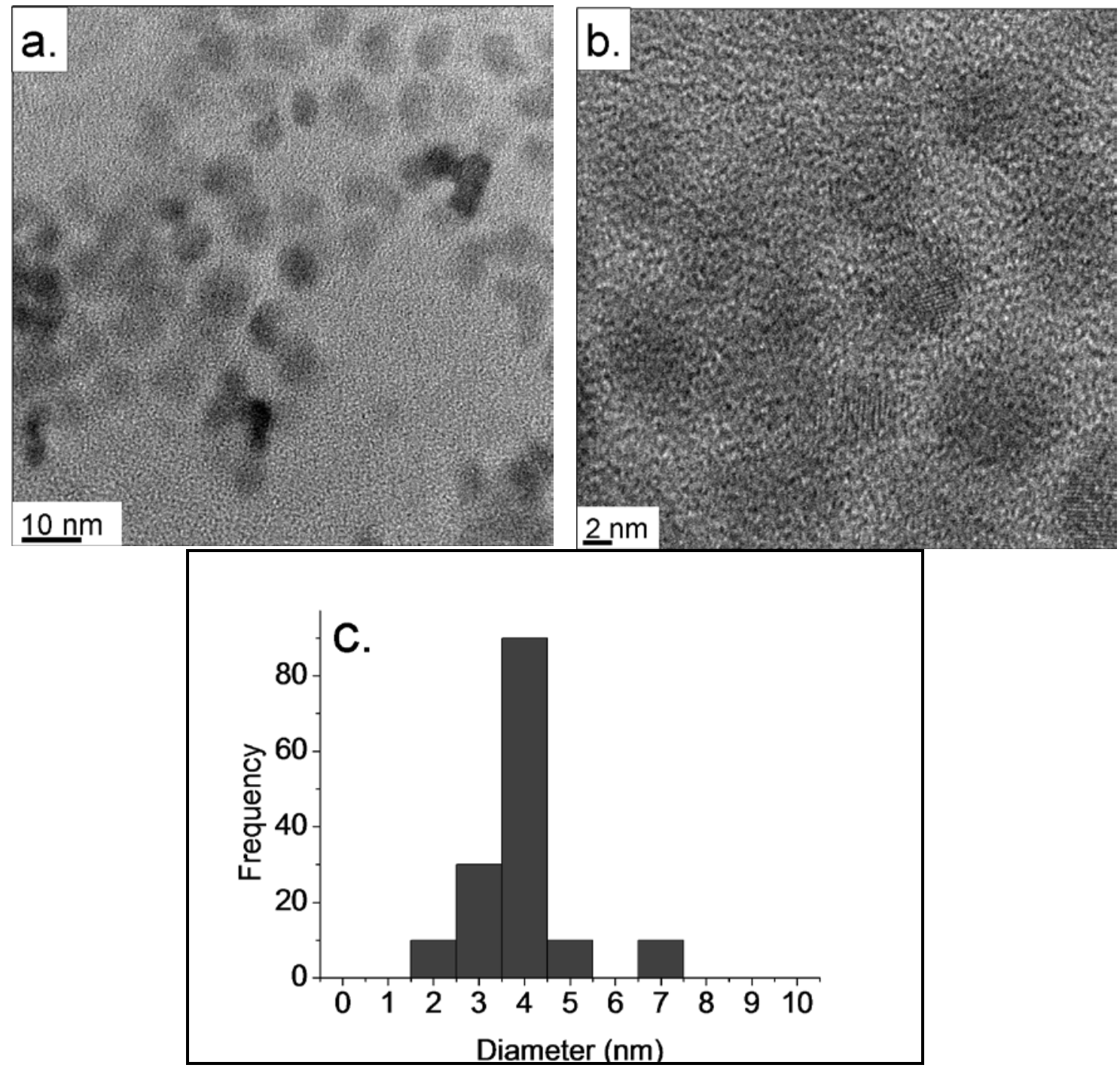

Figure 3-2: (a); Low resolution and (b); high resolution TEM micrograph of silicon quantum dot capped with 1-hexene respectively. (c): size distribution graph of the silicon quantum dots capped with 1-hexene $(n=200)$

From EDS analysis, existence of silicon was confirmed. Atomic \% of silicon was 20.8\%. Neither of platinum nor bromine peak were observed which indicates the nanoparticles observed in TEM micrograph were crystalline silicon. This spectrum suggests that the nanoparticles seen in Figure 3-3 were composed of mainly silicon. 


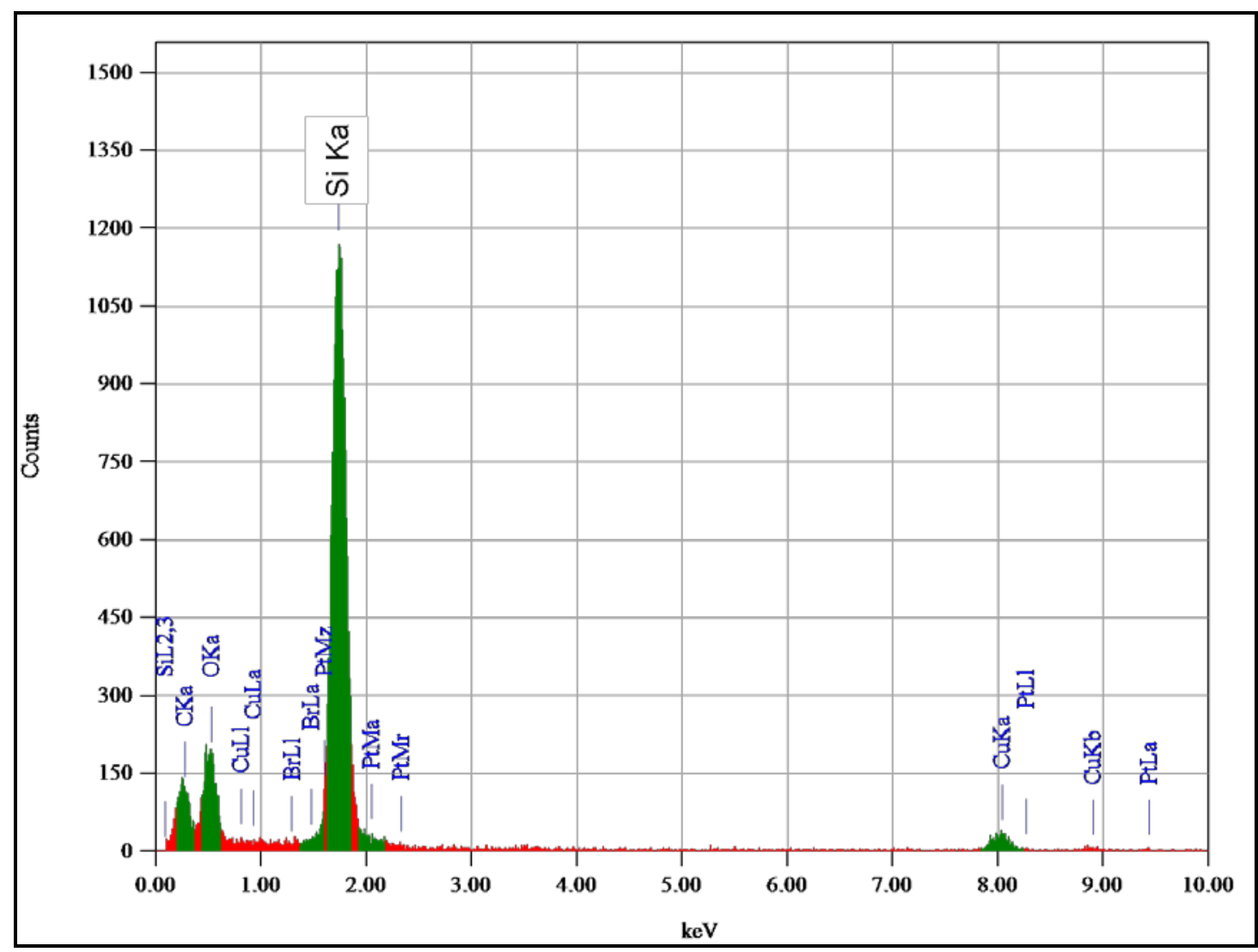

Figure 3-3: An EDS spectrum obtained from the sample of experiment 3.

The surface molecules of the nanoparticles were characterised by FTIR (Figure 3-4) and NMR (Figure 3-5) spectroscopy. In the FTIR spectrum, the peaks observed at $1460 \mathrm{~cm}^{-1}$ and $1260 \mathrm{~cm}^{-1}$ are attributed to the vibrational scissoring and symmetric bending of $\mathrm{Si}-\mathrm{CH}_{2}$ respectively. These peaks indicate that the double bond of 1hexene was attached to silicon. The peaks around $2850 \mathrm{~cm}^{-1}$ to $2925 \mathrm{~cm}^{-1}$ represent C$\mathrm{CH}_{2}$ symmetrical and asymmetrical stretching. The broad peak around $3400 \mathrm{~cm}^{-1}$ is probably due to water. ${ }^{7}$ 


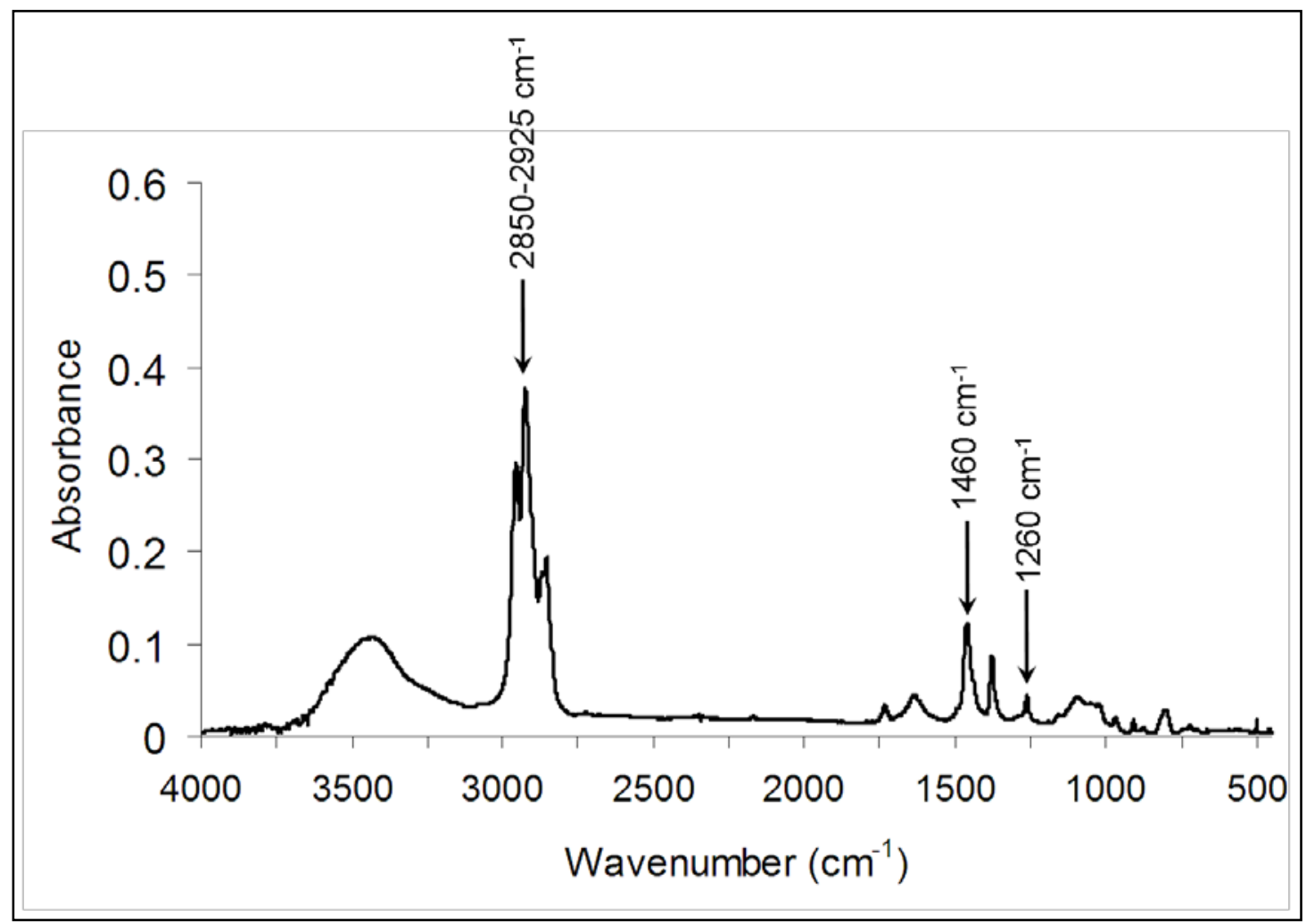

Figure 3-4: FTIR spectrum of the sample obtained from experiment 3 . The peaks suggest that the double bond of 1-hexene is attached to silicon.

In the NMR spectrum of free 1-hexene, signals are observed at $5.80 \mathrm{ppm}, 4.96 \mathrm{ppm}$, $4.92 \mathrm{ppm}$ representing the terminal double bond, however these signals cannot be seen in the NMR spectrum of the sample from experiment 3. Also the signal at 2.06 ppm from the NMR spectrum of free 1-hexene has disappeared in the NMR of the sample. The triplet at $0.814 \mathrm{ppm}$ integrates for 5 protons, indicating the terminal methyl group and $\mathrm{CH}_{2}$ next to silicon. Usually the methylene signal appears further down field, around $1.0 \mathrm{ppm}$, however by being attached to silicon quantum dot these protons are probably more shielded. The signal observed at $1.192 \mathrm{ppm}$ integrates for the 8 protons attached to the carbons between the methyl group and $\mathrm{Si}-\mathrm{CH}_{2}$. 

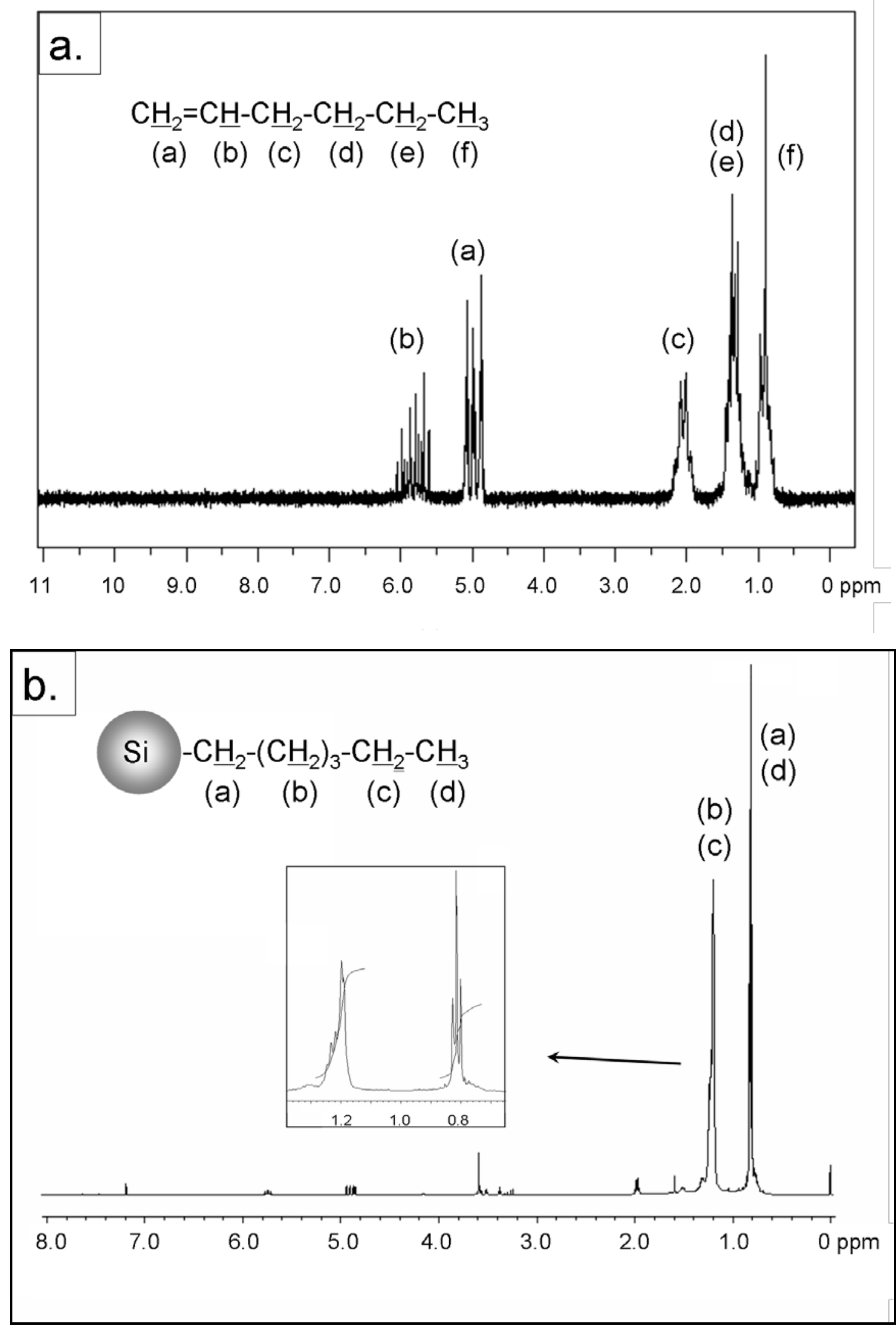

Figure 3-5: ${ }^{1} \mathrm{H}$ NMR spectra of (a); free 1-hexene and (b); the sample obtained from experiment 1 . Inset figure shows the details of the signals at $0.81 \mathrm{ppm}$ and $1.19 \mathrm{ppm}$. 
1-hexene

${ }^{1} \mathrm{H}$ NMR (500MHz, $\left.\mathrm{CDCl}_{3}\right)$

$\delta: 0.90 \mathrm{ppm}(3 \mathrm{H}, \mathrm{t}, \mathrm{H}-(\mathrm{f}), J=6.5 \mathrm{~Hz}), \delta: 1.34 \mathrm{ppm}(4 \mathrm{H}, \mathrm{m}, \mathrm{H}-(\mathrm{d}),(\mathrm{e})), \delta: 2.06 \mathrm{ppm}(2 \mathrm{H}$, q, H-(c) $J=6.7 \mathrm{~Hz}), \delta: 4.94$ ppm (2H, dd, H-(a) $J=1.75,6.9 \mathrm{~Hz}), \delta: 5.80$ ppm (1H, m, $\mathrm{H}-(\mathrm{b}))$

Silicon quantum dots capped with 1-hexene

${ }^{1} \mathrm{H}$ NMR (500MHz, $\left.\mathrm{CDCl}_{3}\right)$

$\delta: 0.81 \mathrm{ppm}(5 \mathrm{H}, \mathrm{t}, \mathrm{H}-(\mathrm{a}),(\mathrm{d})), \delta: 1.19 \mathrm{ppm}(8 \mathrm{H}, \mathrm{m}, \mathrm{H}-(\mathrm{b}),(\mathrm{c}))$

From the experiments 2 and 3, the effect of different surfactants on particle size and shape was investigated. Both samples from experiment 2 and 3 showed spherical nanoparticles. Nanoparticles size distribution was more uniform by when TOAB was used as a surfactant. The discussion of the result will be mentioned later in discussion section.3-5.

\section{3-1-3. Silicon quantum dots capped with 1,9-decadiene: Experiment 4}

Silicon quantum dots capped with 1,9-decadiene were synthesised to ascertain if one of the terminal double bonds can remain on the surface of the silicon quantum dot after capping process. Diene molecules can expand the possibilities of surface modification for the silicon quantum dot by reacting the terminal double bonds that remains on the terminal end of the capping agents with other functional groups. 

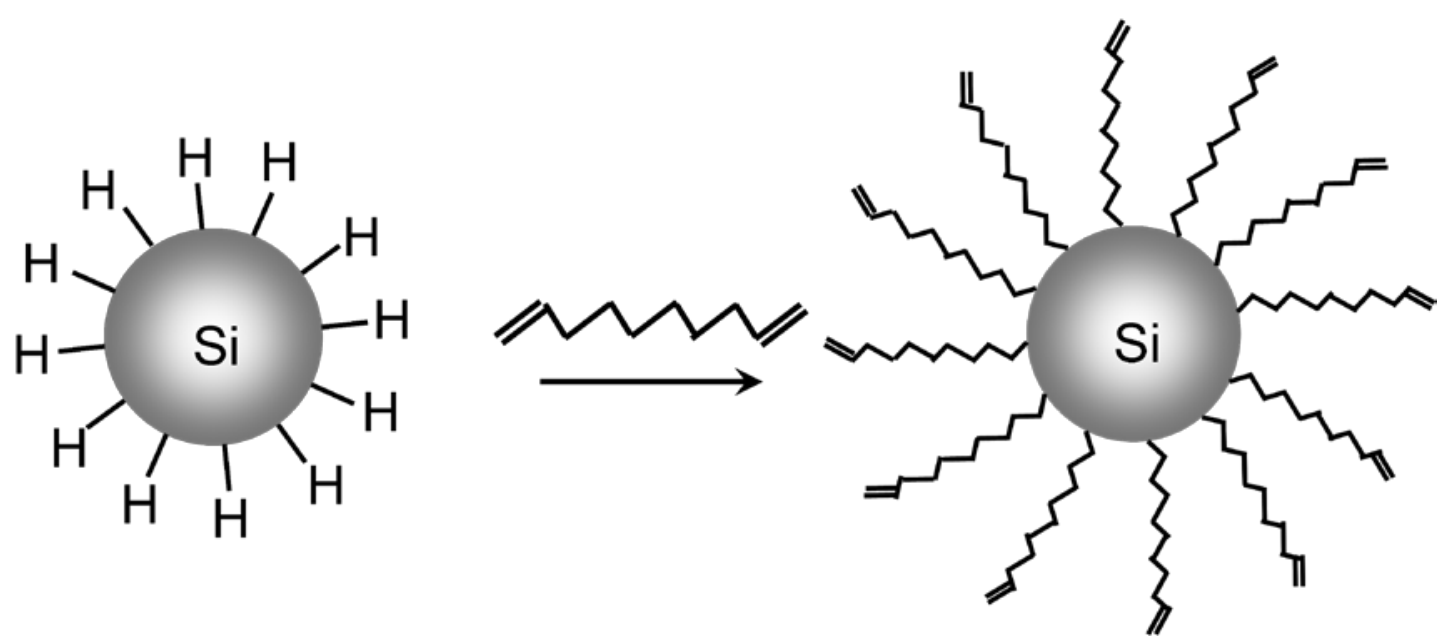

Scheme 3-1-3: Reaction scheme for 1,9-decadiene capping of the silicon quantum dot

\section{Experimental}

$0.77 \mathrm{~g}$ of pentaethylene glycol monododecyl ether $\left(\mathrm{C}_{12} \mathrm{E}_{5}\right) 0.3 \mathrm{~mL}(0.002 \mathrm{~mol})$ of silicon tetrabromide $\left(\mathrm{SiBr}_{4}\right), 3 \mathrm{~mL}$ of $1.0 \mathrm{M}$ lithium triethylborohydride (superhydride)were used in this experiment. After the reduction, anhydrous methanol (2 mL) was added to quench any remaining superhydride. Surface passivation was conducted with $5 \mathrm{~mL}$ of anhydrous 1,9-decadiene using hexachloro platinic acid. The solution was transferred into a separation funnel and $\mathrm{N}$-methylformamide was added to remove impurities. The silicon quantum dots were extracted by liquid-phase separation. The hexane layer was collected and dried under vacuum then the yellow oil was re-dispersed in anhydrous hexane.

\section{Results}

As may be seen in Figure 3-6, lattice fringes were observed by TEM. However the aggregates are not spherically shaped. No SAED was obtained from this area. Since the product cannot be obtained as solid XRD analysis cannot be conducted. The shapes of the aggregates were not spherical therefore average size and size distribution could not be measured.

The EDS spectrum in Figure 3-7 shows the existence of silicon and indicates that these nanoparticles are composed mainly of silicon. The atomic \% of silicon was 18.9\%. It is difficult to determine the amount of carbon from capping molecules by 
quantitative data of EDS analysis since the TEM grid is coated by carbon. Platinum peak from catalyst was hardly seen in this spectrum.

In the NMR spectrum the quartet at $4.89 \mathrm{ppm}$ and multiplet at $5.74 \mathrm{ppm}$ indicate the terminal double bond of the moieties on the surface of the silicon quantum dots (H-(e) and $\mathrm{H}-(\mathrm{d})$ respectively). It also has the triplet at $0.81 \mathrm{ppm}, \mathrm{H}-(\mathrm{a})$, which does not show in the spectrum of free 1,9-decadiene molecule. This is possibly from the methylene protons next to silicon. The multiplet at $1.21 \mathrm{ppm}$ indicates the protons of carbon chain at the position (b). H-(c) can be observed at $1.97 \mathrm{ppm}$ as quartet. However the integrals for $\mathrm{H}-(\mathrm{a})$ and $\mathrm{H}-(\mathrm{b})$ did not match to the theoretical ratio of the protons. This could be due to the overlap of the signals of surface molecule and impurities such as $\mathrm{C}_{12} \mathrm{E}_{5}$ or the aggregation of the nanoparticles by being bridged with the terminal double bond. To prevent this reaction, shorter carbon chain diene molecule, 1,5hexadiene, was used in next experiment.

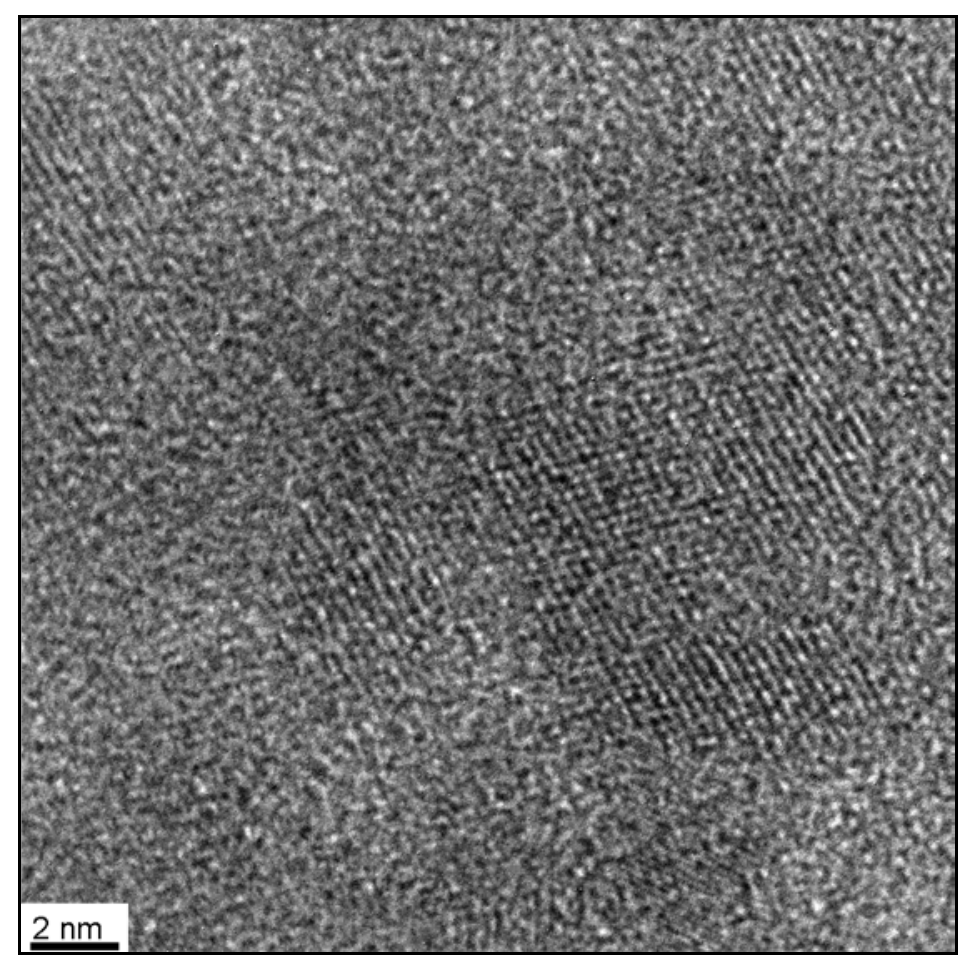

Figure 3-6: High resolution TEM micrograph of the sample obtained from experiment 4. 


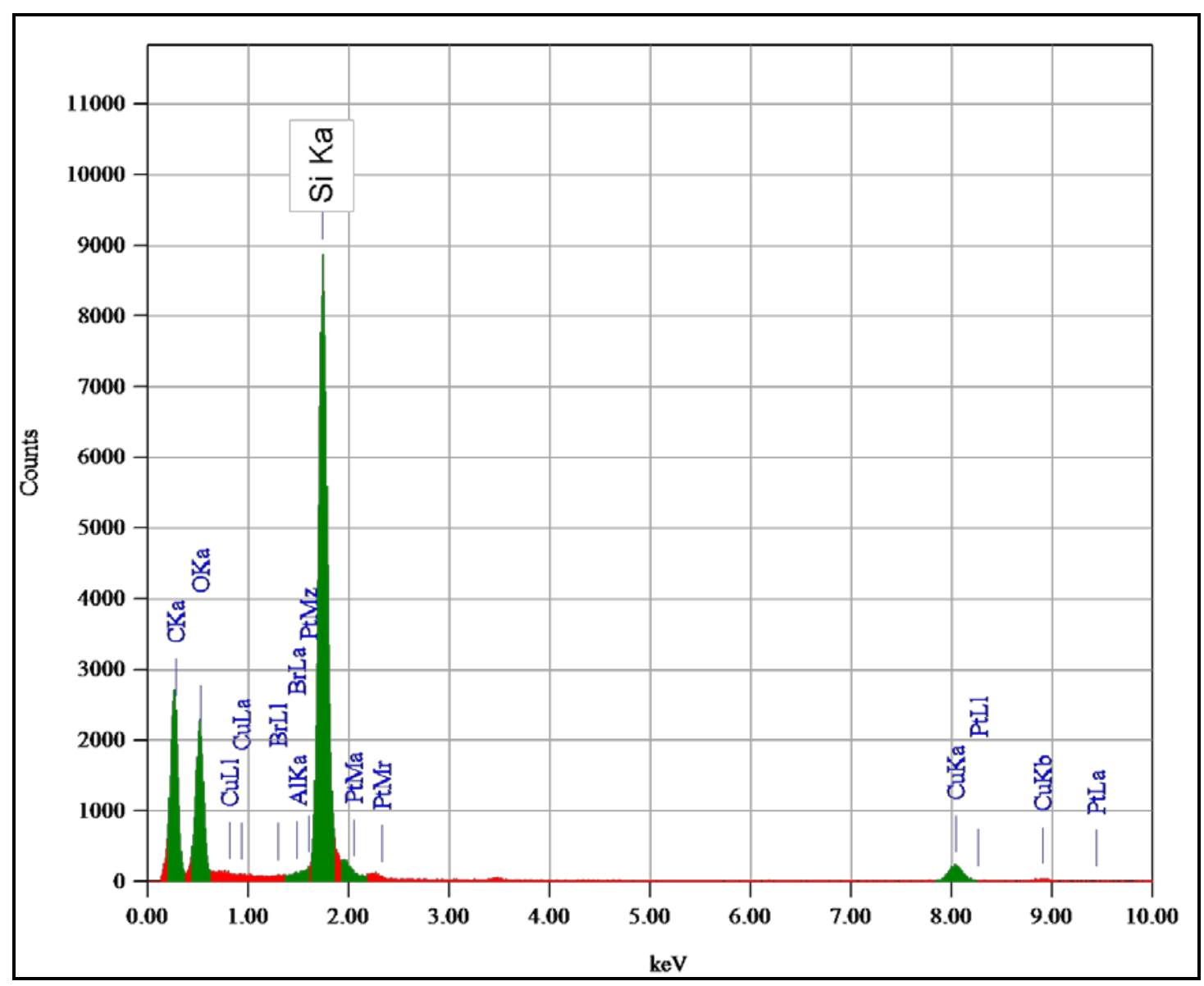

Figure 3-7: An EDS spectrum obtained from the sample of experiment 4.

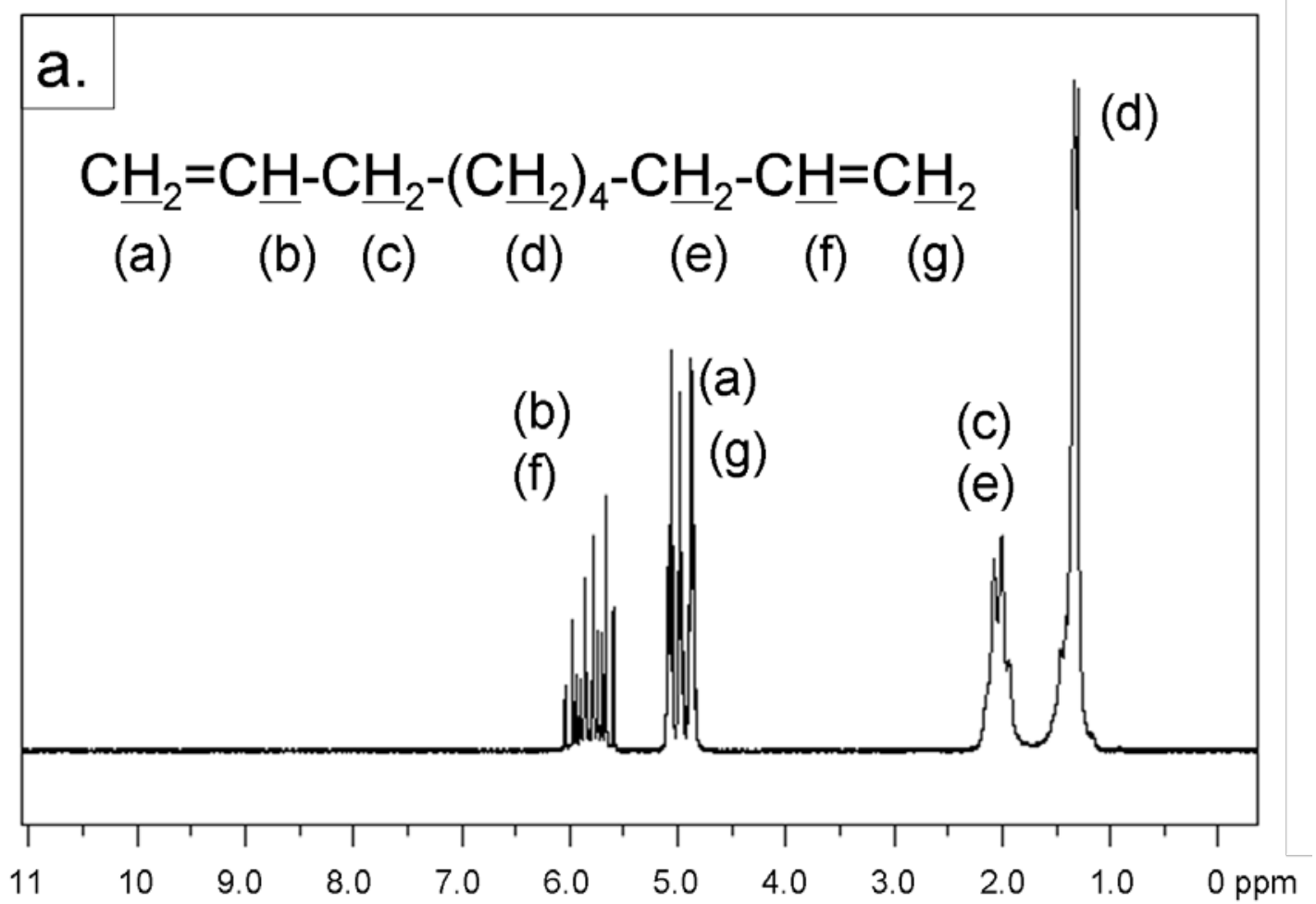




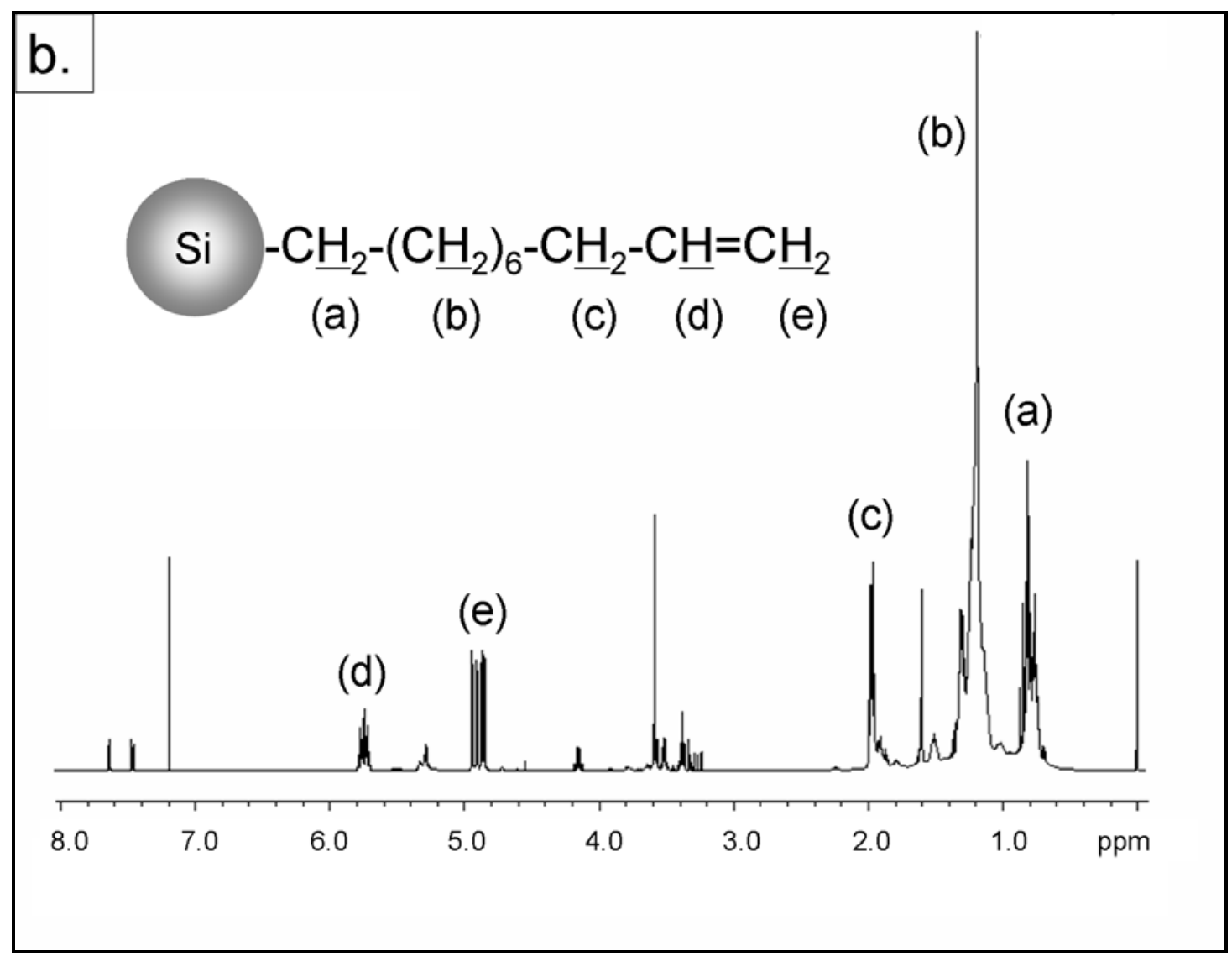

Figure 3-8: ${ }^{1} \mathrm{H}$ NMR spectrum of (a); free 1,9-decadiene and (b); the sample obtained from experiment 4 . The inset figure is a representation of the silicon quantum dot capped with 1,9-decadiene.

1,9-decadiene

${ }^{1} \mathrm{H}$ NMR (500MHz, $\mathrm{CDCl}_{3}$ )

$\delta: 1.62-1.09$ ppm (8H, m, H-(d)), $\delta: 2.03$ ppm (4H, m, H-(c), (e)), $\delta: 4.92$ ppm (2H, d, H-(a),(g) J=0.57 Hz), $\delta: 4.98$ ppm (2H, d, H-(a),(g) J=1.18 Hz), $\delta: 5.79$ ppm (2H, m, H-(b),(f))

Silicon quantum dots capped with 1,9-decadiene

${ }^{1} \mathrm{H}$ NMR (500MHz, $\left.\mathrm{CDCl}_{3}\right)$ $\delta: 4.91-4.94$ ppm (2H, dd, H-(e), J=1.2, $17.1 \mathrm{~Hz}) \delta: 5.75$ ppm (1H, m, H-(d))

The TEM and EDS results shown above imply crystalline silicon aggregates were synthesised, however the TEM micrograph did not show spherical nanoparticles. The possible reasons could be due to the bridging of the capping agent since it has two terminal double bonds and 8 carbons in between. 


\section{3-1-4. Silicon quantum dot capped with 1,5-hexadiene}

After the conformation of the presence of the double bonds on the terminal end of the capping agent of the silicon quantum dots from experiment 4, 1,5-hexadiene was chosen as surface capping molecule. Since 1,5-hexadiene is shorter than 1,9decadiene, the risk of bridging two nanoparticles with the terminal double bond can be reduced because of the steric hindrance of quantum dots. Also it can produce smaller nanoparticles diameter than 1,9-decadiene because of the shorter carbon chain. The terminal double bonds remaining on the capping agents on the silicon quantum dot were further modified to other functional groups. This will be described in chapter 4.

In this experiment, two different ways for capping the silicon quantum dots were tested to compare difference in the results such as particle size, size distribution and impurities. Pt catalyst capping has been commonly applied as the capping method in a microemulsion system. ${ }^{8,9,10}$ In contrast, UV-irradiation capping was applied as a new capping method. ${ }^{25-30}$ This method can reduce the risk of producing platinum nanoparticles if excess of the reducing agent was left in the reaction mixture.
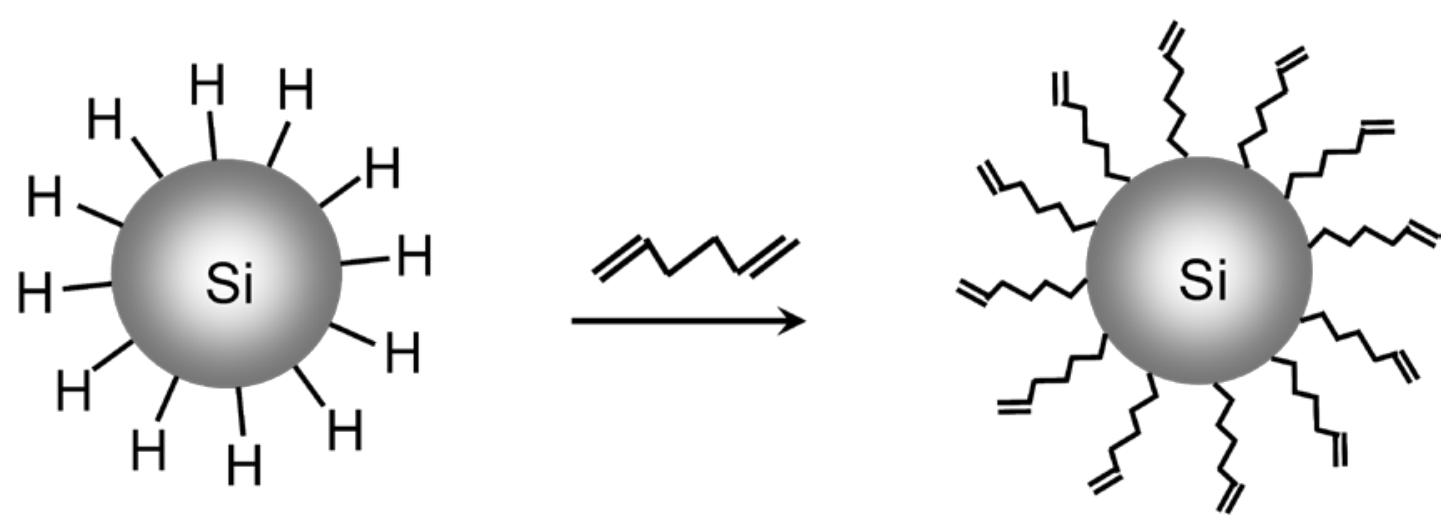

Scheme 3-1-4: Reaction scheme for 1,5-hexadiene capping of the silicon quantum dot

\section{Experiment 5: Pt catalyst capping}

\section{Experimental}

$1.0 \mathrm{~g}$ of tetraoctylammonium bromide (TOAB), $0.3 \mathrm{~mL}(0.0024 \mathrm{~mol})$ of silicon tetrabromide $\left(\mathrm{SiBr}_{4}\right)$ and $5 \mathrm{~mL}$ of $1.0 \mathrm{M}$ lithium triethylborohydride (superhydride) 
were used in this experiment. Also $50 \mathrm{~mL}$ of anhydrous toluene was used as a solvent. After the reduction methanol (4 mL) was added to quench any remaining superhydride. Surface passivation was conducted with $3 \mathrm{~mL}$ of anhydrous 1,5hexadiene using hexachloro platinic acid. The reaction mixture was transferred into a separation funnel and silicon quantum dots were extracted by liquid-phase separation. The hexane layer was collected and dried up under vacuum and re-dissolved in anhydrous hexane.

\section{Results}

The sample obtained from Pt catalyst capping system was characterised by TEM and EDS analysis. As may be seen in Figure 3-9(a), spherical aggregates were observed by TEM in an average size of $4.8 \pm 2.7 \mathrm{~nm}$. The smaller nanoparticles have diameter around 3-5 nm which is close to the Bohr radius of silicon. Besides the small spherical nanoparticles, larger spherical shades were observed. In the EDS analysis $3.5 \%$ of silicon was detected and neither platinum nor bromine peaks were observed. It was too difficult to determine the silicon peak observed by EDS analysis was from the sample or the EDS detector. No SAED was obtained from this sample. Since the amount of platinum catalyst is very small the spherical nanoparticles observed from TEM image are more likey to be silicon quantum dots. However since no SAED was obtained to determine silicon quantum dots there is still the possibility of forming platinum nanoparticles. 

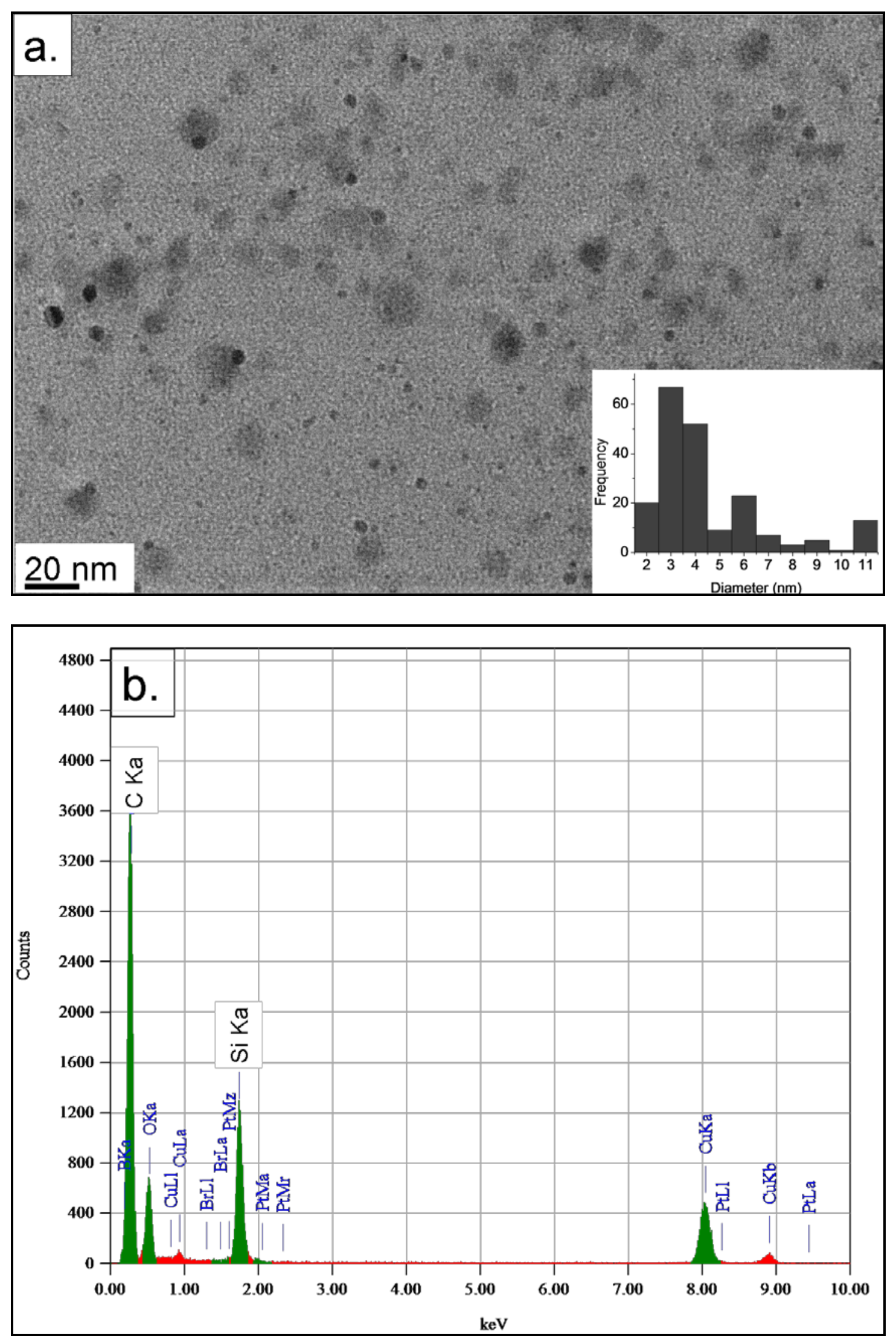

Figure 3-9: (a); a TEM micrograph of silicon quantum dots capped with 1,5hexadiene, (b); EDS spectrum of the area of TEM image (a) 


\section{Experiment 6: UV-irradiation capping}

\section{Experimental}

$0.05 \mathrm{~g}$ of pentaethylene glycol monododecyl ether $\left(\mathrm{C}_{12} \mathrm{E}_{5}\right)$ was dissolved in $50 \mathrm{~mL}$ of anhydrous toluene and $0.3 \mathrm{~mL}(0.0024 \mathrm{~mol})$ of $\mathrm{SiBr}_{4}, 5 \mathrm{~mL}$ of $2.0 \mathrm{M}$ lithium borohydride were used in this experiment. Surface passivation was conducted by UVirradiation method for 5 hours. . N-methylformamide was added to the reaction mixture in a separation funnel and silicon quantum dots were extracted by liquidphase separation. The hexane layer was collected and dried under vacuum then the yellow oil was re-dispersed in anhydrous hexane.

\section{Results}

The sample was characterised by TEM, SAED and EDS. The surface molecules were analysed by FTIR and ${ }^{1} \mathrm{H}$ NMR. A low resolution TEM micrograph, a high resolution TEM micrograph and SAED are shown in Figure 3-10 (a), (b), and (c) respectively.

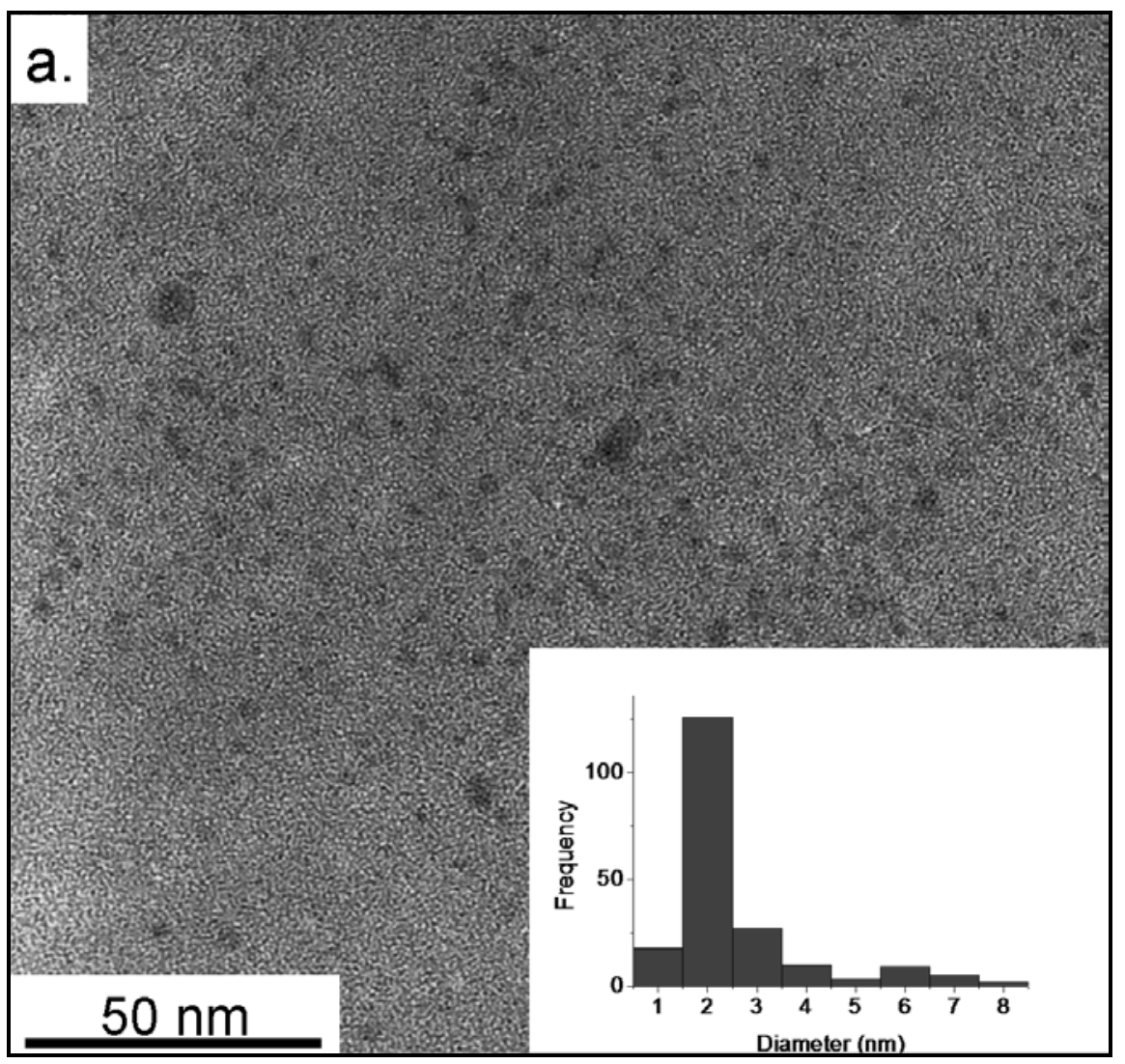




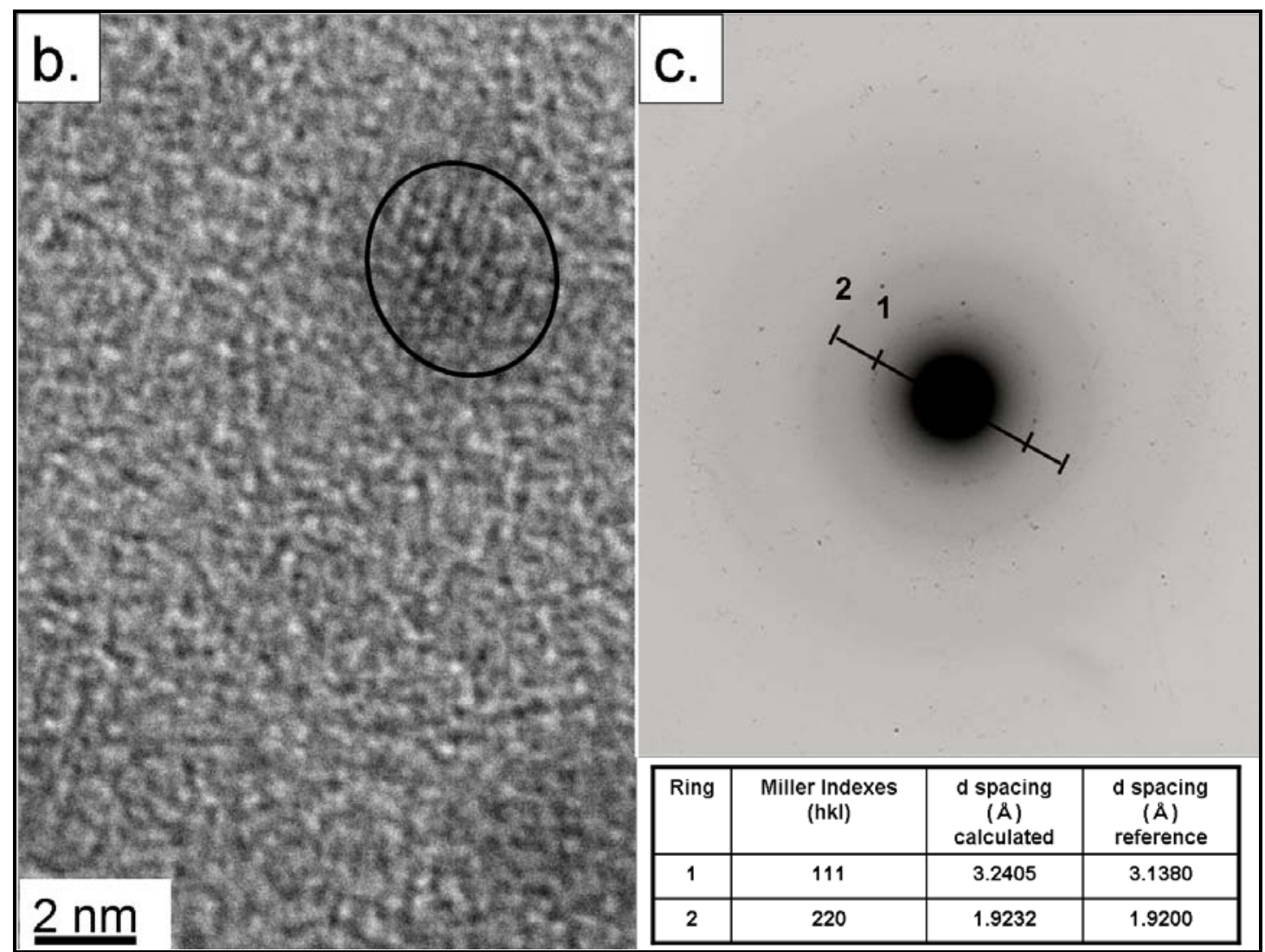

Figure 3-10: (a): low resolution TEM micrograph of silicon quantum dots obtained from experiment 6 (b): high resolution TEM micrograph of silicon quantum dot obtained from experiment 6 (c) Indexed SAED patterns of silicon quantum dots obtained from experiment 6

Relatively mono dispersed spherical nanoparticles were observed by TEM in an average size of $2.5 \pm 1.6 \mathrm{~nm}$ (Figure 3-10 (a)) . From the high resolution TEM image, lattice fringes were observed. The first and second rings in the SAED taken at the same region as the TEM micrograph (a) show (111) and (220) phases of crystalline silicon respectively. This implies successful synthesis of crystalline silicon nanoparticles. From EDS analysis, the amount of silicon was $7.6 \%$ which is much larger compared to silicon from EDS detector.

The sample was further characterised by FTIR and NMR. The results of FTIR and NMR analysis were shown in Figure 3-12 and Figure 3-13 respectively.

The FTIR spectrum shows the terminal double bond of the surface moiety of the silicon quantum dot at $1640 \mathrm{~cm}^{-1}$ which corresponds to $\mathrm{C}=\mathrm{C}$ stretching and also $\mathrm{C}=\underline{\mathrm{C}}$ $\underline{\mathrm{H}}$ stretching which can be seen as the small peak at $3077 \mathrm{~cm}^{-1} .^{31}$ Also vibrational scissoring and symmetrical bending of Si-CH $\mathrm{CH}_{2}$ were shown by the peaks at $1460 \mathrm{~cm}^{-1}$ and $1260 \mathrm{~cm}^{-1} .{ }^{8}$ These peaks indicate that 1,5-hexadiene was attached to a silicon 
quantum dot and one of the terminal double bonds remained on the surface. The peaks from $2855 \mathrm{~cm}^{-1}$ to $2957 \mathrm{~cm}^{-1}$ represent C-CH $\mathrm{CH}_{2}$ symmetrical, asymmetrical stretching. ${ }^{8,31}$

The terminal double bond on the silicon quantum dot surface was also confirmed by ${ }^{1} \mathrm{H}$ NMR spectroscopy. The quartet at $4.90 \mathrm{ppm}$ and multiplet at $5.72 \mathrm{ppm}$ clearly indicate the existence of the double bond on the terminal end of the silicon quantum dots (H-(e) and H-(d) respectively). The triplet at $0.81 \mathrm{ppm}$ and multiplet at $1.22 \mathrm{ppm}$ did not show in the spectrum of free 1,5-hexadiene (data not shown). These signals possibly represent $\mathrm{H}$-(a) and $\mathrm{H}$-(b) from the surface bonding on the silicon quantum dot. The chemical shift of H-(a) and H-(b) is due to the breaking of the double bond and attachment of the chain to silicon. H-(c) signal can be seen at $1.96 \mathrm{ppm}$. However the integrals of the signals of H-(a), H-(b), and H-(c) did not match to the number of protons of $\mathrm{H}-(\mathrm{a}), \mathrm{H}-(\mathrm{b})$ and $\mathrm{H}-(\mathrm{c})$. This could be due to overlapping with the signals of impurities such as the surfactant, TOAB. The structure of TOAB and ${ }^{1} \mathrm{H}$ NMR spectrum can be seen in Figure 3-14. As may be seen in Figure 3-14, TOAB has protons signals in the same region as $\mathrm{H}-(\mathrm{a}), \mathrm{H}-(\mathrm{b})$ and $\mathrm{H}-(\mathrm{c})$. 


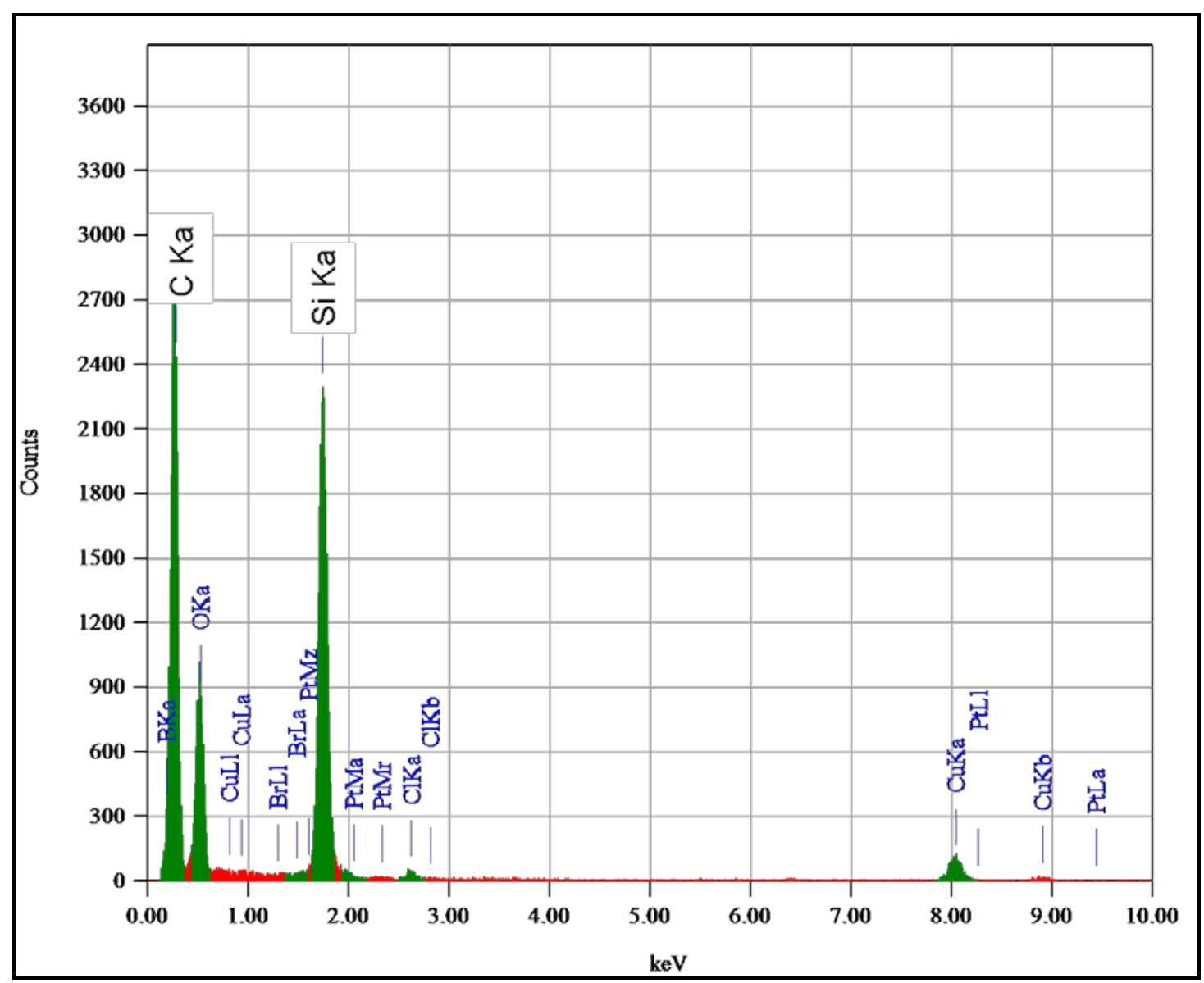

Figure 3-11: EDS spectrum of the sample obtained from experiment 6

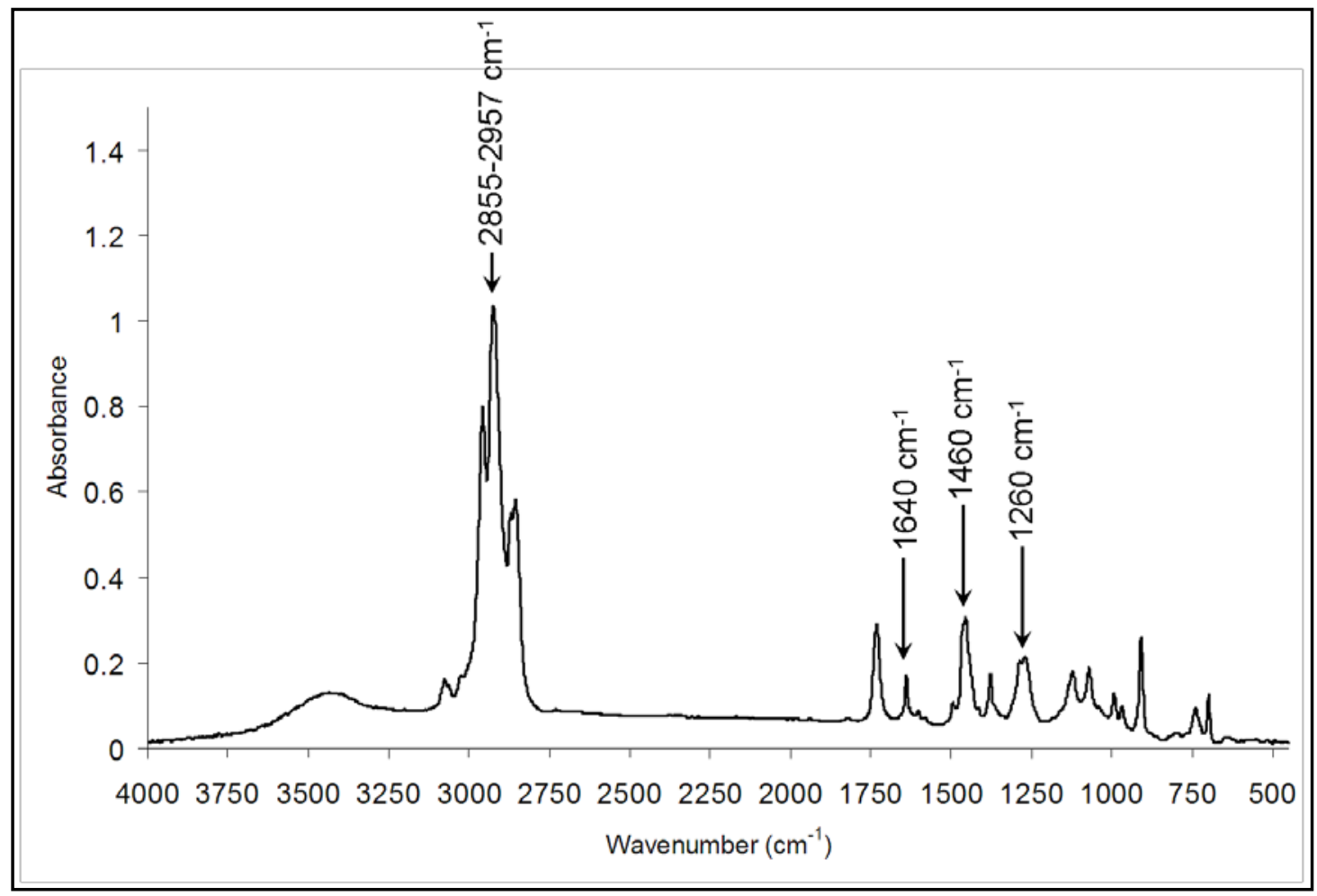

Figure 3-12: FTIR spectrum of the sample obtained from experiment 6 

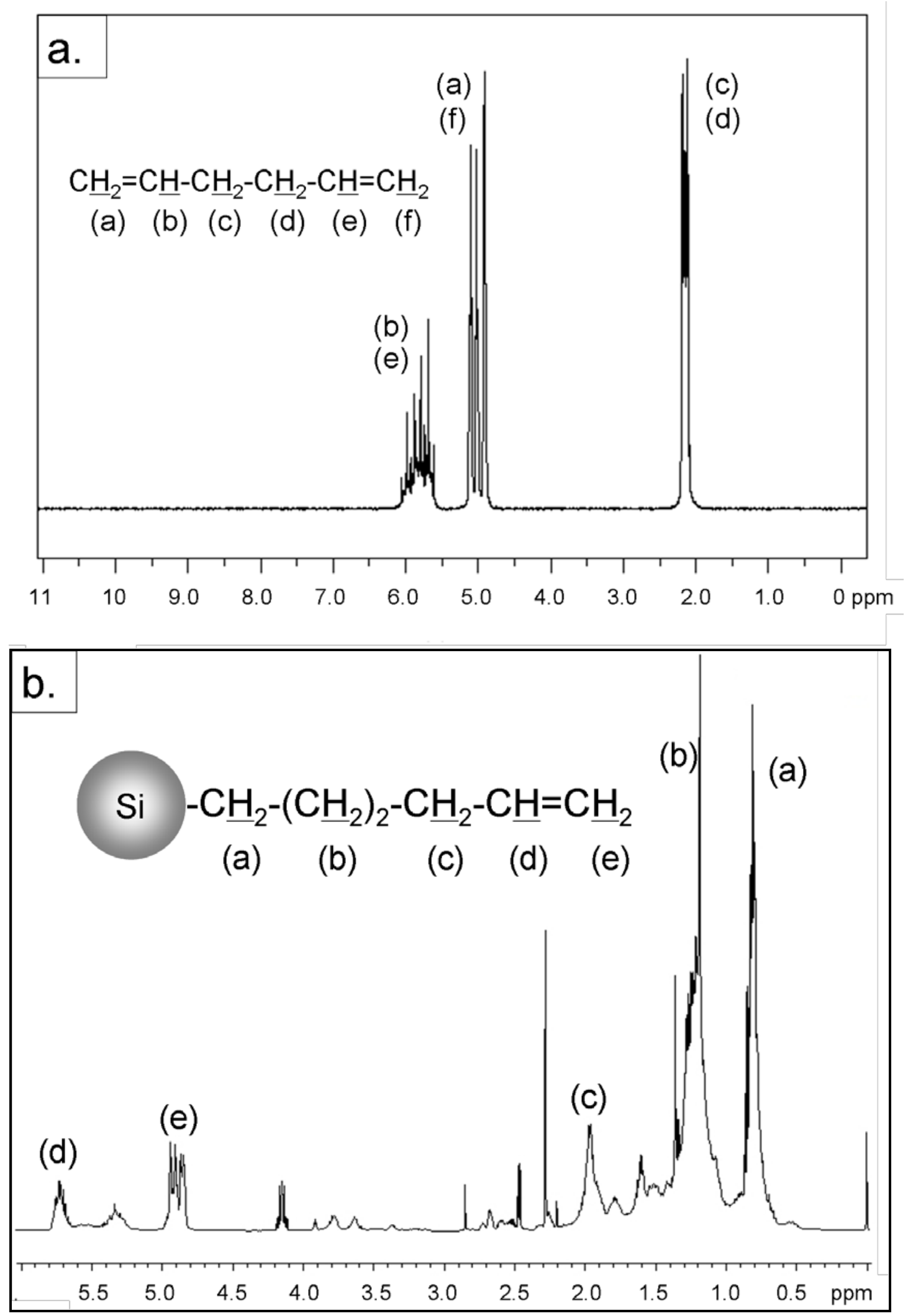

Figure 3-13: ${ }^{1} \mathrm{H}$ NMR spectrum of (a); free 1,5-hexadiene and (b); the silicon quantum dots capped with 1,5-hexadiene obtained from experiment 6 . 
1,5-hexadiene

${ }^{1} \mathrm{H}$ NMR (500MHz, $\left.\mathrm{CDCl}_{3}\right)$

$\delta: 2.15$ ppm (4H, t, H-(c),(d), $J=1.3 \mathrm{~Hz}), \delta: 4.95$ ppm (2H, d, H-(a), (f), $J=0.9 \mathrm{~Hz}$ ),

$\delta: 4.99$ ppm (2H, d, H-(a),(f) $J=2.3 \mathrm{~Hz}), \delta: 5.81$ ppm (2H, m, H-(b),(e))

Silicon quantum dots capped with 1,5-hexadiene

${ }^{1} \mathrm{H}$ NMR (500MHz, $\mathrm{CDCl}_{3}$ )

$\delta: 1.96 \mathrm{ppm}(2 \mathrm{H}, \mathrm{m}, \mathrm{H}-(\mathrm{c})), \delta: 4.90 \mathrm{pm}(2 \mathrm{H}, \mathrm{dd}, \mathrm{H}-(\mathrm{e}), J=9.0,36.4 \mathrm{~Hz}), \quad \delta$ :

5.72ppm (1H, m, H-(d))

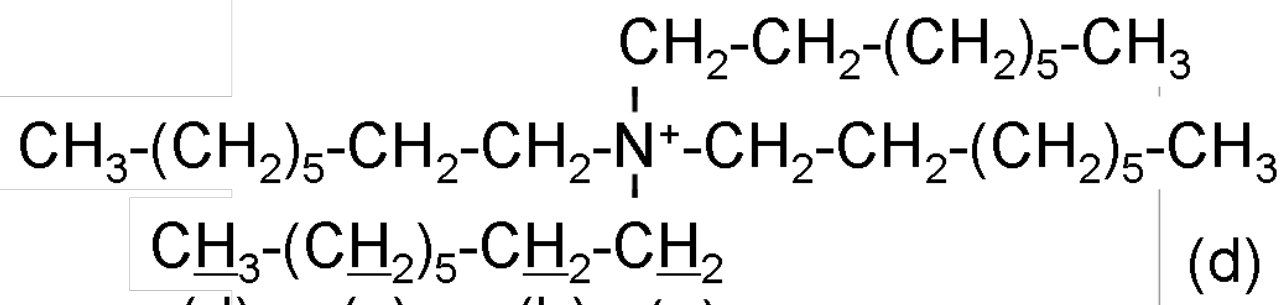

(d) (c) (b) (a)

(c)

(a)

(b)

4.0

3.0

2.0

1.0

ppm

Figure 3-14: ${ }^{1} \mathrm{H}$ NMR spectrum of TOAB 
TOAB

${ }^{1} \mathrm{H}$ NMR (500MHz, $\left.\mathrm{CDCl}_{3}\right)$

$\delta: 0.87 \mathrm{ppm}(12 \mathrm{H}, \mathrm{t}, \mathrm{H}-(\mathrm{d}), J=7.1 \mathrm{~Hz}), \delta: 1.27 \mathrm{pm}(40 \mathrm{H}, \mathrm{m}, \mathrm{H}-(\mathrm{c})),, \delta: 1.65 \mathrm{ppm}$

(8H, m, H-(b)), 3.38 ppm (8H, t, H-(a), J=8.6 Hz)

Both platinum catalyst capping and UV-irradiation capping methods produced spherical nanoparticles. In the sample obtained from $\mathrm{Pt}$ catalyst capping, the nanoparticles could not be confirmed as crystalline silicon since SAED could not be obtained and EDS analysis did not show reasonable amount of silicon. Also the nanoparticles were poly disperse. By comparison, the sample obtained from UVirradiation capping showed relatively monodisperse nanoparticles. From SAED, it was confirmed the nanoparticles were crystalline silicon. Also the results from the FTIR and ${ }^{1} \mathrm{H}$ NMR analysis showed the evidence of attachment of 1,5-hexadiene onto the surface of the silicon quantum dots.

The spherical aggregates observed from platinum catalyst capping still showed the presence of impurities even after purification. This could be due to side products from platinum catalyst. Also if any reducing agent was left in the reaction mixture after the reduction step, the platinum catalyst will be reduced to form platinum nanoparticles. Platinum catalyst capping method leaves possibilities of forming platinum nanoparticles, therefore it is difficult to confirm that the aggregates are crystalline silicon without SAED. Compared to platinum catalyst capping, UV-irradiation method has less possibility to produce impurities since $254 \mathrm{~nm}$ specifically promote hydrosilylation. ${ }^{28}$

\section{3-1-5. Silicon quantum dots capped with allylamine}

Silicon quantum dots capped with allylamine were synthesised to produce silicon quantum dots with amino groups on the surface. The intention of this was to broaden the possibilities of attaching bio-functional molecules on the surface. Since amino groups have a lone pair of electrons on the nitrogen atom, they can react as a nucleophile, forming an amide bond with an active ester. In the following section, biotinylation of the silicon quantum dots using sulfo-NHS-LC-biotin will be described. 
Since the bond strength of Si-N bond (355 kJ/mol) is slightly weaker than Si-C bond (360 kJ/mol) therefore the terminal amine group was expected to remain at the terminal end of the silicon quantum dot. Allylamine was chosen as the capping agent because of its low price and easy availability. The Pt catalyst capping and the UVirradiation capping methods were conducted to compare the results.
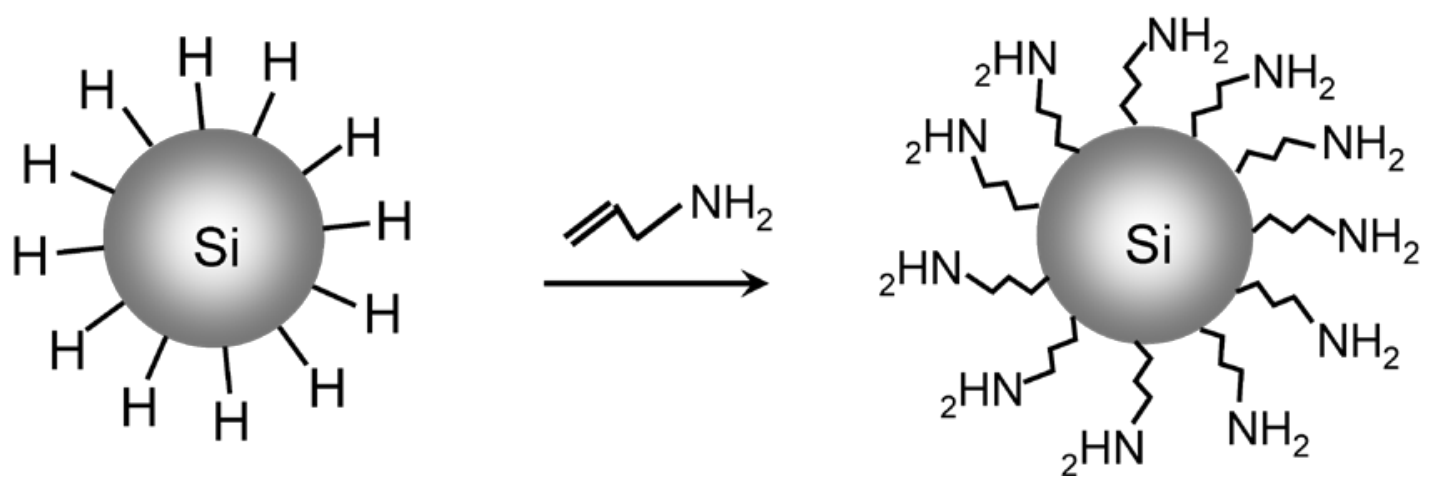

Scheme 3-1-5: Reaction scheme of allylamine capping for silicon quantum dots

\section{Experiment 7: Pt catalyst capping}

\section{Experimental}

$0.25 \mathrm{~g}$ of pentaethylene glycol monododecyl ether $\left(\mathrm{C}_{12} \mathrm{E}_{5}\right) 0.3 \mathrm{~mL}(0.0026 \mathrm{~mol})$ of silicon tetrachloride $\left(\mathrm{SiCl}_{4}\right)$ and $4 \mathrm{~mL}$ of $2.0 \mathrm{M}$ lithium borohydride were used in this experiment. Also $50 \mathrm{~mL}$ of anhydrous hexane was used as a solvent.. Surface passivation was conducted with $5 \mathrm{~mL}$ of allylamine using platinum norbornene catalyst Platinum norbornene was applied in this experiment because it has better solubility in the organic solvent compared to hexachloro platnic acid. After the reaction the solution was transferred into a separation funnel and $20 \mathrm{~mL}$ of methanol was added. After three times of liquid-phase separation, silicon quantum dots were extracted into methanol layer.

\section{Results}

No spherical nanoparticles were observed from TEM micrograph. Cubic shape crystals and some oval shape aggregates were observed. EDS analysis showed the existence of chlorine. The result indicates that the cubic crystals are most likely lithium chloride. 
Since no silicon peak was detected from EDS analysis the oval shape aggregates are possibly platinum nanoparticles produced from platinum catalyst. This is possibly due to the reduction of the platinum catalyst. When the reducing agent was added, partial

of the precursor formed silane gas which went off. ${ }^{32,33}$ If excess reducing agent left in the reaction mixture may reduce the Pt catalyst to form platinum nanoparticles. Therefore the preparation was unsuccessful.

\section{Experiment 8: UV-irradiation capping}

\section{Experimental}

$0.25 \mathrm{~g}$ of pentaetylene glycol monododecyl ether $\left(\mathrm{C}_{12} \mathrm{E}_{5}\right) 0.3 \mathrm{~mL}(0.0026 \mathrm{~mol})$ of silicon tetrachloride $\left(\mathrm{SiCl}_{4}\right), 4 \mathrm{~mL}$ of $2.0 \mathrm{M}$ lithium borohydride were used in this experiment. . Surface passivation was conducted by UV-irradiation method with $5 \mathrm{~mL}$ of allylamine for 4 hours. The white precipitate was removed by filtration and the solution was dried under vacuum then the yellow oil was re-dispersed in $10 \mathrm{~mL}$ of methanol. The precipitate was removed by filtration with a millipore filter $(0.45 \mu \mathrm{m}$ Durapore Membrane filter) and the silicon quantum dots were purified by size exclusion column chromatography (LH20). 


\section{Results}

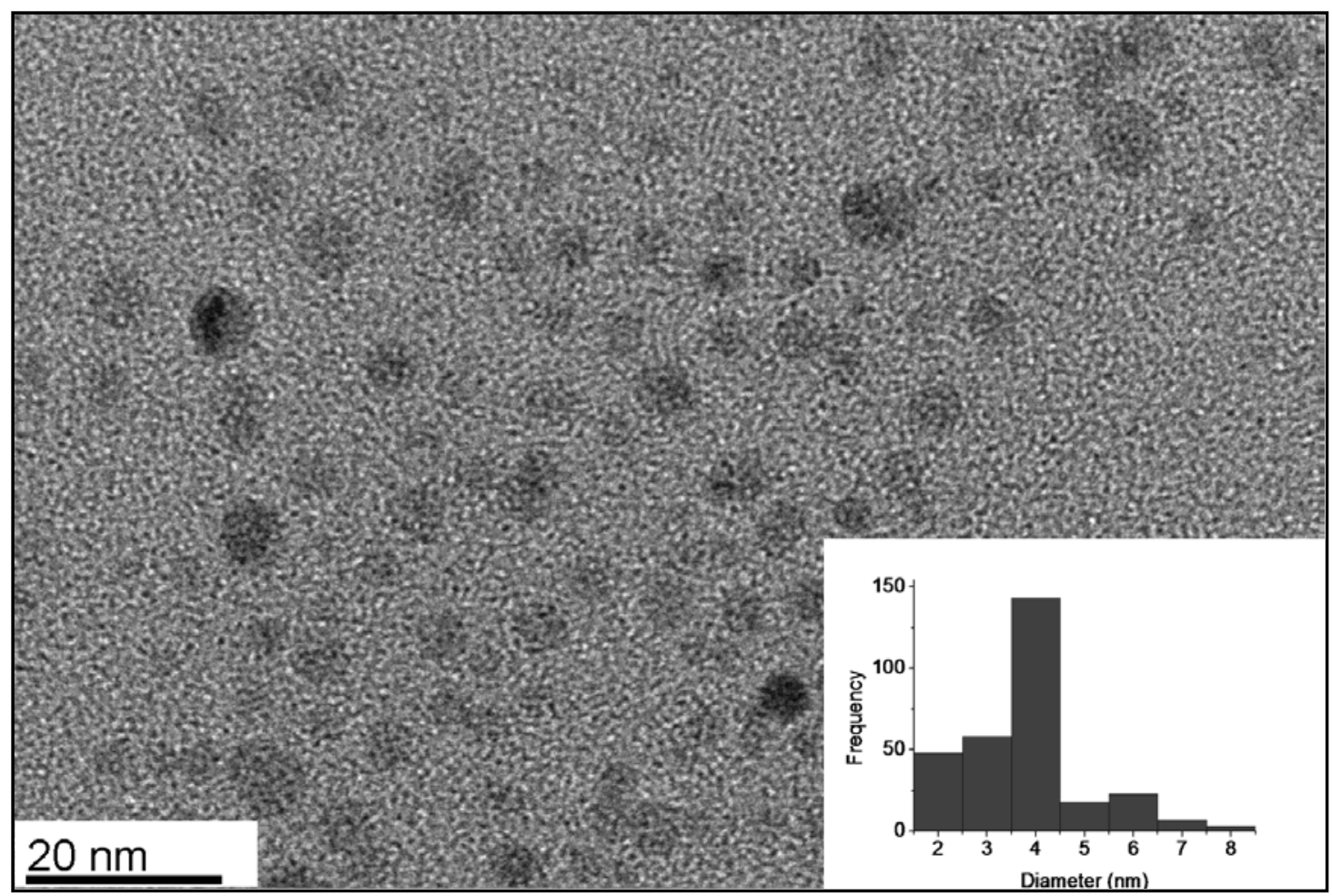

Figure 3-15: A low resolution TEM micrograph of the sample obtained from experiment 8

As may be seen in the

Figure 3-15, spherical nanoparticles were observed in the size range of $4.1 \pm 1.6 \mathrm{~nm}$.

Size distribution of the aggregates was relatively small compared to the sample

obtained from experiment 5 and 6 which indicates the effect of the size exclusion column chromatography. Most of the quantum dots were around the size of the silicon Bohr radius (4.8 nm). SAED could not be obtained, probably due to being light element and the small size of the quantum dots. The EDS spectroscopy showed the existence of silicon, however it showed the existence of side products containing bromine and carbon (data not shown). High resolution TEM micrograph could not be obtained probably because of these impurities. The average particle size was larger compared to the sample obtained from experiment 6 in which $\mathrm{SiBr}_{4}$ was used as a precursor.

The surface molecules of these nanoparticles was characterised by FTIR and ${ }^{1} \mathrm{H}$ NMR spectra. In 
Figure 3-16, peaks at $1250 \mathrm{~cm}^{-1}$ and $1460 \mathrm{~cm}^{-1}$ represent $\mathrm{Si}-\mathrm{CH}_{2}$ vibrational scissoring and symmetrical bending modes. The peak at $1108 \mathrm{~cm}^{-1}$ could possibly be assigned to C-N stretching deriving from the allylamine, however, the surfactant, $\mathrm{C}_{12} \mathrm{E}_{5}$, also has significant peak of C-O-C asymmetrical stretching at the same region and this peak is probably the overlap of those two stretches. The peak at $1635 \mathrm{~cm}^{-1}$ represents $\mathrm{N}-\mathrm{H}$ vibrational scissoring. Also N-H symmetrical and asymmetrical bending is seen in the peak at 3200-3650 $\mathrm{cm}^{-1}$. The peaks from $2855 \mathrm{~cm}^{-1}$ to $2924 \mathrm{~cm}^{-}$ ${ }^{1}$ represent $\mathrm{C}-\mathrm{CH}_{2}$ symmetrical and asymmetrical stretching.

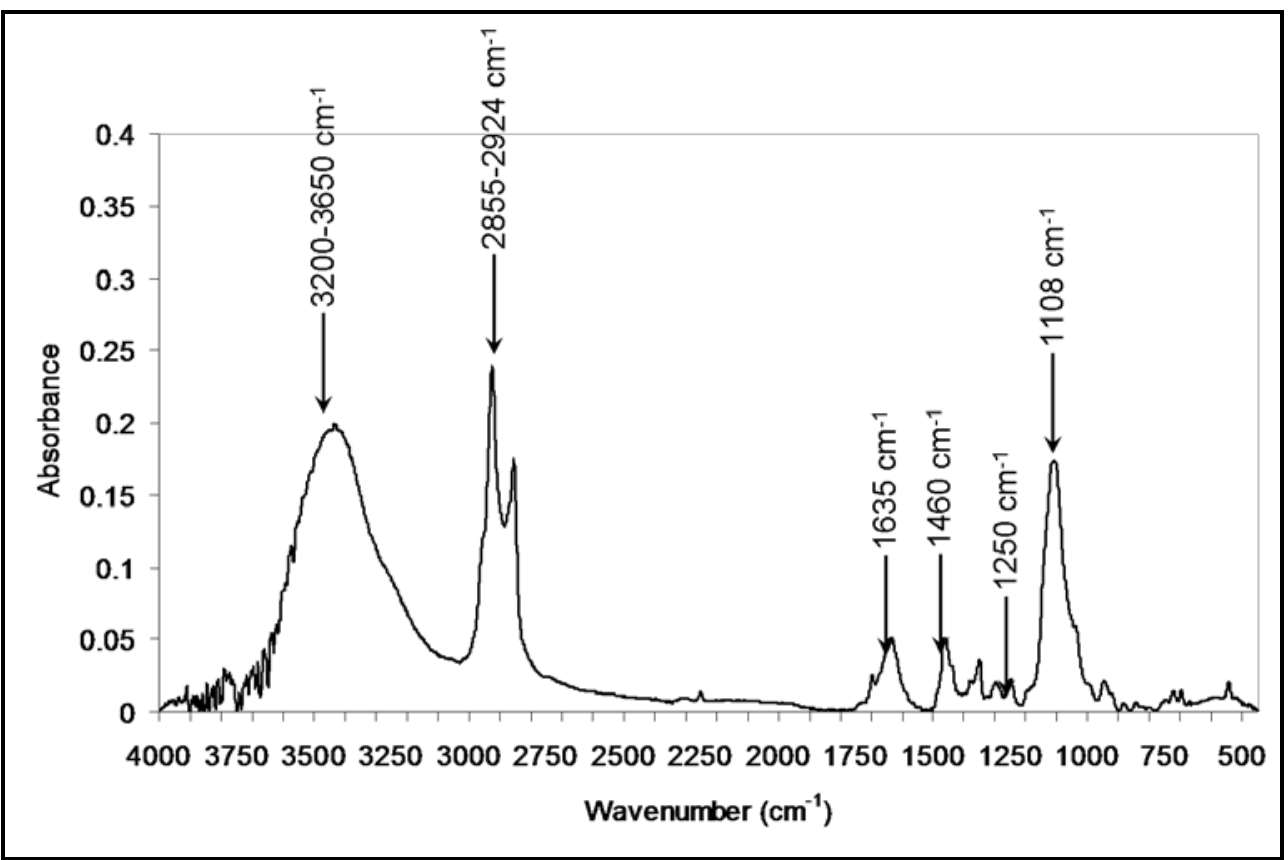

Figure 3-16: FTIR spectrum of the sample obtained from experiment 8 

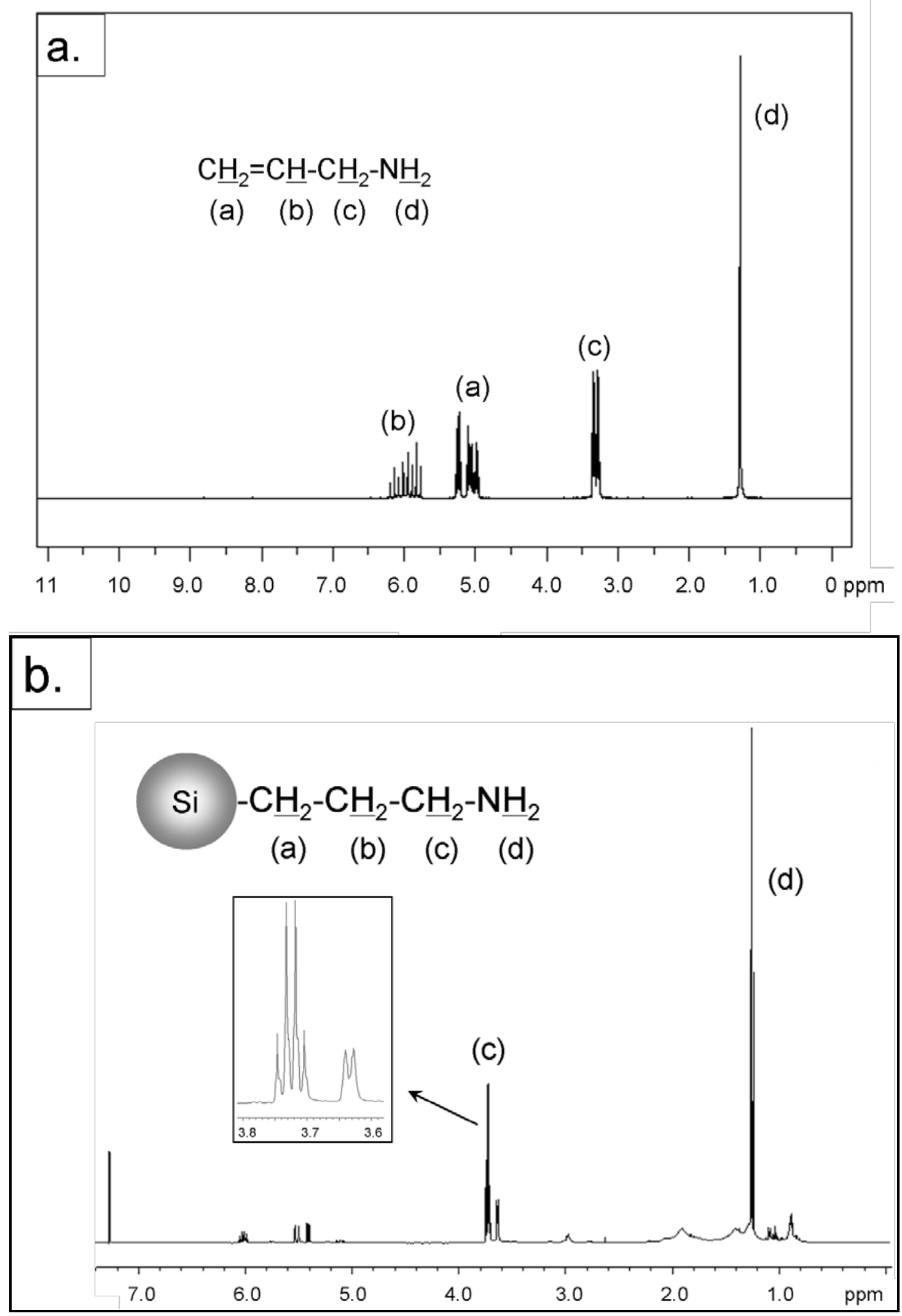

Figure 3-17: ${ }^{1} \mathrm{H}$ NMR spectrum of (a); free allylamine and (b); the sample obtained from experiment 8 
allylamine

${ }^{1} \mathrm{H}$ NMR (500MHz, $\left.\mathrm{CDCl}_{3}\right)$

$\delta: 1.29$ ppm (2H, s, H-(d)), $\delta: 3.31$ ppm (2H, d, H-(c), J=5.3 Hz), $\delta: 5.10$ ppm (2H, dd, H-(a) J=1.8, 4.3 Hz), $\delta: 5.95$ ppm (1H, m, H-(b))

Silicon quantum dots capped with allylamine

${ }^{1} \mathrm{H}$ NMR (500MHz, $\mathrm{CDCl}_{3}$ )

$\delta: 1.25$ ppm (2H, s, H-(d)), $\delta: 3.72 p p m(2 \mathrm{H}, \mathrm{dd}, \mathrm{H}-(\mathrm{c}), J=7.1,15.9 \mathrm{~Hz})$

The ${ }^{1} \mathrm{H}$ NMR spectrum showed a clear triplet at $1.25 \mathrm{ppm}$ representing protons attached to the nitrogen from the surface moiety of the silicon quantum dot (H-(d)). The quartet seen at $3.72 \mathrm{ppm}$ represents protons attached to the carbon next to the nitrogen (H-(c)). The signals indicating a double bond derived from allylamine can be seen at around 5.20 and $6.00 \mathrm{ppm}$. Since free allylamine should have been removed under vacuum it could be due to the attachment of nitrogen atom to the surface of silicon quantum dot. On the other hand the triplet seen at around $0.88 \mathrm{ppm}$ possibly indicates protons attached to the carbon next to silicon atom (H-(a)). Therefore the surface molecules of the silicon quantum dots could be a mixture of nitrogen atoms attached to the surface and carbon atoms attached to the surface.

\section{3-1-6. Silicon quantum dots capped with methyl 10- undecenoate: Experiment 9}

The purpose of the synthesis of silicon quantum dots capped with methyl 10undecenoate was to produce silicon quantum dots having an ester on the surface. Methyl 10-undecenoate has the ester structure combined of undecenoic acid and methanol. Since methyl group is not a good leaving group, the terminal end carbon double bond is expected to attach to the silicon quantum dot leaving the ester part at the terminal end of the capping agent. This ester group can react with an amine group to form an amide bond (scheme 3-1-6 (a)) which will broaden the possibilities of attaching bio-friendly molecules on the surface of the silicon quantum dots. Also the ester group can be converted to a carboxyl group using base (scheme 3-1-6 (b)), 
which may increase the dispersion of the silicon quantum dots under biological conditions.<smiles>C1=CCCCC=CC1</smiles>

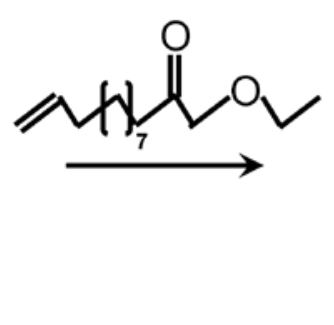

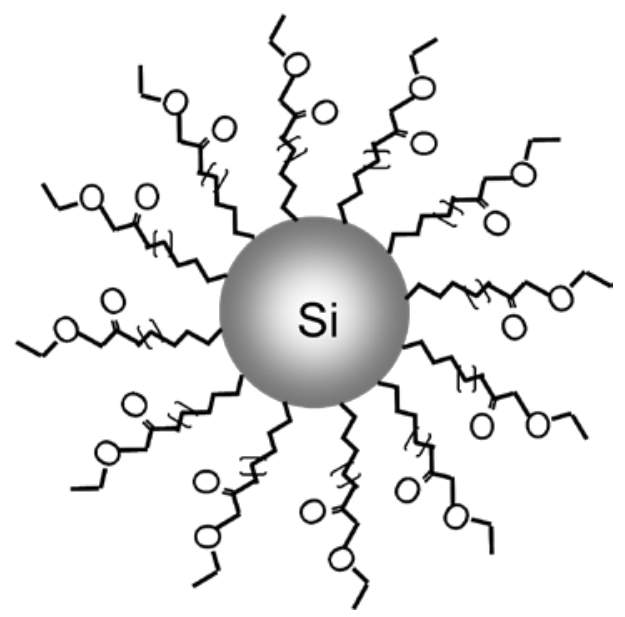

Scheme 3-1-6: Reaction scheme of methyl-10-undecenoate capping for silicon quantum dot

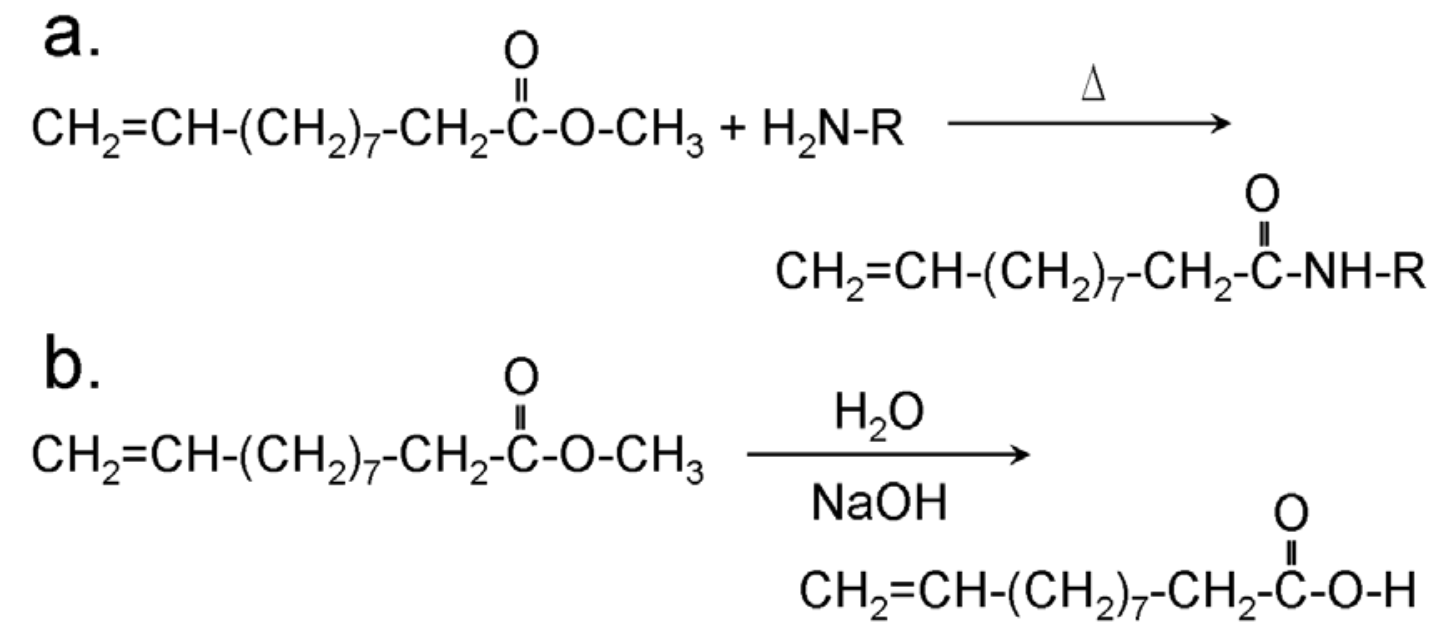

Scheme 3-1-7: Reaction scheme of ester (a); forming amide, and (b); forming carboxylic acid

\section{Experimental}

$0.25 \mathrm{~g}$ of pentaethylene glycol monododecyl ether $\left(\mathrm{C}_{12} \mathrm{E}_{5}\right), 0.3 \mathrm{~mL}(0.0026 \mathrm{~mol})$ of silicon tetrachloride $\left(\mathrm{SiCl}_{4}\right)$, and $4 \mathrm{~mL}$ of $2.0 \mathrm{M}$ lithium lithium borohydride () were used in this experiment. Surface passivation was conducted by UV-irradiation method with $5.0 \mathrm{~mL}$ of methyl 10-undecenoate for 4 hours. The white precipitate was removed by filtration and the solution was dried under vacuum then the yellow oil was re-dispersed in $10 \mathrm{~mL}$ of methanol. The precipitate was removed by filtration 
with millipore filter (0.45 $\mu$ m Durapore Membrane filter) and silicon quantum dots were purified by size exclusion column chromatography (LH20).

\section{Results}

TEM micrograph shows spherical nanoparticles. They are relatively mono disperse in size with diameter of $6.4 \pm 1.4 \mathrm{~nm}$. An SAED pattern from the same area matched to (111), (220), (311) and (400) patterns of crystalline silicon respectively. EDS spectrum showed the nanoparticles were mainly composed of silicon. The amount of silicon was $7.3 \%$. Also $3.3 \%$ of oxygen was detected. Carbon and oxygen peaks are possibly due to mixture of impurities and methyl 10-undecenoate attached on the surface. High resolution TEM image could not be obtained because of the impurities. These results imply the successful synthesis of crystalline silicon quantum dots. However in the FTIR spectrum and ${ }^{1} \mathrm{H}$ NMR spectrum, the dominant peaks are mainly from impurities such as the surfactant, $\mathrm{C}_{12} \mathrm{E}_{5}$. This could be due to the difficulties of separation by polarity. The ester parts of the methyl 10-undecenoate on the surface of the silicon quantum dots showed similar polarity to the ether groups of $\mathrm{C}_{12} \mathrm{E}_{5}$ and both molecules have non-polar parts in the form of alkyl chains. These similarities allowed a relatively large concentration of the surfactant through column. Also this implies that there was definitely an interaction between ester groups and ether groups and it possibly made the size separation more difficult. 


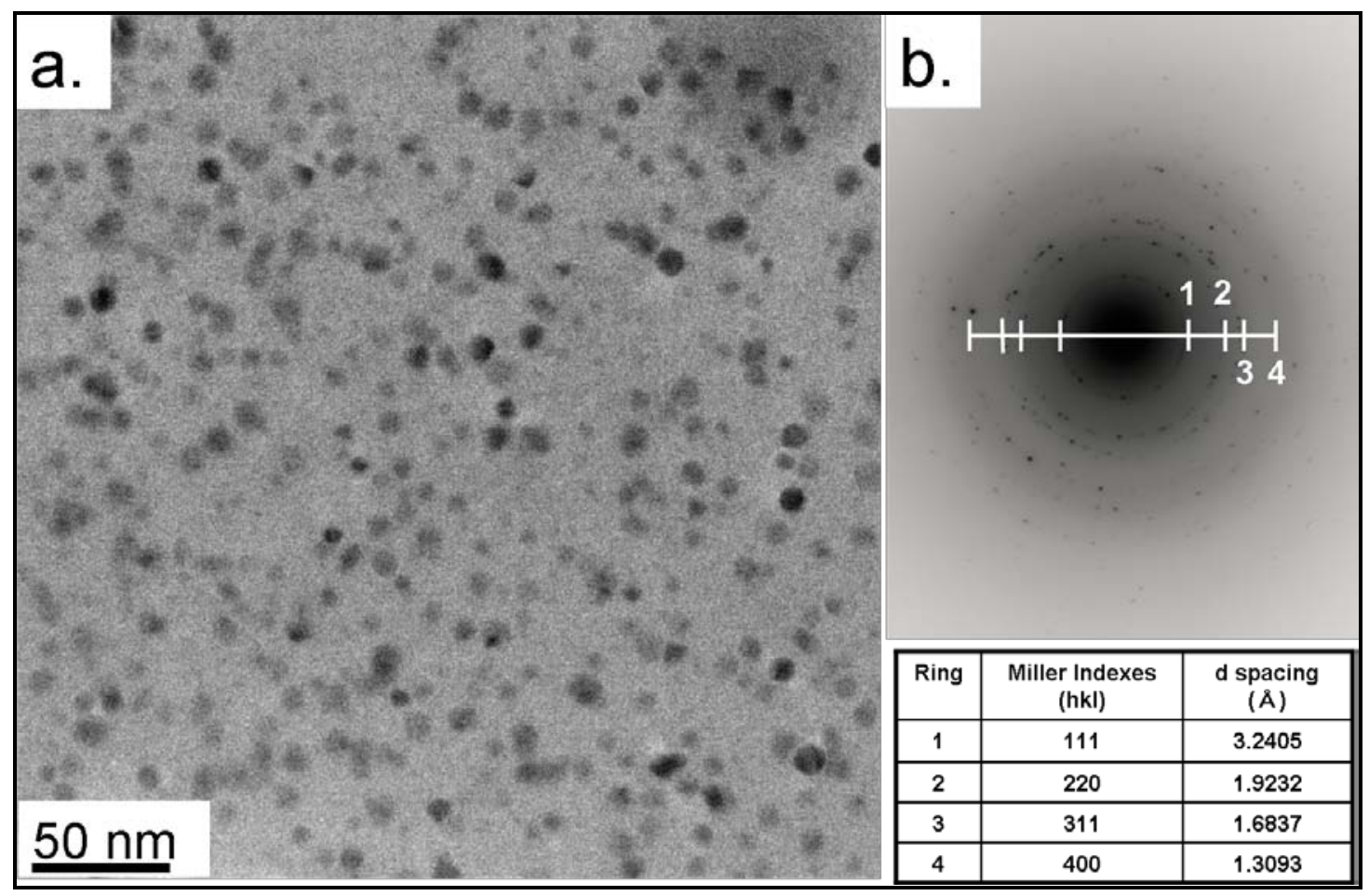

Figure 3-18: (a) a TEM micrograph of silicon quantum dots capped with methyl 10undecenoate; (b) SAED pattern correspondent to nanoparticles shown in (a); (c) ESD spectrum correspondent to nanoparticles shown in (a); (d) size distribution graph 

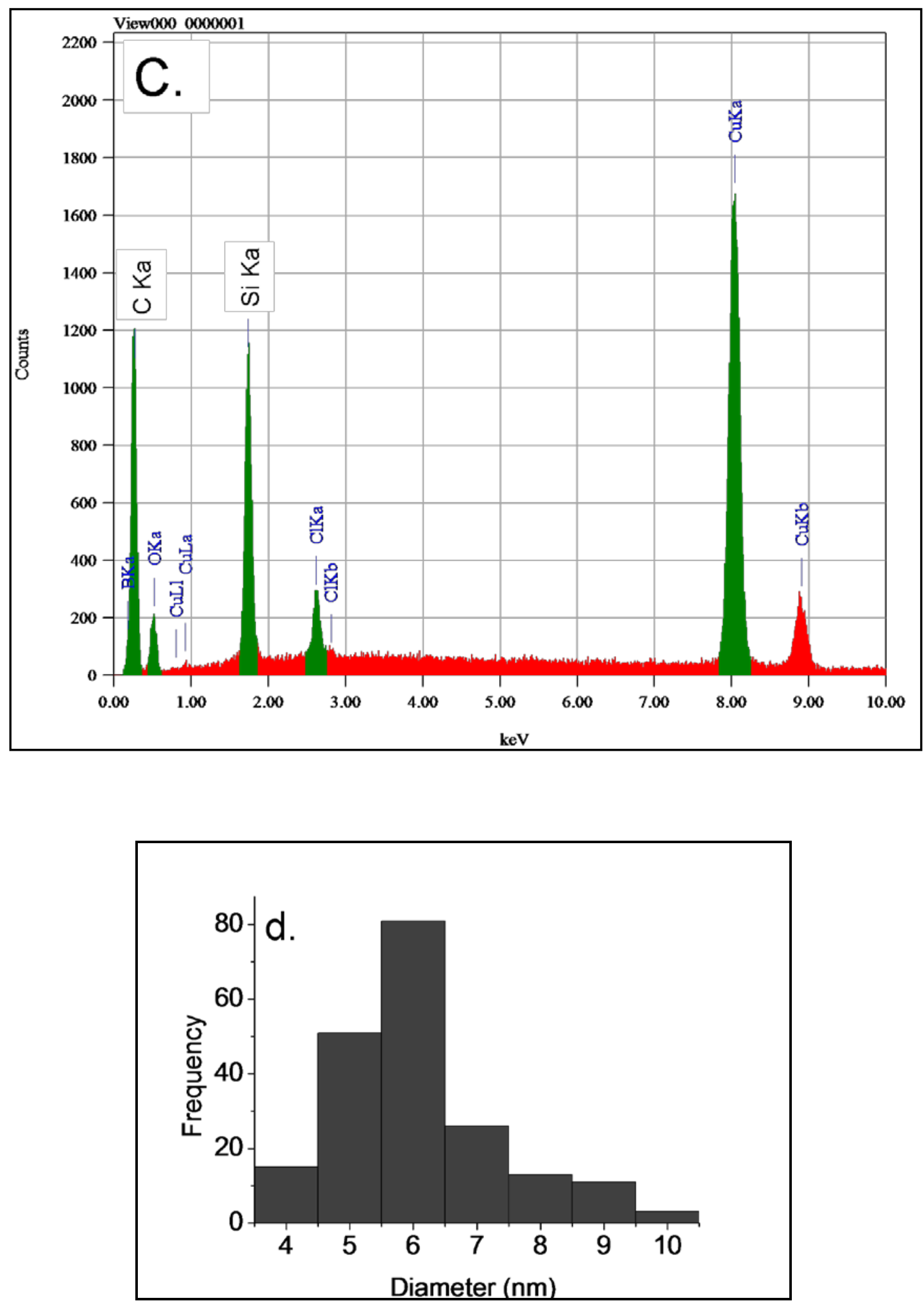

Figure 3-18: (a) a TEM micrograph of silicon quantum dots capped with methyl 10undecenoate; (b) SAED pattern correspondent to nanoparticles shown in (a); (c) ESD spectrum correspondent to nanoparticles shown in (a); (d) size distribution graph 


\section{3-1-7. Silicon quantum dots capped with limonene: Experiment 10}

Limonene was chosen as capping molecules for silicon quantum dot because of its relatively simple chemical structure compared to many other drug molecules. Limonene is contained in citrus plants such as lemon or orange in high concentration and is the substance that provides the citrus smell. It is also proven that limonene has an anti-tumour effect. Limonene is believed to encourage the growth of phase II detoxicating enzyme which prohibits initiation of cancerogenesis. ${ }^{34}$ The purpose of this experiment is to attach drug molecules directly onto the surface of the silicon quantum dots for drug delivery applications. One of the carbon-carbon double bonds (probably terminal one since the other one has more steric hindrance) is expected to form covalent bond with siliton atom on the surface of the silicon quantum dot.

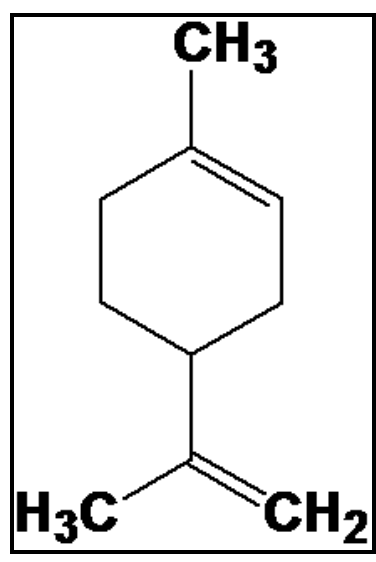

Figure 3-19: Structure of limonene 

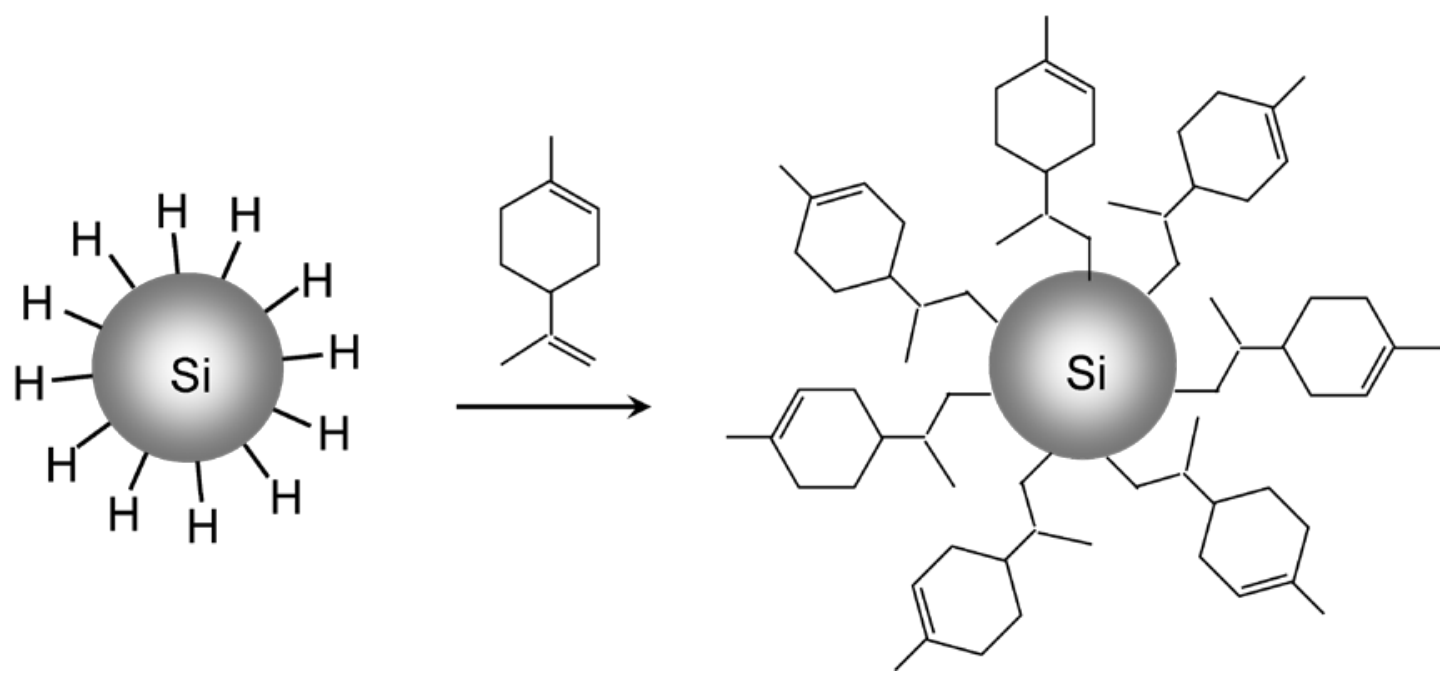

Scheme 3-1-8: Reaction scheme of limonene capping for silicon quanutm dot

\section{Experimental}

$0.25 \mathrm{~g}$ of pentaethylene glycol monododecyl ether $\left(\mathrm{C}_{12} \mathrm{E}_{5}\right), 0.3 \mathrm{~mL}(0.0026 \mathrm{~mol})$ of silicon tetrabromide $\left(\mathrm{SiBr}_{4}\right)$, and $4 \mathrm{~mL}$ of $1.0 \mathrm{M}$ lithium aluminiumhydride were used in this experiment. Surface passivation was conducted by UV-irradiation method with $4 \mathrm{~mL}$ of limonene for 4 hours. $\mathrm{N}$-methylformamide was added to the reaction mixture in a separation funnel and the silicon quantum dots were extracted by liquid-phase separation. The hexane layer was collected and dried under vacuum then the yellow oil was re-dispersed in anhydrous hexane. 


\section{Results}

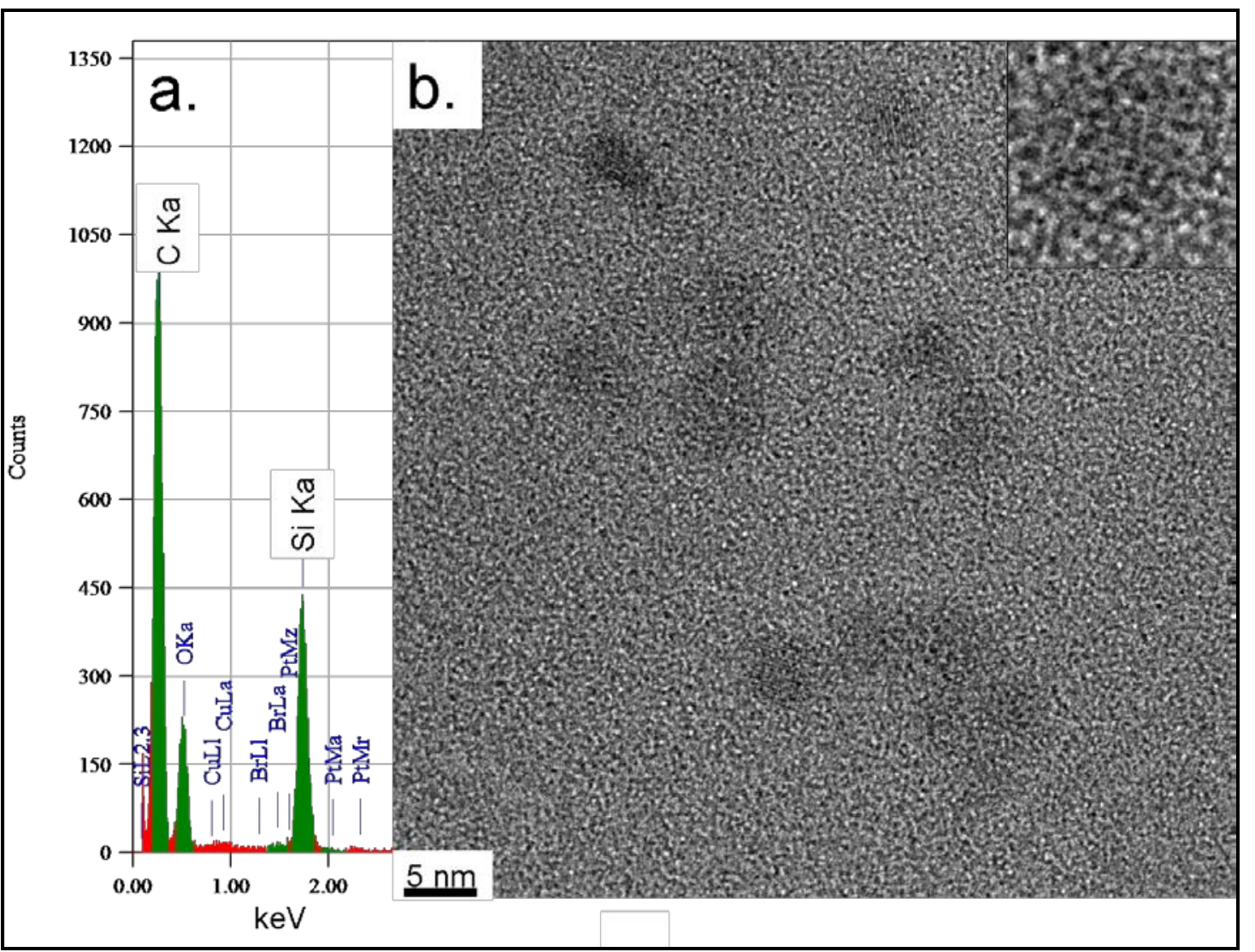

Figure 3-19: (a): EDS spectrum of the sample obtained from experiment 10, (b): a TEM micrograph of the same sample

Some particles were observed by TEM. The shapes of those particles were almost spherical with a size range of $4.7 \pm 0.5 \mathrm{~nm}$ (Figure 3-20). Very subtle lattice fringes were observed from the aggregates however no SAED was obtained. From EDS analysis, existence of silicon was confirmed. The atomic \% of silicon was $4.2 \%$ and only $0.2 \%$ of bromine was detected. No aluminium peak was observed, which indicates that the aggregates are mainly composed of silicon.

Figure 3-21 shows ${ }^{1} \mathrm{H}$ NMR spectra of free limonene and silicon quantum dots after reaction with limonene. In the spectrum of the silicon quantum dots sample two new signals at $0.89 \mathrm{ppm}$ and $1.27 \mathrm{ppm}$ were observed which did not appear in the free limonene spectrum. This result indicates that one of the double bonds of limonene was attached to the silicon quantum dots. The signals corresponding to $\mathrm{H}-(\mathrm{h})$ and $\mathrm{H}-$ (b) at $4.71 \mathrm{ppm}$ and $5.50 \mathrm{ppm}$ from the free limonene were still observed in the spectrum of the silicon quantum dot sample which suggests there are remaining free limonene molecules in the sample. In the spectrum of free limonene, those two signals 
integrate for two and one protons respectively which matches to the structure of limonene however in the spectrum of the silicon quantum dots, the integral of these protons did not show a $2: 1$ ratio but $1.5: 1$ ratio. This result indicates that external double bonds of some of the limonene molecules are attached to the surface of silicon quantum dots and the signal appears at $0.89 \mathrm{ppm}$ which shifted to up field. This could be due to the less steric hindrance of the external double bond compared to the internal one of the ring.

Limonene

${ }^{1} \mathrm{H}$ NMR (500MHz, $\left.\mathrm{CDCl}_{3}\right)$

$\delta: 1.50$ ppm (2H, dddd, H-(e), J=13.4, 10.1, 10.0, $3.9 \mathrm{~Hz}), \delta: 1.67 \mathrm{ppm}(3 \mathrm{H}, \mathrm{s}, \mathrm{H}-$ (a)), $\delta: 1.75$ ppm (3H, s, H-(g)), $\delta: 1.81$ ppm (1H, m, H-(d)), $\delta: 1.92$ ppm (1H, ddd, H-(c), $J=15.0,10.0,7.7 \mathrm{~Hz}), \quad \delta: 1.98 \mathrm{ppm}(1 \mathrm{H}, \mathrm{ddd}, \mathrm{H}-(\mathrm{f}), J=14.3,10.0,4.0 \mathrm{~Hz}), \quad \delta$ : 1.99 ppm (1H, ddd, H-(f), J=14.3, 3.9, 1.9 Hz), $\delta: 2.07$ ppm (1H, ddd, H-(c), J=15.0, 5.2, $3.9 \mathrm{~Hz}), \delta: 4.72 \mathrm{ppm}(2 \mathrm{H}, \mathrm{d}, \mathrm{H}-(\mathrm{h}), J=1.3 \mathrm{~Hz}), \delta: 5.41 \mathrm{ppm}(1 \mathrm{H}, \mathrm{dd}, \mathrm{H}-(\mathrm{b})$, $J=7.7,5.2 \mathrm{~Hz})$

Silicon quantum dots capped with limonene

${ }^{1} \mathrm{H}$ NMR (500MHz, $\mathrm{CDCl}_{3}$ )

$\delta: 0.89 \mathrm{ppm}(2 \mathrm{H}, \mathrm{s}, \mathrm{H}-(\mathrm{i})), \delta: 1.27 \mathrm{pm}(2 \mathrm{H}, \mathrm{m}, \mathrm{H}-(\mathrm{h})), \delta: 1.50 \mathrm{ppm}$ (2H, dddd, H(e), J=5.6, 5.9, 5.7, $5.6 \mathrm{~Hz}$ ), $\delta: 1.66 \mathrm{ppm}$ (3H, s, H-(a)), $\delta: 1.74 \mathrm{ppm}$ (3H, s, H-(g)), $\delta: 1.79$ ppm (1H, d, H-(d), J=12.4 Hz), $\delta: 1.94$ ppm (2H, d, H-(c),(f), $J=16.8 \mathrm{~Hz})$, $\delta: 2.06$ ppm (2H, dd, H-(c),(f), $J=16.1,18.5 \mathrm{~Hz}), \delta: 5.41$ ppm (1H, d, H-(b), $J=0.7$ $\mathrm{Hz})$ 

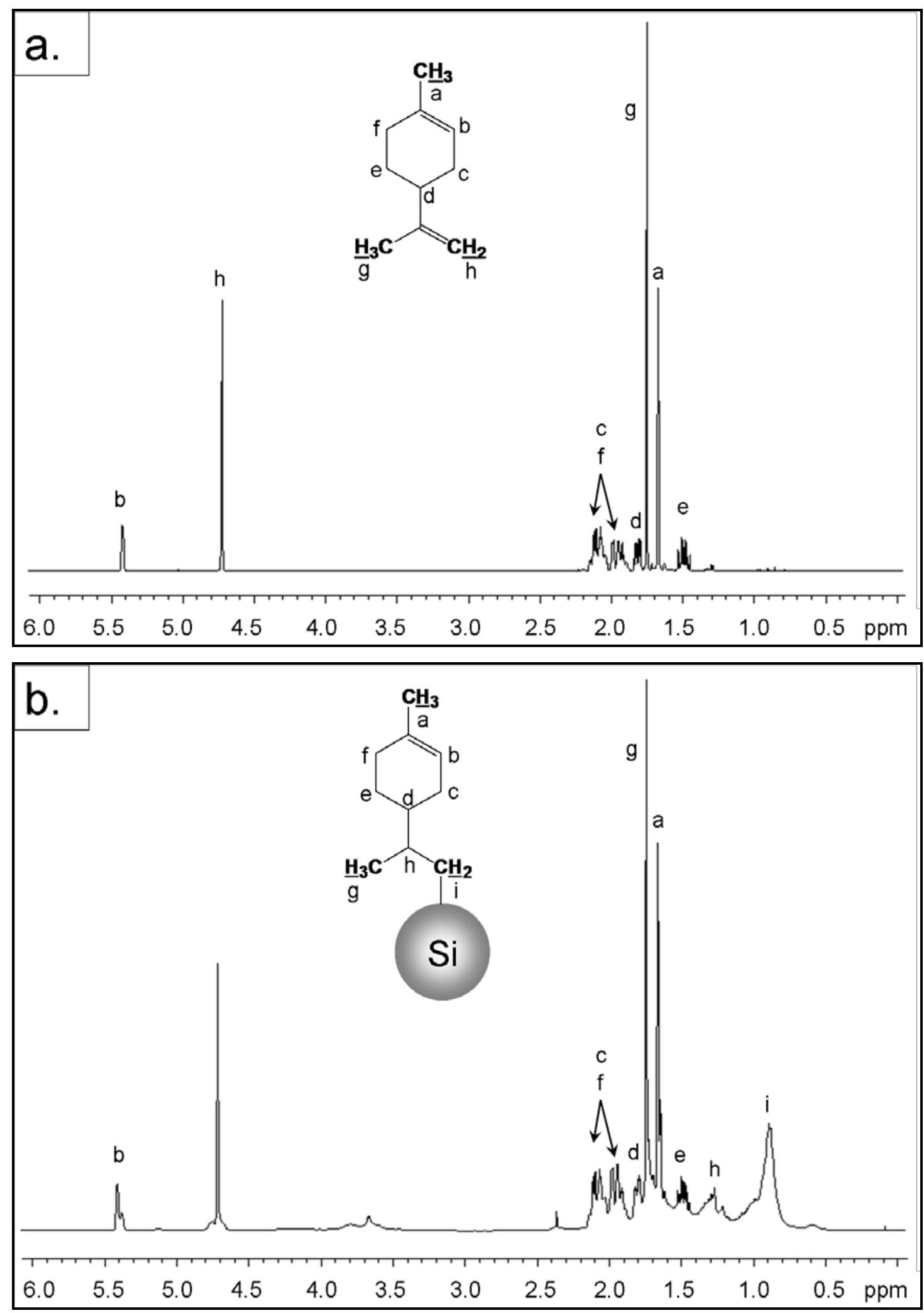

Figure 3-20: (a); ${ }^{1} \mathrm{H}$ NMR spectrum of free limonene, (b); ${ }^{1} \mathrm{H}$ NMR spectrum of the sample obtained from experiment 8 


\section{3-1-8. Silicon quantum dots capped with propargylamine: Experiment 11}

Propargylamine has a terminal carbon triple bond and amine group on the other end. Since it has terminal riple bond it was expected to form covalent bonds with two carbon atoms with silicon atom on the surface of the silicon quantum dot. It can reduce the steric hinderance of the capping molecuels which enables the better capping efficiency on the surface. Also as allylamine capping, the terminal amine group can react with other functional groups to attach biological molecules to the quanutm dots.<smiles></smiles>
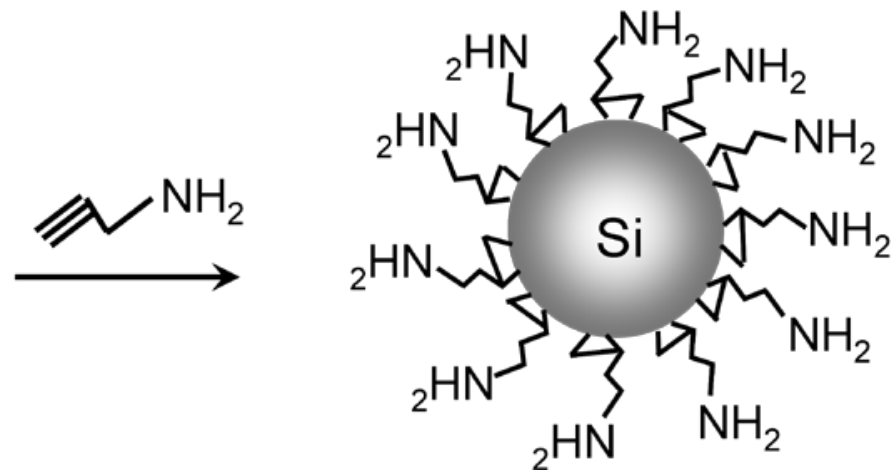

Scheme 3-1-9: Reaction scheme of propagylamine capping for silicon quanutm dot

\section{Experimental}

$0.25 \mathrm{~g}$ of pentaethylene glycol monododecyl ether $\left(\mathrm{C}_{12} \mathrm{E}_{5}\right)$, was dissolved in $50 \mathrm{~mL}$ of anhydrous hexane and $0.3 \mathrm{~mL}(0.0026 \mathrm{~mol})$ of $\left(\mathrm{SiCl}_{4}\right)$, and $4 \mathrm{~mL}$ of $2.0 \mathrm{M}$ lithium borohydride were used in this experiment. Surface passivation was conducted by UVirradiation method with $1.5 \mathrm{~mL}$ of propargylamine for 4 hours. White and pale yellow precipitate was observed in the bottom of the vessel. The precipitate was removed by filtration and a pale yellow solution was obtained. Solvent was removed under vacuum. A yellow oil which fluoresces strongly blue was obtained and re-dissolved in methanol.

\section{Results}

This sample was characterized only by ${ }^{1} \mathrm{H}$ NMR. The NMR spectra of free propargylamine and after attachment to silicon quantum dots are shown in Figure 3-22 (a), (b). 

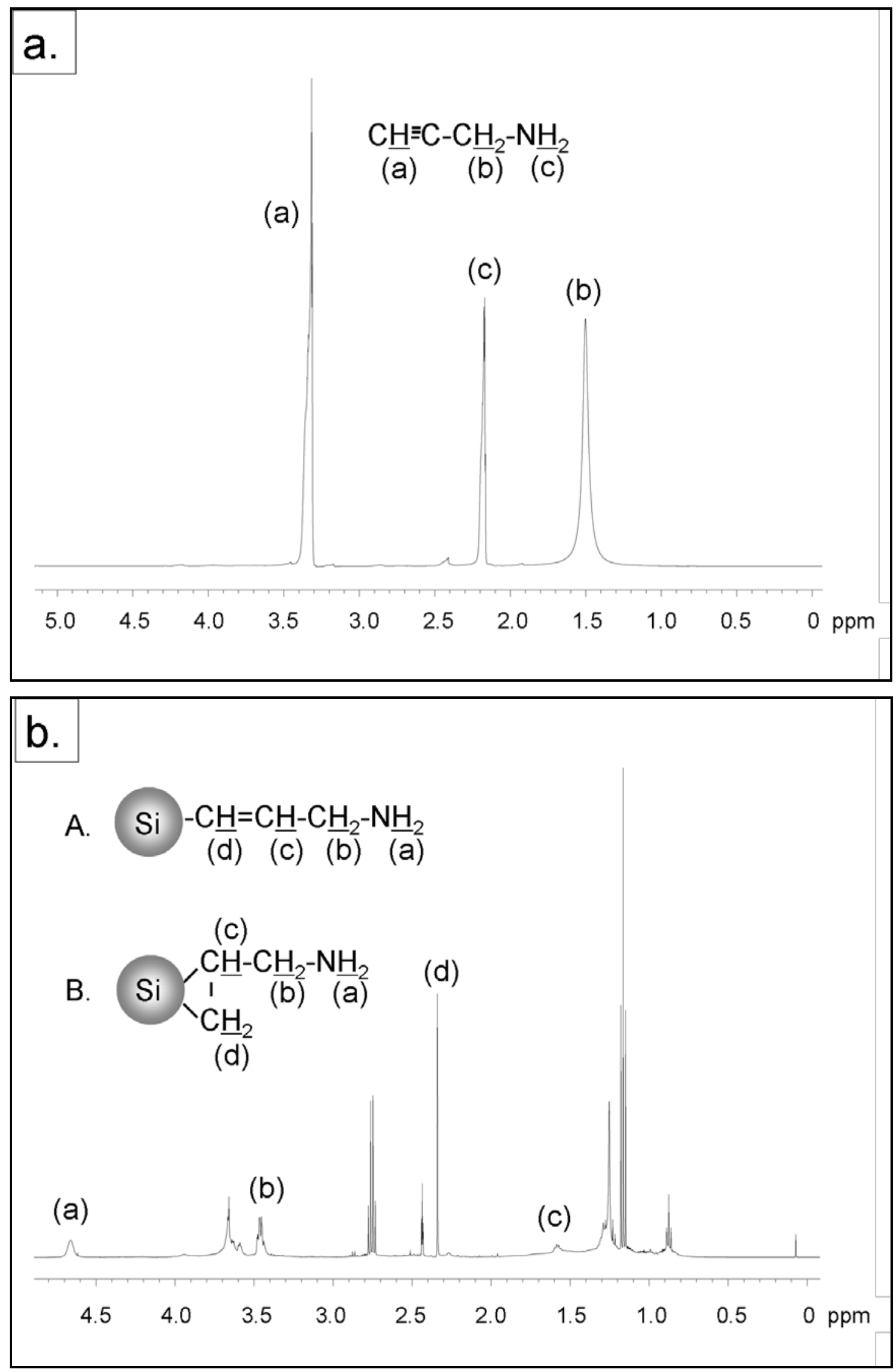

Figure 3-21: (a); ${ }^{1} \mathrm{H}$ NMR spectrum of free propargylamine, (b); ${ }^{1} \mathrm{H}$ NMR spectrum of the sample obtained from experiment 11 
In the spectrum of free propargylamine, three clear signals can be observed. The one at 3.35 ppm corresponds to two protons from the amine group. The signals at 2.18 ppm and 1.49 ppm correspond to $\mathrm{H}-(\mathrm{c})$ and $\mathrm{H}-(\mathrm{b})$ respectively. However those signals cannot be seen in the spectrum from the sample. From g-COSY analysis, the protons corresponding to the signals at $4.68 \mathrm{ppm}$ and $3.46 \mathrm{ppm}$ are spin-spin coupled. Also the proton at $3.46 \mathrm{ppm}$ is spin coupled to the proton at $2.32 \mathrm{ppm}$ and this proton (at $2.32 \mathrm{ppm}$ ) is spin coupled to the one at $1.59 \mathrm{ppm}$. From the result, the signals at $4.68 \mathrm{ppm}$ and $3.46 \mathrm{ppm}$ probably correspond to $\mathrm{H}-(\mathrm{a})$ and $\mathrm{H}-(\mathrm{b})$ respectively. The signals at $2.32 \mathrm{ppm}$ and $1.59 \mathrm{ppm}$ integrate for two protons and one proton respectively therefore those peaks possibly correspond to $\mathrm{H}-(\mathrm{d})$ and $\mathrm{H}$-(c) from structure B respectively.

Propargylamine

${ }^{1} \mathrm{H}$ NMR (500MHz, $\left.\mathrm{CDCl}_{3}\right)$

$\delta: 1.49$ ppm (2H, s, H-(b)), $\delta: 2.18$ ppm (2H, s, H-(c)), $\delta: 3.35$ ppm (1H, s, H-(a))

Silicon quantum dots capped with propargylamine

${ }^{1} \mathrm{H}$ NMR (500MHz, $\mathrm{CDCl}_{3}$ )

$\delta: 1.59$ ppm (1H, m, H-(c)), $\delta: 2.32$ pm (2H, s, H-(d)), $\delta: 3.46 \mathrm{ppm}(2 \mathrm{H}, \mathrm{dd}, \mathrm{H}-(\mathrm{b})$,

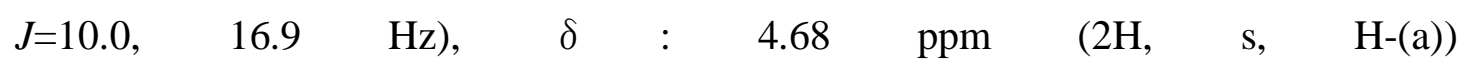

\section{3-2. Purification}

\section{3-2-1. Liquid-phase separation}

Liquid-phase separation conducted to purify the silicon quantum dots capped with 1,9-decadiene by the Pt catalyst was monitored by ${ }^{1} \mathrm{H}$ NMR spectroscopy. The purpose of the purification is to remove surfactant which might be toxic to organs. ${ }^{1} \mathrm{H}$ NMR spectrum was taken after each wash cycle. The silicon quantum dots were washed with $\mathrm{N}$-methylformamide which readily solubilises the surfactant ${ }^{35}$ and extracted into hexane layer. The results of silicon quantum dots capped with 1,9decadinene were shown in Figure 3-23 (a) to (d). 


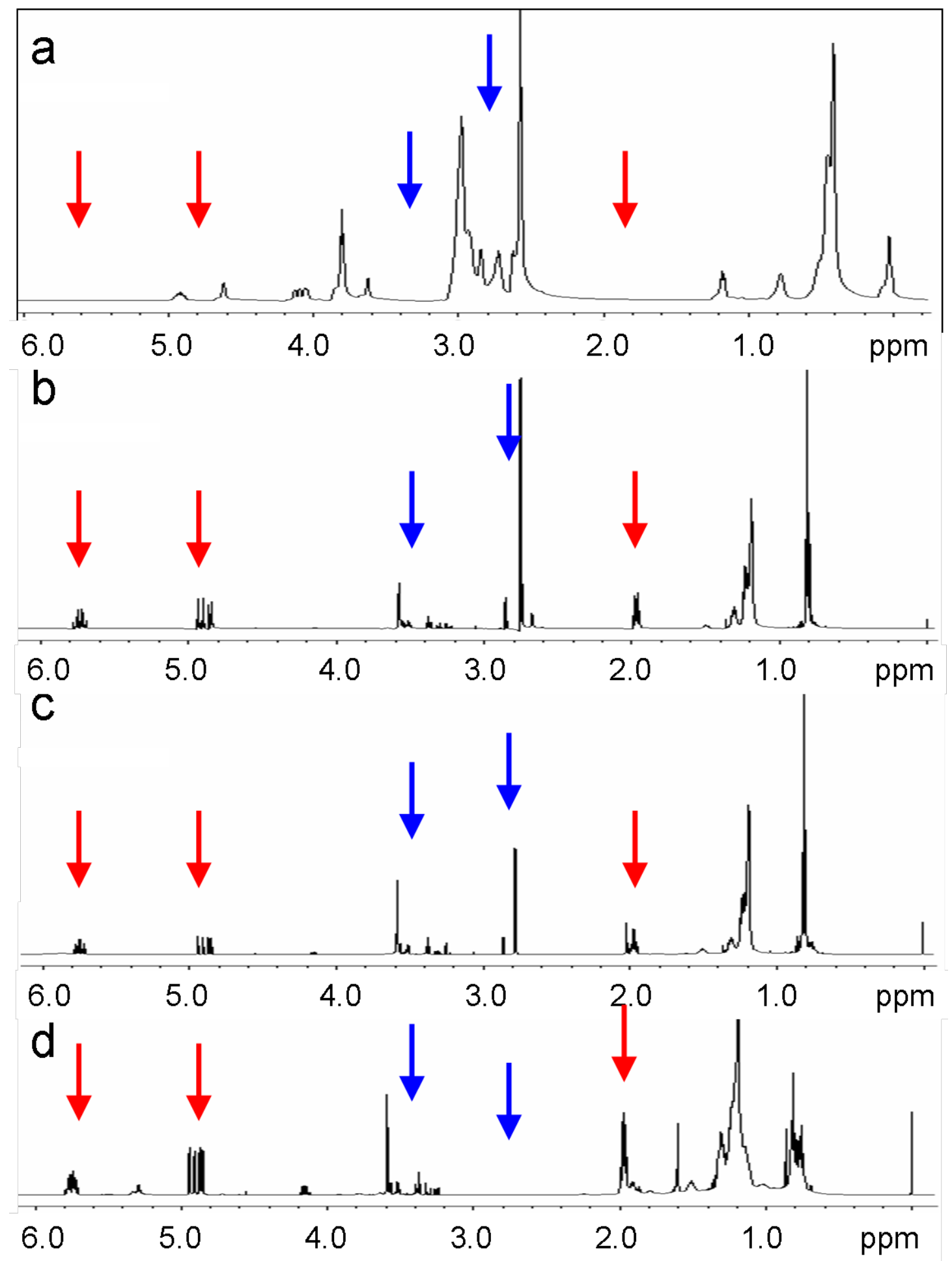

Figure 3-22: ${ }^{1} \mathrm{H}$ NMR spectra of the silicon quantum dots capped with 1,9-decadiene washed with $\mathrm{N}$-methylformamide, (a); 0 time, (b); 1 time, (c); 2 times and (d); 3 times 
As may be seen in the NMR spectra, each time after wash certain amount of surfactant was removed. The integrals of the ether parts of surfactant (blue arrow) and double bond of decadiene (red arrow) were used to calculate the relative amount of the surfactant and the Table 3-1of the calculations was shown below. In each wash about $50 \%$ of the surfactant present was removed. After 3 washes only $12 \%$ of the initial surfactant was left on the sample, indicating the success of liquid-phase separation.

Table 3-1: Calculations of remaining surfactant from liquid-phase separation of the silicon quantum dots capped with 1,9-decadiene

\begin{tabular}{|l|l|l|l|}
\hline Wash & Intensity $\left(\mathrm{C}_{12} \mathrm{E}_{5}\right)$ & Intensity (double bond) & Remained surfactant (\%) \\
\hline 0 & 41.30 & 100 & 100 \\
\hline 1 & 16.26 & 100 & 39.37 \\
\hline 2 & 11.83 & 100 & 28.66 \\
\hline 3 & 4.97 & 100 & 12.03 \\
\hline
\end{tabular}

The silicon quantum dots capped with 1-hexene were also purified by liquid-phase separation with $\mathrm{N}$-methylformamide. The sample was washed 5 times with $\mathrm{N}$ methylformamide and washed with water to remove $\mathrm{N}$-methylformamide. The ${ }^{1} \mathrm{H}$ NMR spectra of 0 wash and 5 washes are shown in Figure 3-24. 


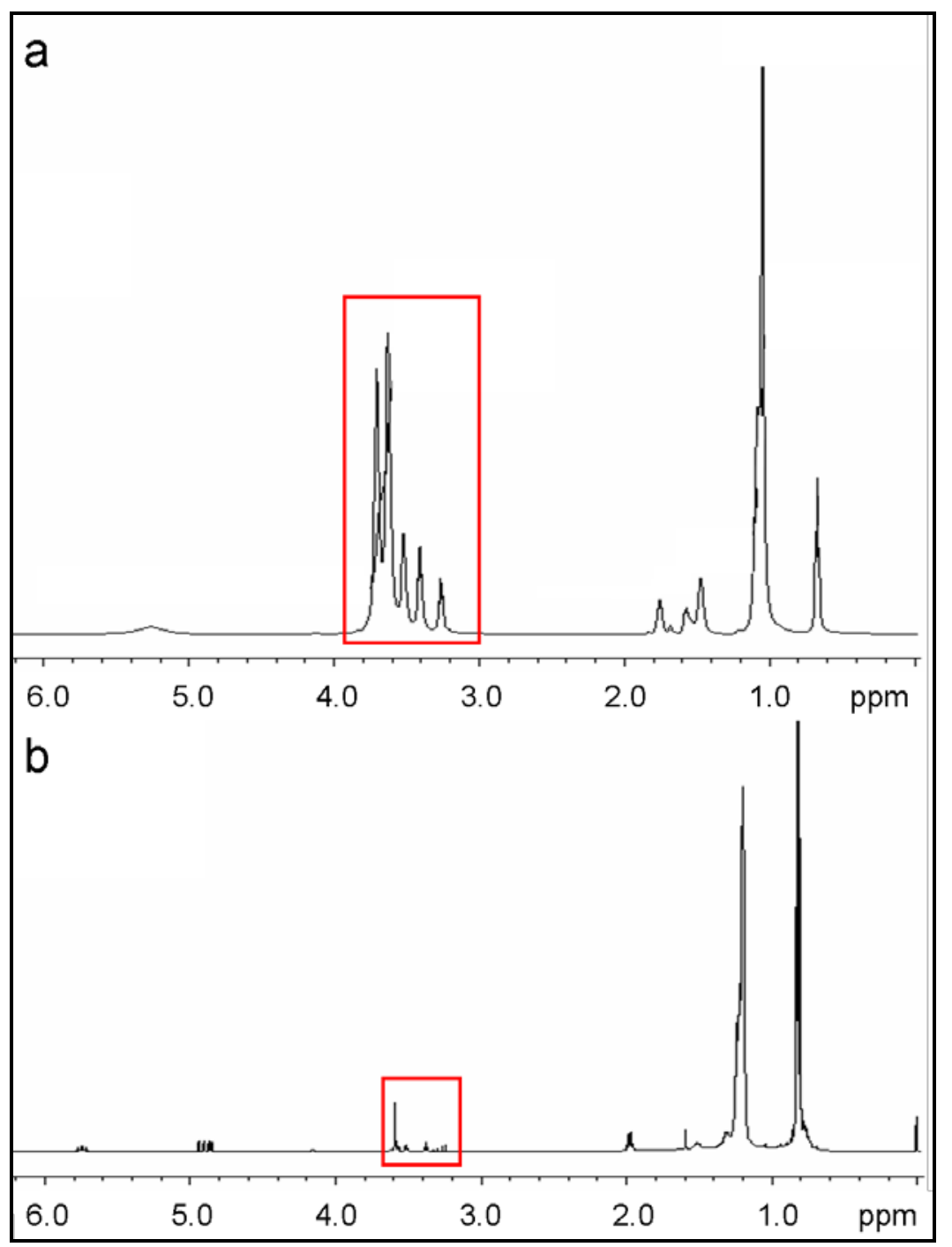

Figure 3-23: ${ }^{1} \mathrm{H}$ NMR spectra of the silicon quantum dots capped with 1-hexene after washed with $\mathrm{N}$-methylformamide (a); 0 time and (b); 5 times

As may be seen in Figure 3-24, significant amount of surfactant was removed after 5 washes even though the signal from the silicon quantum dots remained almost the same. According to the same calculation as above, only $0.86 \%$ of the initial surfactant remained after 5 washes which means almost $100 \%$ of the surfactant was removed by liquid-phase separation.

The product with 0,1 , and 2 wash did not show any particles on TEM analysis because of the impurities. After 3 washes, spherical nanoparticles with lattice fringes were observed. However after more than 5 wash, hardly any nanoparticles were observed from TEM. It could be due to the loss of the nanoparticles during the purification process. 


\section{3-2-2. Size exclusion column chromatography}

Size exclusion column chromatography was applied to purify silicon quantum dots capped with 1,5-hexadiene. Since surface molecules of the silicon quantum dots were quite similar to the capping molecules it is difficult to remove an excess of free capping molecules by applying affinity or ion exchange chromatography. Therefore we decided to use the size difference between free capping molecules and silicon quantum dots and applied size exclusion chromatography. There are very few reports on purification of quantum dots especially column chromatography. Colvin et. al ${ }^{36}$ reported the purification of CdSe quantum dots by size exclusion chromatography. However, to the best of our knowledge, there is no report of column chromatography purification of silicon quantum dots. LH20 sephadex gel was chosen as the packing material because of its flexibility of mobile phase. In contrast to other packing materials, methanol as polar solvent and toluene as non-polar solvent can be used for this packing material which gives much wider options for capping molecules for the silicon quantum dots. The result of the column chromatography on the silicon quantum dots capped with 1,5-hexadiene was shown in Figure 3-25. 


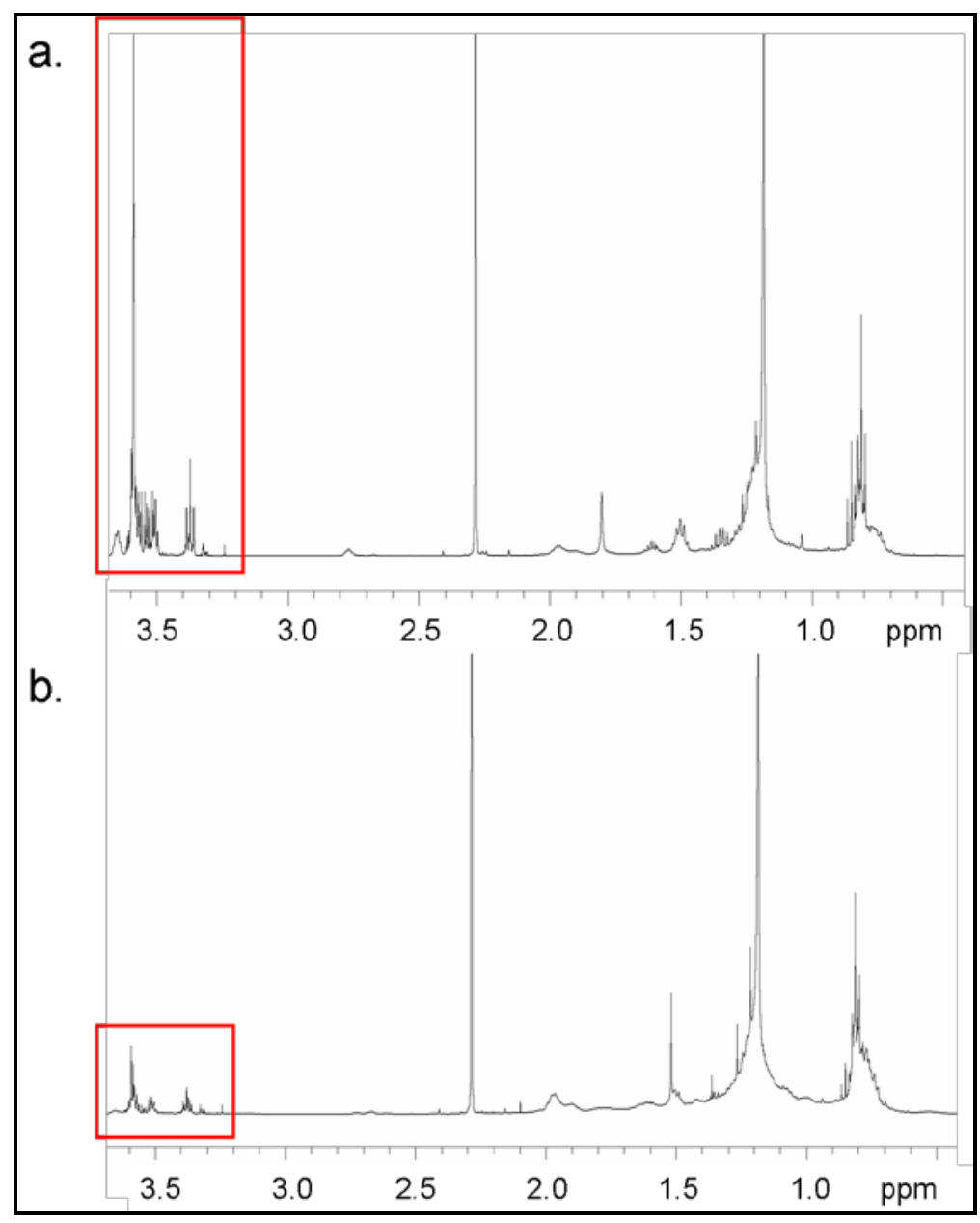

Figure 3-24: ${ }^{1} \mathrm{H}$ NMR spectra of the silicon quantum dots capped with 1,5-hexadiene (a); before size exclusion column chromatography and (b); after

As may be seen in the NMR spectra, the crude sample and purified sample showed a significant difference on the purity. After only one pass through the column chromatography $70 \%$ of surfactant was removed. On the other hand almost $100 \%$ of the silicon quantum dots remained in the solution. The success of this column chromatography purification method for the silicon quantum dots has led to a decrease in the common problems encountered for their biocompatibility. However, this column chromatography was not shown to be successful in removing TOAB. It is probably because the shape of the surfactant affects the separation mechanism of the packing materials. The packing material has its pore size around 25-100 $\mu$ m however inside the bead there are channels to trap the molecuels and those shapes can affect the ability of trapping the molecules depending on their shape as well. 


\section{3-3. Discussion and Summary}

From the results above it was confirmed that microemulsion synthesis can produce relatively mono dispersed silicon quantum dots at room temperature. Different sizes of nanoparticles were produced by changing the surfactant. The results from experiment 2 and 3, by using TOAB as a surfactant produced more than $1.0 \mathrm{~nm}$ smaller size nanoparticles compared to $\mathrm{C}_{12} \mathrm{E}_{5}$. Size distribution of the nanoparticles was also more uniform by $0.6 \mathrm{~nm}$ when $\mathrm{TOAB}$ was used compared to $\mathrm{C}_{12} \mathrm{E}_{5}$. It is possibly due to the size of micelles. The molar interfacial area of the cationic surfactant at the surfactant to oil boundary is bigger than to non-ionic surfactant, and as result the radius of micelle is lower than that of another surfactant under the same conditions. $^{37}$

From all the experiments described above, it was confirmed that both the platinum catalyst capping and the UV-irradiation capping can produce silicon quantum dots. However in the results from 1,5-hexadiene capping by the Pt catalyst (experiment 5) the spherical nanoparticles seen in TEM micrograph could not be confirmed as crystalline silicon. No nanoparticles could be observed from allylamine capping by the Pt catalyst (experiment 7) either. By comparison, from all the experiments of the UV-irradiation capping showed spherical silicon nanoparticles. Therefore we can conclude that the UV-irradiation capping would be more efficient system compared to the platinum catalyst system. Also from the results of experiment 5 and 6, a smaller particle size distribution in the UV-irradiation method was confirmed compared to the platinum catalyst capping. This could be due to the formation of platinum nanoparticles which can be larger size than silicon quantum dots. Since some of the precursor can be lost as silane gas when it is reduced ${ }^{32,33}$, if extra reducing agent was left platinum catalyst will be reduced to form platinum nanoparticles. UV-irradiation capping can also prevent producing platinum nanoparticle.

Experiment 1 showed the failure of the capping method using a molecule containing an alcohol group. Since the oxygen atom of the hydroxyl group has two lone pairs of electrons and has high electron negativity, the hydroxyl group can be attracted by the surface of silicon quantum dot. If deprotonation occurred, the oxygen atom can easily 
form a covalent bond with the silicon atom on the surface. The success of allylamine capping from experiment 8 showed that silicon did not form a bond with the nitrogen atom even though it also has a lone pair electrons. This is probably because of the difference of bond strength. The bond strength of Si-O is slightly higher than Si-N. ${ }^{38}$ To prevent oxygen atoms from forming bonds with silicon atoms on the surface of the quantum dots, chemical reactions were conducted on the silicon quantum dots capped with 1,5-hexadiene to make functionalised silicon quantum dots as is described in next chapter.

From ${ }^{1} \mathrm{H}$ NMR analysis of experiment 6, the signals from double bond were observed after the reaction with silicon quantum dots, and the sample was dried under vacuum at $60{ }^{\circ} \mathrm{C}$. Since most of the capping agents studied here have low boiling points, after the product was dried under vacuum, all the free capping molecules should also be removed. Therefore the result indicates that diene molecules did not bridge over two quantum dots. It is probably because of steric effects. Since the platinum catalyst has to bind to the terminal double bonds of the surface molecules of the silicon quantum dots if the carbon chain is shorter it casus more steric hindrance. The success of this surface modification brought a motivation to conduct some chemical reactions on the double bonds remaining on the surface of the silicon quantum dots. 
Table 3-1-1: Summary table of the reactions conducted in chapter 3

\begin{tabular}{|c|c|c|c|}
\hline Exp. & Capping agent & Capping method & $\begin{array}{c}\text { Particle size } \\
\text { (nm) }\end{array}$ \\
\hline 1 & Allylalcohol & Pt catalyst & no particles \\
\hline 2 & 1-hexene & Pt catalyst & $4.7 \pm 0.9$ \\
\hline 3 & 1,9 -decadiene & Pt catalyst & cannot define \\
\hline 4 & 1,5 -hexadienee & Pt catalyst & $4.8 \pm 2.7$ \\
\cline { 3 - 4 } & & UV-irradiation & $2.5 \pm 1.6$ \\
\hline 5 & allylamine & Pt catalyst & no particles \\
\cline { 3 - 4 } & & UV-irradiation & $4.1 \pm 1.6$ \\
\hline 6 & limonene & UV-irradiation & $4.7 \pm 0.5$ \\
\hline 7 & propagylamine & UV-irradiation & cannot define \\
\hline
\end{tabular}




\section{3-4. References}

1. D. C. Lee, T. Hanrath, B. A. Korgel; Angew. Chem Int Ed., 2005, 44, 35733577, "The Role of Precursor-Decomposition Kinetics in Silicon-Nanowire Synthesis in Organic Solvents”

2. L. E. Pell, A. D. Schricker, F. V. Mikulec, B. A. Korgel; Langmuir, 2004, 20, 6546-6548, "Synthesis of Amorphous Silicon Colloids by Trisilane Thermolysis in High Temperature Supercritical Solvents”

3. D. S. English, L. E. Pell, Z. H. Yu, P. F. Barbara, B. A. Korgel; Nano Lett., 2002, 2, 681-685, "Size Tunable Visible Luminescence from Individual Organic Monolayer Stabilized Silicon Nanocrystal Quantum Dots”

4. J. D. Holmes, K. J. Ziegler, R. C. Doty, L. E. Pell, K. P. Johnston, B. A. Korgel; J. Am. Chem. Soc., 2001, 123, 3743-3748, "Highly Luminescent Silicon Nanocrystals with Discrete Optical Transitions”

5. D. Zhifeng, M. Q. Bernadette, K. H. Santosh, L. E. Pell., B. A. Korgel., and J. B. Allen; Science, 2002, 296, 1293-1297, “Electrochemistry and Electrogenerated Chemiluminescence from Silicon Nanocrystal Quantum Dots”

6. A. S. Heintz, M. J. Fink, and B. S. Mitchell; Avd. Mater., 2007, 19, 3984-3988, "Mechanochemical Synthesis of Blue Luminescent Alkyl/Alkenyl-Passivated Silicon Nanoparticles”

7. A. S. Heintz, M. J. Fink, and B. S. Mitchell; Appl. Organometal. Chem., 2010, 24, 236-240, "Silicon nanoparticles with chemically tailored surfaces"

8. R. D. Tilley, J. H. Warner, K. Yamamoto, I. Matsui, and H. Fujimori; Chem. Commun., 2005, 14, 1833-1835, "Micro-emulsion synthesis of mono disperse surface stabilized silicon nanocrystals” 
9. J. H. Warner, A. Hoshino, K. Yamamoto, R. D. Tilley; Angew, 2005, 44, 4550-4554, "Water-Soluble Photoluminescent Silicon Quantum Dots”

10. A. Shiohara, S. Hanada, S. Prabakar, K. Fujioka, T. H. Lim, K. Yamamoto, P. T. Northcote, and R. D. Tilley; J. Am. Chem. Soc., 2010, 132, 248-253, “Chemical Reactions on Surface Molecules Attached to Silicon Quantum Dots”

11. D. Neiner, H. W. Chiu, S. M. Kauzlarich; J. Am. Chem. Soc., 2006, 128, 11016-11017, “Low-Temperature Solution Route to Macroscopic Amounts of Hydrogen Terminated Silicon Nanoparticles”

12. J. Zou, R. K. Baldwin, K. A. Pettigrew, S. M. Kauzlarich; Nano Lett., 2004, 4, 1181-1186, “Solution Synthesis of Ultrastable Luminescent Siloxane-Coated Silicon Nanoparticles”

13. Q. Liu, S. M. Kauzlarich; Mater. Sci. Eng. B, 2002, 96, 72-75, “A new synthetic route for the synthesis of hydrogen terminated silicon nanoparticles”

14. D. Neiner, and S. M. Kauzlarich; Chem. Mater., 2010, 22, 487-493, “Hydrogen-Capped Silicon Nanoparticles as a Potential Hydrogen Storage Material; Synthesis, Characterization, and Hydrogen Release”

15. R. K. Baldwin, K. A. Pettigrew, E. Ratai, M. P. Augustine, S. M. Kauzlarich; Chem. Comm., 2002, 1822-1823, "Solution reduction synthesis of surface stabilized silicon nanoparticles”

16. R. K. Baldwin, K. A. Pettigrew, J. C. Garno, P. P. Power, G. Y. Liu, S. M. Kauzlarich; J. Am. Chem. Soc., 2002, 124, 1150-1151, "Room Temperature Solution Synthesis of Alkyl-Capped Tetrahedral Shaped Silicon Nanocrystals” 
17. D. Mayeri, B. L. Phillips, M. P. Augustine, S. M. Kauzlarich; Chem. Mater., 2001, 13, 765-770, "NMR Study of the Synthesis of Alkyl-Terminated Silicon Nanoparticles from the Reaction of $\mathrm{SiCl}_{4}$ with the Zintl Salt, NaSi”

18. C. S. Yang, R. A. Bley, S. M. Kauzlarich, H. W. H. Lee, G. R. Delgado; J. Am. Chem. Soc., 1999, 121, 5191-5195, "Synthesis of Alkyl-Terminated Silicon Nanoclusters by a Solution Route"

19. R. A. Bley, S. M. Kauzlarich; J. Am. Chem. Soc., 1996, 118, 12461-12462, “A Low-Temperature Solution Phase Route for the Synthesis of Silicon Nanoclusters”

20. S. H. Hwang, C. N. Moorefield, P. Wang, K. U. Jeong, S. D. Z. Cheng, K. K. Kotta, and G. R. Newkome; Chem. Commun., 2006, 33, 3495-3497, "Construction of CdS quantum dots via a regioselective dendritic functionalized cellulose template”

21. E. R. Goldman, E. D. Balighian, H. Mattoussi, M. K. Kuno, J. M. Mauro, P. T. Tran, G. P. Anderson; J. Am. Chem. Soc., 2002, 124, 6378-6382, “Avidin: A Natural Bridge for Quantum Dot-Antibody Conjugates”

22. E. R. Goldman, G. P. Anderson, P. T. Tran, H. Mattoussi, P. T. Charles, J. M. Mauro; Anal. Chem., 2002, 74, 841-847, “Conjugation of Luminescent Quantum Dots with Antibodies Using an Engineered Adaptor Protein To Provide New Reagents for Fluoroimmunoassays”

23. J. P. Wilcoxon, G. A. Samara, P. N. Provencio; Phys. Rev. B, 1999, 60, 27042714, "Optical and electronic properties of Si nanoclusters synthesized in inverse micelles”

24. J. P. Wilcoxon, G. A. Samara; Appl. Phys. Lett., 1999, 74, 3164-3166, “Tailorable, visible light emission from silicon nanocrystals”

25. Z. F. Li, E. Ruckenstein; Nano Lett., 2004, 4, 1463-1467, "Water-Soluble 
Poly(acrylic acid) Grafted Luminescent Silicon Nanoparticles and Their Use as Fluorescent Biological Staining Labels”

26. F. Hua, F. Erogbogbo, M. T. Swihart, and E. Ruckenstein; Langmuir, 2006, 22, 4363-4370, “Organically Capped Silicon Nanoparticles with Blue Photoluminescence Prepared by Hydrosilylation Followed by Oxidation”

27. F. Hua, M. T. Swihart, and E. Ruckenstein; Langmuir, 2005, 21, 6054-6062, "Efficient surface grafting of luminescent silicon quantum dots by photoinitiated hydrosilylation”

28. S. Sato, M. T. Swihart; Chem. Mater., 2006, 18, 4083-4088, "Propionic-AcidTerminated Silicon Nanoparticles: Synthesis and Optical Characterization”

29. A. Gupta, M. T. Swihart, and H. Wiggers; Adv. Funct. Mater., 2009, 19, 696703, "Luminescent Colloidal Dispersion of Silicon Quantum Dots from Microwave Plasma Synthesis: Exploring the Photoluminescence Behavior Across the Visible Spectrum"

30. F. Erogbogbo, K. T. Yong, I. Roy, G. Xu, P. N. Prasad, and M. T. Swihart; ACS Nano, 2008, 2, 873-878, “Bioconpatible Luminescent Silicon Quantum Dots for Imaging of Cancer Cells"

31. R. M. Silverstein, and F. X. Webster; Spectrometric Identification of Organic Compounds, John Wiley \& Sons, Inc., New York, United States of America, 1996.

32. http://oldwebsite.laurentian.ca/chem/ssiemann/teaching/3317/metallic.pdf

33. http://booklists.narod.ru/Ch_Chemistry/Chambers_Holliday._Modern_inorga nic_chemistry_1975__T_459s_.5.htm 
34. P. L. Crowell, S. Lin, E. Vedejs, and M. N. Gould; Cancer Chemother Pharmacol., 1992, 31, 205-212, "Identification of metabolites of the antitumor agent d-limonene capable of inhibiting protein isoprenylation and cell growth”

35. J. E. Martin, J. P. Wilcoxon, J. Odinek, and P. Provencio; J. Phys. Chem. B 2000, 104, 9475-9486, "Control of the Interparticle Spacing in Gold Nanoparticle Superlattices”

36. W. W. Yu, E. Chang, J. C. Falkner, J. Zhang, A. M. Al-Somali, C. M. Sayes, J. Johns, R. Drezek, and V. L. Colvin; J. Am. Chem. Soc., 2007, 129, 2871-2879, "Forming Biocompatible and Nonaggregated Nanocrystals inWater Using Amphiphilic Polymers”

37. Y. Vahidshad, H. Abdizadeh, M. Akbari, and H. R. Baharvandi; J. Sol-Gel Sci. Technol., 2010, 53, 263-271, "Size-controlled synthesis of $\mathrm{CuO}-\mathrm{ZrO}_{2}$ nanoparticles prepared through reverse micelle method”

38. http://www.wiredchemist.com/chemistry/data/bond_energies_lengths.html 


\section{Chapter 4 Chemical reactions on the surface of silicon quantum dots and their biological application}

This chapter describes further chemical reactions on the surface of silicon quantum dots towards biological applications. Single step surface modification of silicon quantum dots with terminal alkenes were described in chapter 3. However during the trials we identified problems with the functional groups such as the hydroxyl group or carboxyl group. Since silicon-oxygen bond $(531.37 \mathrm{~kJ} / \mathrm{mol})$ is more stable than silicon-carbon bond(317.98 $\mathrm{kJ} / \mathrm{mol}){ }^{1}$, the oxygen atom from these functional groups tends to attach to silicon atoms on the surface of quantum dots and destroyed the functionalities which were supposed to remain on the terminal of the quantum dots. The preparation of water-dispersible silicon quantum dots that maintain their photoluminescence stability has been proven difficult.

There have been several reports published on surface modification of silicon quantum dots. As described in the introduction chapter, silicon quantum dots have been reported coated with poly acrylic acid (PAAc) by Ruckenstein et al. ${ }^{2}$ Silicon quantum dots have also been achieved coated with propionic acid by Swihart et al. In both cases, silicon quantum dots were capped under $254 \mathrm{~nm}$ of UV light irradiation. ${ }^{3}$ However both of the reports are focused on the optical properties rather than in the characterisation of the surface molecules. The surface molecules of those silicon quantum dots have been characterised only by FTIR analysis by Swihart. ${ }^{3}$ Kauzlarich et. al reported paramagnetic manganese-doped silicon quantum dots coated with dextran sulphate. ${ }^{4}$ They used allylamine capped silicon quantum dots to be conjugated with dextran, however in these studies the surface molecules were also characterised only by FTIR analysis. To the best of our knowledge, there are very few reports describing chemical reactions on the surface molecules of silicon quantum dots. Even these reports hardly showed full characterisation of the surface molecules. 
In this research we describe the chemical reactions on the surface molecules of the silicon quantum dots and show the full characterisation of surface molecules of the silicon quantum dots. The purpose of the chemical reactions described in this chapter is the functionalisation of the silicon quantum dots by breaking the carbon carbon double bond of the attached diene. This is important because it will allow a larger range of functionalities to be utilised which would usually attach directly to the silicon quantum dots via their oxygen functionality or ineffectively protect the silicon surface.

We applied NMR and FTIR analysis in order to characterise the surface molecules of the silicon quantum dots. In particular, NMR technique can give much more specific information on the surface molecules of the silicon quantum dots than FTIR analysis however, not many NMR data of the silicon quantum dots have been reported.

Photoluminescence measurements were carried out for the silicon quantum dots. It turned out that the type of capping agents attached on the surface of the silicon quantum dots have effects on the optical properties of the quantum dots. The optical properties of the silicon quantum dots with different surface molecules and the difference in the optical properties depending on the surface molecules will be discussed.

Also cell viability of the silicon quantum dots after functionalized was assessed by MTT assay ${ }^{5,6}$ to determine the threshold (50 \% inhibition coefficients) concentration of the silicon quantum dots with different capping molecules on several different cell types. Different surface modifications can induce different levels of quantum dot toxicity. Thus, a detailed examination of the toxicity of the quantum dots with various capping molecules is essential for the widespread understanding of the application of quantum dots in biological imaging.

After the assessment of cell viability of silicon quantum dots, amine terminated quantum dots were used for bio-imaging to ascertain the cell uptake of silicon quantum dots. 


\section{4-1. Chemical reactions of the surface moieties of the silicon quantum dots}

\section{Experiment 4-1-1: Epoxidation of terminal double bond of silicon quantum dots}

Epoxide terminated silicon quantum dots were synthesised from the silicon quantum dots capped with 1,5-hexadiene from Experiment 6 from chapter 3. Epoxide is a highly reactive group and is easily oxidised to form a hydroxyl group. Therefore it is very difficult to apply molecules which have epoxy groups as capping agent since it has high potential to react with silicon atoms on the surface of the quantum dots. Therefore we applied the strategy of multiple steps to produce epoxide terminated silicon quantum dots by reacting the carbon-carbon double bond at the terminal end of the surface of the silicon quantum dots. The terminal double bond was oxidised using m-chloroperoxy benzoic acid (m-CPBA) in dichloromethane. The surface molecules were characterised by FTIR and ${ }^{1} \mathrm{H}$ NMR analysis. The reaction on the free molecules was conducted with 1-hexene and m-CPBA.
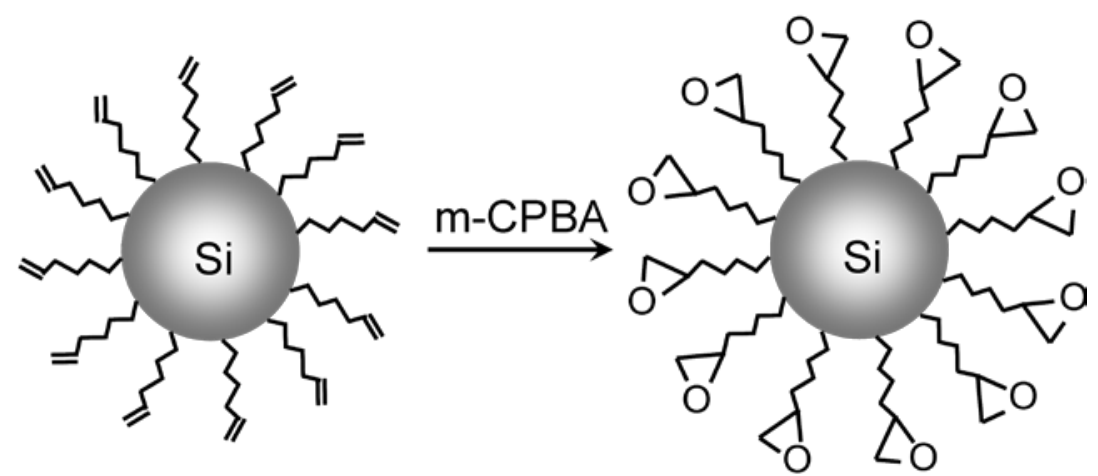

Reaction scheme for the termination of silicon quantum dot 


\section{${ }^{1} \mathrm{H}$ NMR result of the reaction on the free molecules}

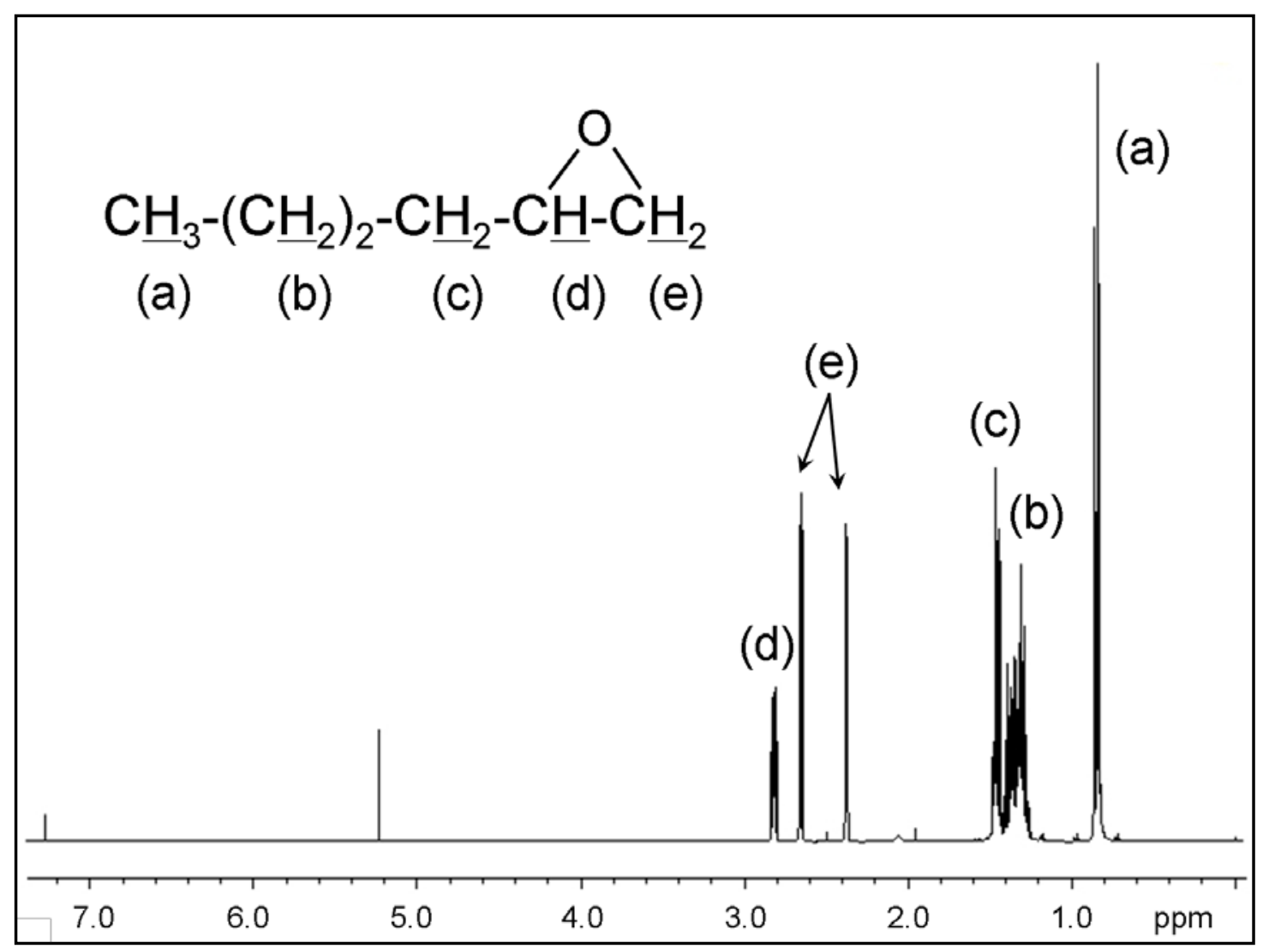

Figure 4-1: ${ }^{1} \mathrm{H}$ NMR spectrum of the product obtained from the reaction on the free molecules

${ }^{1} \mathrm{H}$ NMR (500MHz, $\left.\mathrm{CDCl}_{3}\right)$

$\delta: 0.84$ ppm (3H, t, H-(a), J=7.3 Hz), $\delta: 1.30$ ppm (4H, m, H-(b)), $\delta: 1.46$ ppm (2H, m, H-(c)), $\delta: 2.38$ ppm (1H, dd, H-(e) J=2.6, $5.1 \mathrm{~Hz}$ ), $\delta: 2.65$ ppm (1H, dd, H-(e), $J=3.9,5.1 \mathrm{~Hz}), \delta: 2.82 \mathrm{ppm}(1 \mathrm{H}, \mathrm{m}, \mathrm{H}-(\mathrm{d}))$

After treating 1-hexene with $\mathrm{m}-\mathrm{CPBA}$, the ${ }^{1} \mathrm{H}$ NMR showed that the signals indicative of a double bond around 5-6 ppm had disappeared. Three sharp peaks were observed at $2.38 \mathrm{ppm}, 2.65 \mathrm{ppm}$ and $2.82 \mathrm{ppm}$. Each signal integrates for one proton which corresponds to H-(d) and H-(e) of the epoxy ring. The triplet signal observed at 0.84 ppm integrates for the three protons of the methyl group, H-(a). Signals of H-(b) and $\mathrm{H}$-(c) were observed as multiplets at $1.30 \mathrm{ppm}$ and $1.46 \mathrm{ppm}$ respectively. 


\section{Results of the reaction on the silicon quantum dots}

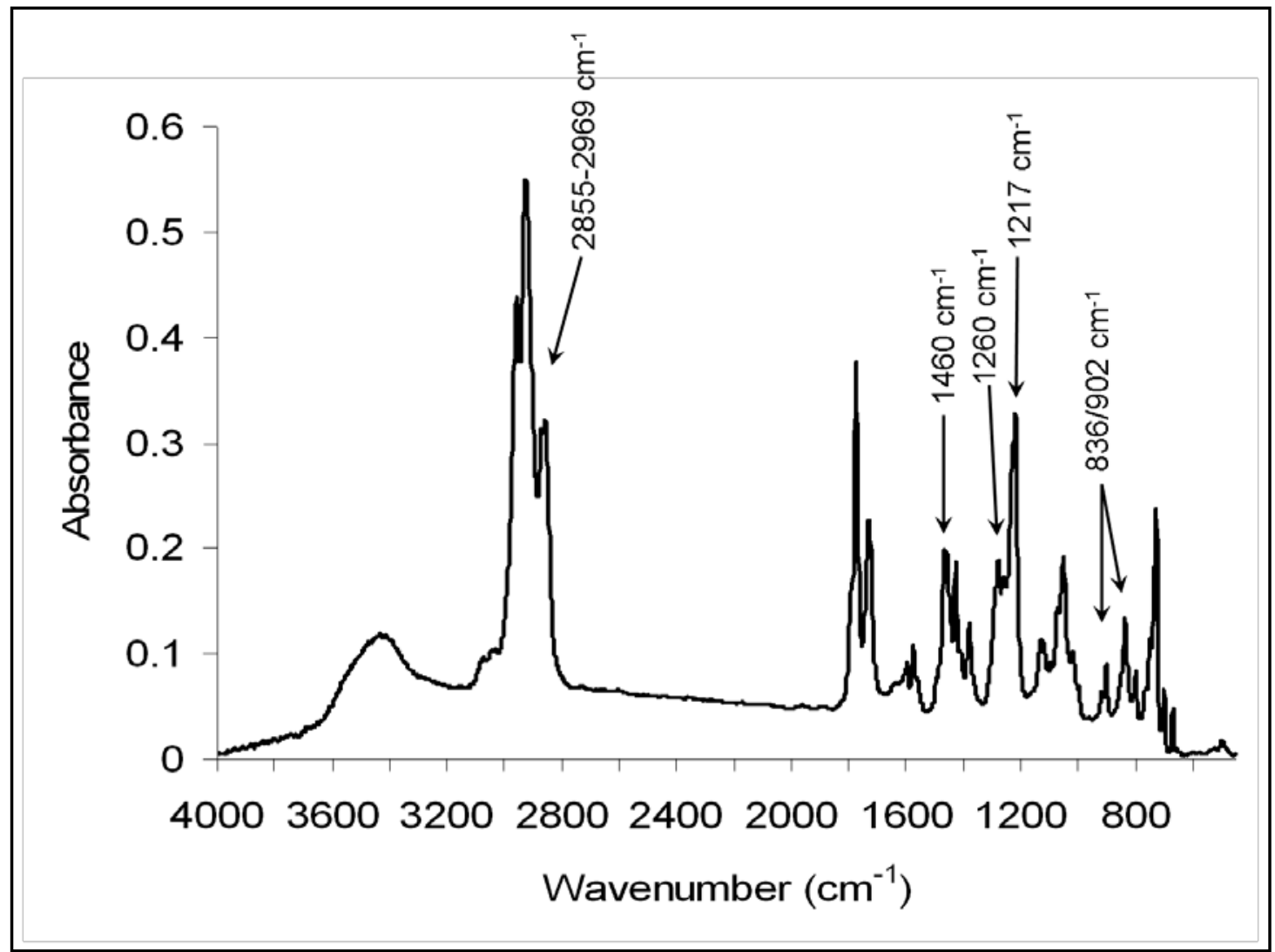

Figure 4-2: FTIR spectrum of the sample obtained from experiment 4-1-1

As may be seen in the FITR spectrum (Figure 4-2), epoxy ring symmetrical and asymmetrical stretching was observed at $1217 \mathrm{~cm}^{-1}$ and 836-902 $\mathrm{cm}^{-1}$. ${ }^{7}$ Also Si-CH vibrational scissoring can be seen at $1260 \mathrm{~cm}^{-1}$ and $1460 \mathrm{~cm}^{-1}$. The peak corresponds to $\mathrm{C}=\mathrm{C}$ stretching at $1640 \mathrm{~cm}^{-1}$ could not be seen in this spectrum. The peaks at 2855$2969 \mathrm{~cm}^{-1}$ represent alkyl chain of the surface moieties of the silicon quantum dots. The peak observed at $1773 \mathrm{~cm}^{-1}$ possibly represent the carbonyl $\mathrm{C}=\mathrm{O}$ stretching from the oxidising agent, m-CPBA. ${ }^{7}$

NMR spectrum also supported the presence of an epoxy ring. Two singlets observed at $2.39 \mathrm{ppm}$ and $2.67 \mathrm{ppm}$ represent protons attached to the terminal carbon (H-(e)). The epoxy ring restricts the rotation of the carbon-carbon bond of the ring, and thus made the different chemical environment for these two protons. H-(d) is less shielded compared to the H-(e) protons therefore the peak of $\mathrm{H}$-(d) is observed at $2.84 \mathrm{ppm}$ as a singlet. The signals at $0.81 \mathrm{ppm}, 1.18 \mathrm{ppm}$ and $1.44 \mathrm{ppm}$ possibly represent H-(a), 
$\mathrm{H}$-(b) and $\mathrm{H}$-(c) respectively, however these are probably overlapped with the surfactant signals as TOAB also has signals around the same region.

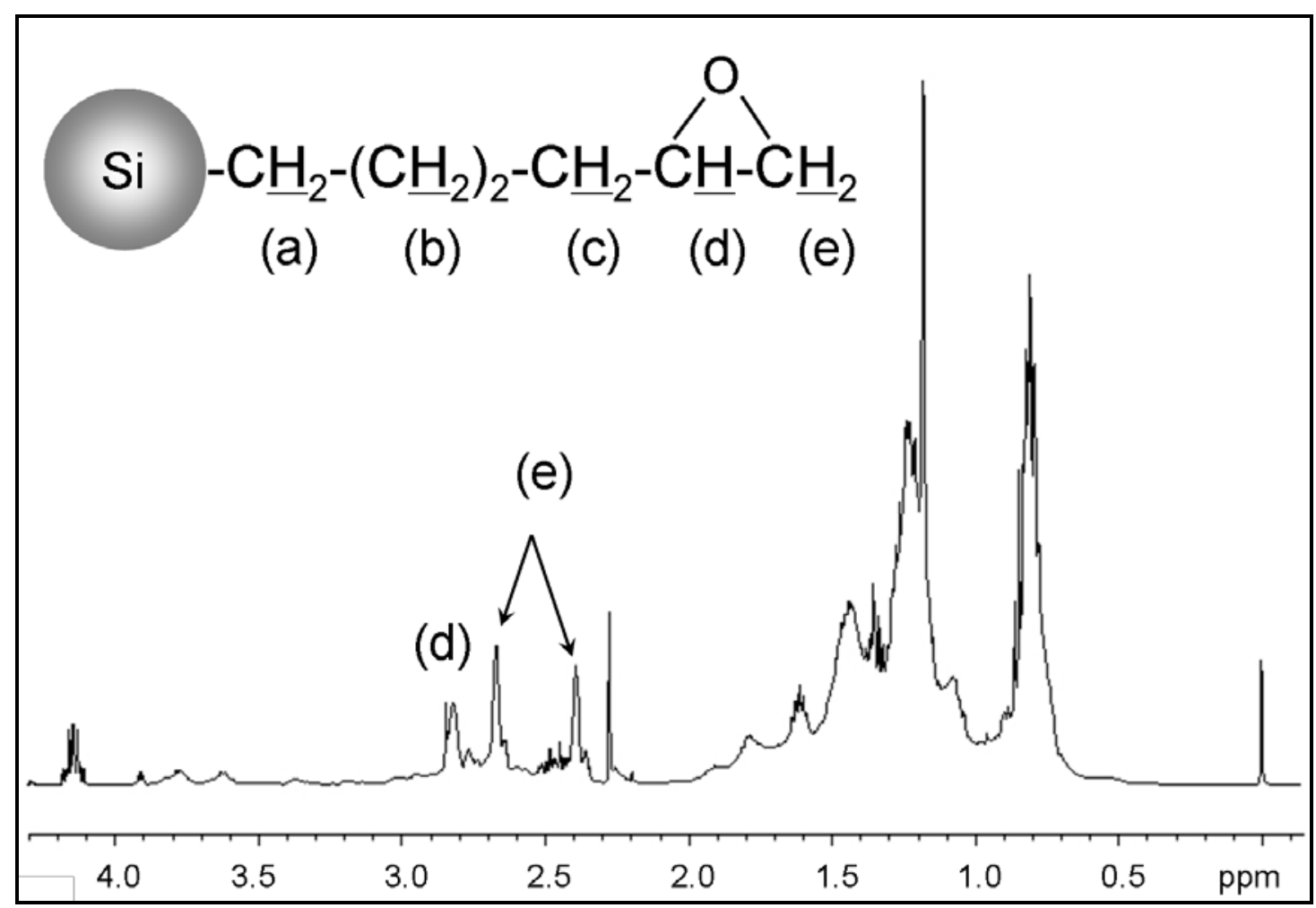

Figure 4-3: ${ }^{1} \mathrm{H}$ NMR spectrum of silicon quantum dots with epoxide termination obtained from experiment 4-1-1

${ }^{1} \mathrm{H}$ NMR (500MHz, $\mathrm{CDCl}_{3}$ )

$\delta: 2.39$ ppm (1H, s, H-(e)), $\delta: 2.67$ ppm (1H, s, H-(e)), $\delta: 2.84$ ppm (1H, d, H-(d) $J=12.7 \mathrm{~Hz})$

\section{Experimental}

Silicon quantum dots capped with 1,5-hexadiene from experiment 6 from chapter 3 were dissolved in $10 \mathrm{~mL}$ of dichloromethane. $0.30 \mathrm{~g}$ (1.5 eq) of m-CPBA was added and the reaction mixture was stirred at room temperature for 6 hours. A saturated solution of sodium sulfite was added to quench m-CPBA. ${ }^{9}$ The aqueous phase was extracted with dichloromethane for three times and the combined organic layers were washed with a $10 \%$ sodium hydroxide solution, dried over magnesium sulphate, filtered and concentrated under reduced pressure. 


\section{Experiment 4-1-2: Oxidation of terminal epoxy ring of silicon quantum dots}

For the next step, silicon quantum dots with a diol group on the surface were synthesised from the silicon quantum dots with epoxy ring obtained from experiment 4-1-1. For the biological applications to make the silicon quantum dots water soluble is a crucial step. The terminal diol groups can make the silicon quantum dots water soluble and also broaden the possibilities of attaching bio-compatible molecules to give them greater functionality.

The terminal epoxy ring was oxidised by water using the phase transfer catalyst, tetrabutylammonium sulphate $\left(\mathrm{Bu}_{4} \mathrm{NHSO}_{4}\right)$ to form the diol group. ${ }^{10}$

A reaction on the free molecules was conducted using the epoxy hexane produced from the reaction on 1-hexene of experiment 4-1-1. Epoxy hexane in chloroform was treated with water in the presence of $\mathrm{Bu}_{4} \mathrm{NHSO}_{4}$. The product was characterised by ${ }^{1} \mathrm{H}$ NMR.

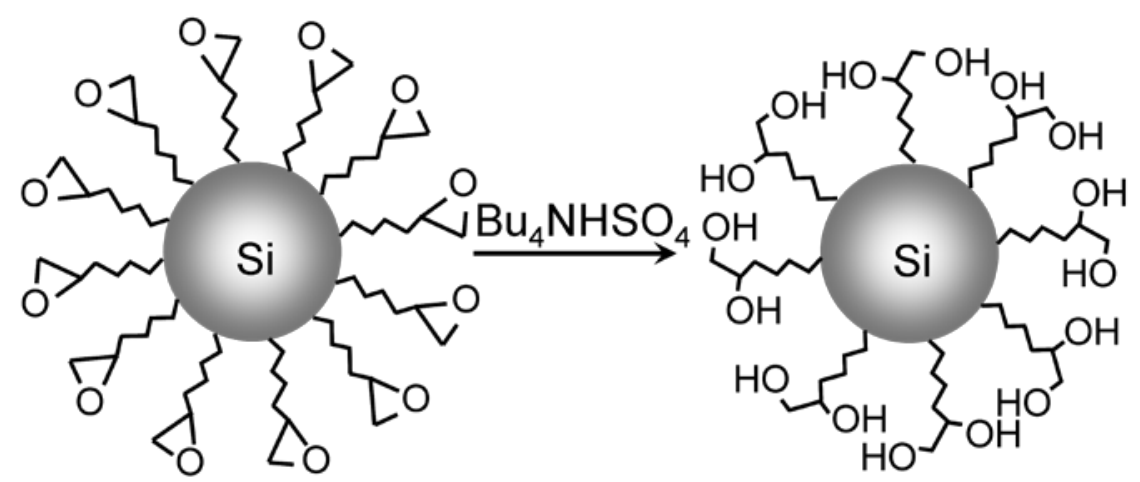

Reaction scheme of diol terminated silicon quantum dot 


\section{${ }^{1} \mathrm{H}$ NMR result of the reaction on the free molecules}

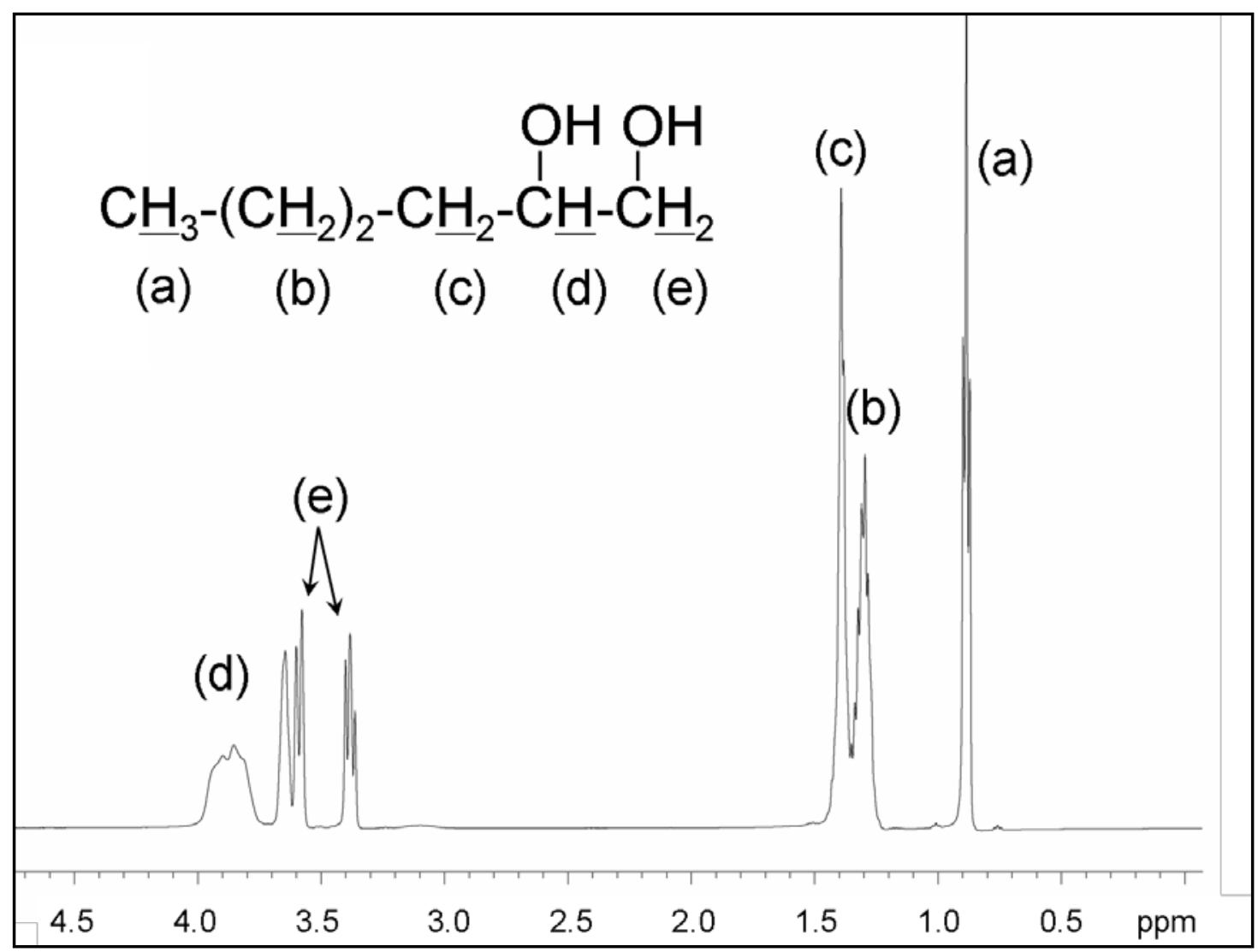

Figure 4-4: ${ }^{1} \mathrm{H}$ NMR spectrum of reaction on the free molecules for experiment 4-1-2

${ }^{1} \mathrm{H}$ NMR (500MHz, $\left.\mathrm{CDCl}_{3}\right)$

$\delta: 0.88$ ppm (3H, t, H-(a), J=6.6 Hz), $\delta: 1.30$ ppm (4H, m, H-(b)), $\delta: 1.39$ ppm (2H, t, H-(c) $J=5.2 \mathrm{~Hz}$ ), 3.38 ppm (1H, t, H-(e), J=9.1 Hz), 3.58 ppm (1H, t, H-(e), J=11.4 Hz), 3.85 ppm (1H, m, H-(d))

As can be seen in Figure 4-4, a triplet was observed at $0.88 \mathrm{ppm}$ which indicates a terminal methyl group. Four protons of $\mathrm{H}$-(b) can be seen at $1.30 \mathrm{ppm}$ as a multiplet. The sharp signal at $1.39 \mathrm{ppm}$ represents the two protons of $\mathrm{H}-(\mathrm{c})$. The two protons of $\mathrm{H}$-(e) are observed as doublets at $3.38 \mathrm{ppm}$ and $3.58 \mathrm{ppm}$. H-(d) was seen as a broad signal at $3.85 \mathrm{ppm}$. The each signal at $0.88 \mathrm{ppm}, 1.30 \mathrm{ppm}$ and $1.39 \mathrm{ppm}$ integrates for three protons, four protons and two protons, respectively, which correspond to the number of the protons of each group. 

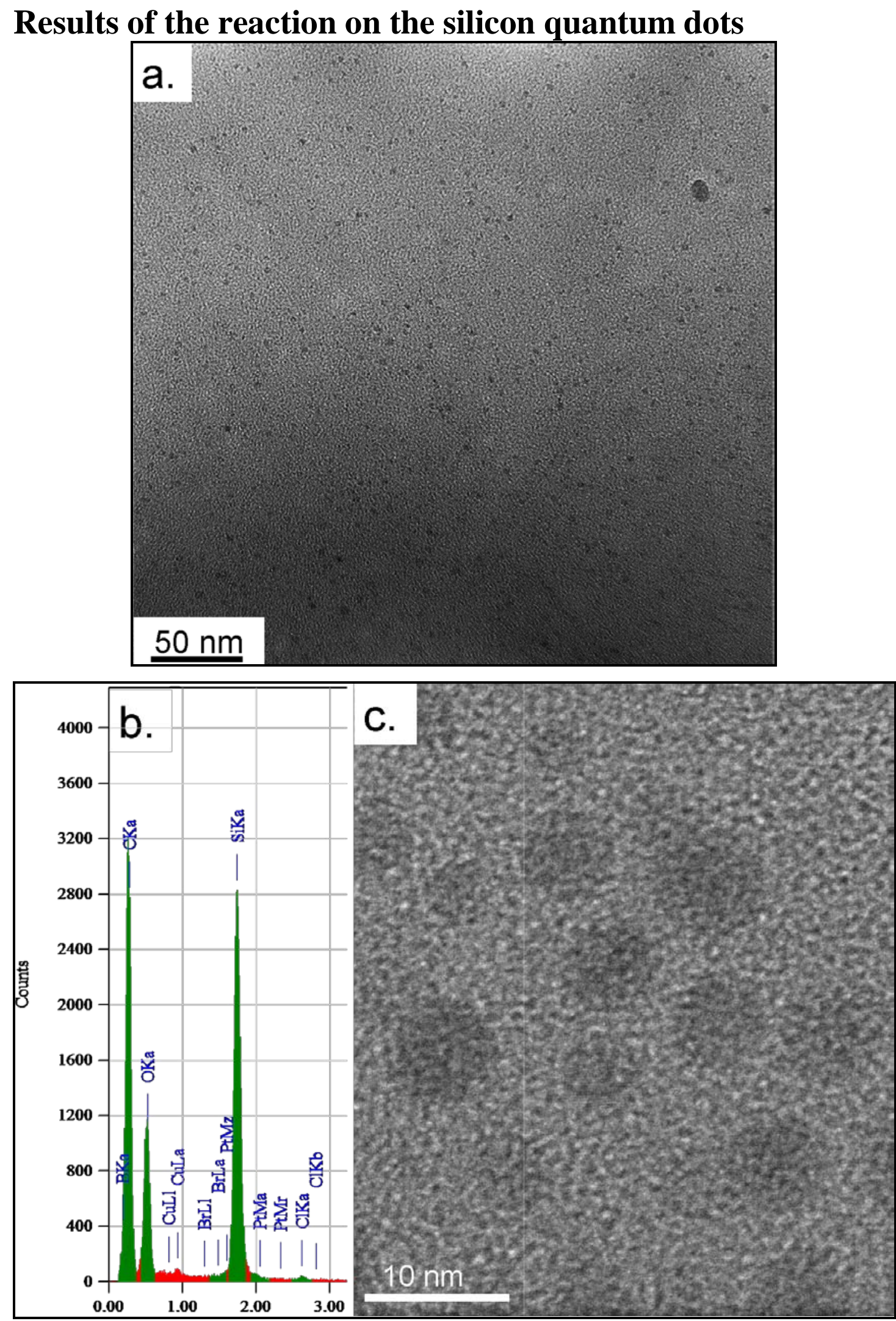

Figure 4-5: (a) a low resolution TEM micrograph of silicon quantum dots capped with 1,2-hexandiol; (b) EDS spectrum corresponding to the nanoparticles shown in (a); (c) a high resolution TEM micrograph of silicon quantum dots obtained from experiment 4-1-2, (d); a graph of size distribution 


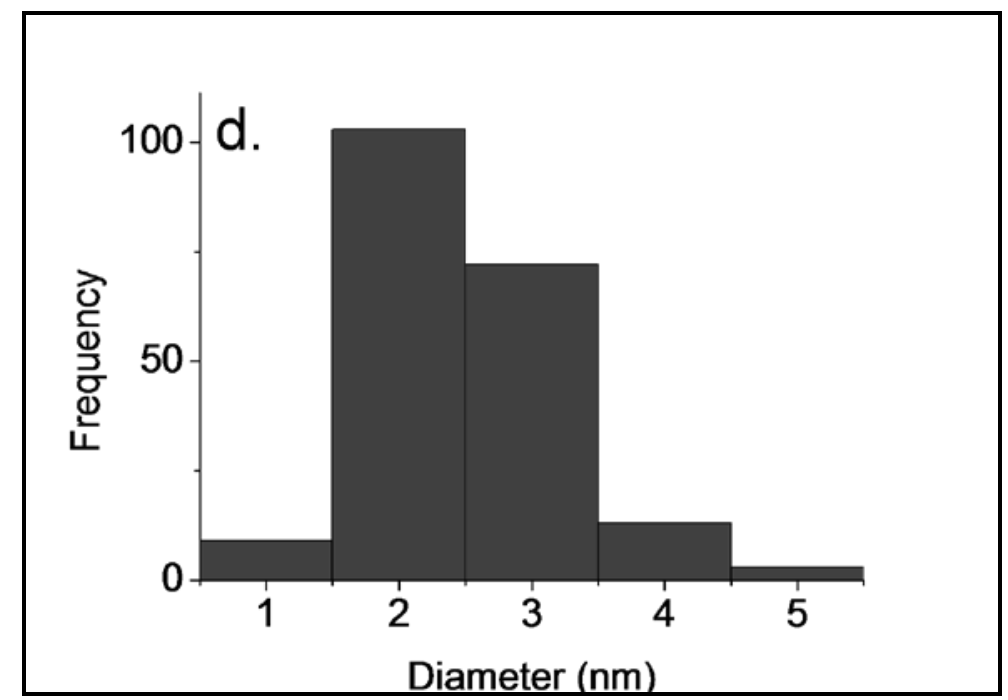

Figure 4-5: (a) a low resolution TEM micrograph of silicon quantum dots capped with 1,2-hexandiol; (b) EDS spectrum corresponding to the nanoparticles shown in (a); (c) a high resolution TEM micrograph of silicon quantum dots obtained from experiment 4-1-2, (d); a graph of size distribution

As may be seen in Figure 4-5, the diol-capped nanoparticles were observed by HRTEM micrograph. Majority of the nanoparticles have a spherical shape and an average size of $2.7 \pm 0.4 \mathrm{~nm}$ which is very close to the Bohr radius of silicon. However there are some bigger size nanoparticles around $10 \mathrm{~nm}$ were observed as well. EDS analysis showed the nanoparticles were composed of mainly silicon. Since silicon is a very light material compared to other heavy metals, SAED could not be obtained from this area. Lattice fringes of crystalline silicon could not be observed from the high resolution TEM micrograph either. However since the silicon quantum dots capped with 1,5-hexadiene obtained from experiment 6 from chapter 3 which was used for this experiment have been confirmed as crystalline silicon by SAED, these nanoparticles were believed to be crystalline silicon as well.

In the FTIR spectrum, a broad O-H stretching peak can be seen at $3410 \mathrm{~cm}^{-1}$ which indicates intermolecular hydrogen bonding. Also $\mathrm{O}-\mathrm{H}$ in plane bending is observed at $1464 \mathrm{~cm}^{-1}$ and $1380 \mathrm{~cm}^{-1}$. The peaks at $1072 \mathrm{~cm}^{-1}, 1123 \mathrm{~cm}^{-1}$ and $1260 \mathrm{~cm}^{-1}$ correspond to $\mathrm{C}-\mathrm{O}$ stretching at the terminal $\mathrm{OH}$ groups. $\mathrm{Si}-\mathrm{CH}_{2}$ vibrational scissoring can also be seen at $1260 \mathrm{~cm}^{-1}$ and $1460 \mathrm{~cm}^{-1}$ which probably overlapped with the $\mathrm{O}-\mathrm{H}$ in plane bending and C-O stretching. The peaks from $2850 \mathrm{~cm}^{-1}$ to $2956 \mathrm{~cm}^{-1}$ represent $\mathrm{C}-\mathrm{CH}_{2}$ symmetrical, asymmetrical stretching. The peak corresponding to $\mathrm{C}=\mathrm{C}$ stretching at $1640 \mathrm{~cm}^{-1}$ and the peak at $1217 \mathrm{~cm}^{-1}$ corresponding to the epoxy 
ring which were seen in the FTIR spectra from experiments 4-1-1 could not be seen in this spectrum. This indicates the success of oxidation of the epoxy ring to form a diol group.

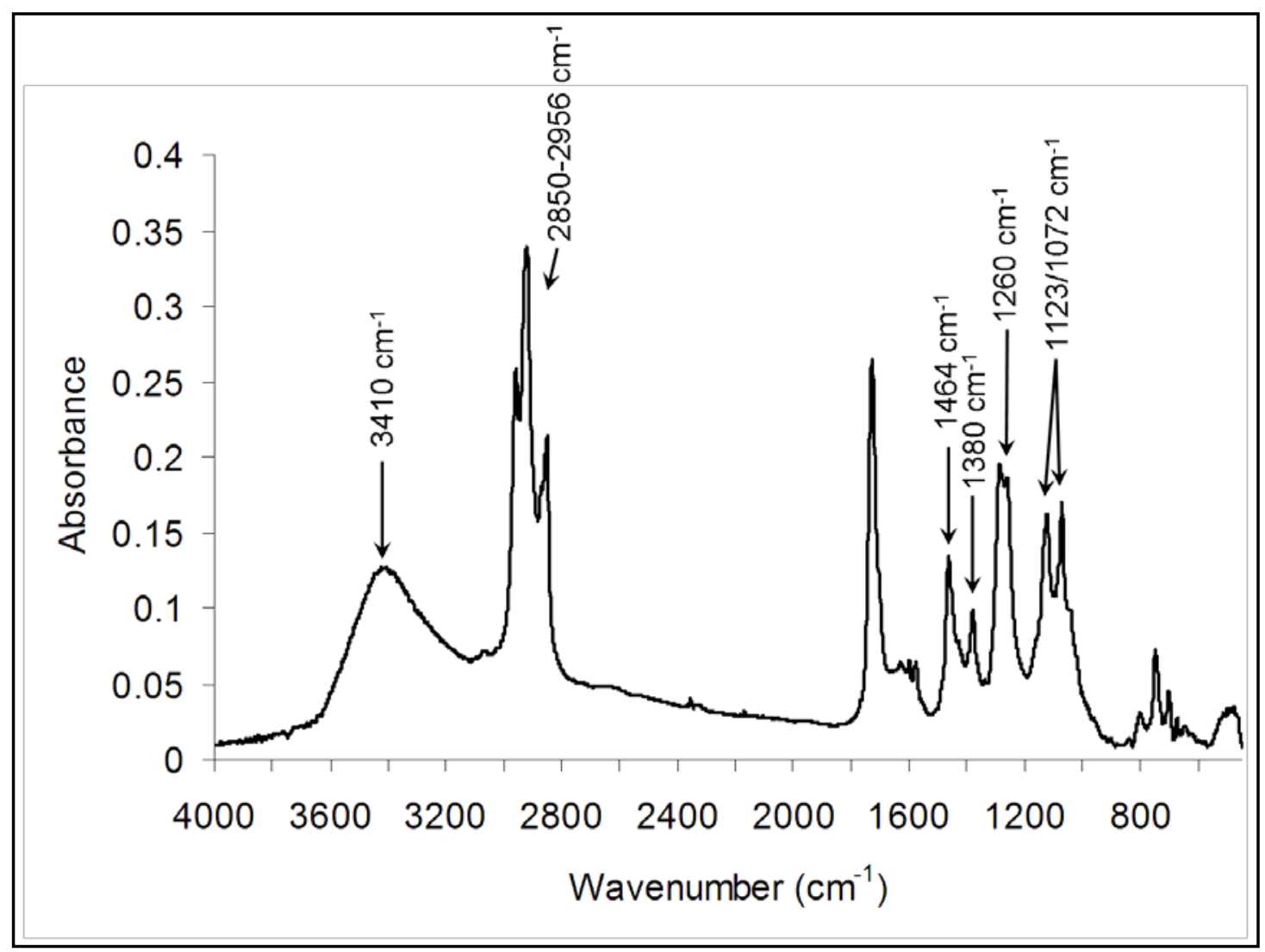

Figure 4-6: FTIR spectrum of silicon quantum dots with terminal diol group 


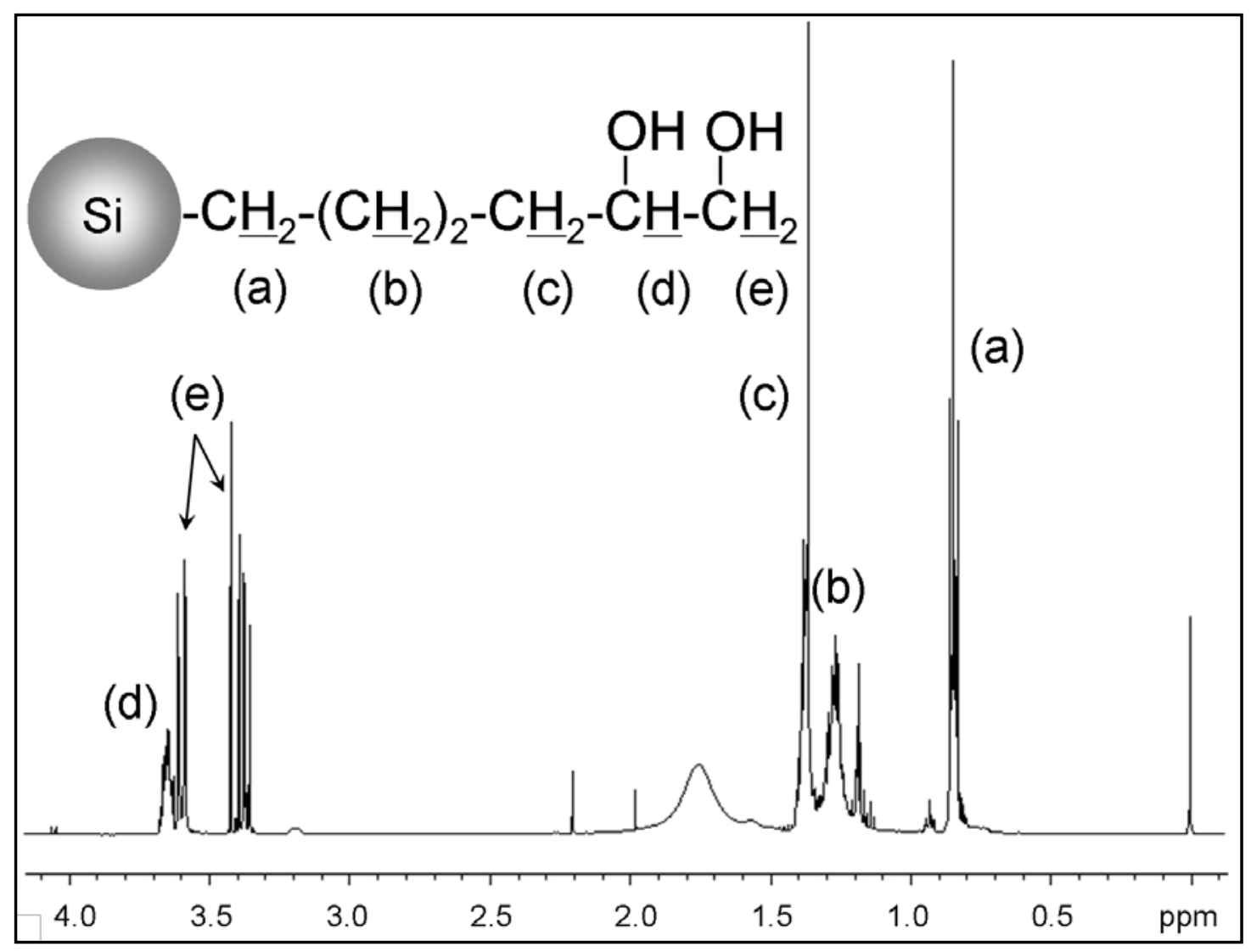

Figure 4-7: ${ }^{1} \mathrm{H}$ NMR spectrum of silicon quantum dots with terminal diol group

${ }^{1} \mathrm{H}$ NMR (500MHz, $\left.\mathrm{CDCl}_{3}\right)$

$\delta: 0.85 \mathrm{ppm}(2 \mathrm{H}, \mathrm{t}, \mathrm{H}-(\mathrm{a}), J=6.8 \mathrm{~Hz}), \delta: 1.27 \mathrm{ppm}(4 \mathrm{H}, \mathrm{m}, \mathrm{H}-(\mathrm{b})), \delta: 1.36 \mathrm{ppm}(2 \mathrm{H}$, m, H-(c)), $\delta: 3.39$ ppm (1H, dd, H-(e), J=7.6, 11.0 Hz), $\delta: 3.58$ ppm (1H, dd, H-(e), $J=2.9,10.9 \mathrm{~Hz}), \delta: 3.65 \mathrm{ppm}(1 \mathrm{H}, \mathrm{m}, \mathrm{H}-(\mathrm{d}))$

In ${ }^{1} \mathrm{H}$ NMR spectrum, overlapped two doublets could be seen at $3.39 \mathrm{ppm}$ which represent one of the $\mathrm{H}-(\mathrm{e})$. The other $\mathrm{H}-(\mathrm{e})$ signal came up also as two doublets at $3.58 \mathrm{ppm}$. Usually a carbon-carbon single bond has free rotation, however because of the two hydroxyl groups the structure of the surface moieties are probably sterically hindered therefore each proton of H-(e) has different chemical environments. This caused the different chemical shift for each proton of $\mathrm{H}-(\mathrm{e})$. H-(d) signal is observed at $3.65 \mathrm{ppm}$ as multiplets since this proton is less shielded compared to other protons. The triplet seen at $0.85 \mathrm{ppm}$ integrates for 2 protons. It possibly represents $\mathrm{H}$-(a). H(b) and $\mathrm{H}-(\mathrm{c})$ came up as multiplets at $1.27 \mathrm{ppm}$ and $1.36 \mathrm{ppm}$. The signals from the epoxy ring, $2.39 \mathrm{ppm}, 2.67 \mathrm{ppm}$ and $2.82 \mathrm{ppm}$ which correspond to the epoxy ring are not observed in this spectrum. The result from ${ }^{1} \mathrm{H}$ NMR spectrum indicated the 
success of oxidation of epoxy ring of the surface moieties of silicon quantum dots from experiment 4-1-1 to form diol.

To apply to biological conditions, the solubility in water of silicon quantum dots is a critical point. Therefore, the successful synthesis of silicon quantum dots with terminal diol groups has opened up the door for biological applications of these materials.

\section{Experimental}

Silicon quantum dots capped with epoxyhexane from experiment 4-1-1 was dissolved in $1 \mathrm{~mL}$ of chloroform and added to $2 \mathrm{~mL}$ of water. $2.0 \mathrm{mg}$ of $\mathrm{Bu}_{4} \mathrm{NHSO}_{4}$ was added to the solution mixture and stirred for 16 hours at $40{ }^{\circ} \mathrm{C}$. The mixture was extracted with $10 \mathrm{~mL}$ of diethyl ether for 3 times. The organic layers were combined and dried over $\mathrm{Na}_{2} \mathrm{SO}_{4}$. The solvent was removed under vacuum and the crude product was purified by silica gel column chromatography.

\section{Experiment 4-1-3: Bromination of terminal double bond of silicon quantum dots}

Silicon quantum dot capped with 1,2-dibromohexane was synthesised from silicon quantum dot capped with 1,5-hexadiene obtained from experiment 6 from chapter 3 . The aim of this experiment was to calculate the yield of the silicon quantum dots. The terminal double bonds of the silicon quantum dots capped with 1,5-hexadiene were brominated by titration with the known concentration of bromine solution. From the amount of bromine consumed in the titration and the estimated molecular weight of silicon quantum dot, the yield of the silicon quantum dots can be calculated. The reaction on the free molecules was conducted by titration of 1-hexene in dichloromethane with bromine solution and characterised by ${ }^{1} \mathrm{H}$ NMR. 


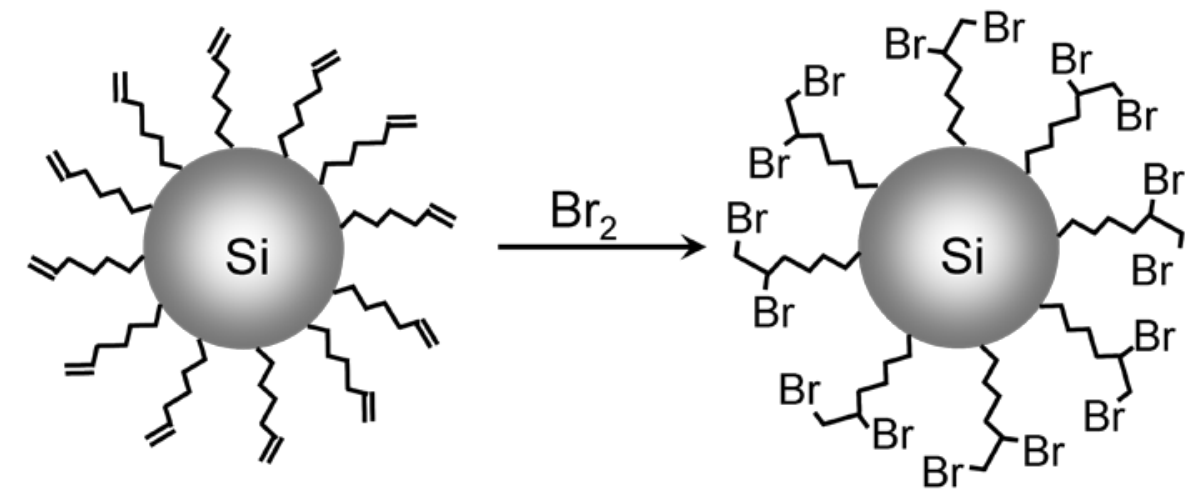

Reaction scheme of silicon quantum dot capped with 1,2-dibromohexane

${ }^{1} \mathrm{H}$ NMR result of the reaction on the free molecules

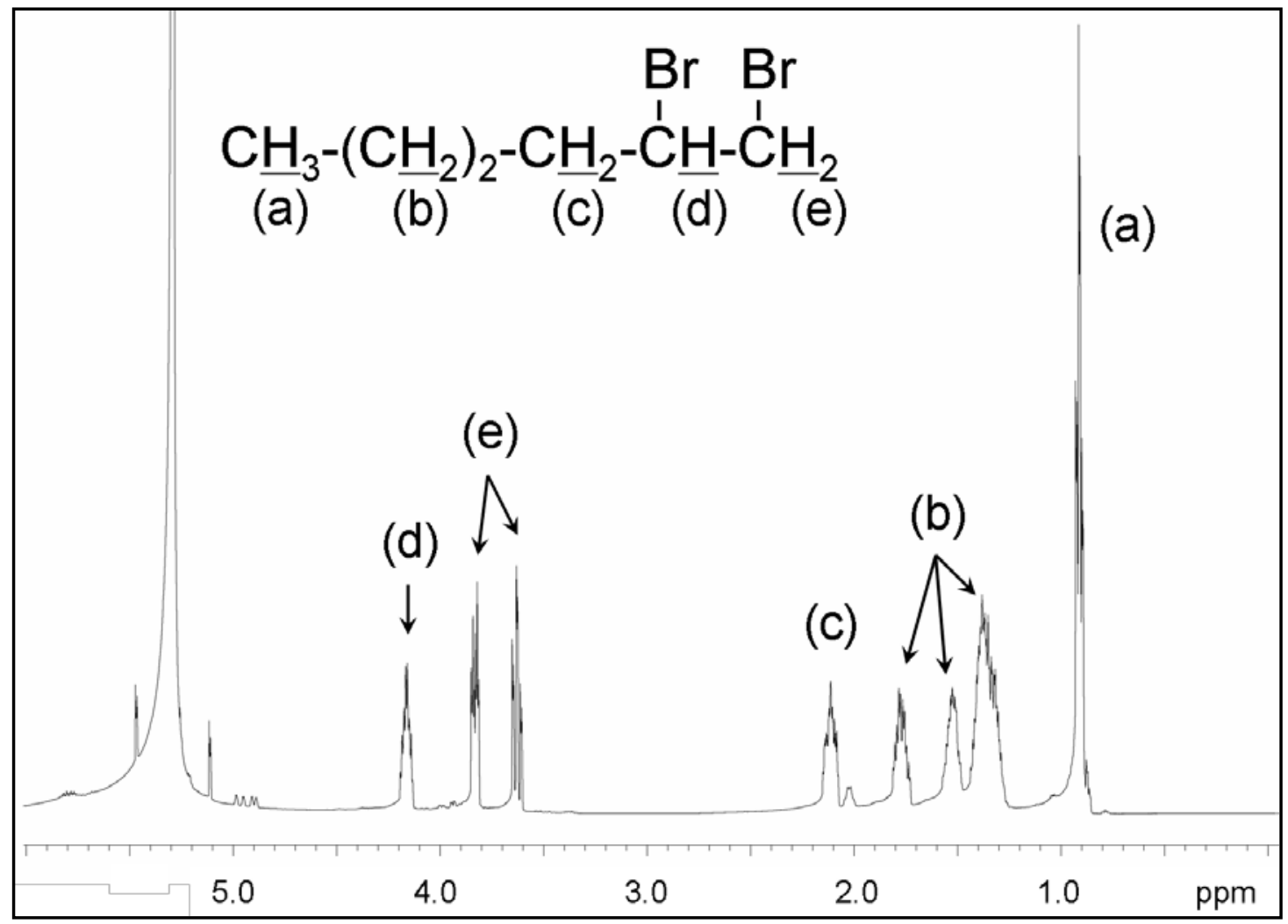

Figure 4-8: ${ }^{1} \mathrm{H}$ NMR spectrum of the reaction on the free molecules for experiment 4$1-3$ 
${ }^{1} \mathrm{H}$ NMR (500MHz, $\left.\mathrm{CDCl}_{3}\right)$

$\delta: 0.91 \mathrm{ppm}(3 \mathrm{H}$, ddd, H-(a), $J=3.2,7.3,17.3 \mathrm{~Hz}), \delta: 1.38 \mathrm{ppm}(2 \mathrm{H}, \mathrm{m}, \mathrm{H}-(\mathrm{b})), \delta$ : $1.52 \mathrm{ppm}(2 \mathrm{H}, \mathrm{m}, \mathrm{H}-(\mathrm{b})), \delta: 1.78 \mathrm{ppm}(2 \mathrm{H}, \mathrm{m}, \mathrm{H}-(\mathrm{b})), \delta: 2.11 \mathrm{ppm}(2 \mathrm{H}, \mathrm{m}, \mathrm{H}-(\mathrm{c}))$, $\delta$ : 3.63 ppm (1H, ddd, H-(e), J=2.7, 10.3, 19.8 Hz), $\delta: 3.82$ ppm (1H, dddd, H-(e), $J=1.0,4.6,10.3,14.6 \mathrm{~Hz}), \delta: 4.16 \mathrm{ppm}(1 \mathrm{H}, \mathrm{m}, \mathrm{H}-(\mathrm{d}))$

From the reaction on the free molecules, bromination of 1-hexene was clearly confirmed. The multiplet at $4.16 \mathrm{ppm}$ represents $\mathrm{H}$-(d). H-(e) signals were observed at $3.82 \mathrm{ppm}$ and $3.63 \mathrm{ppm}$ as split signals which probably because bromine atom has large electro negativity so it attracts other protons and restricts free rotation. H-(c) signals were seen at $1.78 \mathrm{ppm}$ and $2.11 \mathrm{ppm}$ also as split signals. The three protons of $\mathrm{H}$-(a) can be seen as a triplet at $0.91 \mathrm{ppm}$. The signals of $\mathrm{H}$-(b) were seen at $1.38 \mathrm{ppm}$. No signals indicating double bond from 1-hexene was seen in this spectrum which implies the success of bromination of 1-hexene. 


\section{Results of the reaction on the silicon quantum dots}

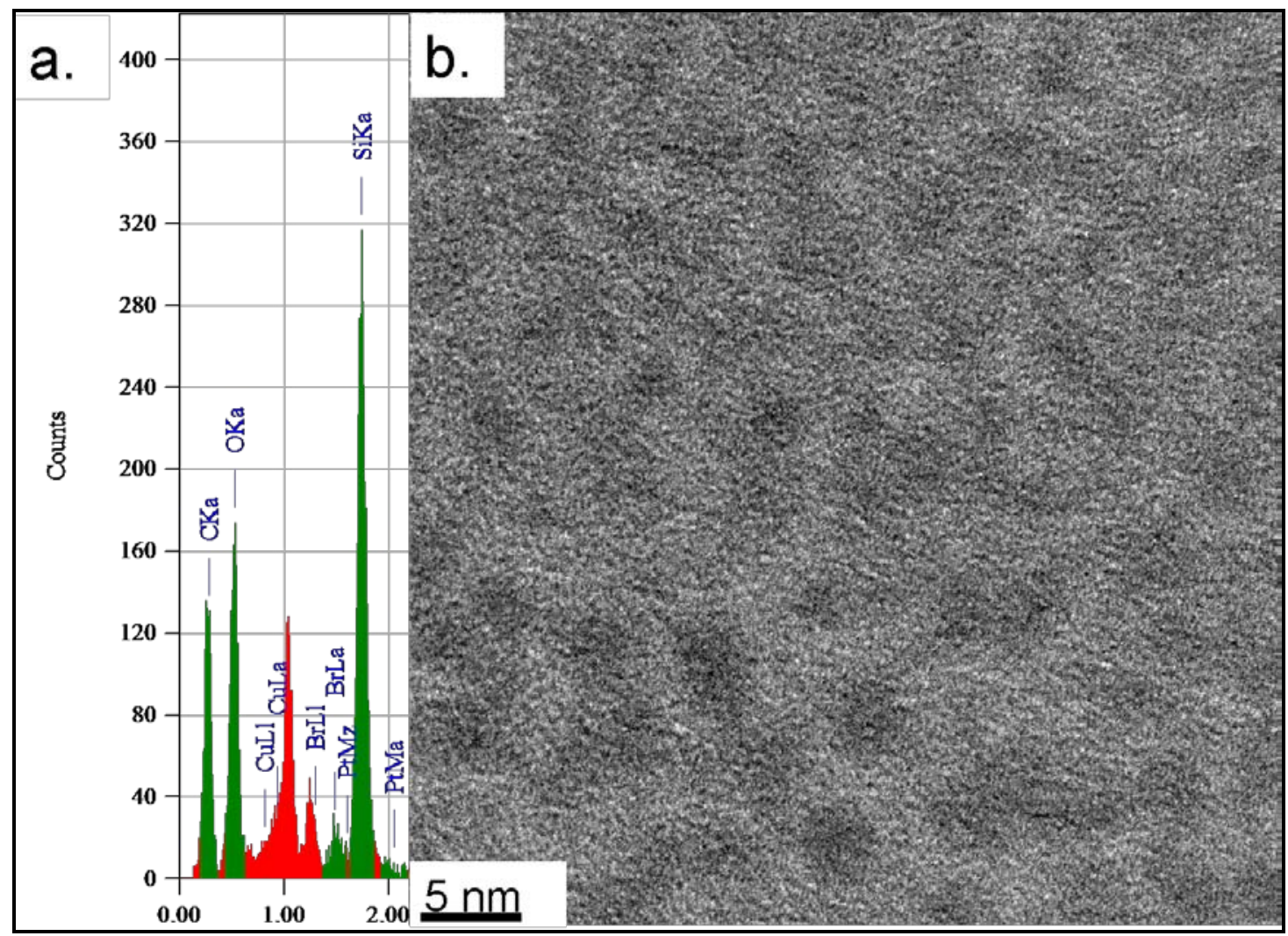

Figure 4-9: A TEM micrograph of silicon quantum dots obtained from experiment 4$1-3$.

As may be seen in Figure 4-9, nanoparticles were observed. They were nearly spherical and have an average diameter of $2.8 \pm 0.4 \mathrm{~nm}$. No high resolution TEM image was obtained because of the presence of impurities. SAED could not be obtained either from this sample. However since the silicon quantum dots capped with 1,5-hexadiene obtained from experiment 6 which was used for this experiment have been confirmed as crystalline silicon by SAED, these nanoparticles were possibly crystalline silicon as well.

EDS analysis showed the presence of silicon and bromine with the $\mathrm{K}_{\alpha}$ counts of 320 and $\mathrm{Ll}$ counts of 50 respectively and no platinum peak from the catalyst was observed.

Surface molecules were characterised by FTIR and ${ }^{1} \mathrm{H}$ NMR spectra. C-Br stretching can be seen at around $580 \mathrm{~cm}^{-1}$ in FTIR spectrum. ${ }^{7}$ Also $\mathrm{CH}_{2}$ vibration of $\mathrm{CH}_{2}-\mathrm{Br}$ group was seen at $1115 \mathrm{~cm}^{-1}$. The peaks at $1260 \mathrm{~cm}^{-1}$ and $1460 \mathrm{~cm}^{-1}$ represent $\mathrm{Si}-\mathrm{CH}_{2}$ vibrational scissoring. The peak from $2850 \mathrm{~cm}^{-1}$ to $2955 \mathrm{~cm}^{-1}$ indicates carbon chain 
of the surface moieties. Since the peak corresponding to the double bond at over 3000 $\mathrm{cm}^{-1}$ was not observed the terminal double bonds on the surface moieties were successfully brominated.

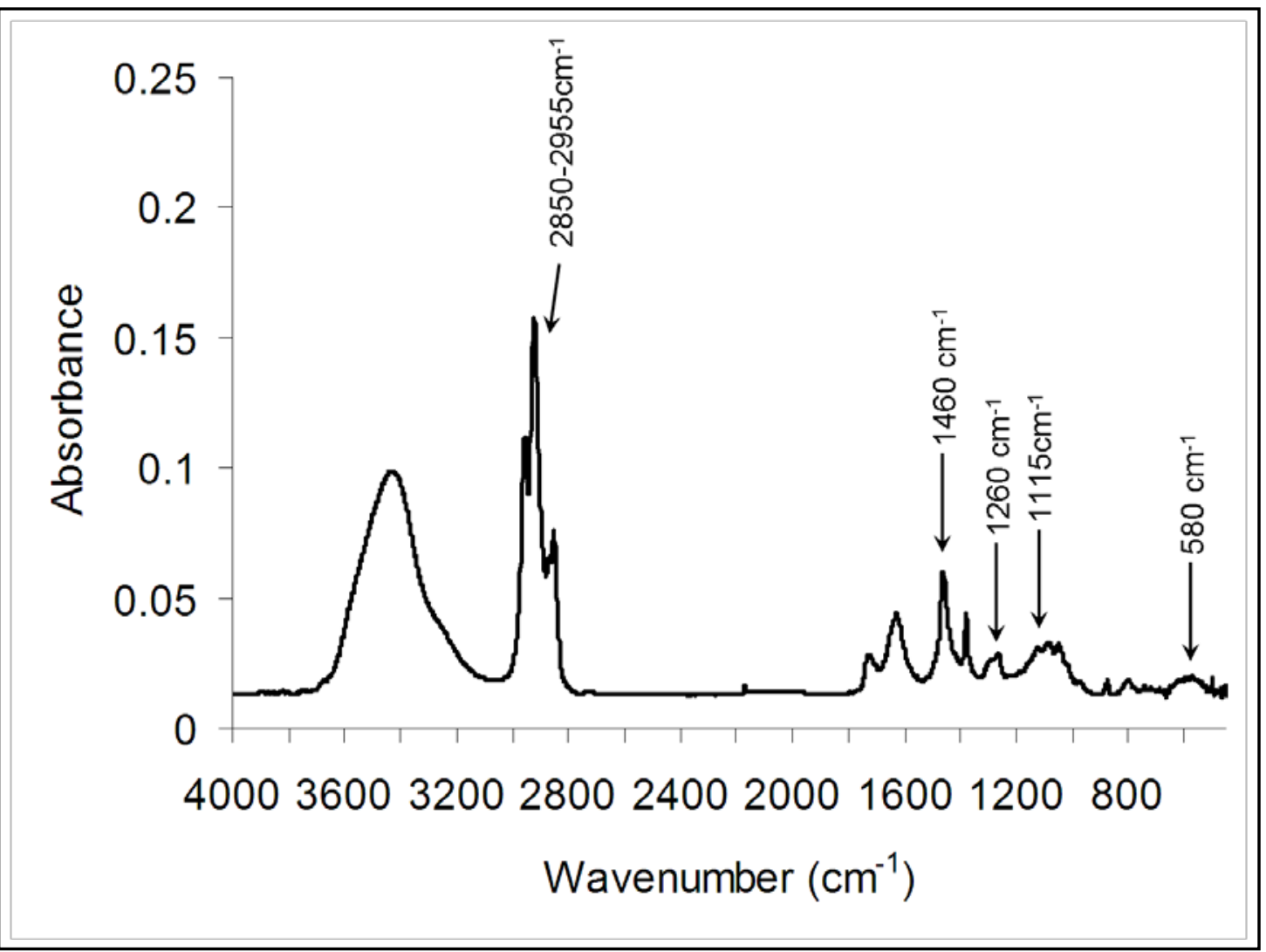

Figure 4-10: FTIR spectrum of silicon quantum dots obtained from experiment 4-1-3

In ${ }^{1} \mathrm{H}$ NMR spectrum, H-(d) signal was observed at $4.09 \mathrm{ppm}$ as a broad signal because of the bromine atom. Theoretically C-C single bond is free rotation, however bromine atom is slightly electron negative which attracts the protons near by (H-(c), (d), (e)). This will restricts the C-C bond free rotation. H-(e) protons were observed at $3.66 \mathrm{ppm}$ and $3.78 \mathrm{ppm}$. As described in the experiment on the free molecules, usually the carbon-carbon single bond has free rotation however the bromine atom is big and also has large electro negativity. Therefore the bromide atom at the terminal end of the surface moiety interacts with the protons around the atom. As a result, the carbon-carbon bond free rotation was restricted, giving different chemical environments for $\mathrm{H}$-(e) protons and thus different chemical shifts. No signals 
corresponding to the double bond at the terminal end of the surface moieties, 4.90 ppm and 5.73 ppm, were observed in this spectrum.

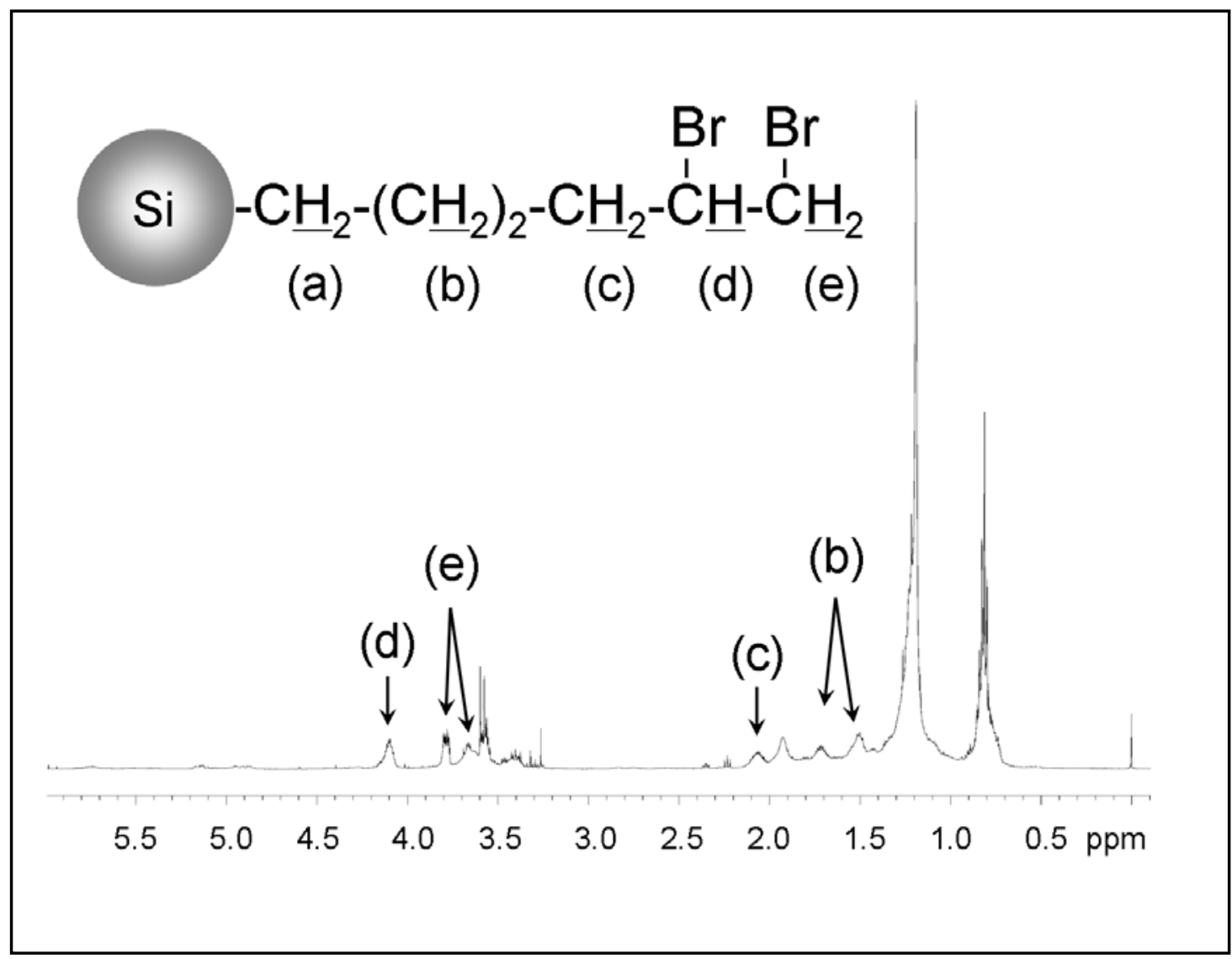

Figure 4-11: ${ }^{1} \mathrm{H}$ NMR spectrum of silicon quantum dots obtained from experiment 4$1-3$

${ }^{1} \mathrm{H}$ NMR (500MHz, $\left.\mathrm{CDCl}_{3}\right)$

$\delta$ : 3.66 ppm (1H, s, H-(e)), $\delta: 3.78$ ppm (1H, m, H-(e)), $\delta: 4.09$ ppm (1H, s, H-(d))

From the FTIR and the ${ }^{1} \mathrm{H}$ NMR spectra, there was no signal of remaining of the double bond at the terminal end of the surface moieties. The results support the successful bromination of the double bonds at the terminal end of the surface moieties of the silicon quantum dots. 


\section{Titrations}

To find out the yield of the silicon quantum dots, the titration of the silicon quantum dots with $0.05 \%$ (vol /\%) bromine solution in dichloromethane was carried out.

\section{Titration results}

Silicon quantum dot capped with 1,5-hexadiene/ $0.05 \%$ bromine solution

\begin{tabular}{|l|l|l|l|}
\hline Initial & Final & Total & Mean titre \\
\cline { 1 - 3 } $35.03 \mathrm{~mL}$ & $35.60 \mathrm{~mL}$ & $0.57 \mathrm{~mL}$ & \multirow{2}{*}{$0.57 \mathrm{~mL}$} \\
\cline { 1 - 2 } $35.60 \mathrm{~mL}$ & $36.20 \mathrm{~mL}$ & $0.60 \mathrm{~mL}$ & \\
\cline { 1 - 2 } $36.25 \mathrm{~mL}$ & $37.05 \mathrm{~mL}$ & $0.55 \mathrm{~mL}$ & \\
\hline
\end{tabular}

\section{Calculation}

The mole of bromine in $0.57 \mathrm{~mL}$ of $0.05 \%$ bromine solution is 0.000562 moles. One mole of bromine will react with one mole of double bond therefore the total amount of the double bond on the surface of silicon quantum dots are 0.000562 moles.

The yield of silicon quantum dot was calculated based on the reference below. ${ }^{11}$

\begin{tabular}{|l|l|l|}
\hline Diameter & $d=2.0 \mathrm{~nm}$ & $d=5.0 \mathrm{~nm}$ \\
\hline Surface area $\left(\mathrm{nm}^{2}\right) 4 \pi \mathrm{r}^{2}$ & 50.27 & 314.16 \\
\hline Number of atoms on the surface & 139 & 868.75 \\
\hline Volume $\left(\mathrm{nm}^{3}\right) 4 / 3 \pi \mathrm{r}^{3}$ & 33.51 & 523.6 \\
(number of silicon atoms) & $(199)$ & $(3109)$ \\
\hline
\end{tabular}

The amount of double bond

0.000562 moles $=3.3485 \times 10^{20}$ (Numbers of the double bonds reacted with bromine)

The amount of silicon quantum dots

$3.3485 \times 10^{20} / 868.75$ (number of the atoms on the surface) $=3.854 \times 10^{17}$

$\left(6.4 \times 10^{-7}\right.$ moles $)$

The amount of silicon precursor used in this experiment

$\mathrm{SiBr}_{4}(300 \mu \mathrm{L})=0.0024$ moles

The amount of silicon atoms

0.0024 molesx6.02 $\times 10^{23}$ (Avogadro Constant) $=1.45 \times 10^{21}$ (the total number of the silicon atoms used in the reaction) 
The amount of silicon quantum dots

$\left(1.4448 \times 10^{21}\right) / 3109$ (the number of the silicon atoms / quantum dot) $=4.647 \times 10^{17}$ (7.71 $1 \times 10^{-7}$ moles)

Theoretical yield : $\left[\left(6.4 \times 10^{-7}\right) /\left(7.71 \times 10^{-7}\right)\right] \times 100=83.0 \%$

\section{Experimental}

Silicon quantum dots capped with 1,5-hexadiene from experiment 6 from chapter 3 was dissolved in $2 \mathrm{~mL}$ of dichloromethane. $3 \%$ of bromine solution in dichloromethane was added dropwise from burette to the silicon quantum dot's solution till the very light bromine colour by eye remained in the solution. The solution was dried up under vacuum and re-dissolved in dichloromethane.

\section{Experiment 4-1-4: Conversion of the terminal double bond of silicon quantum dots to an alcohol functional group}

Silicon quantum dots capped with 1-hexanol were synthesised from silicon quantum dots capped with 1,5-hexadiene obtained from experiment 6. Alcohol group has less steric hindrance compared to diol groups which makes it easier to attach bigger molecules such as peptides or antibodies. The hydroxyl group on the surface of the silicon quantum dots will make them water soluble and can broaden the possible range of biocompatible molecules, which can be attached to the surface. The hydroboration oxidation reaction was chosen to convert the double bond to the hydroxyl group. The reaction was confirmed by using 1-hexene and characterised by ${ }^{1} \mathrm{H}$ NMR analysis.

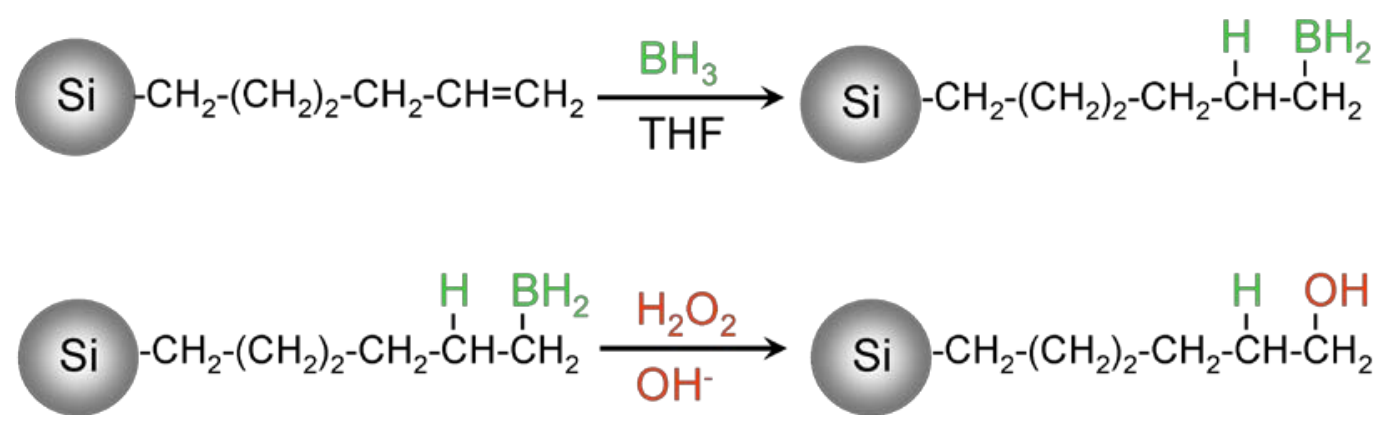

Reaction scheme of hydroboration oxidation on the surface of silicon quantum dot 


\section{${ }^{1} \mathrm{H}$ NMR result of the reaction on the free molecules}

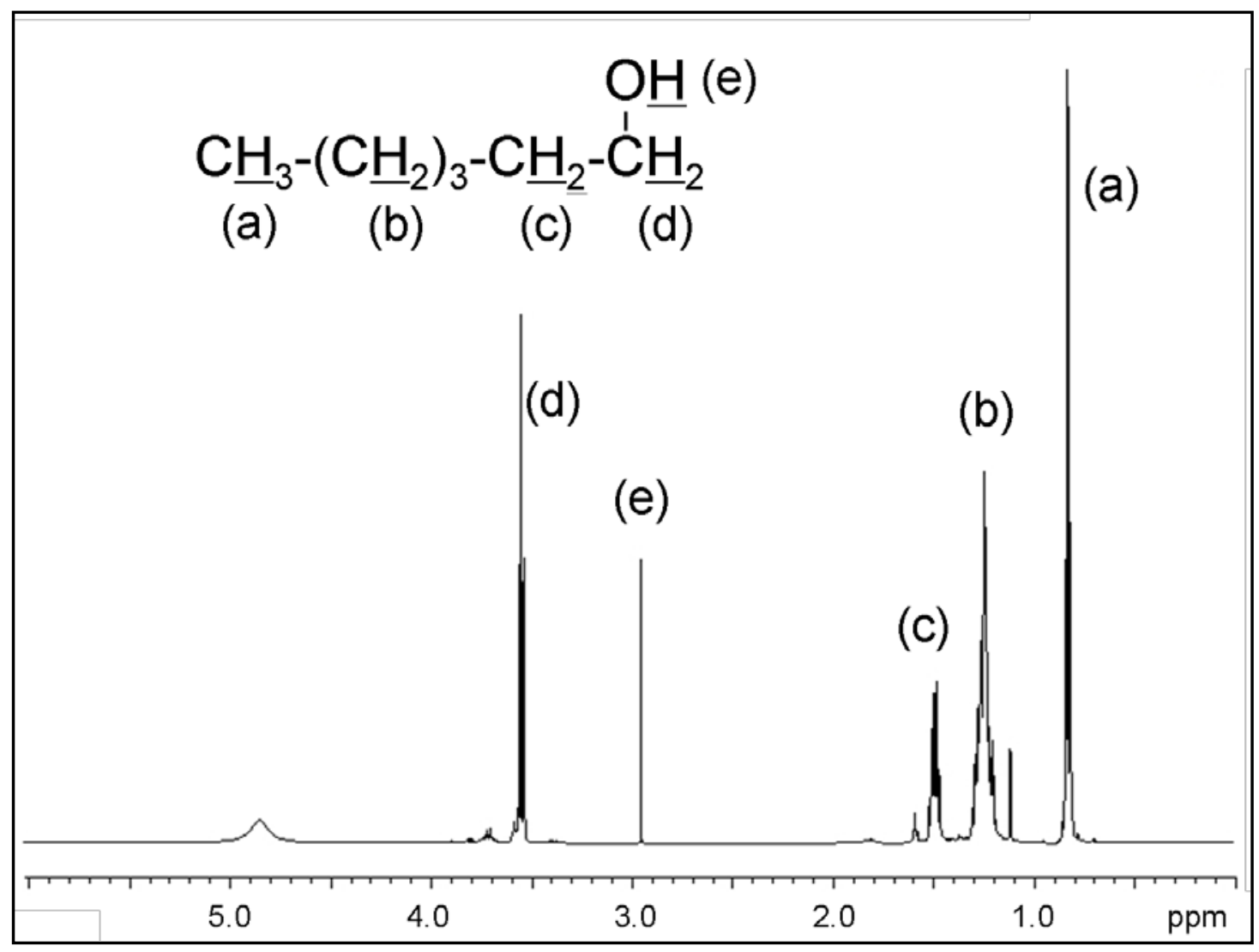

Figure 4-12: ${ }^{1} \mathrm{H}$ NMR spectrum of the reaction on the free molecules for experiment 4-1-4

${ }^{1} \mathrm{H}$ NMR (500MHz, $\left.\mathrm{CDCl}_{3}\right)$

$\delta: 0.83$ ppm (3H, t, H-(a) J=6.8 Hz), $\delta: 1.24$ ppm (6H, m, H-(b)), $\delta: 1.50$ ppm (2H, m, H-(c)), $\delta: 2.95$ ppm (1H, s, H-(e)), $\delta: 3.55$ ppm (2H, t, H-(d), J=6.8 Hz)

Following the reaction on the free molecules, a very clear ${ }^{1} \mathrm{H}$ NMR spectrum of 1hexanol was seen. The triplet integrating for three protons at $0.83 \mathrm{ppm}$ represents the three protons of $\mathrm{H}-(\mathrm{a})$. The multiplet seen at $1.24 \mathrm{ppm}$ which integrates for six protons corresponds to H-(b). The signal from $\mathrm{H}-(\mathrm{c})$ was seen at $1.50 \mathrm{ppm}$ as a multiplet. The signal at $3.55 \mathrm{ppm}$ integrates for two protons which represent $\mathrm{H}-(\mathrm{d})$. (data not shown) The signal at $2.95 \mathrm{ppm}$ integrates for one proton which indicates the proton of the hydroxyl group (H-(e)). 


\section{Results of the reaction on the silicon quantum dots}

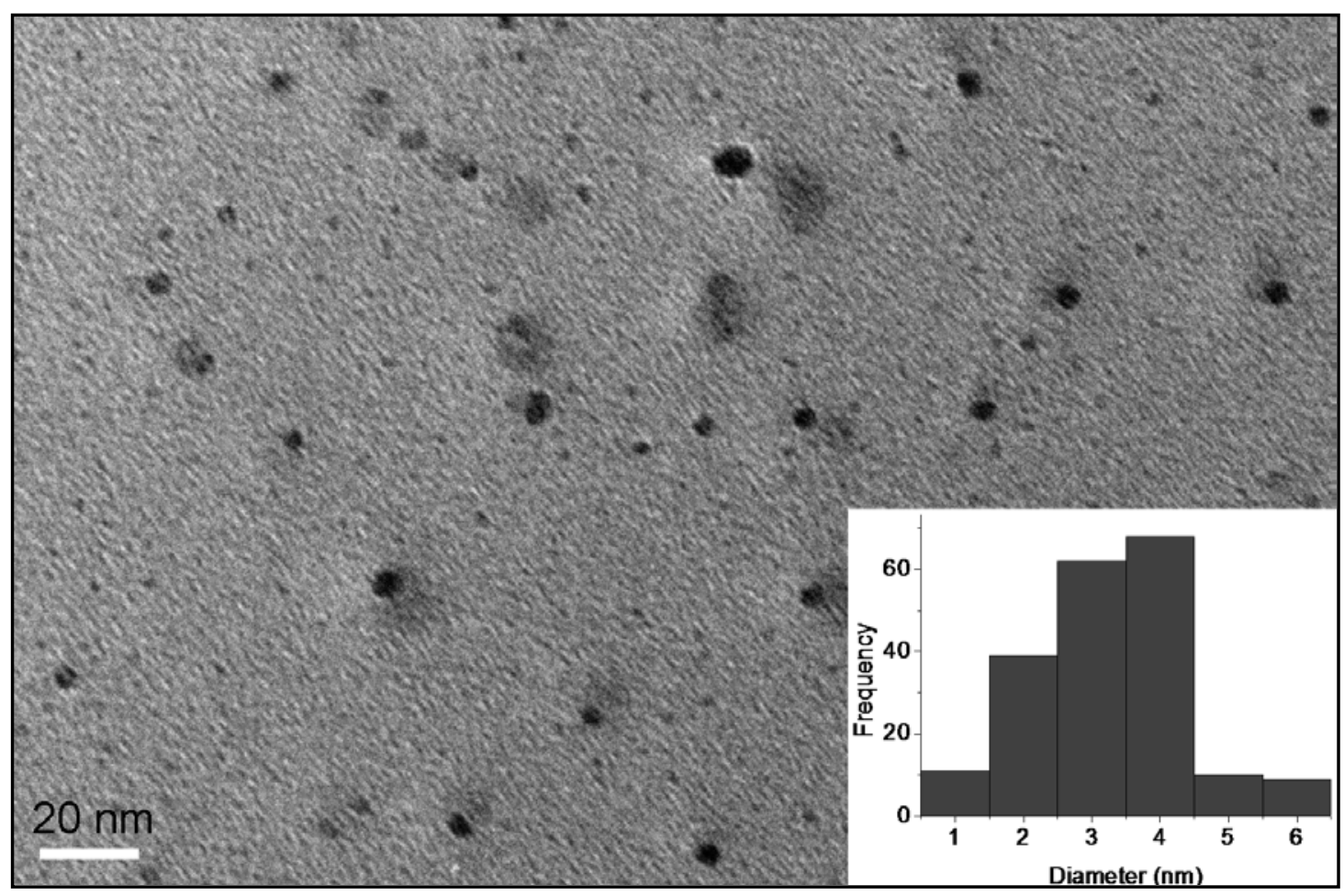

Figure 4-6: A TEM micrograph of silicon quantum dots obtained from experiment 4$1-4$

The results of the hydroboration of the 1,5-hexadiene capped silicon quantum dots are given in Figure 4-13. As may be seen in Figure 4-13, spherical nanoparticles were observed by TEM. The nanoparticles were poly dispersed and majority of the particles had an average size of $3.7 \pm 1.1 \mathrm{~nm}$. However some of the nanoparticles had bigger size around $15 \mathrm{~nm}$. SAED and lattice fringes could not be obtained to confirm as crystalline silicon however the surface modification was conducted on the nanoparticles from experiment 6 in which the nanoparticles were confirmed as crystalline silicon. EDS analysis also showed the presence of silicon and no peak of platinum from the platinum catalyst was observed. It indicates the nanoparticles were composed mainly of silicon. 


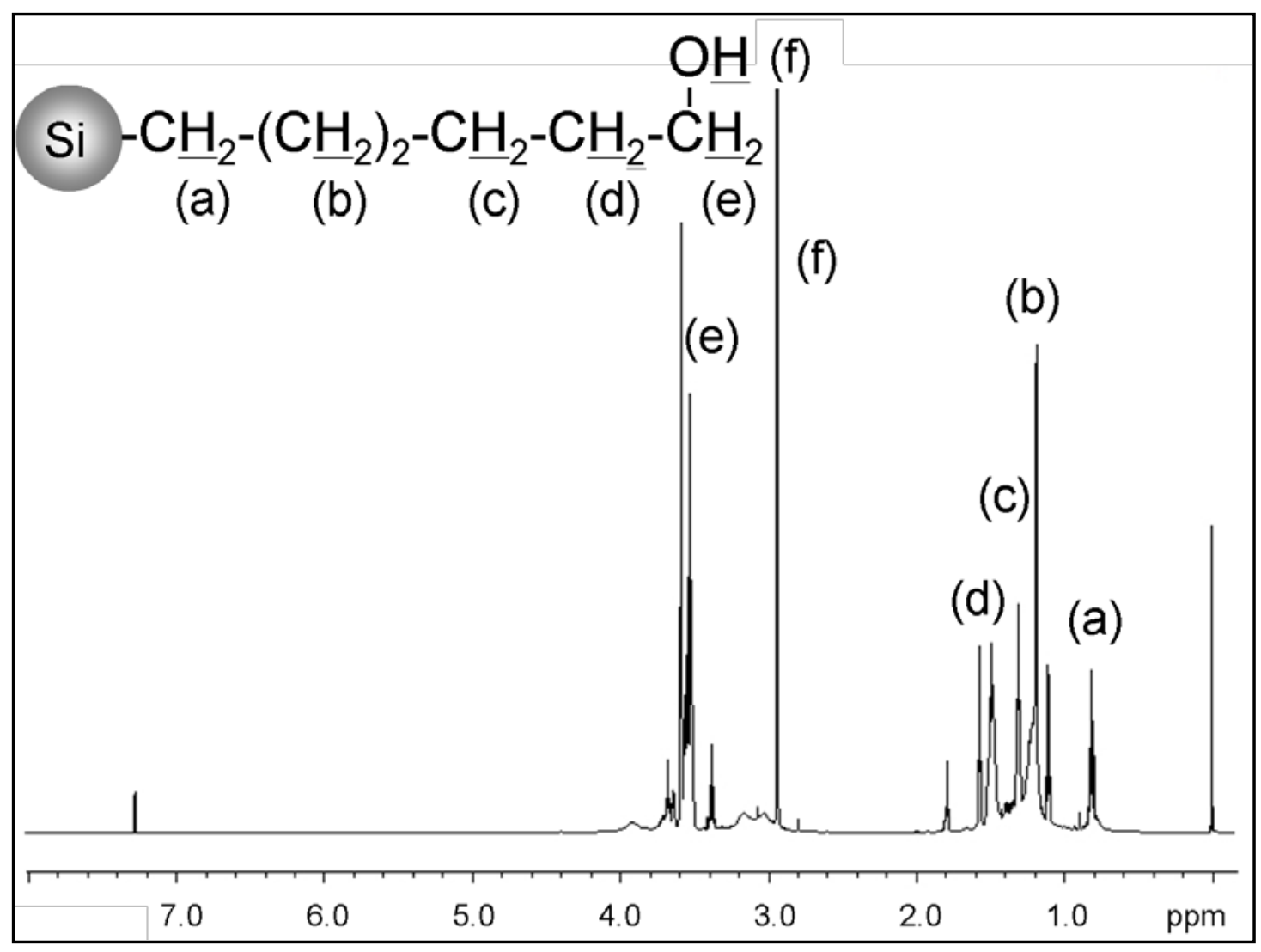

Figure 4-7: A ${ }^{1} \mathrm{H}$ NMR spectrum of silicon quantum dots obtained from experiment 4-1-4

${ }^{1} \mathrm{H}$ NMR (500MHz, $\mathrm{CDCl}_{3}$ )

$\delta: 0.81 \mathrm{ppm}(2 \mathrm{H}, \mathrm{t}, \mathrm{H}-(\mathrm{a}) J=5.6 \mathrm{~Hz}), \delta: 1.19 \mathrm{ppm}(4 \mathrm{H}, \mathrm{m}, \mathrm{H}-(\mathrm{b})), \delta: 1.31 \mathrm{ppm}(2 \mathrm{H}$, m, H-(c)), $\delta: 1.49$ ppm (2H, q, H-(d), J=6.1), $\delta: 2.93$ ppm (1H, s, H-(f)), $\delta: 3.53$ ppm (2H, m, H-(e))

The surface molecules were characterised by ${ }^{1} \mathrm{H}$ NMR analysis. The signal from the two protons, $\mathrm{H}-(\mathrm{e})$, was seen at $3.53 \mathrm{ppm}$ as a multiplet. Those two protons are less shielded compared to other protons. H-(d) and H-(c) protons can be seen at $1.49 \mathrm{ppm}$ and $1.31 \mathrm{ppm}$ respectively. The four protons of the $\mathrm{H}$-(b) were observed at $1.19 \mathrm{ppm}$ as a multiplet. The triplet at $0.81 \mathrm{ppm}$ was characterised as $\mathrm{H}-(\mathrm{a})$ protons. However the integrals did not match to the expected numbers of protons, this could be due to the overlapping of the peaks from impurities and surfactant, $\mathrm{C}_{12} \mathrm{E}_{5}$. 


\section{Experimental}

Silicon quantum dots capped with 1,5-hexadiene from experiment 6 were dissolved in $5 \mathrm{~mL}$ of tetrahydrofuran (THF) on an ice water bath. $0.1 \mathrm{~mL}$ of borane-dimethyl sulphide (2M in THF) was added dropwise to the solution while stirring. After stirring for 5 hours, $2 \mathrm{~mL}$ of $10 \% \mathrm{NaOH}$ solution was added dropwise followed by $2 \mathrm{~mL}$ of $30 \%$ hydrogen peroxide solution were added carefully. After stirring for another 16 hours the reaction mixture was diluted with diethyl ether and the aqueous phase was saturated with sodium chloride. The organic layer was extracted with diethyl ether three times and the extracts were dried over $\mathrm{MgSO}_{4}$.

\section{Experiment 4-1-5: Oxidation of terminal alcohol of silicon quantum dots to carboxylic acid}

After the hydroboration reaction of the terminal double bond of the silicon quantum dots, a further reaction to convert the hydroxyl group to a carboxyl group was conducted. Chromic trioxide was used as an oxidizing agent in sulphuric acid. The reaction is known as the Jones oxidation. ${ }^{12}$ Silicon quantum dots obtained from experiment 4-1-4 were used for this trial. The terminal carboxyl group can form a peptide bond with an amine group which broadens the possibilities of attaching biocompatible molecules to the surface.

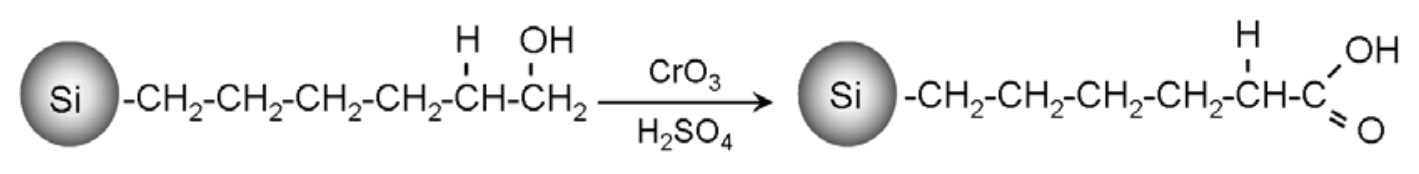

\section{Reaction scheme of Jones oxidation on silicon quantum dots}

\section{Results}

A reaction on the free molecules and the reaction using the product produced from the hydroboration reaction on silicon quantum dots capped with 1,5-hexadiene were conducted. The both solutions were characterized by ${ }^{1} \mathrm{H}$ NMR. In the spectrum of the silicon quantum dots after the Jones oxidation, the signal at $3.53 \mathrm{ppm}$ observed from the experiment on the free molecules of 4-1-4 had disappeared, however no signal was observed around $2.40 \mathrm{ppm}$ where protons attached to the carbon next to carboxyl 
group which might be expected to produce a signal. A signal integrating for one proton was observed at $1.93 \mathrm{ppm}$. Three signals observed at $1.63 \mathrm{ppm}, 1.19 \mathrm{ppm}$ and $0.81 \mathrm{ppm}$ were in the places where $\mathrm{H}-(\mathrm{c}), \mathrm{H}-(\mathrm{b})$ and $\mathrm{H}-(\mathrm{a})$ were expected. Since H-(d) could not be characterized properly, the success of this reaction could not be confirmed. A possible reason for this result might be due to an effect of the strong acidic conditions on the reagents.

\section{Experiment 4-1-6: Biotinylation of terminal amine group of silicon quantum dots}

Biotin terminated silicon quantum dots were synthesised from the terminal amine group on the surface from experiment 8 from chapter 3 . Biotin is also known as vitamin $\mathrm{H}$ or $\mathrm{B} 7$. It is a water soluble B-complex vitamin which is composed of a tetrahydroimidizalone ring fused with tetrahydrothiophene ring. A valeric acid substituent is attached to one of the carbon atoms of the tetrahydrothiophene ring. ${ }^{13}$ In this experiment, for the biotinylation of the silicon quantum dots, the biotin compound, sulfo-LC-NHS-biotin which has a spacer arm with terminal succinimide group was used as a reagent. The structure of biotin and sulfo-LC-NHS-biotin are given in Figure 4-15.

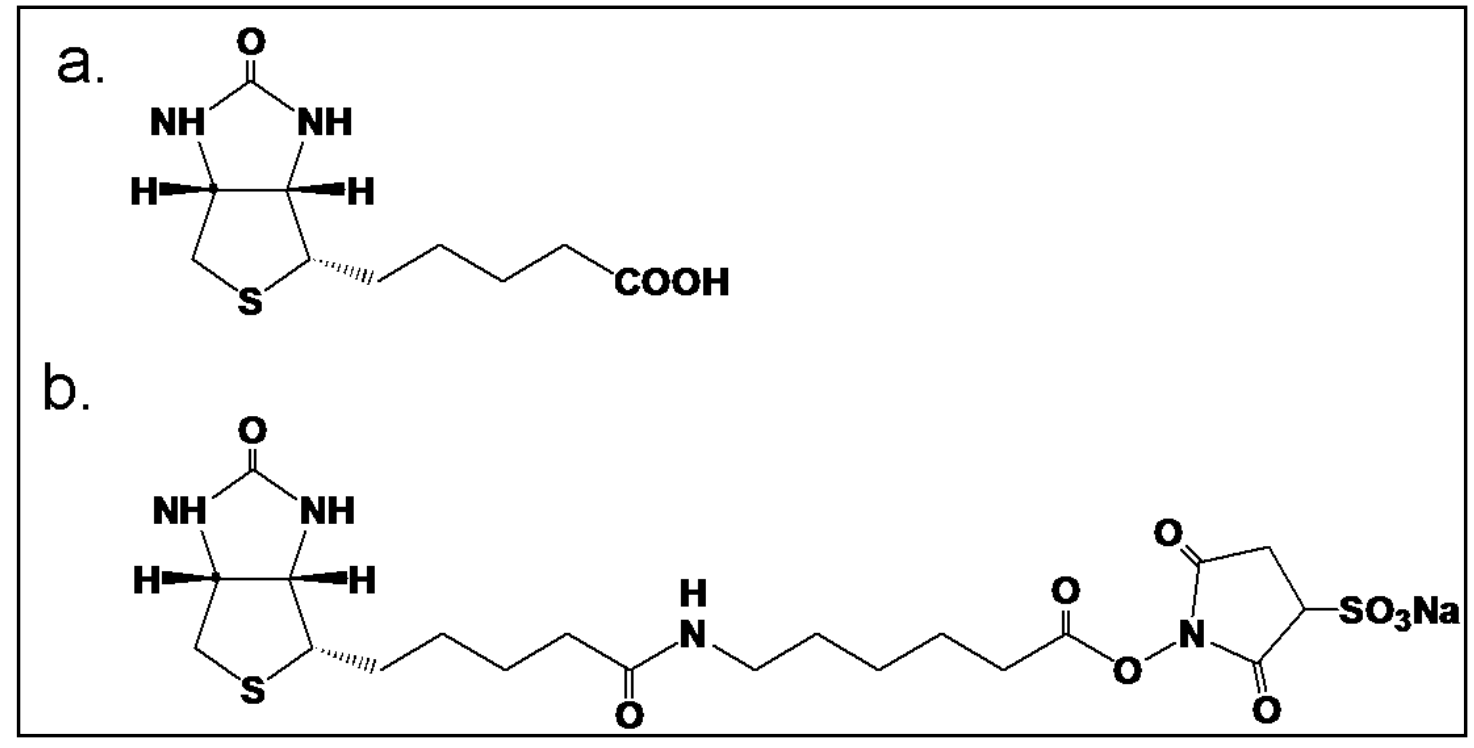

Figure 4-8: (a) the structure of biotin: (b) the structure of sulfo-LC-NHS-biotin 
Since biotin is known to bind to avidins with a high degree of affinity specificity, the biotin-avidin system is widely used as a powerful tool in biological science. ${ }^{14,15}$ Avidin is a tetrameric protein and contains four identical subunits, each of which binds to biotin, and therefore biotin-tagged molecules can easily be attached to drug molecules or antibodies through avidins. Since succinimide is a good leaving group the terminal amine group on the silicon quantum dots can bind to the carbon atom of the ketone part.

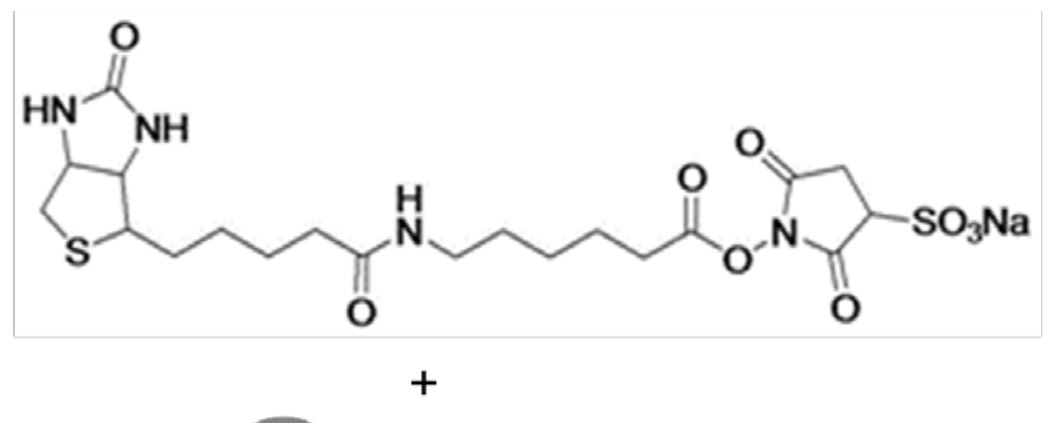

Si $-\mathrm{CH}_{2}-\mathrm{CH}_{2}-\mathrm{CH}_{2}-\mathrm{NH}_{2}$

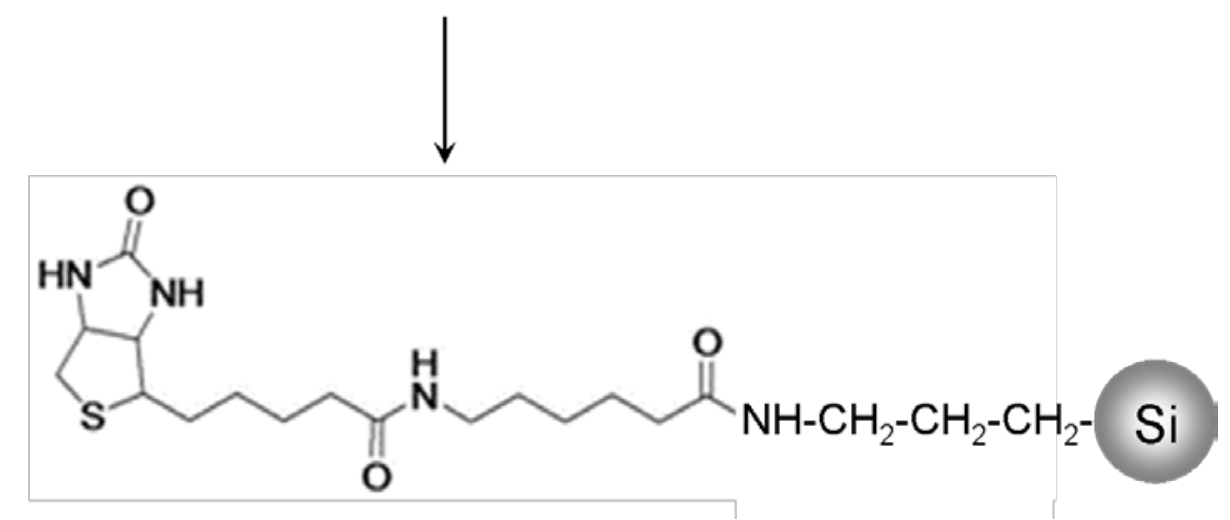

\section{Reaction scheme of the biotinylation of silicon quantum dot}

The reaction on the free molecules was conducted with tert-butylamine and 9fluorenylmethyl succinimidyl carbonate to check the reaction between succinimide group and amine group. 9-fluorenylmethyl succinimidyl carbonate was chosen for the free molecules reaction since the product has most of the protons signals above 4.0 
ppm in ${ }^{1} \mathrm{H}$ NMR which makes it easier to see the chemical shift of the succinmide group compared to sulfo-LC-NHS-biotin. Also it is easier to obtain large quantity with a reasonable price compared to sulfo-LC-NHS-biotin. The reaction was run in tetrahydrofuran (THF) and dimethylaminopyridine (DMAP) was used as a catalyst. The reaction mixture was characterised by ${ }^{1} \mathrm{H}$ NMR spectrum.

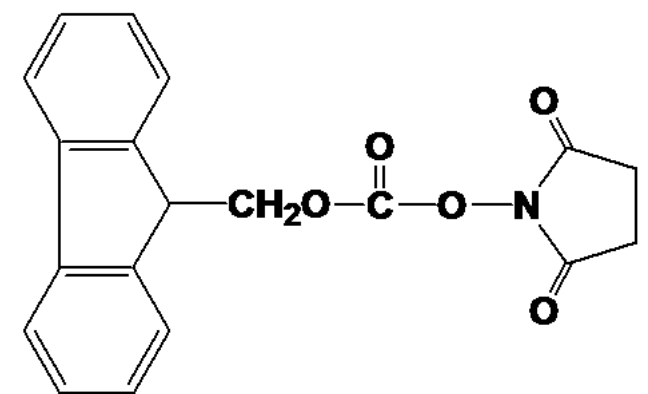

\section{9-fluorenylmethyl succinimidyl carbonate}

\section{${ }^{1} \mathrm{H}$ NMR result of the reaction on the free molecules}

In the spectrum of free 9-fluorenylmethyl succinimidyl carbonate, a sharp signal corresponding to the protons of the succinimide group can be seen at $2.85 \mathrm{ppm}$. This signal had disappeared after the reaction with tert-butylamine, and a new multiplet was observed around $1.40 \mathrm{ppm}$ which indicates the replacement of succinimide group by tert-butylamine. The rest of the peaks remained almost the same. (Figure 4-16 (a), (b)) 


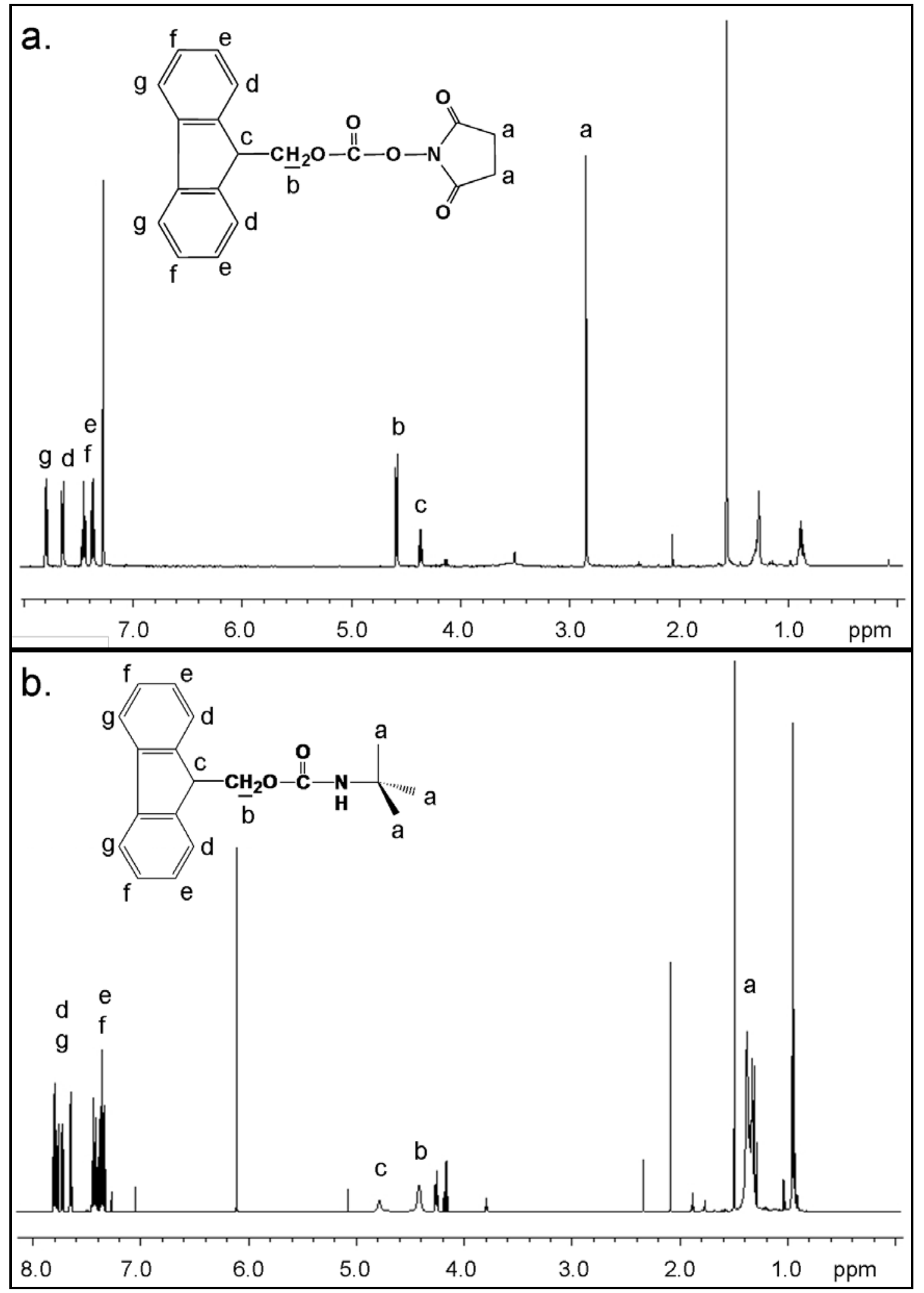

Figure 4-9: ${ }^{1} \mathrm{H}$ NMR spectrum of (a): 9-fluorenylmethyl succinimidyl carbonate (b): after the reaction with tert-butylamine. 
Free 9-fluorenylmethyl succinimidyl carbonate

${ }^{1} \mathrm{H}$ NMR (500MHz, $\left.\mathrm{CDCl}_{3}\right)$

$\delta: 2.83$ ppm (4H, s, H-(a)), $\delta: 4.35$ ppm (1H, t, H-(c), J=7.1 Hz), $\delta: 4.56$ ppm (2H, d, H-(b), J=7.3 Hz), $\delta: 7.35$ ppm (2H, t, H-(d), J=7.3 Hz), $\delta: 7.43$ ppm (2H, t, H-(e), $J=7.3 \mathrm{~Hz}$ ), $\delta: 7.61 \mathrm{ppm}(2 \mathrm{H}, \mathrm{d}, \mathrm{H}-(\mathrm{f}), J=7.6 \mathrm{~Hz}), \delta: 7.77 \mathrm{ppm}$ (2H, d, H-(g), $J=7.3$ $\mathrm{Hz})$

The product after the reaction with tert-butylamine

${ }^{1} \mathrm{H}$ NMR (500MHz, $\left.\mathrm{CDCl}_{3}\right)$

$\delta: 1.32$ ppm (9H, m, H-(a)), $\delta: 4.40$ ppm (2H, s, H-(b)), $\delta: 4.77$ ppm (1H, s, H-(c)),

$\delta: 7.35$ ppm (2H, dd, H-(e), J=1.3, 2.5 Hz), $\delta: 7.40$ ppm (2H, dd, H-(f), J=1.3, 7.6

Hz), $\delta: 7.63$ ppm (2H, d, H-(d), J=7.3 Hz), $\delta: 7.78$ ppm (2H, d, H-(g), J=7.6 Hz)

\section{Results of the reaction on the silicon quantum dots}

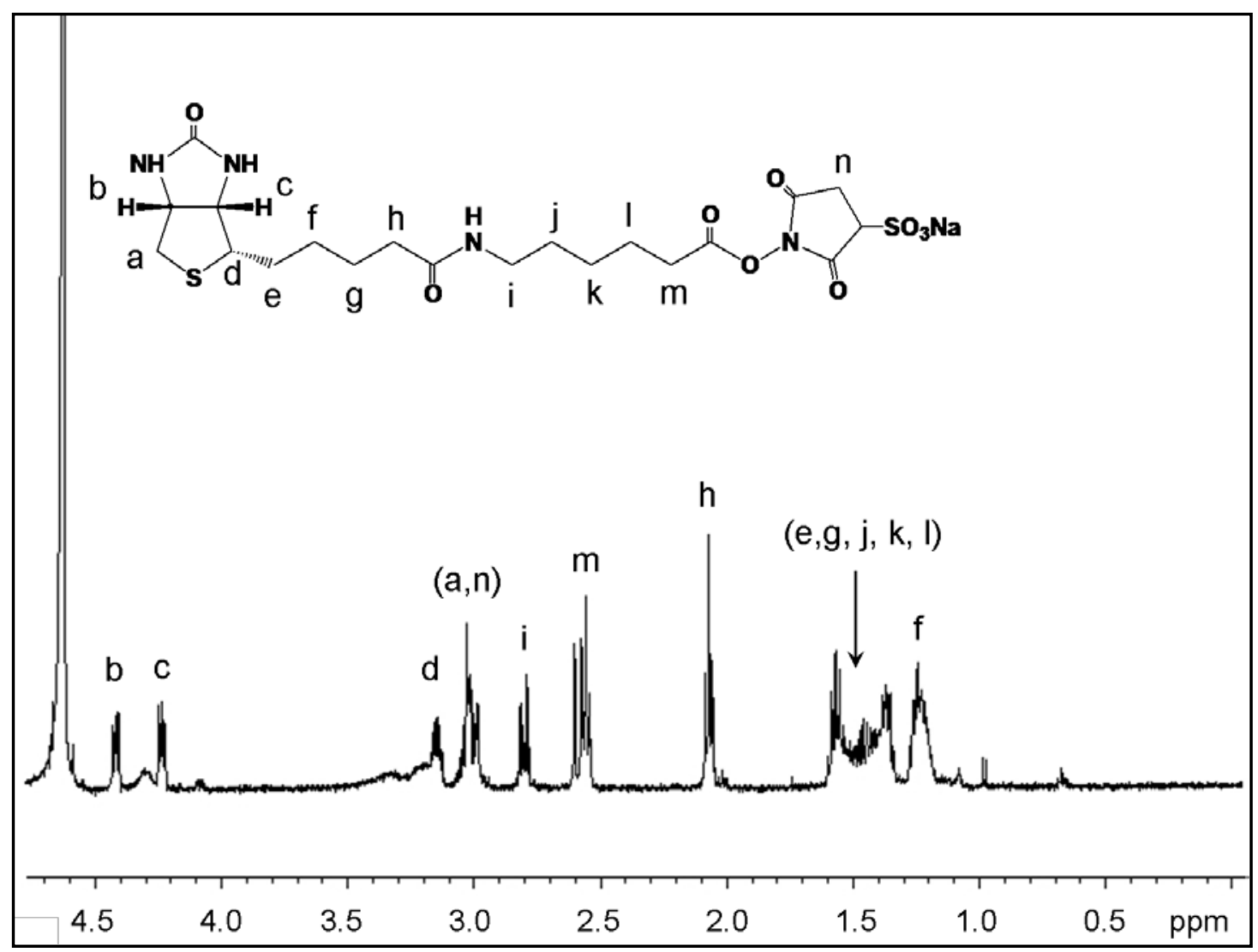

Figure 4-10: ${ }^{1} \mathrm{H}$ NMR spectrum of free sulfo-LC-NHS-biotin. 


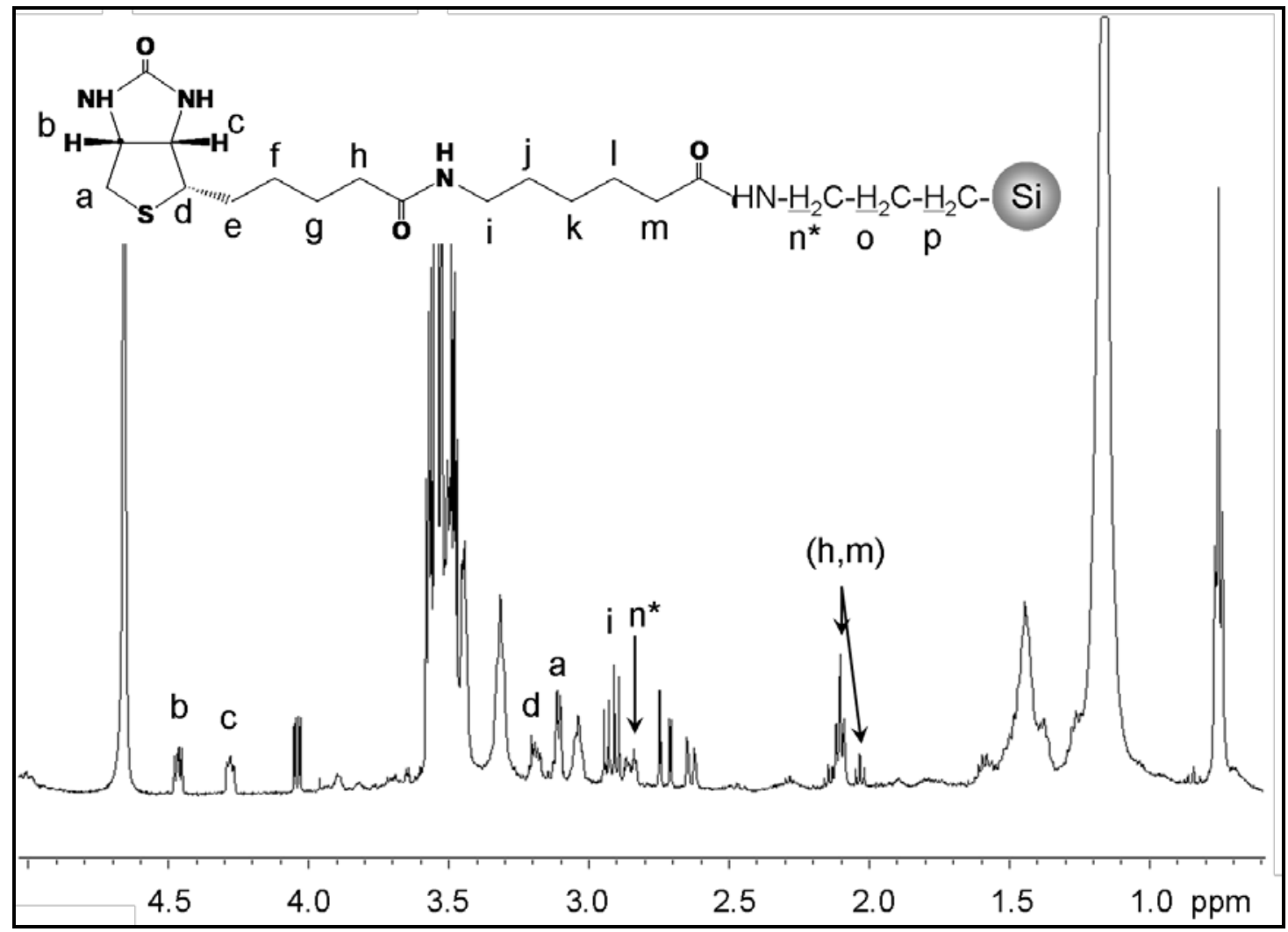

Figure 4-11: ${ }^{1} \mathrm{H}$ NMR spectrum after the reaction with the silicon quantum dot capped with allylamine.

Free sulfo-LC-NHS-biotin

${ }^{1} \mathrm{H}$ NMR (500MHz, $\mathrm{CDCl}_{3}$ )

$\delta: 1.23$ ppm (2H, m, H-(f)), $\delta: 2.07$ ppm (2H, t, H-(h), J=7.1 Hz), $\delta: 2.54$ ppm (2H, t, H-(m), J=8.8 Hz), $\delta: 2.80$ ppm (2H, dd, H-(i), J=4.9, $13.0 \mathrm{~Hz}), \delta: 3.0 .3 \mathrm{ppm}(4 \mathrm{H}$, m, H-(a),(n)), $\delta: 3.14$ ppm (1H, m, H-(d)), $\delta: 4.22$ ppm (1H, dd, H-(c), J=4.6, 8 Hz), $\delta: 4.41 \mathrm{ppm}(1 \mathrm{H}, \mathrm{dd}, \mathrm{H}-(\mathrm{b}), J=4.4,7.8 \mathrm{~Hz})$

The product after the reaction with the silicon quantum dots capped with allylamine ${ }^{1} \mathrm{H}$ NMR (500MHz, $\mathrm{CDCl}_{3}$ )

$\delta: 2.03$ ppm (2H, t, H-(m), J=7.6 Hz), $\delta: 2.10$ ppm (2H, t, H-(h), J=7.1 Hz), $\delta$ : 2.83 ppm (2H, dd, H-(n*)), $\delta: 2.90$ ppm (2H, dd, H-(i), J=8.3, $18.3 \mathrm{~Hz}), \delta: 3.11$ ppm (2H, d, H-(a), J=5.4 Hz), $\delta: 3.20$ ppm (1H, m, H-(d)), $\delta: 4.28$ ppm (1H, t, H(c)), $\delta: 4.46 \mathrm{ppm}(1 \mathrm{H}, \mathrm{m}, \mathrm{H}-(\mathrm{b}))$ 
The biotinylation of the silicon quantum dot was conducted by mixing the amine terminated silicon quantum dots and sulfo-LC-NHS-biotin. After the reaction, the sample was characterised by ${ }^{1} \mathrm{H}$ NMR analysis. From the result, two triplets signals were observed at $2.02 \mathrm{ppm}$ and $2.10 \mathrm{ppm}$. It possibly indicates the H-(m) and H-(h) respectively. The two doublets observed at $2.83 \mathrm{ppm}$ possibly corresponds to $\mathrm{H}-\left(\mathrm{n}^{*}\right)$. Another clear doublet and doublet signals can be seen at $2.90 \mathrm{ppm}$ which indicates $\mathrm{H}$ (i). As seen in the ${ }^{1} \mathrm{H}$ NMR spectrum of free sulfo-LC-NHS-biotin, the signals correspond to H-(a) and H-(d) were seen at 3.11 ppm and 3.20 ppm respectively. Also two signals were seen at $4.28 \mathrm{ppm}$ and $4.46 \mathrm{ppm}$ which possibly correspond to $\mathrm{H}$-(c) and H-(b). The signals from H-(e)-(g), H-(j)-(l), and H-(o) and (p) were overlapped with the signals from the surfactant. However, the important point of this result is that there is no signal was observed at around 2.5 ppm which can be observed as $\mathrm{H}-(\mathrm{m})$ in free sulfo-LC-NHS-biotin. The result implies the success of the biotinylation of the silicon quantum dots capped with allylamine. To our best knowledge, there has been no report of attaching bio molecules on the surface of the silicon quantum dots therefore this result showed great progress on the biological applications of silicon quantum dots.

\section{Experimental}

$0.0012 \mathrm{~g}$ of sulfo-LC-NHS-biotin was dissolved in dimethyl sulfoxide (DMSO) and slowly added to a solution of silicon quantum dots capped with allylamine from experiment 8 from chapter 3 on ice water bath. After stirring in the dark for 4 hours unreacted free sulfo-LC-NHS-biotin was removed from size exclusion column chromatography (LH20).

\section{4-2. Optical properties of silicon quantum dots}

The optical properties of silicon quantum dots are important for their biological applications. Bulk silicon is an indirect band gap semiconductor material, therefore when electrons come back into the valence band after they were excited to the conduction band the energy will be released mainly as vibration. However, it is known that when crystalline silicon becomes nano scale with a core size smaller than 
its exciton Bohr radius it starts to emit fluorescence. The absorption spectra and the photoluminescence spectra of silicon quantum dots with different terminal functional groups of the surface moieties were measured and will be discussed in the following section. The excitation wavelengths were chosen considering the results of absorption spectra (Figure 4-19).

\section{4-2-1: Absorption spectra}

The absorption spectra were taken on an Agilent UV-Visible spectrophotometer for each silicon quantum dot sample. 1,5-hexadiene capped silicon quantum dots were measured in hexane and epoxide terminated silicon quantum dots were measured in dichloromethane. Both diol terminated and amine terminated silicon quantum dots were measured in methanol. Absorption was measured between $210 \mathrm{~nm}$ and $450 \mathrm{~nm}$. The results of each sample were shown in Figure 4-19.

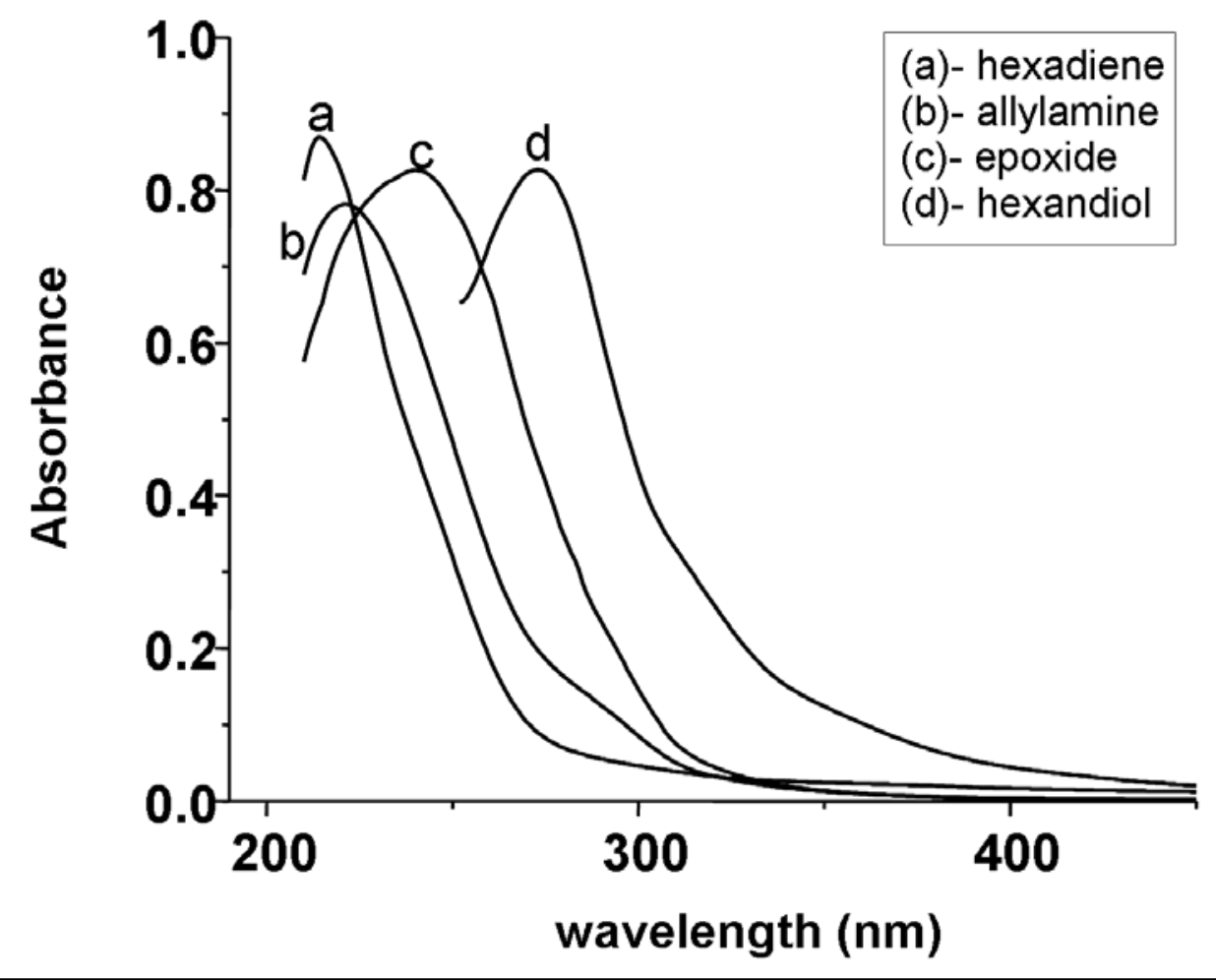

Figure 4-19: Absorption spectra of silicon quantum dots (a); capped with 1,5hexadiene, (b); capped with allylamine, (c); epoxide terminated, (d); diol terminated 
As may be seen in Figure 4-19, silicon quantum dots capped 1,5-hexadiene showed a narrow absorption peak at $220 \mathrm{~nm}$ region. Compared to the other silicon quantum dots the absorption peak of the silicon quantum dots capped with 1,5-hexadiene is blue shifted around 10 to $80 \mathrm{~nm}$. Since the nanoparticles of the silicon quantum dots capped with 1,5-hexadiene, epoxide terminated silicon quantum dots and silicon quantum dots capped with hexandiol are identical the difference of the absorption purely depends on the functional groups on the terminal ends.

\section{4-2-2: Photoluminescence spectra}

The photoluminescence spectra of silicon quantum dots capped with 1,5-hexadiene, epoxide terminated silicon quantum dots, diol terminated silicon quantum dots and amine terminated silicon quantum dots were measured by a HORIBA Jobin Yvon TCSPC Fluorolog spectrophotometer. Each sample was taken in the same solvent as used for photoluminescence spectra. The photoluminescence of 1,5-hexadiene capped silicon quantum dots were measured using excitation wavelengths from $260 \mathrm{~nm}$ to $400 \mathrm{~nm}$ with $20 \mathrm{~nm}$ intervals. Epoxide and diol terminated silicon quantum dots were excited from $320 \mathrm{~nm}$ to $420 \mathrm{~nm}$ with $20 \mathrm{~nm}$ intervals. For allylamine capped silicon quantum dots, the sample was excited from $340 \mathrm{~nm}$ to $420 \mathrm{~nm}$ with $20 \mathrm{~nm}$ intervals. The results are shown in Figure 4-20.

These excitation wavelengths were chosen based on their absorption spctra. 


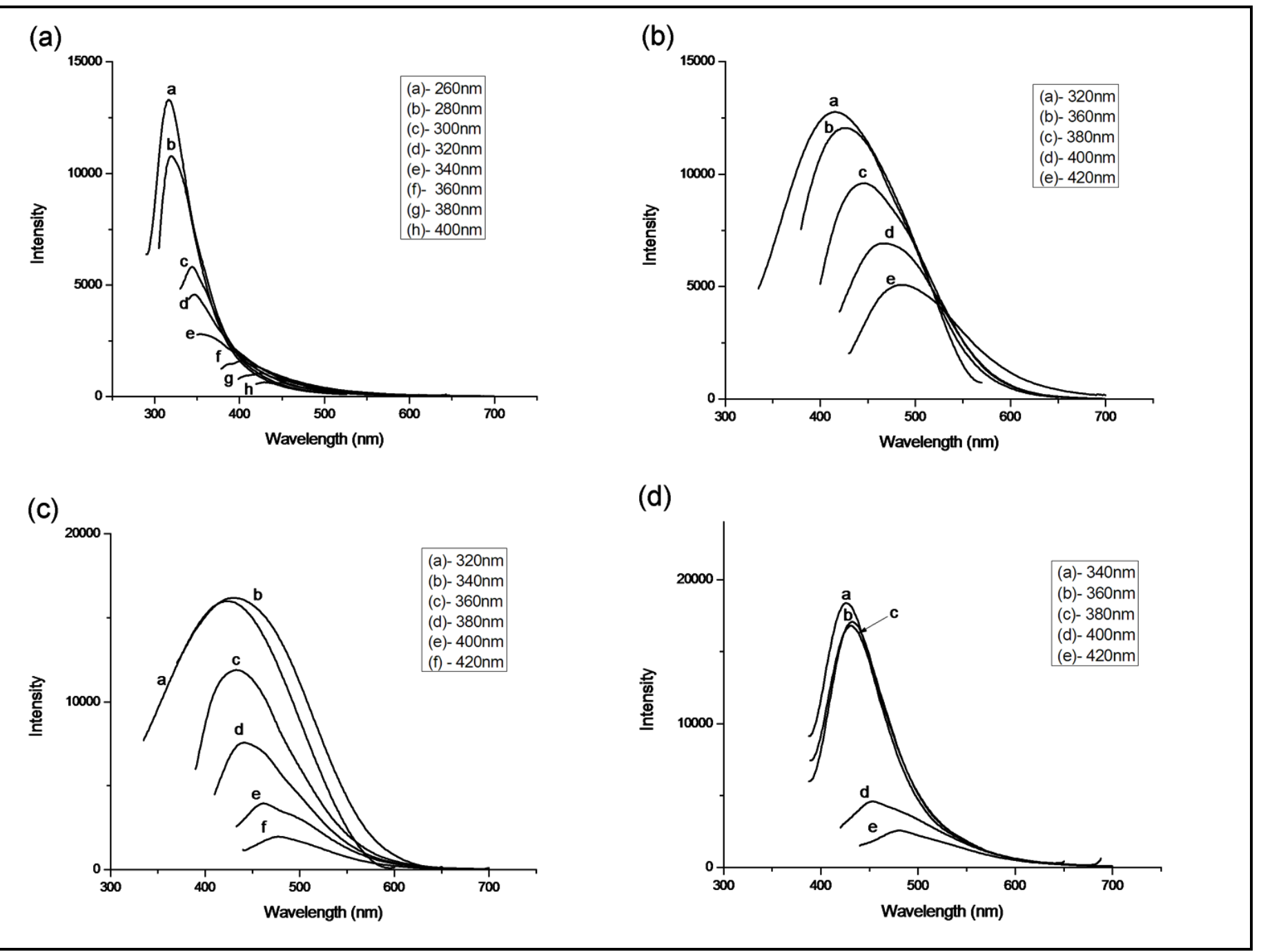

Figure 4-20: Photoluminescence spectra of silicon quantum dots (a); capped with 1,5-hexadiene, (b); epoxide terminated, (c); diol terminated 
Silicon quantum dots capped with 1,5-hexadiene showed a maximum peak of photoluminescence at around $330 \mathrm{~nm}$ when they were excited at $260 \mathrm{~nm}$. For epoxide terminated silicon quantum dot, the maximum peak was at $410 \mathrm{~nm}$ when it was excited at $320 \mathrm{~nm}$. For diol terminated and amine terminated silicon quantum dots, the maximum peaks were at around $430 \mathrm{~nm}$ and $420 \mathrm{~nm}$ when they were excited at 320 $\mathrm{nm}$ and $340 \mathrm{~nm}$ respectively. In all the spectra the peaks of photoluminescence shifted to higher wavelengths according to higher excitation wavelength and the intensity of the photoluminescence became lower.

The origin of the photoluminescence in silicon quantum dots is complicated by the presence of both indirect and direct band gap transitions. The blue luminescence has been observed for silicon quantum dots and can be attributed to the $\Gamma-\Gamma$ direct band gap transition and is in good agreement with previous reports. ${ }^{16-19}$

The observation that silicon quantum dots capped with non-polar diene functionalities have a higher energy emission than quantum dots terminated with polar epoxy, diol and amine functional groups is of interest, and similar to previous observations comparing the optical properties of silicon quantum dots capped with non-polar and polar molecules. ${ }^{19}$ As mentioned in optical absorption section the nanocrystal sizes of 1,5-hexadiene capped and hexandiol terminated silicon quantum dots are almost identical from TEM images and therefore the origin of the difference in the energy of the emission is most likely due to the nature of the surface capping and environment. ${ }^{15}$ This result provides direct evidence that the surface-capping molecule plays an important role in the radiative recombination mechanisms in 2-4 nm silicon nanocrystals. The difference in the emission is most likely due to different radiative recombination pathways for polar and non-polar capped silicon nanocrystals. ${ }^{15}$ Theoretic studies have shown that it possible for the electronic charge distribution of polar-capped silicon nanocrystals to be modified by the polar nature of the capping ligand leading to the band gap being slightly lower for polar capped silicon nanocrystals than for non-polar capped silicon nanocrystals. ${ }^{20-22}$ This may be occurring in the samples studied here. 


\section{4-3. Cyto-toxicity evaluation of silicon quantum dots}

The potential use of quantum dots in biological applications necessitates toxicity evaluations of these materials. For biological applications, quantum dots of group II/VI systems such as CdSe quantum dots are well-known, and substantial research has been conducted on these systems because of their strong luminescence. ${ }^{23}$ There have, however, been concerns over the toxicity of these quantum dots in the human body. The toxicity of these quantum dots arises from two main sources: (1) the quantum dot core and (2) the capping molecules. The cyto-toxicity of CdSe quantum dots has been reported by Derfus et al. ${ }^{24}$ who illustrated that cell damage could be caused by an uncoated CdSe core under UV excitation. Because bulk silicon has the advantage of being nontoxic and inexpensive, silicon nanoparticles would be an ideal candidate for biological fluorescence imaging. The cyto-toxicity of alkyl-capped silicon quantum dots was reported by Datta et. al. ${ }^{25}$ According to their study there is a marked difference in the rate and extent of intracellular accumulation of the alkylcapped silicon quantum dots between human cancerous and non-cancerous primary cells. The malignant cells showed higher rate and extent compared to normal human primary cells. In this section the cell viability assay (MTT assay) of silicon quantum dots with different surface molecules were undertaken to evaluate any difference of cyto-toxicity resulting from the surface moieties. The MTT assay is a colourimetoric assay for measuring the activity of enzymes in mitochondria called mitochondrial reductase which reduce 3-(4,5-dimethylthiazol-2-yl)-2,5-diphenyltetrazolium bromide (MTT) to formazan in living cells. This substance has an absorbance maximum at 460 $\mathrm{nm}$. Therefore by measuring absorbance of this substance the mitochondrial activity can be measured. ${ }^{5,6}$ Epoxide, diol and amine terminated silicon quantum dots were used for this experiment. Hydrophobic silicon quantum dots such as those capped with 1,5-hexadiene could not be used because of their low solubility under biological conditions.

For the cell types, human skin fibroblasts (WS1) and epithelial cells (A549) were chosen because the skin and lungs have higher possibilities of being exposed through different exposure mechanisms. 


\section{Experimental}

Human skin fibroblasts (WS1), epithelial cells (A549), and hepatoma cell line (HepG2) were used. WS1 and A549 were tested with epoxide- and diol-terminated silicon quantum dots, and HepG2 was used for amine-terminated silicon quantum dots. 96-well plates were used for this experiment and 10000 cells were inoculated in each well. The cells were cultured for $48 \mathrm{~h}$ at $37{ }^{\circ} \mathrm{C} / 5 \% \mathrm{CO}_{2}$. Next, each type of silicon quantum dots were added in the indicated concentrations and cocultured for $48 \mathrm{~h}$ for WS1 and A549 cells and $1 \mathrm{~h}$ for HepG2. Cell Counting Kit-8 (Dojindo, Japan) was used to measure the succinate dehydrogenase mitochondrial activity. The $450 \mathrm{~nm}$ absorption of formazans produced by the enzyme was measured with a DTX 880 (Beckman Coulter, Inc., USA) microplate reader $(n=3)$. The activities were calculated as the ratio of the absorbance value against those of the control. For control, the cell culture medium (DMEM) with $3 \%$ of ethanol (Et) was used to culture the cells since the silicon quantum dots in ethanol were added in DMEM to co-culture with the cells. 


\section{Results}

(a)

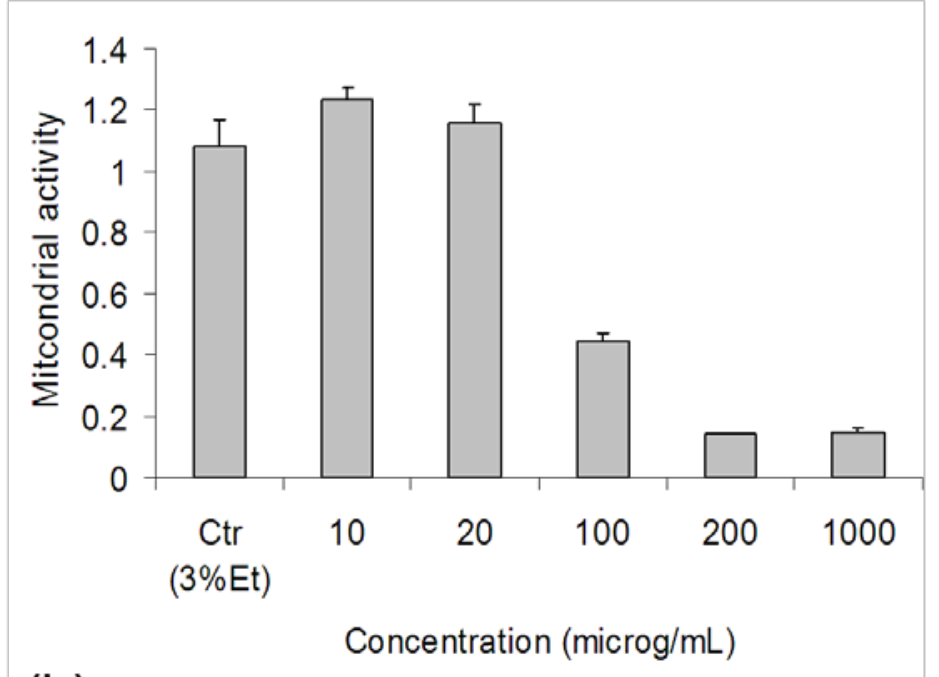

(b)

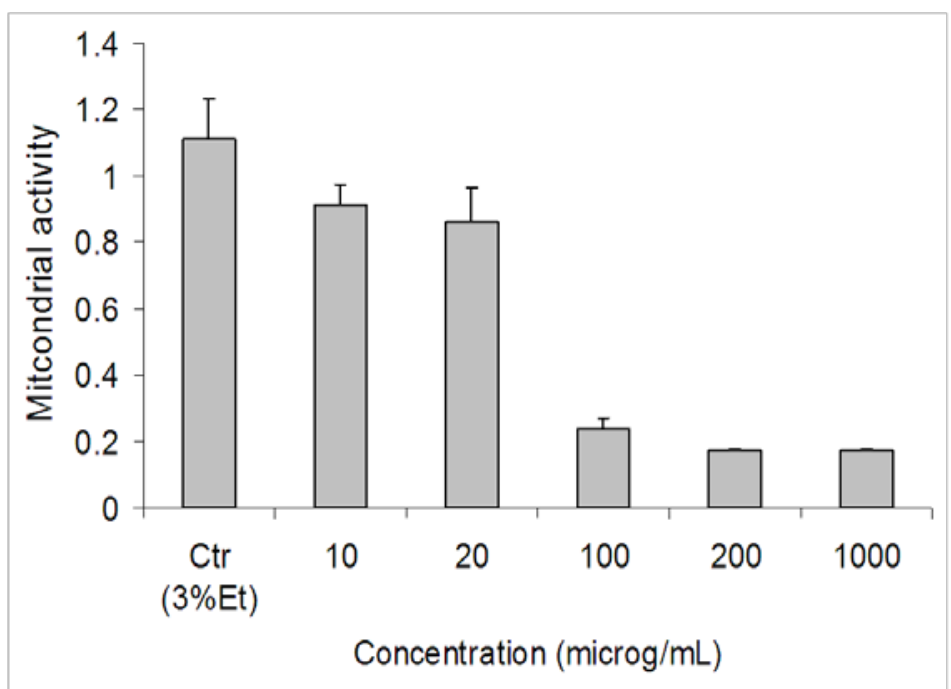

Figure 4-21: Cell viability assay on HepG2 (a); amine terminated silicon quantum dot, (b); biotin terminated silicon quantum dot 


\section{(a)}

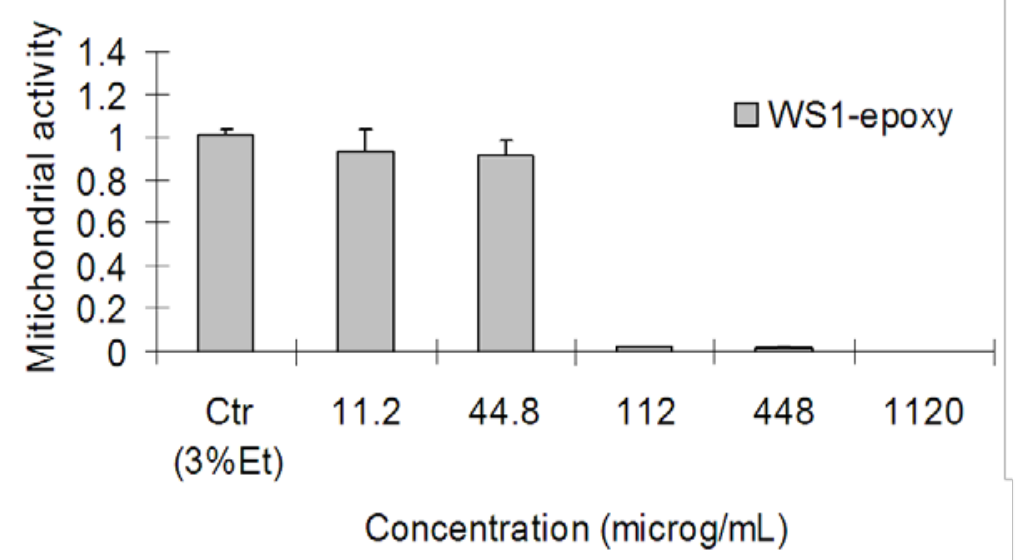

(b)

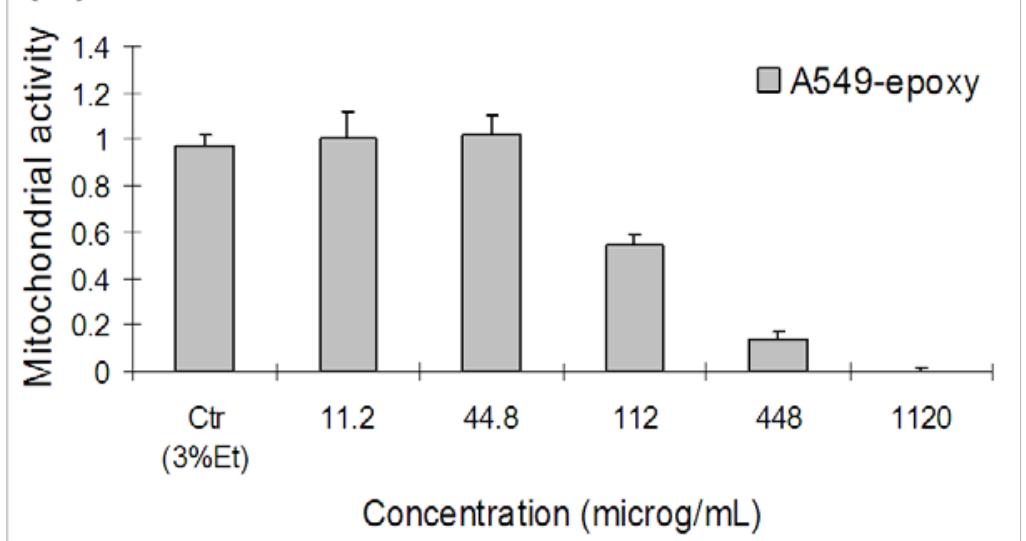

(c)

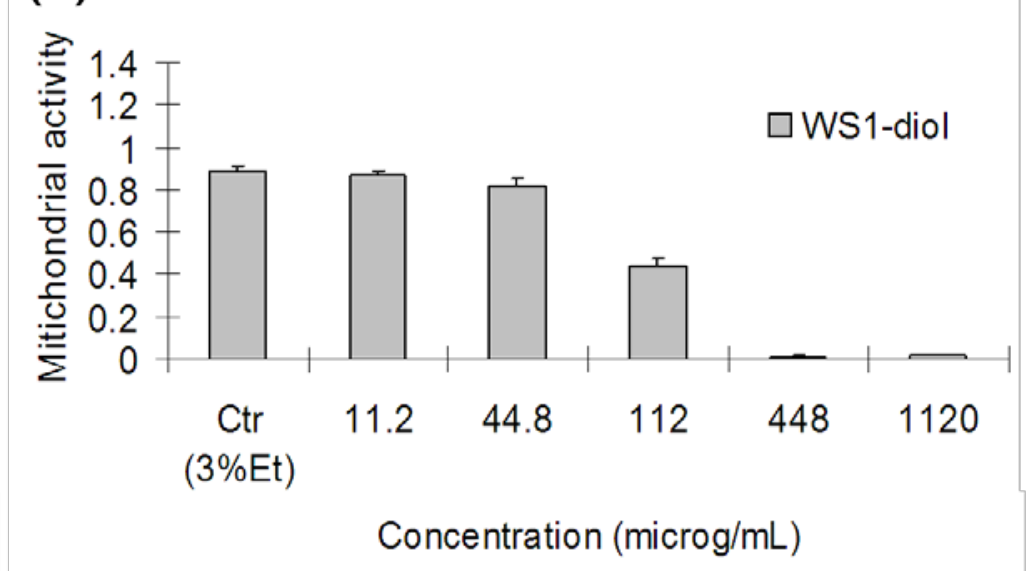

(d)

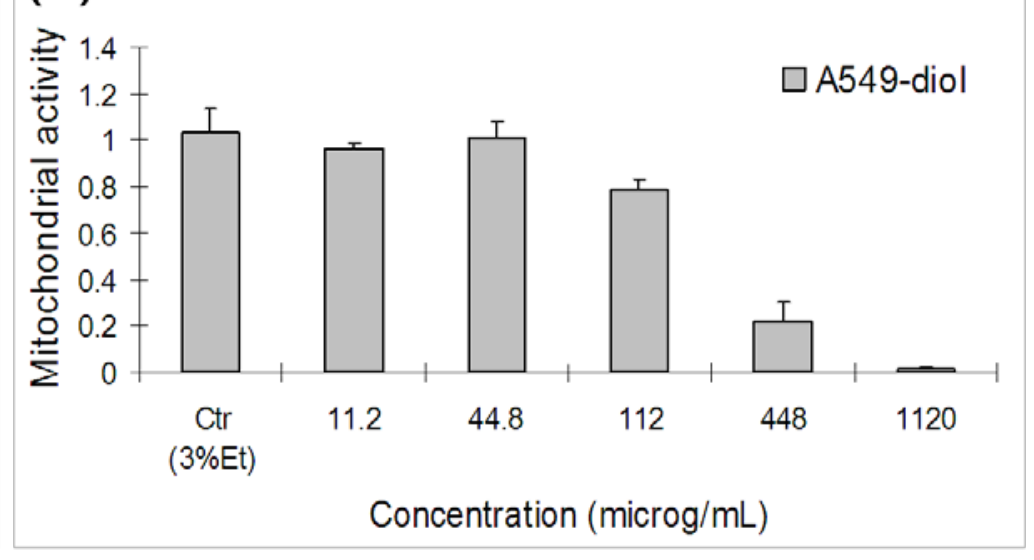

Figure 4-22: (a), (b); cell viability assay of epoxide terminated silicon quantum dot on WS1 cells and A549 cells respectively, (c), (d); cell viability assay of diol terminated silicon quantum dot on WS1 cell and A549 cells respectively 
The results of the cell viability assay were shown in Figure 4-21and Figure 4-22. As can be seen in the Figure 4-22, for A549 cells, the epoxide terminated quantum dot cyto-toxicity appears at a concentration of $112 \mu \mathrm{g} / \mathrm{mL}$. In comparison the diol terminated silicon quantum dots did not show toxicity at this level but at $448 \mu \mathrm{g} / \mathrm{mL}$. The $50 \%$ inhibition coefficients of epoxide terminated silicon quantum dot for WS1 cell and A549 cell were $0.068 \mathrm{mg} / \mathrm{mL}$ and $0.13 \mathrm{mg} / \mathrm{mL}$ respectively. In comparison, the $50 \%$ inhibition coefficients for the diol terminated silicon quantum dots did not appear until $0.097 \mathrm{mg} / \mathrm{mL}$ and $0.225 \mathrm{mg} / \mathrm{mL}$ for WS1 cell and A549 cell lines which is almost double the concentration compared to the epoxide terminated quantum dots. These figures are comparable to previous studies on quantum dots that have relatively low toxicity.

To compare the toxicity on these two cell lines, toxicity on hepatoma cell line (HepG2) was evaluated with amine terminated and biotinylated silicon quantum dots. This cell line was chosen because liver has the function to reduce toxicity by albumin secretion or glucuronate conjugation among the tissues in which quantum dots may accumulate. $^{26}$

For the amine terminated and biotinylated silicon quantum dots, the cell viability showed similar results. Both silicon quantum dots showed cyto-toxicity around 0.1 $\mathrm{mg} / \mathrm{mL}$. However, biotinylated silicon quantum dots showed slightly higher toxicity than amine terminated silicon quantum dots. The results showed 50\% inhibition coefficient of amine terminated and biotinylated silicon quantum dots in the hepatoma cell line (HepG2) was $0.1 \mathrm{mg} / \mathrm{mL}$ and $0.06 \mathrm{mg} / \mathrm{mL}$ respectively. The amine terminated silicon quantum dots showed the similar results to the diol terminated silicon quantum dots in WS1 cells.

The results imply safe uses of silicon quantum dots in biological application below the concentration of the $50 \%$ inhibition coefficients which is called the thresholds. 


\section{4-4. Bio-imaging of silicon quantum dots in cells}

Organic dyes such as fluorescein-4-isothiocyanate (FITC) and rhodamine have been widely used for bio-imaging, however long term observation has been difficult because of their poor photo stability. Quantum dots have been introduced as new candidates to overcome this problem where the cell uptake stability of the silicon quantum dots are very important. These attributes of amine terminated silicon quantum dots were evaluated with HepG2 and the fluorescence of the quantum dots was observed using fluorescent microscope.

\section{Experimental}

Silicon quantum dots capped with allylamine were applied for cell imaging. HepG2 cells were used. The assay was conducted at $1.0 \mu \mathrm{g} / \mathrm{mL}$ for $6 \mathrm{~h}$ incubation. Images were taken on a fluorescent microscope IX-81 (Olympus) with an excitation filter of 330-380 nm. The emission was monitored at $480 \mathrm{~nm}$.

\section{Results}

Figure 4-23 shows the cell imaging of silicon quantum dots capped with allylamine. This image shows the suitability of allylamine-capped silicon quantum dots as a chromophore for biological imaging. The transmitted light and fluorescent control images of the HepG2 are shown in Figure 4-23 a and b respectively. Figure 4-23 c and d show the transmitted light and fluorescent images of the HepG2 co-cultured with the silicon quantum dots. The fluorescent control image of the HepG2 (Figure 4-23 b) shows almost no fluorescence. Also no blue luminescence was observed in the transmitted light image of the control (Figure 4-23 a). Thus, the fluorescence observed in the HepG2 in Figure 4-23 c and d arises from the emission from silicon quantum dots. The inset in the bottom right corner of Figure 4-23 d shows the fluorescence from a vial of allylamine capped silicon quantum dots in water when excited with UV light. The bright blue fluorescence from the silicon quantum dots in the cells is distributed uniformly and shows that the silicon quantum dots were taken up into the cytoplasm. This result shows the possibility of using these hydrophilic silicon quantum dots as chromophores in biological fluorescence imaging. 


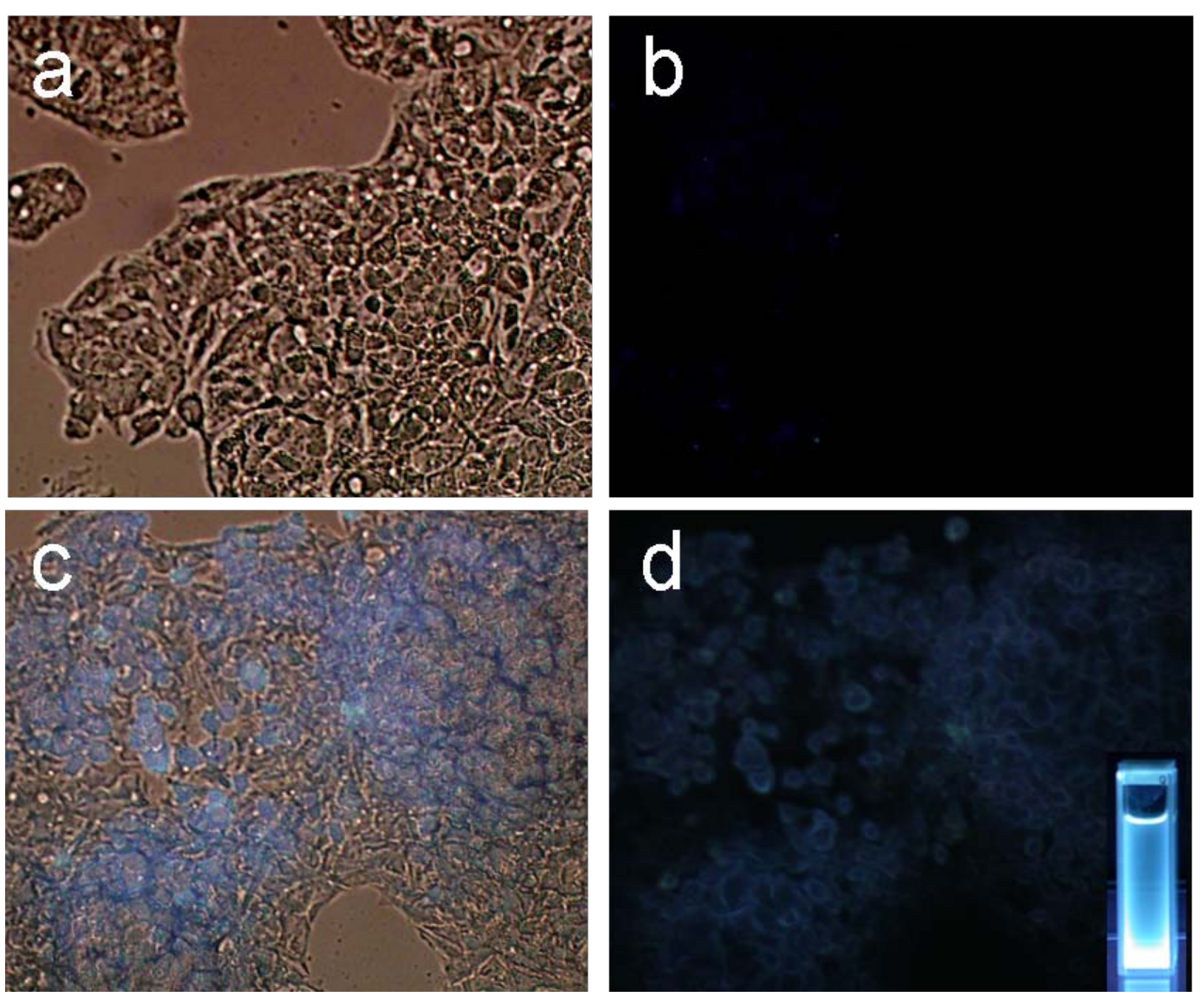

Figure 4-23: (a) and (b); transmitted light and fluorescent control images of the HepG2 respectively. (c) and (d); transmitted light and fluorescent images of the HepG2 co-cultured with the silicon quantum dots capped with allylamine. 


\section{4-5. Summary and Discussion}

Experiment 4-1-1 and 4-1-2 showed the oxidation of the terminal double bond on the surface of silicon quantum dots. From ${ }^{1} \mathrm{H}$ NMR and FTIR analysis, the features of epoxide rings and diol groups respectively could be seen very clearly. The nanoparticles observed in TEM micrographs of silicon quantum dot capped with 1,5hexadiene and silicon quantum dots obtained from experiment 4-1-2 are almost identical. Nanoparticles could not be observed from the sample of experiment 4-1-1. This is probably because high reactivity of epoxy group. ${ }^{27}$ The amine capped silicon quantum dots reacted with biotin seemed to have weaker luminescence compared to the amine terminated silicon quantum dots. Since sulfo-LC-NHS-biotin has relatively long carbon chain, the excited electrons might have been quenched. Kim et. al reported the quantum yields of the photoluminescence of the CdSe quantum dots capped with different ligands which have different chain lengths. They concluded that final quantum yield of longer-chain ligand system is less than those from short-chain ligand system. ${ }^{28}$ Also Li et. al reported photoluminescence quench of CdSe quantum dots embedded in silica shell which imprinted with the molecule called lambdacyhalothrin(LC). ${ }^{29}$ In their research the molecule is imprinted to silica shell also through amine group. They gave the possible reason of the quench as the formation of an efficient hole or electron acceptor which introduces new nonradiative decay pathways for the exciton. ${ }^{29}$

Since the particle sizes are almost identical from TEM micrograph, the results of optical properties measurement showed that optical properties of silicon quantum dots can change depending on the surface molecules. Silicon quantum dots capped with non-polar molecules showed slightly higher absorption and emission energy compared to the ones capped with polar molecules.

Frauenheim et. al have reported the effect of the surface functional groups of silicon quantum dots at the excited state on optical properties including optical absorption and emission energies. They investigated the optical properties of silicon quantum dots with the terminal groups of $\mathrm{H}, \mathrm{CH}_{3}, \mathrm{NH}_{2}, \mathrm{SH}$, and $\mathrm{OH}$ by using densityfunctional tight-binding calculations. ${ }^{30}$ According to these calculations, when the electron negativity is larger, the energy gap between the highest occupied molecular 
orbital (HOMO) and lowest unoccupied molecular orbital (LUMO) becomes smaller. This is due to geometric structure of silicon quantum dots. ${ }^{31}$ For silicon quantum dots capped with hydrophobic terminal groups the silicon atom connecting to the centre silicon atom shifts away from its original position when the quantum dot is excited from the ground state to the first excited state. This is due to excited-state relaxation and it leads to an elongated first-neighbour Si-Si bond. On the other hand, the elongated Si-Si bond in silicon quantum dots capped with hydrophilic terminal groups are the outer-layer Si-Si bonds rather than the inner Si-Si bonds. This difference could be explained by an electronegativity comparison. The electron negativity of Si (1.90) is close to those of $\mathrm{H}$ (2.10) and C (2.55), but much smaller than those of $\mathrm{N}$ (3.04) and $\mathrm{O}$ (3.44) which indicates that $\mathrm{N}$ and $\mathrm{O}$ are much stronger than $\mathrm{H}, \mathrm{C}$, and $\mathrm{Si}$ in terms of drawing electrons towards them. ${ }^{32}$ Therefore in silicon quantum dots capped with hydrophilic terminal groups, the outer-layer Si-Si bonds show a much stronger drawing effect from the surface groups than inner $\mathrm{Si}$-Si bonds, and thus are more easier to be elongated. Furthermore the nature of the electronic state responsible for absorption and emission processes is different depending on the surface functional groups. In silicon quantum dots capped with non-polar molecules, both HOMOs and LUMOs are delocalised throughout the core of the nanoparticles. In contrast, for silicon quantum dots capped with polar molecules, the HOMOs and LUMOs are drawn to the surface towards the polar functional groups. ${ }^{33}$ The results of optical properties of the silicon quantum dots investigated in this research showed a good agreement with this theory.

The results of cell viability assay showed that the 50\% inhibition coefficient of the concentration of diol terminated silicon quantum dots was twice as high as epoxide terminated ones. The epoxide group is a highly reactive species and known to have oxidative toxicity and so it is in agreement with expectations that it has a higher toxicity than the diol. ${ }^{27}$ A549 showed higher 50\% inhibition coefficients in both epoxide and diol terminated silicon quantum dots. The reason for the observation is most likely due to the difference of the metabolism of each cell line which would affect the uptake of the silicon quantum dots. It would make a difference in the amount of the silicon quantum dots taken up in the each cell line. ${ }^{34}$

Since there are various ways to assess the toxicity and diversity of cell types, to get a general idea of cyto-toxicity of quantum dots has been difficult. However MTT assay 
is one of the most common assays applied for the toxicity evaluation of CdSe quantum dots. Li et. al reported that without antioxidant molecules, CdSe quantum dot showed severe cyto-toxicity at $0.01 \mathrm{mg} / \mathrm{mL} .^{35}$ In their research, nearly $20 \%$ of the cadmium was released after 24 hours incubation as cadmium ions which cause apoptosis to the cells. Although Derfus et. al published research which can prevent the leak of cadmium ion by coating the CdSe core with $\mathrm{ZnS}$ shell and also covered with albumin, when subcellular localisation of quantum dots or their local environment changes, or they are retained in cells for a long term the degradation of coatings can occur and expose their inorganic core. ${ }^{24,36,37}$ Compared to the oxidative stress of the CdSe or CdTe quantum dots, silicon quantum dots have much less potential to cause apoptosis even when the core part is exposed. When the CdSe quantum dots are oxidised, the selenium of the core form an oxide compound and releases cadmium ions. On the other hand, when silicon quantum dots are oxidised, they form a silica layer on the surface. Silica is also known to be non-toxic to human and there have been a few reports of cyto-toxicity of silica nanoparticles which showed much higher thresholds compared to CdSe. ${ }^{38}$ 


\section{4-6. References}

1. http://home.hiroshima-u.ac.jp/takedake/G94.pdf

2. Z. F. Li, E. Ruckenstein; Nano Lett., 2004, 4, 1463-1467, "Water-Soluble Poly(acrylic acid) Grafted Luminescent Silicon Nanoparticles and Their Use as Fluorescent Biological Staining Labels

3. S. Sato, M. T. Swihart; Chem. Mater., 2006, 18, 4083-4088, "Propionic-AcidTerminated Silicon Nanoparticles: Synthesis and Optical Characterization”

4. C. Tu, X. Ma, P. Pantazis, S. M. Kauzlarich, and A. Y. Louie; J. Am. Chem. Soc., 2010, 132, 2016-2023, "Paramagnetic, Silicon Quantum Dots for Magnetic Resonance and Two-Photon Imaging of Macrophages”

5. http://en.wikipedia.org/wiki/MTT_assay

6. http://chokai.riast.osakafu-u.ac.jp/ housya2/shiizaki/MTT.html

7. R. M. Silverstein, and F. X. Webster; Spectrometric Identification of Organic Compounds, John Wiley \& Sons, Inc., New York, United States of America, 1996.

8. R. D. Tilley, J. H. Warner, K. Yamamoto, I. Matsui, and H. Fujimori; Chem. Commun., 2005, 14, 1833-1835, "Micro-emulsion synthesis of mono disperse surface stabilized silicon nanocrystals”

9. R. S. Iyer, and T. M. Harris; Chem. Res. Toxicol., 1993, 6, 313-316, "Preparation of Aflatoxin B 8,9-Epoxide Using m-Chloroperbenzoic Acid"

10. R. H. Fan, and X. L. Hou; Org. Biomol. Chem., 2003, 1, 1565-1567, “Tetrabutylammonium bisulfate: a new effective catalyst for the hydrolysis of aziridines or epoxides” 
11. Q. S. Li, R. Q. Zhang, and S. T. Lee; Appl. Phys. Lett., 2007, 91, 043106, "Stabilizing excited-state silicon nanoparticle by surface oxidation”

12. http://www.organic-chemistry.org/namedreactions/jones-oxidation.shtm

13. http://en.wikipedia.org/wiki/Biotin

14. A. Baeza, I. I. Barba, and M. V. Regi; Acta Biomater., 2010, 6, 743-749, "Biotinylation of silicon-doped hydroxyapatite: A new approach to protein fixation for bone tissue regeneration"

15. S. R. S. Ting, T. L. U. Nguyen, and M. H. Stenzel; Macromol. Biosci., 2009, 9, 211-220, “One Pot Synthesis of Surface PEGylated Core-Shell Microparticles by Suspension Polymerization with Surface Enrichment of Biotin/Avidin Conjugation”

16. J. D. Holmes, K. J. Ziegler, R. C. Doty, L. E. Pell, K. P. Johnston, and B. A. Korgel; J. Am. Chem. Soc., 2001, 123, 3743-3748, "Highly Luminescent Silicon Nanocrystals with Discrete Optical Transitions”

17. J. P. Wilcoxon, G. A. Samara, and P. N. Provencio; Phys. Rev. B, 1999, 60, 2704-2714, “Optical and electronic properties of Si nanoclusters synthesized in inverse micelles”

18. J. H. Warner, A. Hoshino, K. Yamamoto, and R. D. Tilley; Angew. Chem. Int. Ed., 2005, 44, 4550-4554, “Water-Soluble Photoluminescent Silicon Quantum Dots”

19. J. H. Warner, H. R. Dunlop, and R. D. Tilley; J. Phys. Chem. B, 2005, 109, 19064-19067, “Surface Morphology Dependent Photoluminescence from Colloidal Silicon Nanocrystals"

20. F. A. Reboredo, and G. Galli; J. Phys. Chem. B, 2005, 109, 1072-1078, “Theory of Alkyl-Terminated Silicon Quantum Dots" 
21. B. G. Walker, S. C. Hendy, R. Gebauer, and R. D. Tilley; Eur. Phys. J. B, 2008, 66, 7-15, “Application of Lanczos-based time-dependent densityfunctional theory approach to semiconductor nanoparticle quantum dots”

22. Z. Zhou, L. Brus, and R. Friesner; Nano Lett., 2003, 3, 163-167, "Electronic Structure and Luminescence of 1.1- and 1.4-nm Silicon Nanocrystals: Oxide Shell versus Hydrogen Passivation”

23. M. Nirmal, B. O. Dabbousi, M. G. Bawendi, J. J. Macklin, J. K. Trautman, T. D. Harris, and L. E. Brus; Nature, 1996, 383, 802-804, "Fluorescence intermittency in single cadmium selenide nanocrystals”

24. A. M. Derfus, W. C. W. Chan, and S. N. Bhatia; Nano Lett., 2004, 4, 11-18, "Probing the Cytotoxicity of Semiconductor Quantum Dots"

25. N. H. Alsharif, C. E. M. Berger, S. S. Varanasi, Y. Chao, B. R. Horrocks, and H. K. Datta; Small, 2009, 5, 221-228, “Alkyl-Capped Silicon Nanocrystals Lack Cytotoxicity and have Enhanced Intracellular Accumulation in Malignant Cells via Cholesterol-Dependent Endocytosis”

26. H. Honda, T. Nojima, and T. Kobayashi; Biotechnol. Lett., 1995, 17, 365-370, "Effect of additives such as anti-oxidative agent on albumin secretion from primary culture of rat hepatocyte”

27. A. Hemminki, T. Väyrynen, and K. Hemminki; Chem.-Biol. Interact, 1994, 93, 51-58, "Reaction kinetics of alkyl epoxides with DNA and other nucleophiles"

28. W. Kim, S. J. Lim, S. J.ung, and S. K. Shin; J. Phys. Chem. C, 2010, 114, 1539-1546, "Binary Amine-Phosphine Passivation of Surface Traps on CdSe Nanocrystals"

29. H. Li, Y. Li, and J. Cheng; Chem. Mater. 2010, 22, 2451-2457, "Molecular Imprinted Silica Nanospheres Embedded CdSe Quantum Dots for Highly 
Selective and Sensitive Optosensing of Pyrethroids”

30. Q. S. Li,1 R. Q. Zhang,1, S. T. Lee, T. A. Niehaus, and Th. Frauenheimz; J. Chem. Phys, 2008, 128, 244714, “Optimal surface functionalization of silicon quantum dots"

31. See EPAPS Document No. E-JCPSA6-129-606825 for the geometric parameters of the optimized structures of $\mathrm{Si}_{35} \mathrm{H}_{36}, \mathrm{Si}_{35}\left(\mathrm{CH}_{3}\right)_{36}, \mathrm{Si}_{35}(\mathrm{SH})_{36}$, $\mathrm{Si}_{35}\left(\mathrm{NH}_{2}\right)_{36}$, and $\mathrm{Si}_{35}(\mathrm{OH})_{36}$ in the ground and first excited states. For more information on EPAPS, see http://www.aip.org/pubservs/epaps.html

32. The Nature of the Chemical Bond and the Structure of Molecules and Crystals, edited by L. Pauling; Cornell Univeristy Press, NY, 1960, pp. 88-91

33. A. Puzder, A. J. Williamson, J. C. Grossman, and G. Galli; J. Am. Chem. Sco., 2003, 125, 2786-2791, "Computational Studies of the Optical Emission of Silicon Nanocrystals”

34. A. Shiohara, A. Hoshino, K. Hanaki, K. Suzuki, and K. Yamamoto; Microbiol. Immunol. 2004, 48, 669-675, “ On the Cyto-Toxicity Caused by Quantum Dots"

35. K. G. Li, J. T. Chen, S. S. Bai, X. Wena, S. Y. Song, Q. Yua, J. Li, Y. Q. Wanga; Toxicol. Invtro, 2009, 23, 1007-1013, “Intracellular oxidative stress and cadmium ions release induce cytotoxicity of unmodified cadmium sulfide quantum dots"

36. X. H. Gao, Y. Y. Cui, R. M. Levenson, L. W. K. Chung, S. M. Nie; Nature Biotechnol., 2004, 22, 969-976, “In vivo cancer targeting and imaging with semiconductor quantum dots”

37. J. Lovrić, S. J. Cho, F. M. Winnik, and D. Maysinger; Chem. Biol., 2005, 12, 1227-1234, “Unmodified Cadmium Telluride Quantum Dots Induce Reactive Oxygen Species Formation Leading to Multiple Organelle Damage and Cell 


\section{Death”}

38. M. Fisichella, H. Dabboue, S. Bhattacharyya, M. L. Saboungi, J. P. Salvetat, T. Hevor, and M. Guerin; Toxicol. Invtro, 2009, 23, 697-703, “Mesoporous silica nanoparticles enhance MTT formazan exocytosis in HeLa cells and astrocytes” 


\section{Chapter 5 Microemulsion and high temperature bench top syntheses of germanium quantum dots}

In this chapter two different systems, (microemulsion method and high temperature bench top method) were explored to produce germanium quantum dots.

There have been a few reports published about syntheses of germanium quantum dots. ${ }^{1-9}$ Miguez et. al. reported the reduction of germanium dioxide by hydrogen gas at $470{ }^{\circ} \mathrm{C} .{ }^{10}$ Heath et. al. published the reduction of halogenated germanium precursor by sodium dispersion in heptane. ${ }^{11,12}$ Also the reduction of halogenated germanium by hydride reducing agents was reported by Wilcoxon et. al. ${ }^{13}$ Each system has some advantages and drawbacks for particle size control or limited options for capping agents.

Like silicon, germanium is also expected to fluoresce under its exciton Bohr radius (24.3 nm), however synthesis of well size-controlled germanium quantum dots which give off luminescence has proven difficult. Tilley and co-workers have published well size-controlled water soluble germanium quantum dots synthesized by the microemulsion method. ${ }^{14,15}$ Schaak et. al. also reported blue luminescent germanium nanoparticles by reducing $\mathrm{GeCl}_{4}$ by $\mathrm{NaBH}_{4}$ at room temperature. ${ }^{16}$ The germanium nanoparticles were stabilised at the surface by polyvinyl-pyrrolidone (PVP).

The attempted syntheses of the water soluble germanium quantum dots capped with allylamine by a microemulsion system were performed in the first half of this chapter. This was followed by the hydrophobic capping, with 1,5-hexadiene, with the aim of carrying out further chemical reactions on the surface for biological applications.

The other half of the chapter describes a high temperature bench top synthesis using triphenylchlorogermane as a germanium precursor which is much less volatile compared to germanium tetrachloride. The advantage of this system is that no surfactant is required which makes the purification process simpler. There are a few reports of the synthesis of well mono dispersed germanium nanoparticles by thermal 
decomposition of organogermane precursor in high boiling point solvents with the reaction temperature of 360 to $429{ }^{\circ} \mathrm{C} .{ }^{17-19}$

For the high temperature bench top synthesis, sulfur is used to promote the nucleation of the germanium nanoparticles. ${ }^{20}$ Sulfur is also less toxic than other reducing agents. The reaction was conducted in a three neck flask as described below. The precursor is heated up to 280 to $300{ }^{\circ} \mathrm{C}$ and the sulfur (dispersed in TOA by heating) was injected to form germanium quantum dots. Capping molecules were then injected to prevent the quantum dots from being oxidised. Figure 5-1 describes a three neck flask system used for the high temperature bench top synthesis. A variety of different capping agents were explored on the purpose of biological applications. TEM technique was applied to characterise the samples and FTIR analysis was applied to characterise the surface molecules.

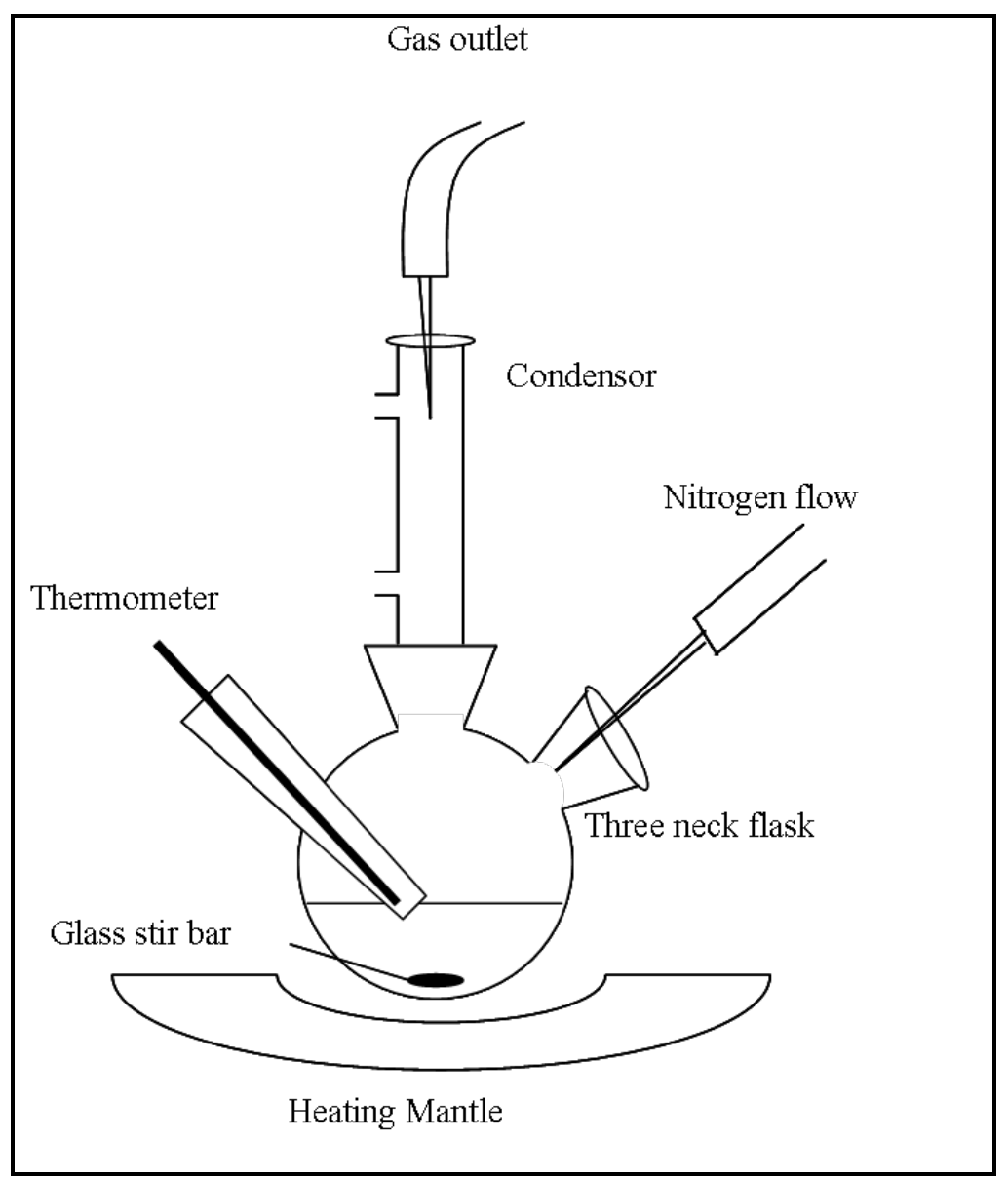

Figure 5-1: A three neck flask system used for high temperature bench top synthesis to produce germanium quantum dots. 


\section{5-1. Microemulsion synthesis of germanium quantum dots}

Following the syntheses of the silicon quantum dots, the microemulsion synthesis of germanium quantum dots was investigated for the purpose of biological applications. Germanium belongs to group IV which is the same as silicon therefore it is expected to have similar reactions as silicon. Since the germanium atom is slightly heaver compared to silicon atom and it has a larger exciton Bohr radius compared to silicon, therefore fluorescent germanium quantum dots were expected to be observed with better contrast on TEM. All experiments were conducted under nitrogen atmosphere to prevent germanium oxidation. Germanium tetrachloride was used as a precursor and formed reverse micelles with the surfacetant such as TOAB or $\mathrm{C}_{12} \mathrm{E}_{5}$ in the oragnic solvent. The precursor is then reduced to form hydrogen terminated germanium quantum dot by hydride reducing agents such as lithium alminiumhydride or lithium borohydride. The surface of this hydrogen terminated germanium quantum dot is passivated with alkenes such as allylamine or 1,5-hexadiene to prevent from being oxidised. The reaction shceme is shown in scheme 5-1-1.

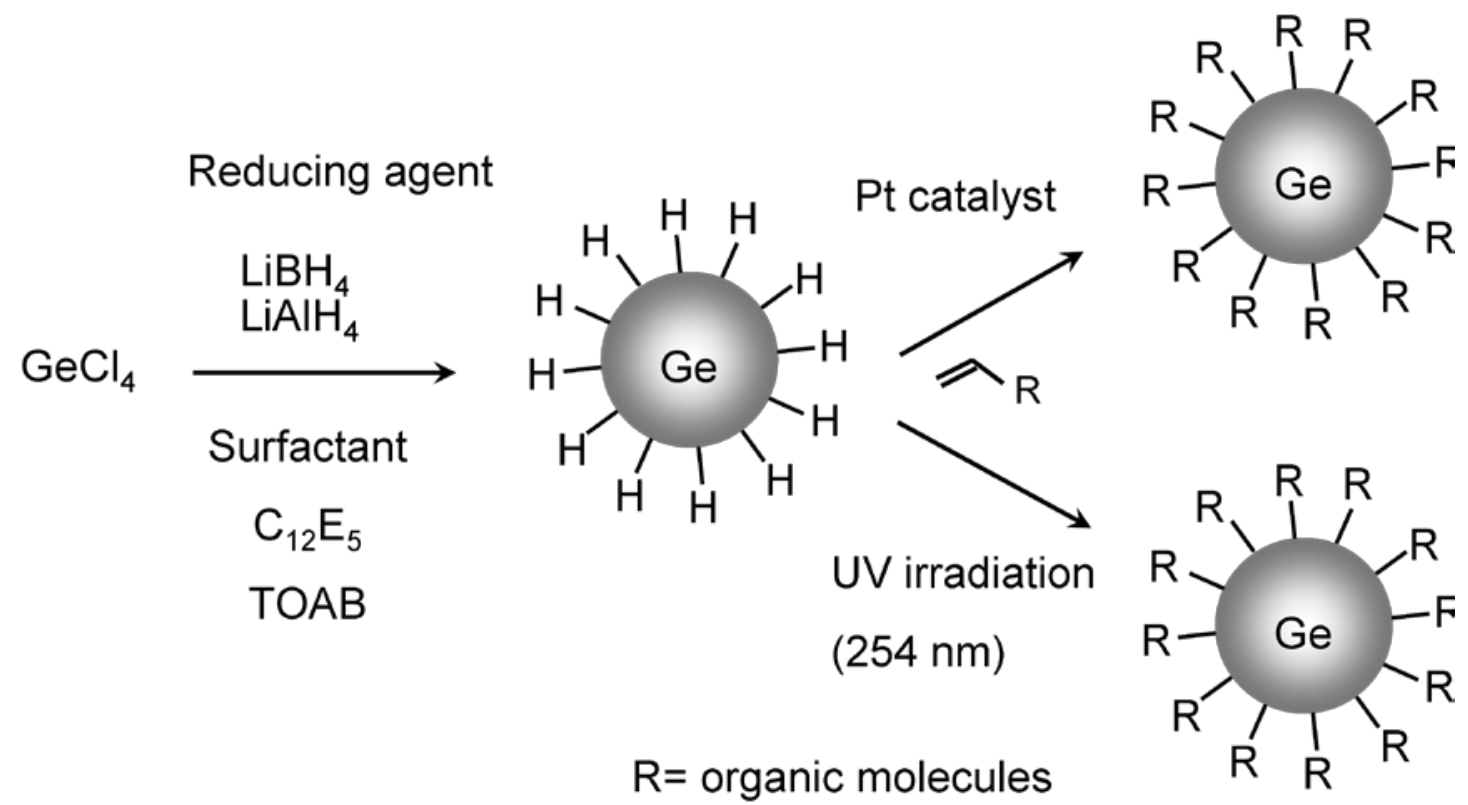

Scheme 5-1-1: Reaction scheme of a microemulsion synthesis of the germanium qunautm dot 


\section{5-1-1. Syntheses of germanium quantum dots capped with allylamine}

\section{Experiment 1}

The synthesis of germanium quantum dots capped with allylamine was conducted to produce hydrophilic quantum dots. As with silicon quantum dots the terminal double bond of allylamine was expected to form a covalent bond with germanium. An amine terminated surface would open up possibility of attaching bio-friendly molecules onto the quantum dots by reacting the terminal amine group with other functional groups such as succinimide. The sample was characterized by TEM.

\section{Experimental}

$1.4 \mathrm{~g}$ of tetraoctyl ammoniumbromide (TOAB) was dissolved in $50 \mathrm{~mL}$ of anhydrous toluene followed by the addition of $0.2 \mathrm{~mL}(0.002 \mathrm{~mol})$ of germanium tetrachloride $\left(\mathrm{GeCl}_{4}\right)$. The solution was stirred for an hour to form micelles. $4 \mathrm{~mL}$ of lithium aluminium hydride $1.0 \mathrm{M}$ as a reducing agent were injected into the solution to form hydrogen terminated germanium quantum dots. The solution turned cloudy. Also a yellow precipitate appeared in the bottom. The solution was stirred another hour and the solution became yellow with the dispersion of the precipitate. $10 \mathrm{~mL}$ of anhydrous methanol were then added drop wise to quench the reducing agent. Afterwards 0.1 $\mathrm{mL}$ of platinum norbornene as a catalyst and $2.3 \mathrm{~mL}$ of allylamine were added to the solution. The reaction mixture was stirred over night at $50{ }^{\circ} \mathrm{C}$. After the reaction, an orange precipitate was extracted by centrifugation from the reaction mixture (at 14000rpm for 20 minutes). The precipitate was washed three times with toluene to remove tetraoctyl ammoniumbromide (TOAB).

\section{Results}

The orange solid was studied under HRTEM. As seen in Figure 5-2, no spherical nanoparticles were observed, however from EDS analysis around 160 counts of germanium $\mathrm{L}_{\alpha}$ ray were observed and the peaks from aluminium or platinum were hardly observed. This indicates the product seen in the TEM micrograph contains 
germanium. The powder did not show the evidence of crystalline germanium in XRD analysis therefore the aggregates observed in the Figure 5-2 are possibly amorphous germanium. The result indicates the possibility that crystalline germanium quantum dots formed during the reaction remained in the liquid as a colloidal suspension and did not precipitate. In the next experiment, the reaction was conducted with the same capping molecule to form a hydrophilic surface and the supernatant of the sample was investigated.

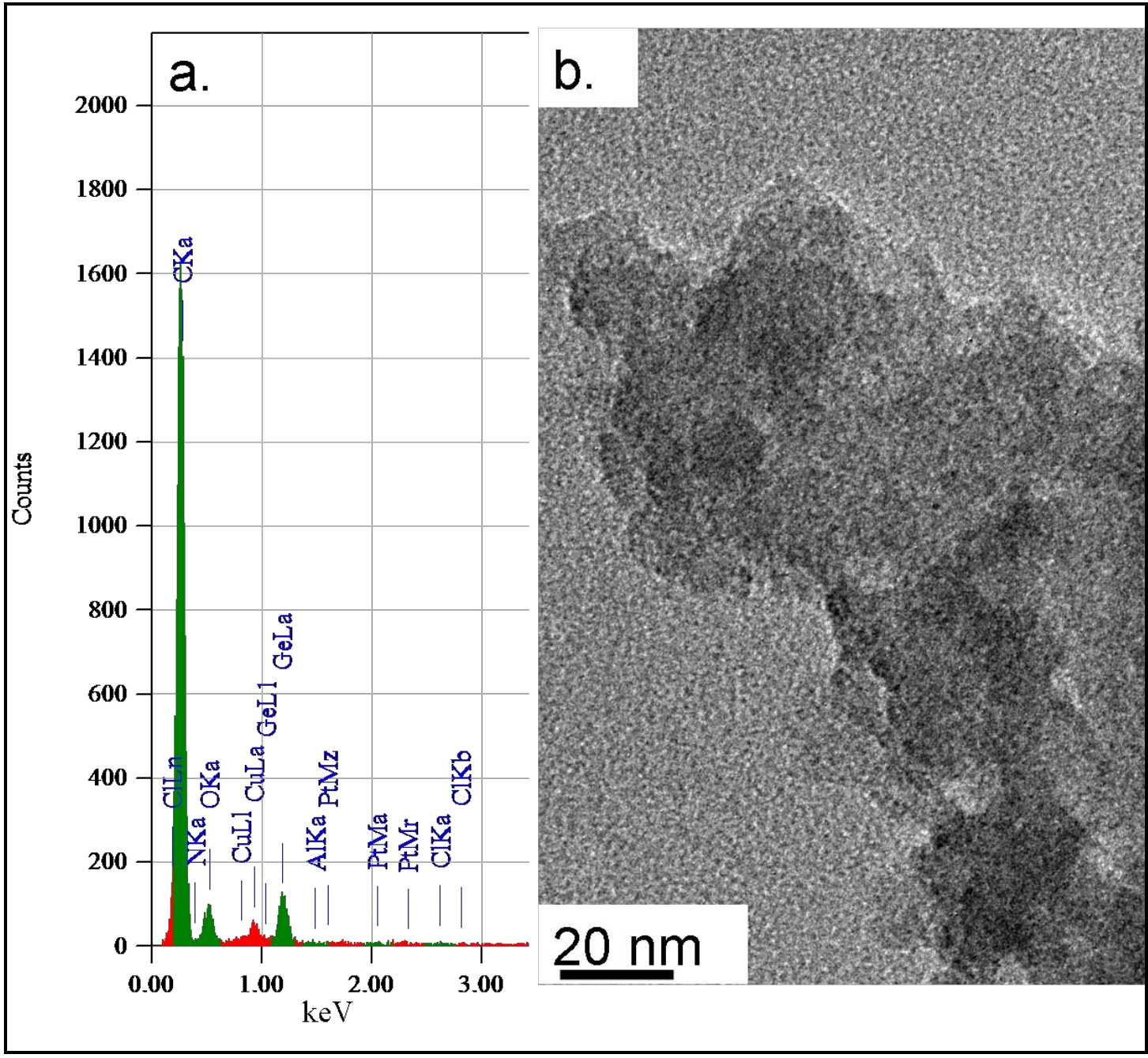

Figure 5-2: (a) EDS spectrum of the sample obtained from experiment 1, (b) a TEM micrograph of a germanium chunk obtained from experiment 1

\section{Experiment 2}

In this experiment pentaethylene glycol monododecyl ether $\left(\mathrm{C}_{12} \mathrm{E}_{5}\right)$ was used as a surfactant. Since $\mathrm{C}_{12} \mathrm{E}_{5}$ is a non-ionic surfactant the solubility in water is less than tetraoctyl ammoniumbromide which makes easier to remove from germanium 
quantum dots with a hydrophilic surface. Also ultra-violet irradiation was applied on the surface modification. This method can remove the risk of producing platinum nanoparticles from the platinum catalyst.

\section{Experimental}

$0.04 \mathrm{~g}$ of pentaethylene glycol monododecyl ether $\left(\mathrm{C}_{12} \mathrm{E}_{5}\right)$ was dissolved in $50 \mathrm{~mL}$ of anhydrous hexane followed by the addition of $0.2 \mathrm{~mL}(0.002 \mathrm{~mol})$ of germanium tetrachloride $\left(\mathrm{GeCl}_{4}\right)$. The solution was sonicated for 30 minutes to form micelles. 2.0 $\mathrm{mL}$ of lithium borohydride $2.0 \mathrm{M}$ as a reducing agent were injected into the solution to form hydrogen terminated germanium quantum dots. The solution turned cloudy. Also a yellow precipitate appeared in the bottom. The solution was stirred for another hour and the solution became yellow with the dispersion of the precipitate. $5.0 \mathrm{~mL}$ of allylamine were added to the solution. The supernatant was transferred to a UV reactor vessel and exposed to ultra-violet light $(254 \mathrm{~nm})$ for 4 hours without stirring. After the reaction the solution was concentrated and purified by liquid-phase separation with water and hexane.

\section{Results}

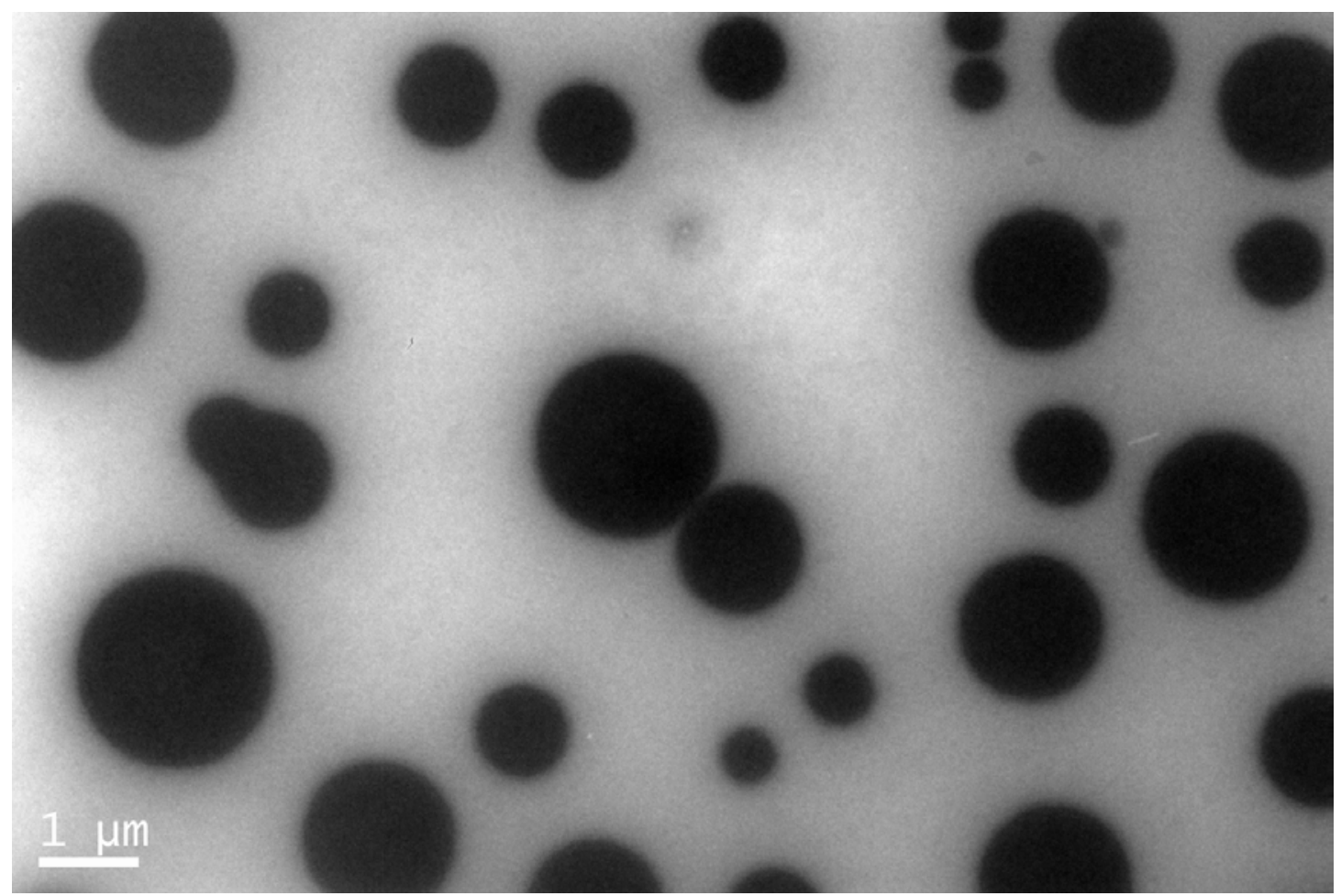

Figure 5-3: A TEM micrograph of germanium nanoparticles obtained from experiment 2 
As may be seen in Figure 5-3, spherical very large particles were observed in the TEM image. The particles were poly dispersed with size range of $500 \mathrm{~nm} \sim 2 \mu \mathrm{m}$. The EDS analysis showed around 4000 counts from germanium $\mathrm{L}_{\alpha}$ ray and also 1700 counts from germanium $\mathrm{K}_{\alpha}$ ray. It indicates that the nanoparticles were composed mainly of germanium. 2200 counts of chlorine $\mathrm{K}_{\alpha}$ ray were also detected which is possibly from the surface of the nanoparticles or remaining lithium chloride salt. The results from experiment 1 and 2, indicate that most of the germanium nanoparticles formed by the reaction mainly stayed in the solution.

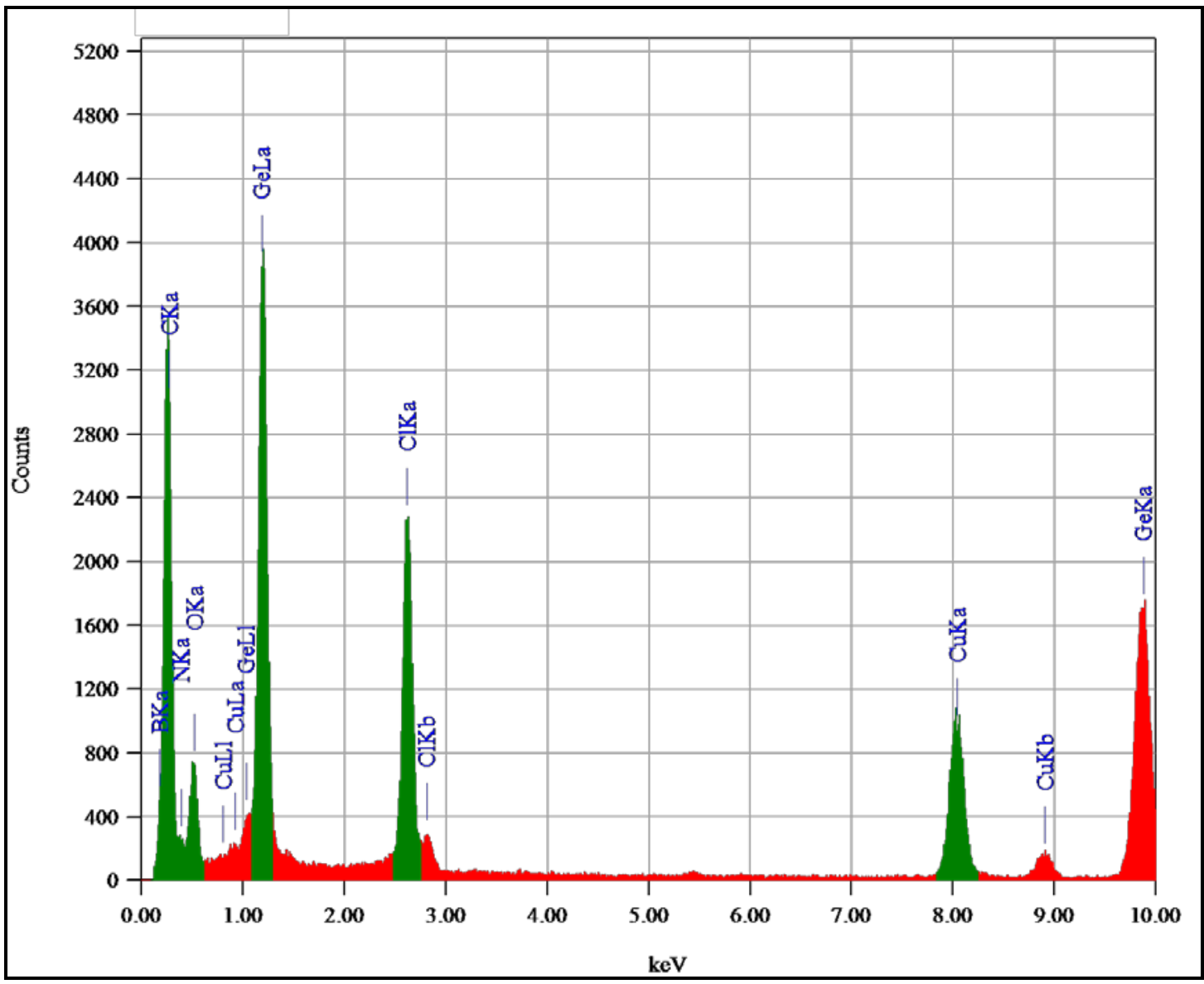

Figure 5-4: EDS spectrum of germanium nanoparticles obtained from experiment 2 


\section{5-1-2. Syntheses of germanium quantum dots capped with 1,5-hexadiene}

\section{Experiment 3}

To form hydrophobic surface, 1,5-hexadiene was chosen as a capping agent. Hydrophobic surface enables liquid-phase separation in which polar impurities such as lithium chloride can be removed from the quantum dots. As silicon quantum dots reported in chapter 3 , one of the terminal double bond of the 1,5-hexadiene is expected to attach to the surface of the quantum dot and the other one remaining at the terminal end. The remaining double bond can be converted to hydroxyl group or other functional group for the purpose of biological applications.

\section{Experimental}

This experiment was carried out as the same procedure of experiment 2. $0.59 \mathrm{~g}$ of pentaethylene glycol monododecyl ether $\left(\mathrm{C}_{12} \mathrm{E}_{5}\right), 0.2 \mathrm{~mL}(0.002 \mathrm{~mol})$ of germanium tetrachloride $\left(\mathrm{GeCl}_{4}\right)$, and $4.0 \mathrm{~mL}$ of $1.0 \mathrm{M}$ lithium aluminiumhydride were used in this experiment. Surface passivation was conducted by UV-irradiation method with $4.0 \mathrm{~mL}$ of 1,5-hexadiene for 4 hours. After the reaction the solution was concentrated and purified by size exclusion column chromatography (LH20). Fluorescent fractions were collected and concentrated. 


\section{Results}

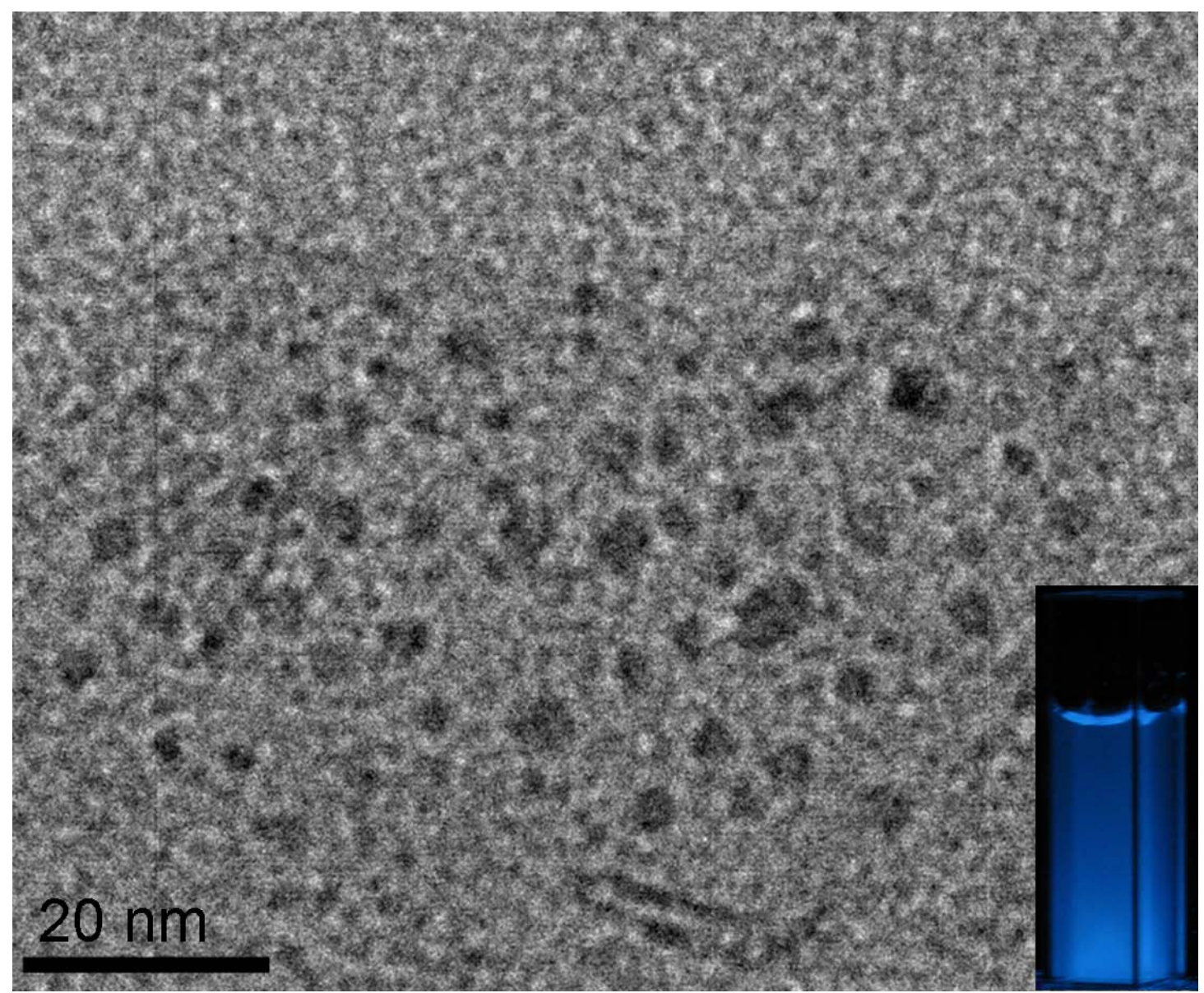

Figure 5-5: A TEM micrograph of germanium nanoparticles obtained from experiment 3

As seen in Figure 5-5, aggregates were observed from TEM micrograph. The shapes were almost spherical with an average size of $3.1 \pm 1.0 \mathrm{~nm}$. EDS analysis showed the existence of germanium. This indicates that the nanoparticles were possibly composed of germanium. This sample gives blue luminescence as seen in the inset of the Figure 5-5.

\section{Discussion}

The results from experiment 2 and 3 of the microemulsion syntheses showed the evidence of germanium nanoparticles in TEM micrograph and EDS analysis. There was a large difference in the average particle size between these two experiments. 
There some possible reasons can be listed. One of them is the purification methods. Experiment 2 was purified by liquid-phase separation which only dependent on surface polarity. On the other hand, the sample of experiment 3 was purified by size exclusion column chromatography. The majority of the particles observed from experiment 2 were much larger than the exciton Bohr radius of germanium, however these particles do not fluoresce. Therefore in the experiment 3, the large size nanoparticles were separated from the small size nanoparticles which give off fluorescence in the column. Another reason could be due to the reducing agents. Prabakar et. al. reported size controlled synthesis of germanium nanoparticles by using various reducing agents. ${ }^{15}$ As reducing agent, lithium aluminiumhydride is stronger than lithium borohydride therefore the reaction using lithium aluminiumhydride as a reducing agent proceeds vigorously and quickly due to the strong reducing capability of lithium aluminiumhydride. As a result of this rapid reduction there is a quick depletion of monomers with few monomers remaining for further growth of the nanoparticles. On the other hand, lithium borohydride has lower reactivity compared to lithium aluminiumhydride therefore, it produced larger nanoparticle sizes.

\section{5-2. High temperature synthesis of germanium quantum dot}

Since the microemulsion syntheses did not produce reasonable germanium quantum dots, high temperature syntheses of germanium quantum dots were investigated. High temperature synthesis of germanium quantum dots has been previously introduced by Dr. Jamie Warner using organogermane. ${ }^{20}$ The advantage of this synthesis is that this reaction allows absence of surfactant which facilitates sample purification. In addition, since strong hydride reducing agents were used in microemulsion system, there is a possibility of producing highly reactive gas, germane gas $\left(\mathrm{GeH}_{4}\right)$, which spontaneously combusts when in contact with the oxygen. ${ }^{21}$ In this section, the effect of different solvents and capping molecules at different temperature was investigated. All the capping molecules were chosen on the aim of biological applications. All the reactions were conducted under nitrogen atmosphere. Triphenyl chlorogermane was used as a precursor and sulfur was used to dephenylation. The reaction mechanism 
was incorporated by the publication from Schumann et.al. ${ }^{22}$ The possible reaction mechanism is shown in scheme $5-2-1$.

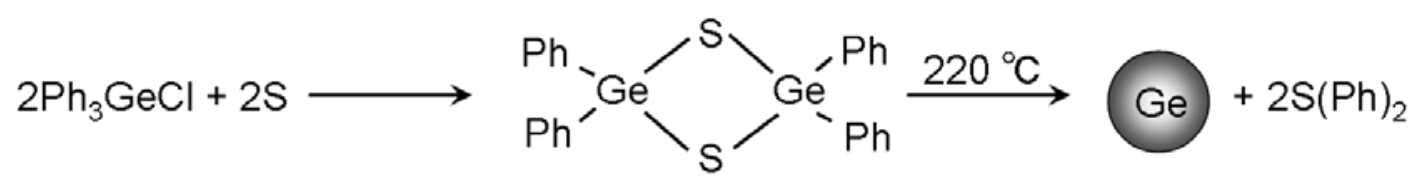

Scheme 5-2-1: Reaction scheme of the high temperature synthesis of the germanium quantum dot

\section{5-2-1. Reaction of triphenylgermanium chloride and sulfur in hexadecylamine (HDA ) with oleic acid capping at $260{ }^{\circ} \mathrm{C}$}

\section{Experiment 4}

In this experiment the reaction of triphenylgermanium chloride and sulfur in hexadecylamine (HDA) was conducted at $260{ }^{\circ} \mathrm{C}$. Oleic acid was chosen as a capping molecule. Oleic acid is mono-unsaturated acid and is found in various animal or vegetable sources. It is widely used in cosmetic items therefore it can be expected to be biocompatible. ${ }^{23}$ The carboxylate anions are expected to attach to the surface of the germanium quantum dots and produce hydrophobic germanium quantum dots. It was expected that sulfur could help the dephenylation of the precursor and form an intermediate trimer with germanium. This intermediate compound decompose above $220{ }^{\circ} \mathrm{C}$ to form elemental germanium with diphenylsulfur as a side product. ${ }^{22}$

\section{Experimental}

$4.5 \mathrm{~g}$ of hexadecylamine (HDA) and $0.05 \mathrm{~g}$ of triphenylgerminum chloride were weighed into a three neck flask and heated up until melting. Impurities came out as a white fume after the temperature had reached $200{ }^{\circ} \mathrm{C}$. After removal of the fumes, the solution of sulfur in hexadecylamine (HDA) was injected into the three neck flask. The sulfur solution was prepared by weighing $0.005 \mathrm{~g}$ of sulfur and $1.5 \mathrm{~g}$ of HDA heated up until melting. The colour of the solution started to change dramatically into dark brown after a few minutes of sulfur injection. After the colour change was observed, $2 \mathrm{~mL}$ of oleic acid was injected into the solution. A few seconds after the 
oleic acid injection, the solution cleared and after being left for about 40 minutes the solution was slowly cooled down to room temperature. Purification was conducted by washing with ethanol and centrifugation. A dark brown cloudy solution was obtained.

\section{Results}

From the TEM analysis no nanoparticles of spherical shape were observed. The EDS analysis showed the aggregates contained germanium and sulfur. No SAED was obtained, therefore it was confirmed that the aggregates were not crystalline germanium. This might be due to the temperature being not high enough to produce crystalline germanium. The sulfur injection temperature of the solution might have decreased.

\section{5-2-2. Reaction of triphenylgermanium chloride and sulfur in Oleylamine with mercaptoundecanoic acid capping at $290{ }^{\circ} \mathrm{C}$}

\section{Experiment 5}

Mercaptoundecanoic acid has a thiol group and a carboxyl group at each terminal. Both functional groups have the potential to attach to the surface of the quantum dots through sulfur or oxygen atoms and leave the other functional group at the other terminal end. Either functional group left on the surface could be used to attach biological molecules on to it.

\section{$\mathrm{Ge}-\mathrm{S}-\mathrm{CH}_{2}-\left(\mathrm{CH}_{2}\right)_{6}-\mathrm{CH}_{2}-\mathrm{COOH}$}

\section{$\mathrm{Ge}-\mathrm{OOC}-\mathrm{CH}_{2}-\left(\mathrm{CH}_{2}\right)_{6}-\mathrm{CH}_{2}-\mathrm{SH}$}

Figure 5-2-2: Possible structures of the germanium qunautm dots capped with mercaptoundecanoic acid 


\section{Experimental}

The experiment was carried out in the same procedure as experiment 4. In this experiment $7.5 \mathrm{~mL}$ of oleylamine was used as a solvent and $2.0 \mathrm{~mL}$ was used to dissolve sulfur. Also $0.1 \mathrm{~g}$ of mercaptoundecanoic acid was used as a capping agent. The reaction temperature was $290{ }^{\circ} \mathrm{C}$.

\section{Results}

The sample was characterised by TEM and surface molecules were analysed by FTIR.

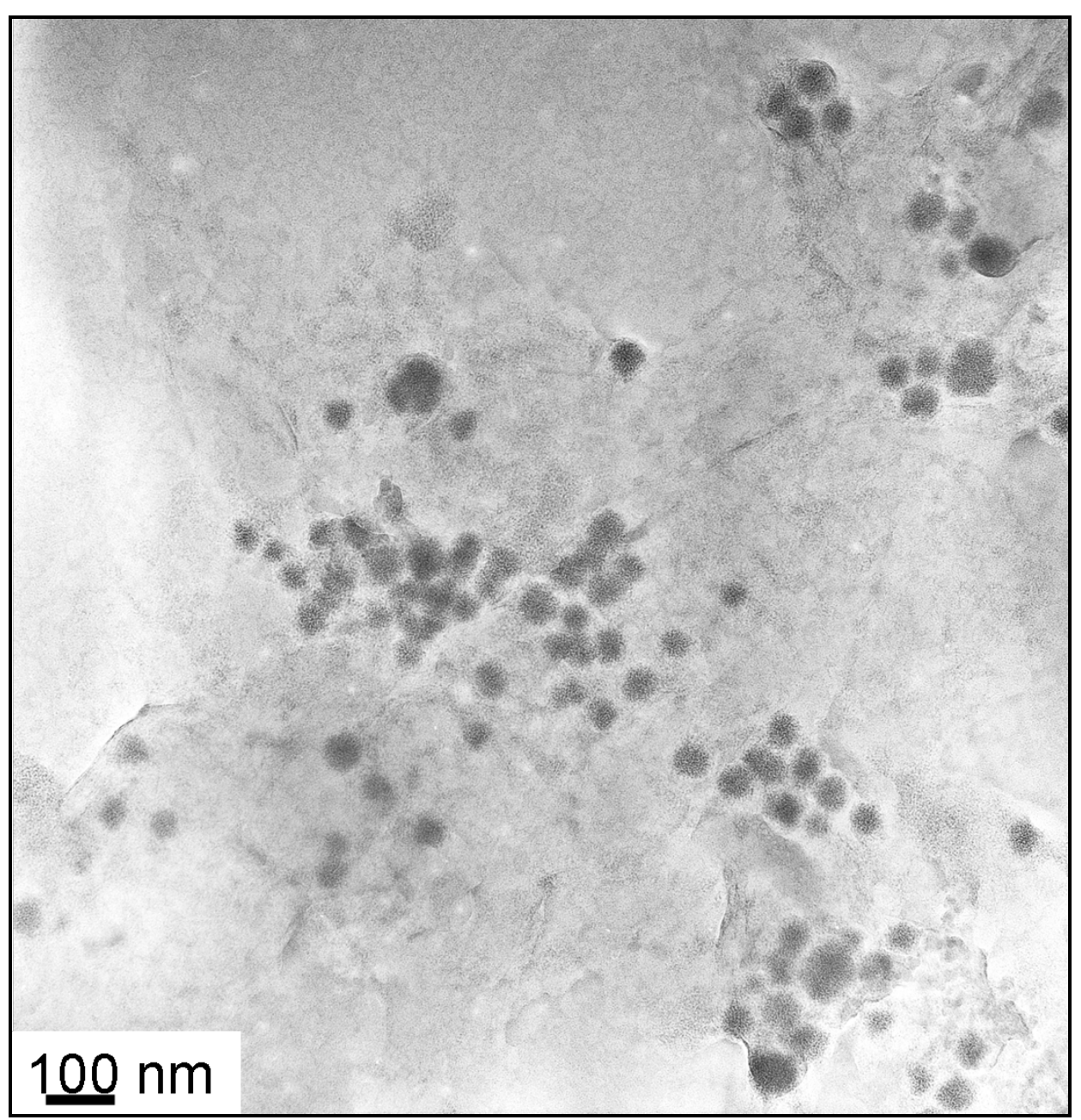

Figure 5-6: A low resolution TEM micrograph of the sample obtained from experiment 5

As seen in Figure 5-6, some aggregates were observed. The shapes of the aggregates were almost spherical within average size of $40.5 \pm 5.7 \mathrm{~nm}$. Probably due to impurities from sulfer, the nanoparticles were vaguely outlined. Lattice fringes could 
not be observed either, however the EDS analysis confirmed the presence of germanium. The result indicates that the aggregates were composed of mainly germanium.

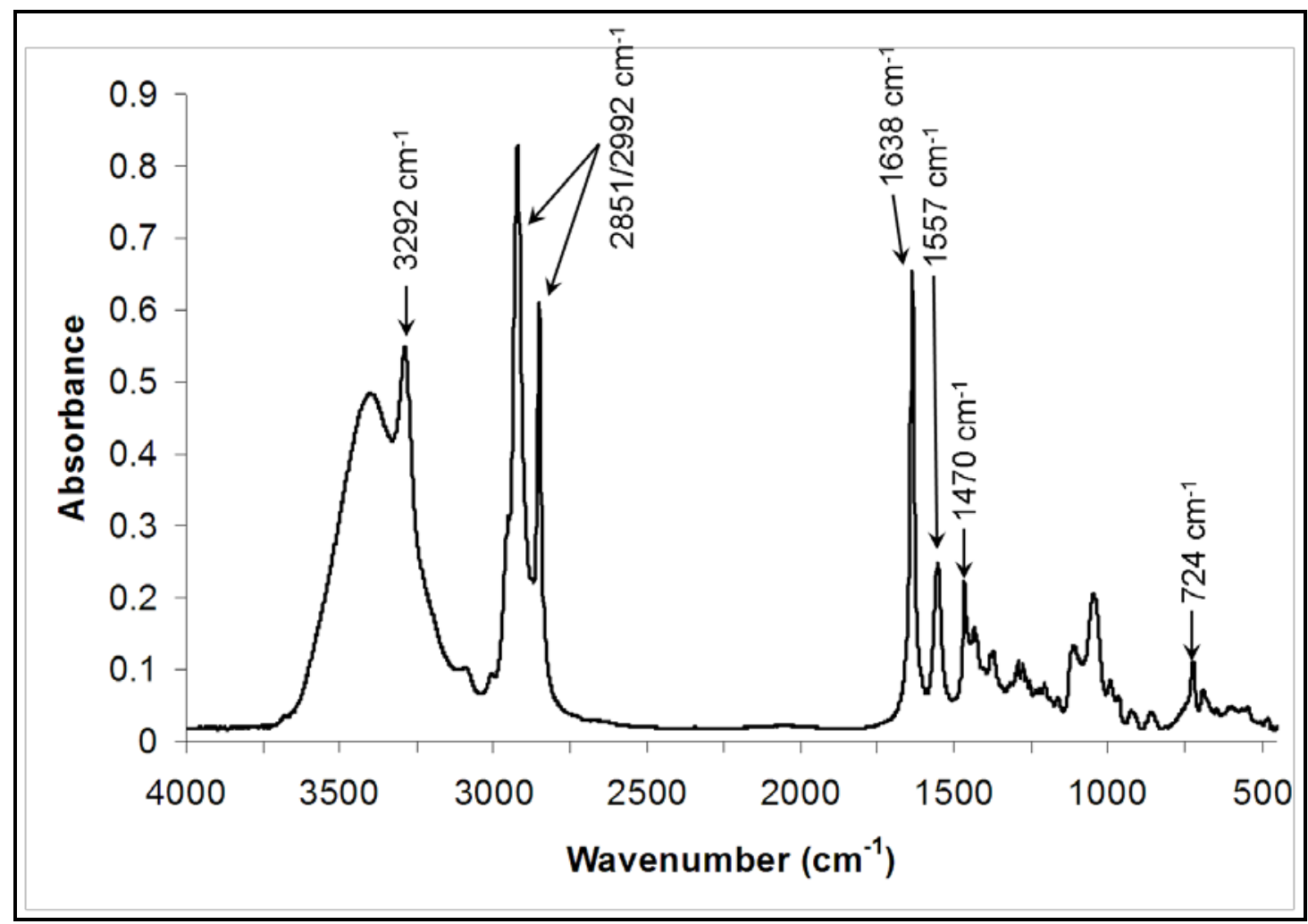

Figure 5-7: FTIR spectrum of germanium quantum dots capped with mercaptoundecanoic acid

As may be seen in Figure 5-7, a strong $\mathrm{C}=\mathrm{O}$ stretching from the carboxyl group was observed at $1638 \mathrm{~cm}^{-1}$. Also $\mathrm{C}-\mathrm{O}(\mathrm{H})$ stretching from the carboxyl group can be observed at $1557 \mathrm{~cm}^{-1}$. The peaks at $724 \mathrm{~cm}^{-1}$, and $2851 \mathrm{~cm}^{-1} / 2992 \mathrm{~cm}^{-1}$ corresponded to $\mathrm{CH}_{2}$ in plane bending and $\mathrm{CH}_{2}$ symmetrical, asymmetrical stretching respectively. $\mathrm{CH}_{2}$ plane scissoring was seen at $1470 \mathrm{~cm}^{-1}$ and $\mathrm{O}-\mathrm{H}$ stretching from the carboxyl group can be seen at $3292 \mathrm{~cm}^{-1}$. However the peak corresponding to $\mathrm{S}-\mathrm{H}$ from the mercapto group around $2500 \mathrm{~cm}^{-1}$ to $2600 \mathrm{~cm}^{-1}$ could not be observed. From the result it seemed that this capping agent was attached to germanium clusters through sulphur atoms from the mercapto group. 


\section{5-2-3. Reaction of triphenylgermanium chloride and sulfur in Oleylamine with octadecene capping at $300{ }^{\circ} \mathrm{C}$}

\section{Experiment 6}

Octadecene has a boiling point of $315{ }^{\circ} \mathrm{C}$ and has the terminal carbon double bond which has the potential to form a covalent bond on the germanium surface. Passivation of germanium nanoparticles with Ge-C covalent bond gives very stable surface state to the nanoparticles which is required for biological applications. Since octadecene has simple molecular structure, it was suitable for the surface characterisation to find out the details of the surface molecules. The reaction was conducted at $300{ }^{\circ} \mathrm{C}$.

\section{$\mathrm{Ge}-\mathrm{CH}_{2}-\left(\mathrm{CH}_{2}\right)_{16}-\mathrm{CH}_{3}$}

Figure 5-2-2: Structure of the germanium quantum dot capped with octadecene

\section{Experimental}

The experiment was carried out in the same procedure as experiment 4. In this experiment $7.5 \mathrm{~mL}$ of oleylamine was used as a solvent and $2.0 \mathrm{~mL}$ was used to dissolve sulfur. Also $2 \mathrm{~mL}$ of octadecene was used as a capping agent. The reaction temperature was $300{ }^{\circ} \mathrm{C}$.

\section{Results}

The sample was characterised by TEM and surface molecules were analysed by FTIR. As may be seen in Figure 5-8, spherical aggregates were observed. Majority of the nanoparticles have the average size of $90.6 \pm 15.7 \mathrm{~nm}$. EDS analysis showed the nanoparticles were mainly composed of germanium, yet a very small sulfur peak was observed. 


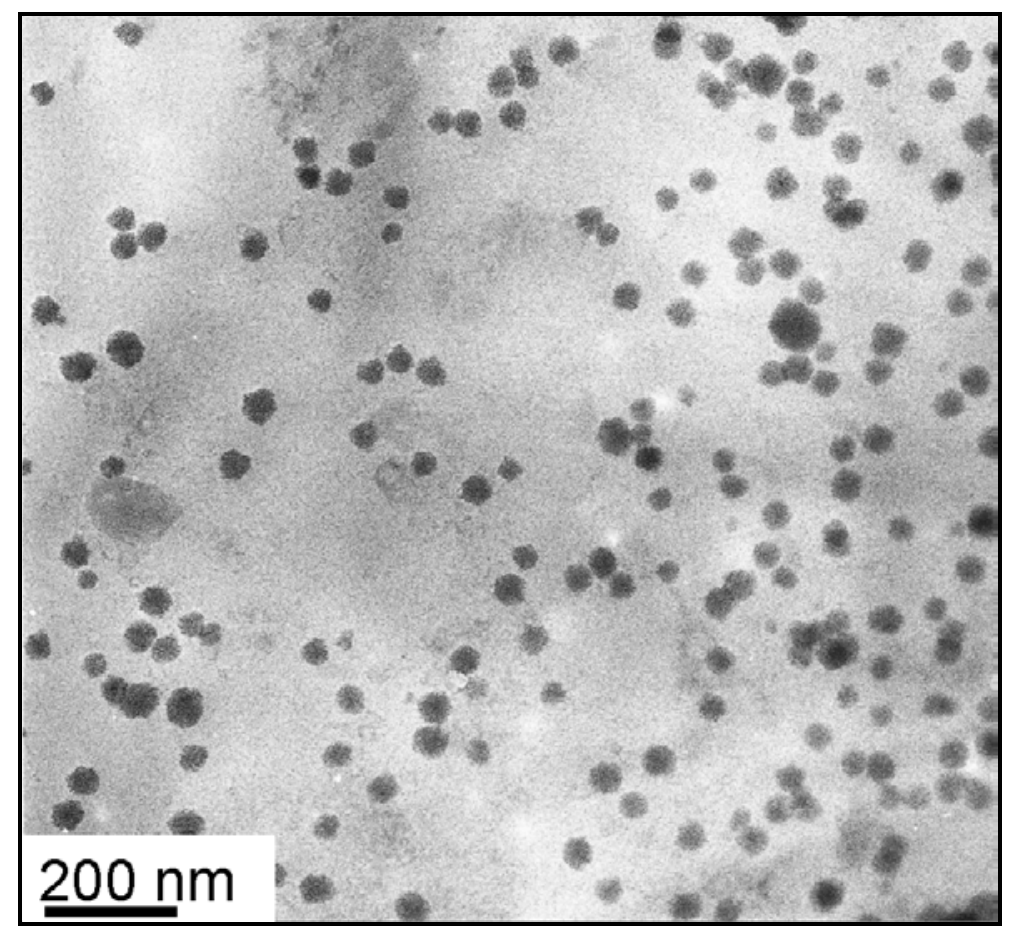

Figure 5-8: A TEM micrograph of the sample obtained from experiment 6

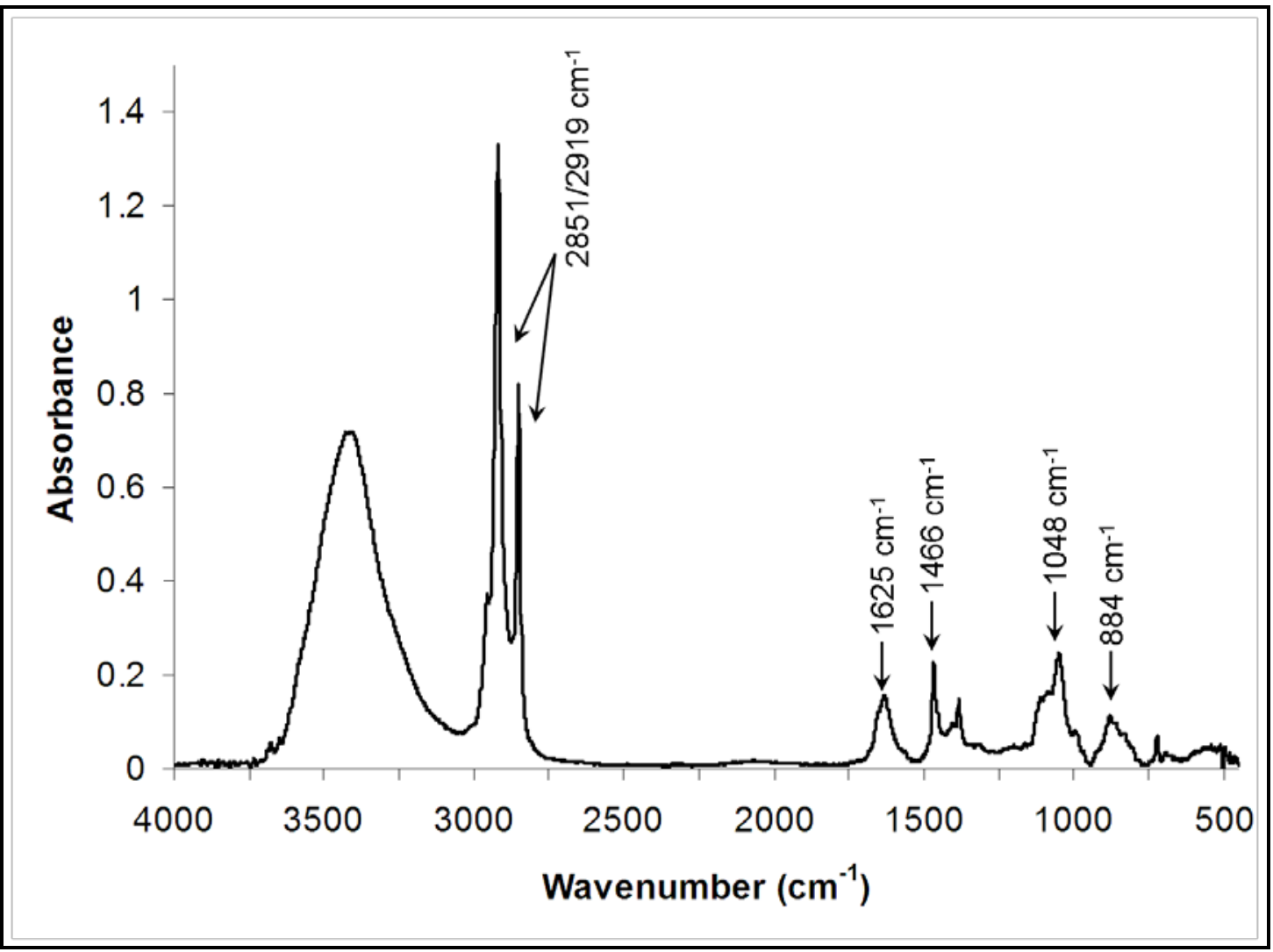

Figure 5-9: FTIR spectrum of the sample obtained from experiment 6 
As may be seen in Figure 5-9, $\mathrm{CH}_{2}$ plane scissoring from octadecene was seen at 1466 $\mathrm{cm}^{-1}$ and $\mathrm{CH}_{2}$ symmetrical and asymmetrical stretching can be seen at the peaks of $2851 \mathrm{~cm}^{-1} / 2919 \mathrm{~cm}^{-1}$. The small peak at $884 \mathrm{~cm}^{-1}$ possibly corresponds to Ge-C stretching. ${ }^{8}$ Usually Ge-C stretching can be seen at $830 \mathrm{~cm}^{-1}$, although the Ge-C stretch for the surface of the germanium nanoparticles can be shifted to higher frequency. ${ }^{8}$ It shows the attachment of the capping molecule to the germanium nanoparticles. The peaks at $1625 \mathrm{~cm}^{-1}$ and $3400 \mathrm{~cm}^{-1}$ can be assigned to the vibration of water molecules. ${ }^{24}$ From this result the attachment of octadecene to the surface of germanium quantum dots can be seen.

\section{5-2-4. Reaction of triphenylgermanium chloride and sulfur in Oleylamine with folic acid capping at $290{ }^{\circ} \mathrm{C}$}

\section{Experiment 7}

Folic acid, also known as vitamin $\mathrm{B}_{9}$ and is one of the water-soluble vitamins. Folic acid has a structure binding glutamic acid and p-aminobenzoic acid onto pritedine. It itself is not biologically active however after conversion to tetrahydrofolate through dihydrofolic acid in liver it works as coenzyme. ${ }^{25,26}$ The structures of folic acid and tetrahydrofolate are presented in Figure 5-10. 


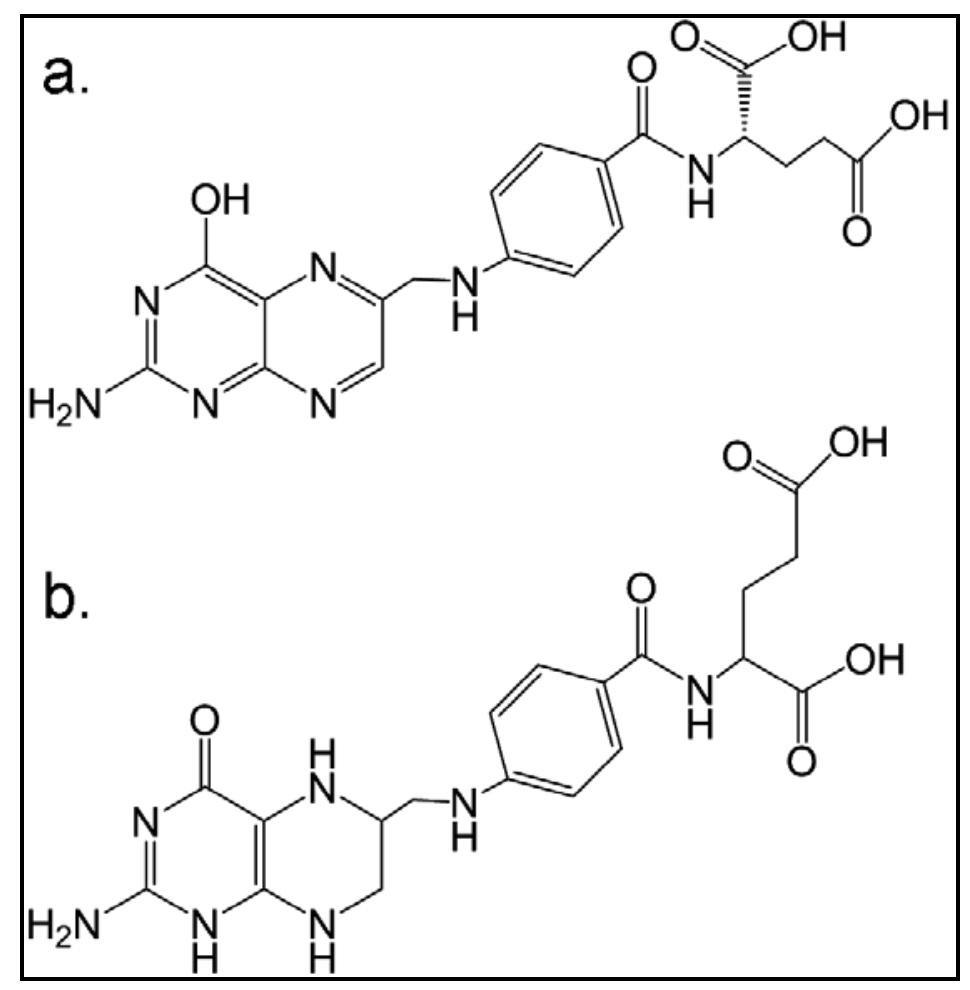

Figure 5-10: Structures of folic acid (a) and tetrahydrofolate (b)

Folic acid and folate inclusive are essential to numerous bodily functions ranging from nucleotide biosynthesis to the remethylation of homocysteine. It is especially important during periods of rapid cell division and growth. Folic acid is necessary to produce healthy red blood cells and to prevent anaemia.

Folic acid has melting point of $250{ }^{\circ} \mathrm{C}$ and is soluble in oleylamine. To avoid decomposition folic acid was injected into the reaction mixture after the temperature dropped down to $240{ }^{\circ} \mathrm{C}$ from the highest point, $290{ }^{\circ} \mathrm{C}$. Attaching folic acid to germanium quantum dots makes them water soluble and safe in organs.<smiles>COC(=O)[C@H](CCC(=O)Oc1ccccc1)NC(=O)c1ccc(NCc2cnc3nc(N)nc(O)c3n2)cc1</smiles>

Figure 5-2-3: Structure of the germanium qunautm dot capped with folic acid 


\section{Experimental}

The experiment was carried out in the same procedure as experiment 4. In this experiment $7.5 \mathrm{~mL}$ of oleylamine was used as a solvent and $2.0 \mathrm{~mL}$ was used to dissolve sulfur. Also $0.2 \mathrm{~g}$ of folic acid dissolved in $2 \mathrm{~mL}$ of oleylamine was used as a capping agent. The reaction temperature was $290{ }^{\circ} \mathrm{C}$.However since folic acid decomposes at $250{ }^{\circ} \mathrm{C}$ the solution was cooled down to $240{ }^{\circ} \mathrm{C}$ before injecting folic acid.

\section{Results}

The sample was characterized by TEM and EDS. Figure 5-11 shows a TEM micrograph of the germanium nanoparticles capped with folic acid.

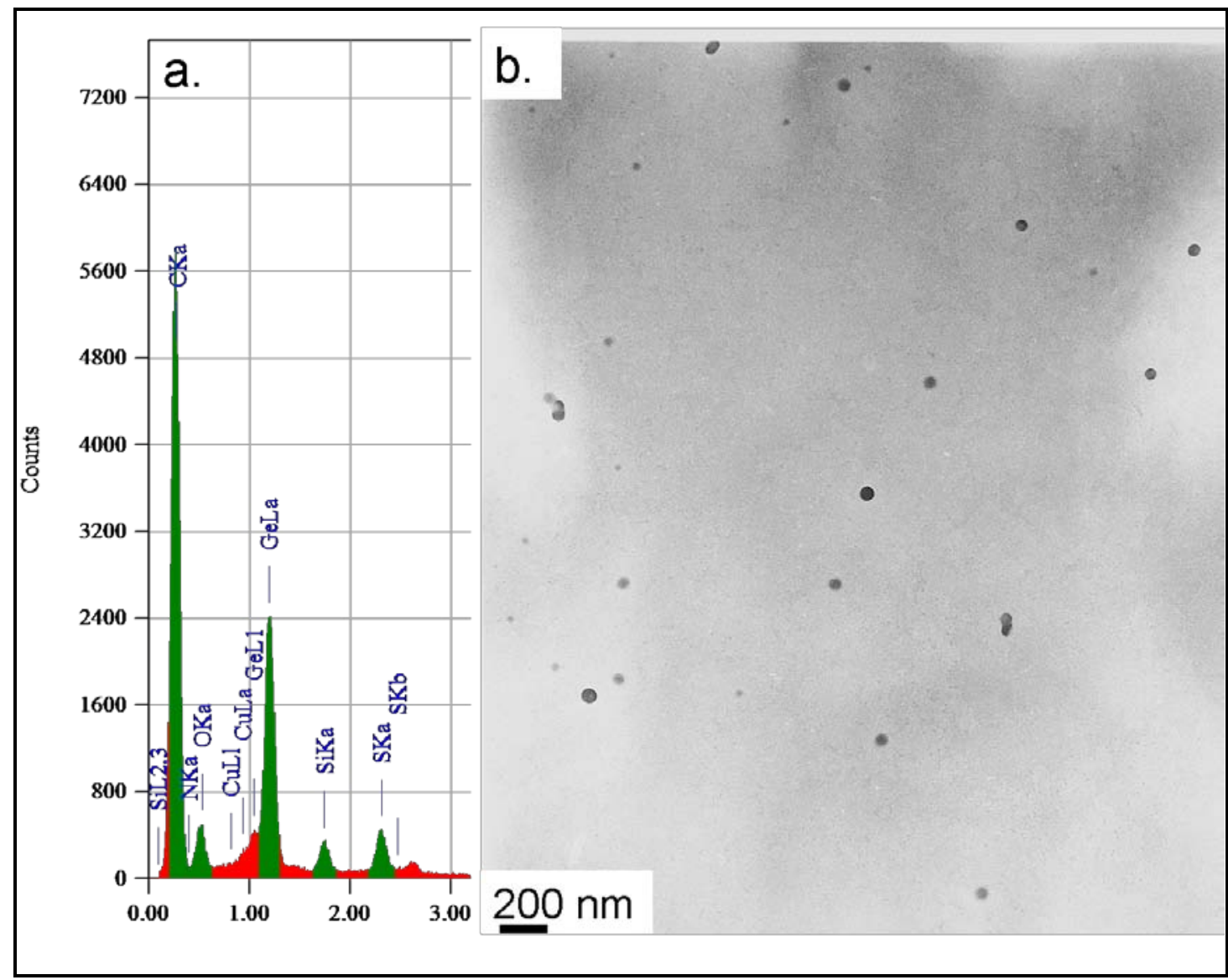

Figure 5-6: A EDS spectrum of the sample obtained from experiment 7 (a), and TEM micrograph of the same area (b)

As seen in Figure 5-11, spherical nanoparticles with sizes of $41.2 \pm 6.5 \mathrm{~nm}$ were observed. The EDS spectrum showed around 2400 counts of germanium $\mathrm{L}_{\alpha}$ ray. Since there hardly any sulfur peak was observed in the EDS spectrum the nanoparticles 
were composed only of germanium. The surface molecules were characterized by FTIR spectroscopy.

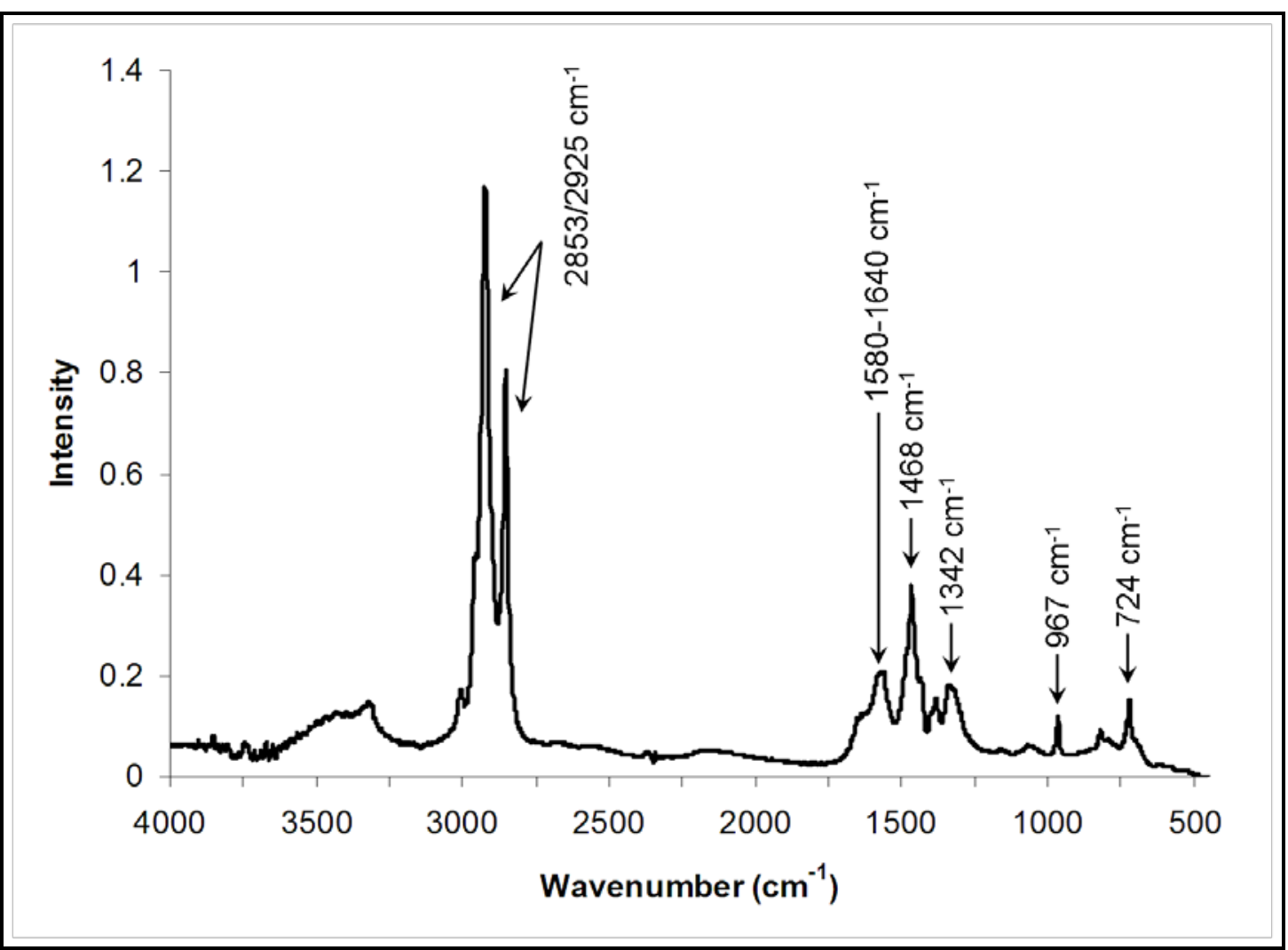

Figure 5-7: FTIR spectrum of germanium quantum dots capped with folic acid

In the FTIR spectrum, $\mathrm{CH}_{2}$ in plane bending and $\mathrm{CH}_{2}$ symmetrical, asymmetrical stretching can be seen at the peaks of $724 \mathrm{~cm}^{-1}, 2853 / 2925 \mathrm{~cm}^{-1}$ respectively. The peak corresponding to $\mathrm{C}=\mathrm{O}$ stretching from the carboxyl group can be seen at 1640 $\mathrm{cm}^{-1}$ in this spectrum which slightly shifted to lower frequency compared to free folic acid. It is possibly due to the attachment to the germanium nanoparticles. Meanwhile, the broad peak at $916 \mathrm{~cm}^{-1}$ which can be seen in FTIR spectrum of free folic acid cannot be seen in this spectrum. It corresponds to $\mathrm{O}-\mathrm{H}$ out of plane bending of inter molecular hydrogen bond between two carboxyl groups. This suggests that folic acid was attached to the surface of the germanium quantum dots through oxygen atoms from the $\mathrm{OH}$ group of the carboxyl group. $\mathrm{CH}_{2}$ plane scissoring was seen at $1468 \mathrm{~cm}^{-1}$. 


\section{5-2-5. Reaction of triphenylgermanium chloride and sulfur in Trioctylamine with 11-dodecen 1-ol capping at $300{ }^{\circ} \mathrm{C}$}

\section{Experiment 8}

Germanium quantum dots capped with 11-dodecen 1-ol were synthesized to produce hydroxyl group terminated germanium quantum dots. 11-dodecen 1-ol has its boiling point at $238{ }^{\circ} \mathrm{C}$ and possesses a terminal double bond and a terminal hydroxyl group. Trioctylamine was used as the solvent. The terminal hydroxyl group can be modified to attach biological molecules to the surface of the germanium quantum dots.

\section{$\mathrm{Ge}-\mathrm{CH}_{2}-\left(\mathrm{CH}_{2}\right)_{10}-\mathrm{CH}_{2}-\mathrm{OH}$}

Figure 5-2-4: Structure of the germanium quantum dot capped with 11-dodecen-1-ol

\section{Experimental}

The experiment was carried out in the same procedure as experiment 4. In this experiment $7.5 \mathrm{~mL}$ of trioctylamine (TOA) was used as a solvent and $2.0 \mathrm{~mL}$ was used to dissolve sulfur. Also $3 \mathrm{~mL}$ of 11-dodecen-1-ol was used as a capping agent. The reaction temperature was $300{ }^{\circ} \mathrm{C}$. In this experiment after injecting $2 \mathrm{~mL}$ of 11 dodecen-1-ol at $300{ }^{\circ} \mathrm{C}$ the solution was cooled down to $230{ }^{\circ} \mathrm{C}$ and another $1 \mathrm{~mL}$ of 11-dodecen-1-ol was injected.

\section{Results}

The sample was characterized by TEM and the surface molecules were analyzed by FTIR.

As seen in Figure 5-13, spherical nanoparticles were observed from the TEM micrograph with the average sizes of $27.1 \pm 5.9 \mathrm{~nm}$. EDS analysis showed the existence of germanium. 


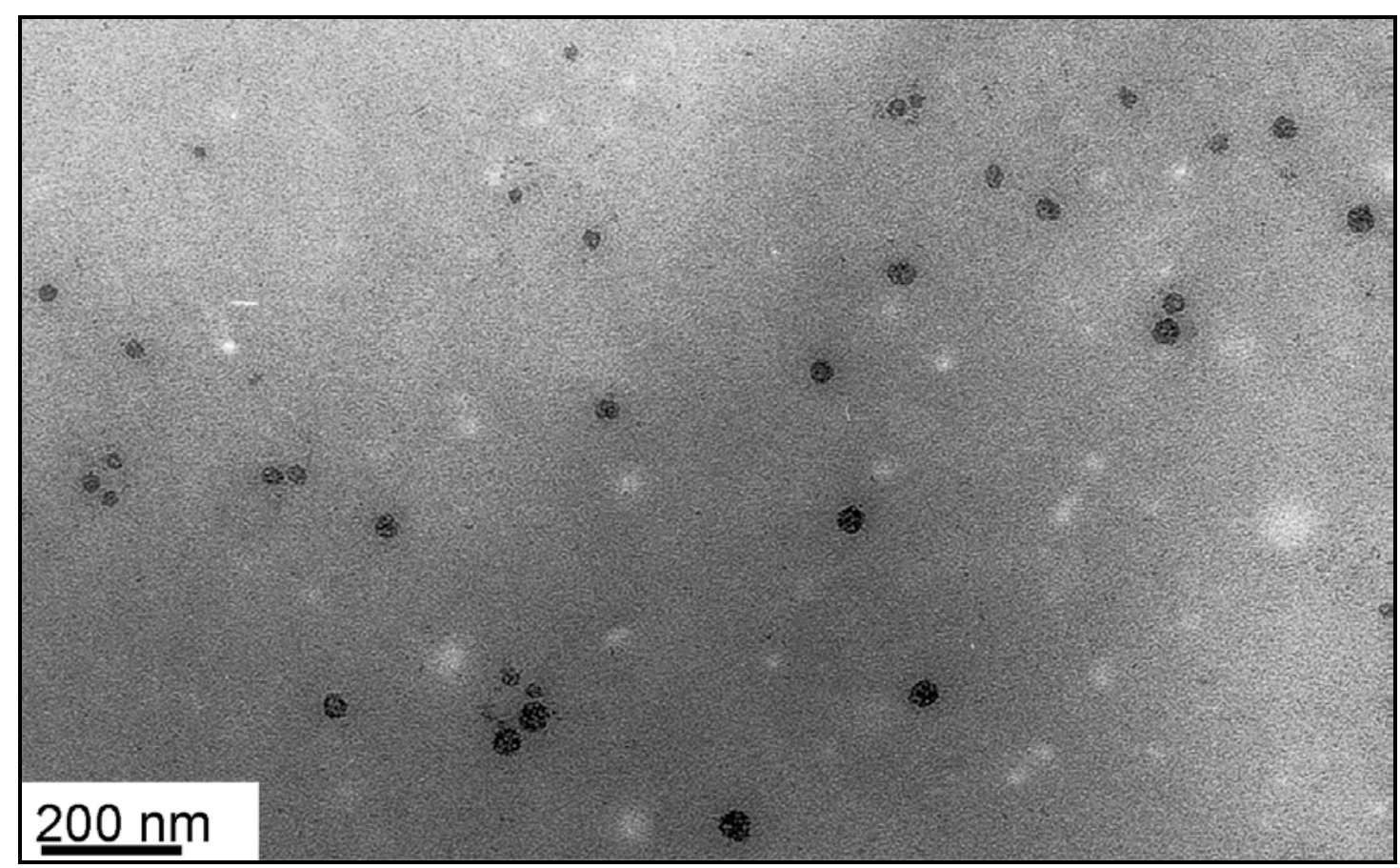

Figure 5-8: A low resolution TEM micrograph of germanium quantum dots capped with 11-dodecen-1-ol.

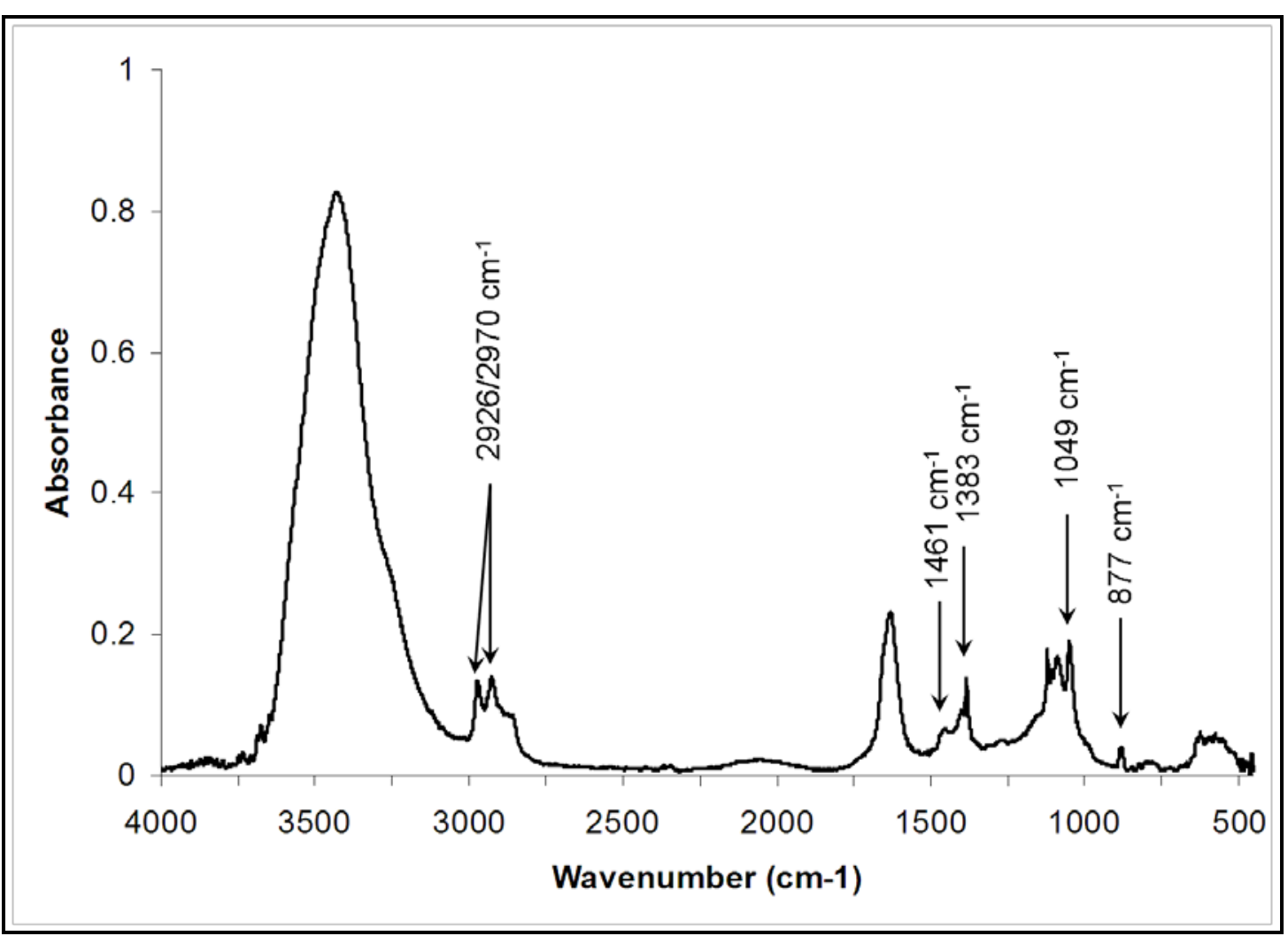

Figure 5-9: FTIR spectrum of the sample obtained form experiment 8 
In the FTIR spectrum, the peak corresponding to Ge-C bond can be seen at $877 \mathrm{~cm}^{-1}$. Also the peaks corresponding to $\mathrm{O}-\mathrm{H}$ in plane bending around $1378 \mathrm{~cm}^{-1}$ and 1461 $\mathrm{cm}^{-1}$ could be seen. The peak from C-O stretching can be seen at $1049 \mathrm{~cm}^{-1} \cdot \mathrm{CH}_{2}$ in plane bending and $\mathrm{CH}_{2}$ symmetrical, asymmetrical stretching from the capping agent were seen at $2926 \mathrm{~cm}^{-1}$ and $2970 \mathrm{~cm}^{-1}$. The broad peaks around $1600 \mathrm{~cm}^{-1}$ and 3400 $\mathrm{cm}^{-1}$ could be from the vibration of water molecules. ${ }^{24}$ Since clear peak of O-H bond could be seen in the spectrum, probably the terminal carbon double bond was attached to the germanium atom on the surface.

\section{5-2-6. Reaction of triphenylgermanium chloride and sulfur in Oleylamine with methyl 10-undecenoate capping at $290{ }^{\circ} \mathrm{C}$}

\section{Experiment 9}

Germanium quantum dots capped with methyl 10-undecenoate were synthesized to produce germanium quantum dots with ester groups on the surface. The surface ester groups can therefore later on be converted to carboxyl groups by adding a base to functionalise the quantum dots. Also it can react with the amine group to form an amide bond which will open up possibilities to attach bio-friendly molecules. Methyl 10-undecenoate has a boiling point of $245{ }^{\circ} \mathrm{C}$ which suits the high temperature synthesis.

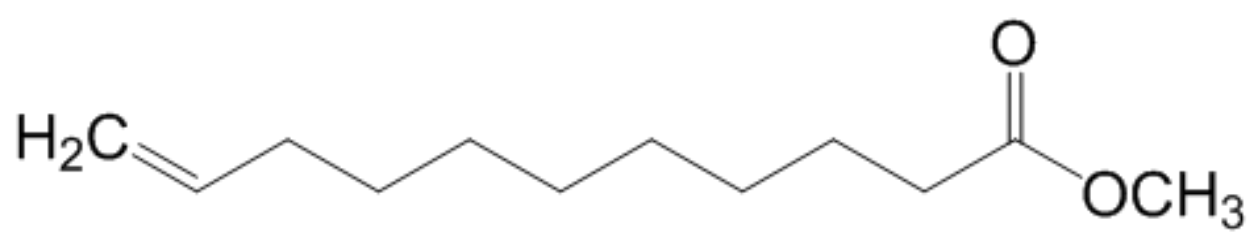

\section{$\mathrm{Ge}-\mathrm{CH}_{2}-\left(\mathrm{CH}_{2}\right)_{9}-\mathrm{COOCH}_{3}$}

Figure 5-10: Structure of free methyl 10-undecenoate and the germanium quanutm dot capped twith 10-undecenoate

\section{Experimental}


The experiment was carried out in the same procedure as experiment 4. In this experiment $7.5 \mathrm{~mL}$ of trioctylamine (TOA) was used as a solvent and $2.0 \mathrm{~mL}$ was used to dissolve sulfur. Also $2 \mathrm{~mL}$ of methyl 10-undecenoate was used as a capping agent. The reaction temperature was $290{ }^{\circ} \mathrm{C}$. Since methyl 10 -undecenoate has its boiling point at $245^{\circ} \mathrm{C}$ the solution was cooled down to $240^{\circ} \mathrm{C}$ before injecting methyl 10-undecenoate.

\section{Results}

The sample was characterized by TEM, EDS and FTIR.

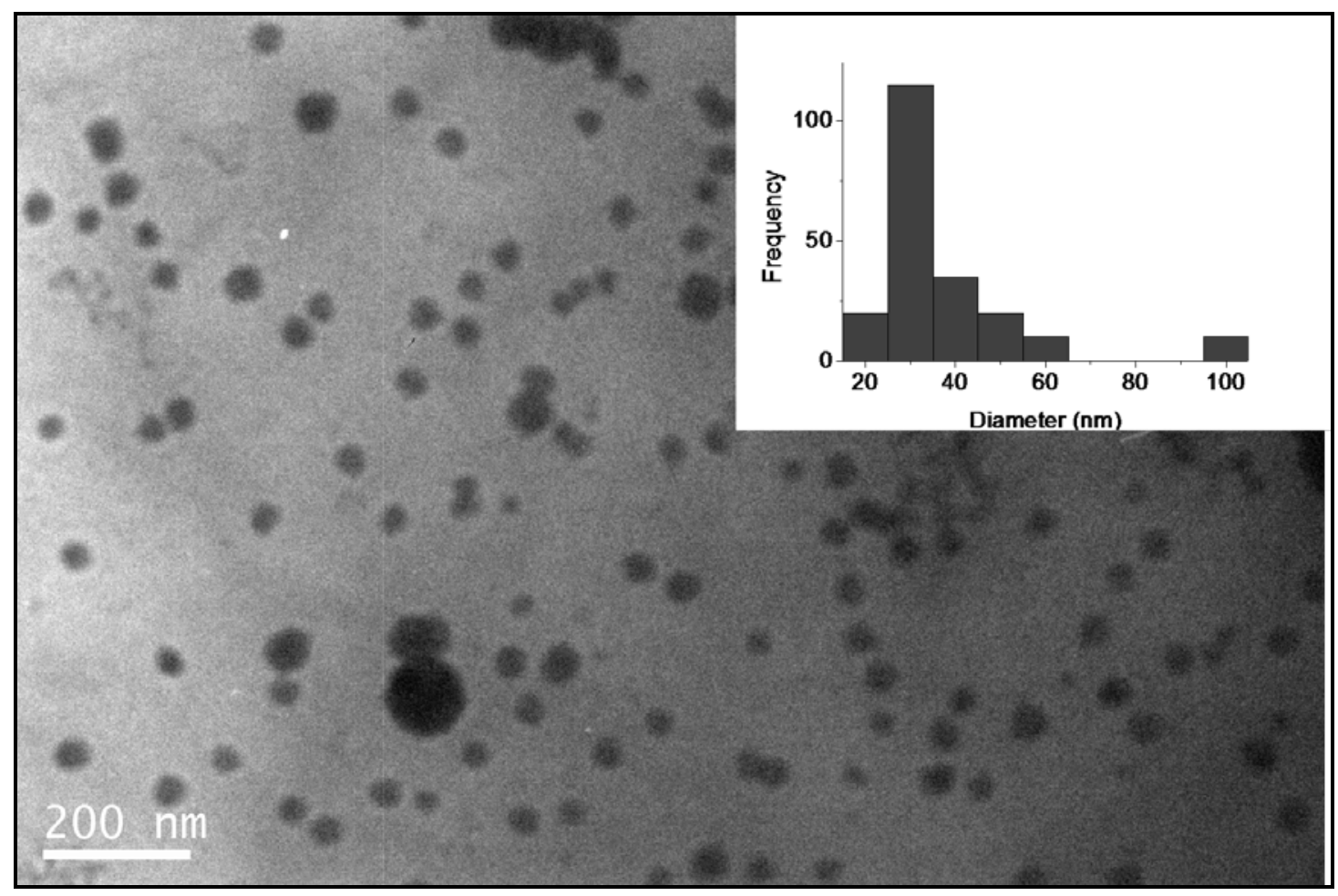

Figure 5-11: A TEM micrograph of germanium nanoparticles capped with methyl 10-undecenoate

A low resolution TEM micrograph is shown in Figure 5-16. Spherical nanoparticles were observed with average size of $42.8 \pm 7.5 \mathrm{~nm}$. The nanoparticles were relatively mono dispersed. EDS analysis showed that those nanoparticles were mainly composed of germanium. 


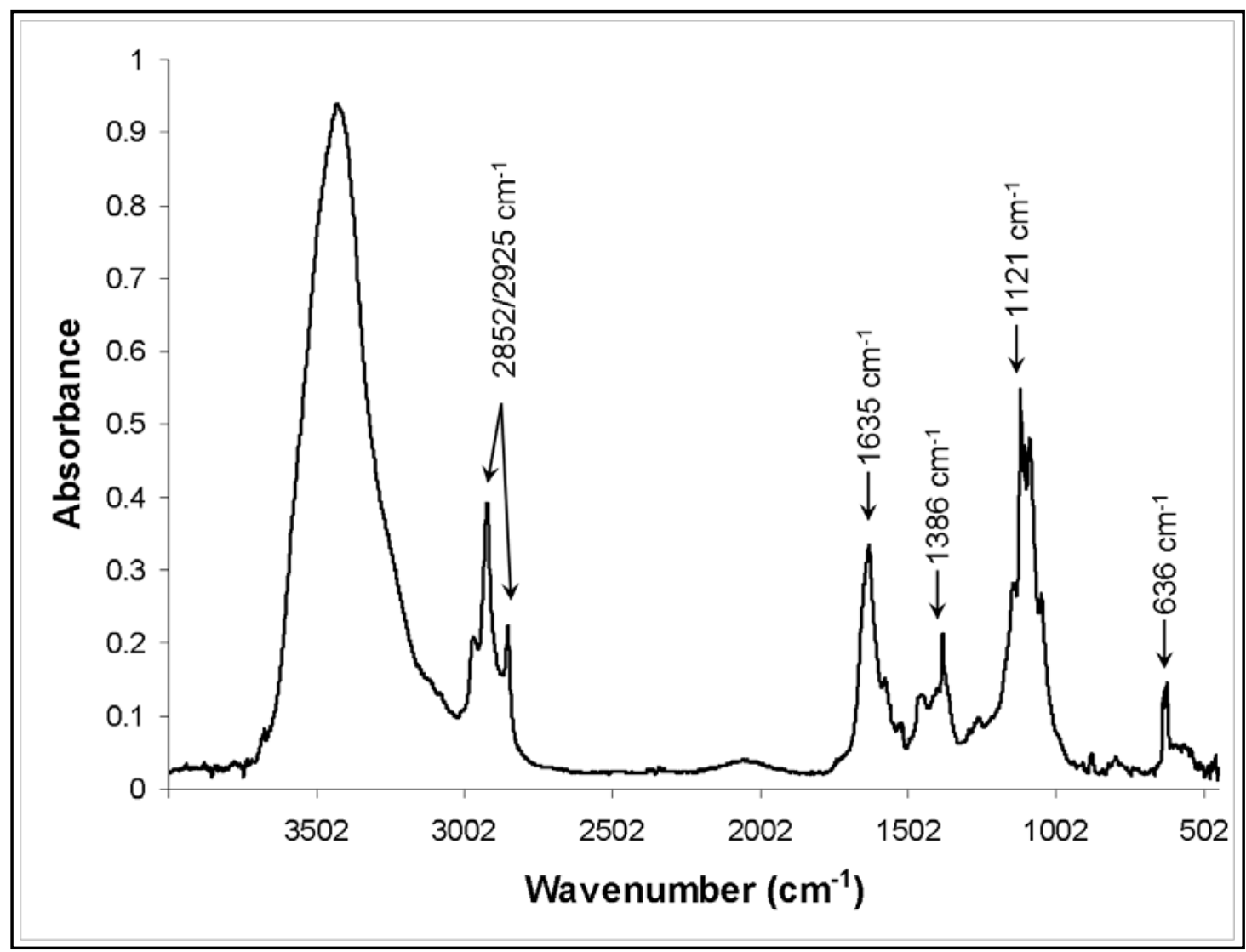

Figure 5-12: FTIR spectrum of germanium quantum dots capped with methyl 10undecenoate

From the FTIR spectrum, $\mathrm{CH}_{2}$ in plane bending and $\mathrm{CH}_{2}$ symmetrical, asymmetrical stretching from the capping agent were seen at $636 \mathrm{~cm}^{-1}, 2852 \mathrm{~cm}^{-1}$ and $2925 \mathrm{~cm}^{-1}$. Also $\mathrm{C}=\mathrm{O}$ stretching from the ester part was observed at $1636 \mathrm{~cm}^{-1}$. C-O stretching from the ester part can be seen at $1121 \mathrm{~cm}^{-1}$ as a strong peak. On the other hand the peak that corresponds to the terminal $\mathrm{C}=\mathrm{C}$ double bond cannot be seen. The result indicates the possibility of the terminal double bond of methyl 10-undecenoate being attached to the surface of the germanium quantum dots. 


\section{5-2-7. Reaction of triphenylgermanium chloride and sulfur in TOA (trioctyl amine) with oleic acid capping at $300{ }^{\circ} \mathrm{C}$}

\section{Experiment 10}

This reaction was conducted at $300{ }^{\circ} \mathrm{C}$ to make sure the sulfur and germanium intermediate decomposed. TOA which has higher purity compared to HDA was chosen as a solvent.

\section{$\mathrm{Ge}-\mathrm{OOC}-\mathrm{CH}_{2}-\left(\mathrm{CH}_{2}\right)_{7}-\mathrm{CH}=\mathrm{CH}-\left(\mathrm{CH}_{2}\right)_{7}-\mathrm{CH}_{3}$}

Figure 5-18: Structure of the germanium quantum dot capped with oleic acid

\section{Experimental}

The experiment was carried out in the same procedure as experiment 4. In this experiment $7.5 \mathrm{~mL}$ of trioctylamine (TOA) was used as a solvent and $2.0 \mathrm{~mL}$ was used to dissolve sulfur. Also $2 \mathrm{~mL}$ of oleic acid was used as a capping agent. The reaction temperature was $300{ }^{\circ} \mathrm{C}$.

\section{Results}

The sample was characterized by TEM, EDS and FTIR. Figure 5-19 shows TEM micrographs of the sample. 


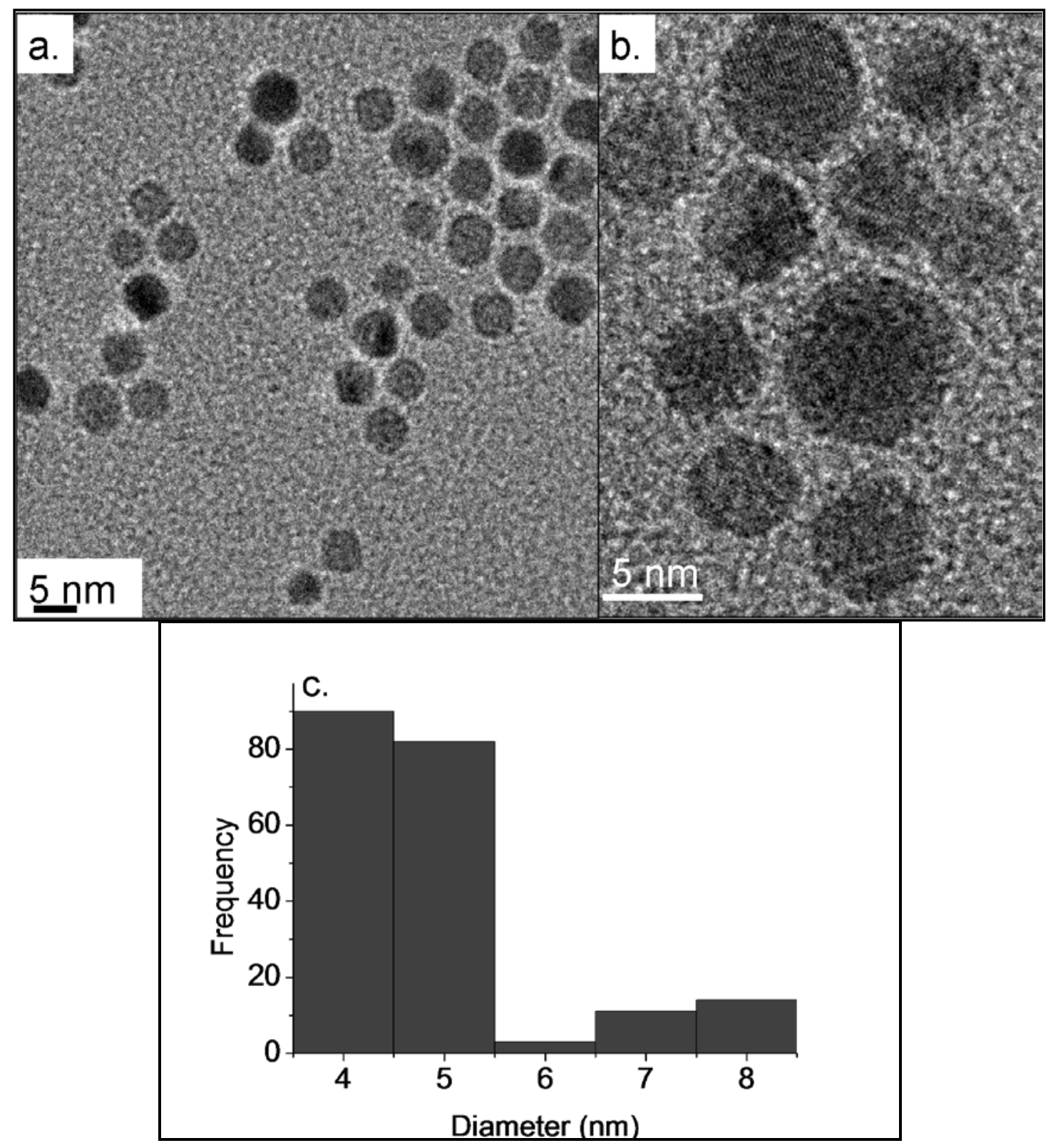

Figure 5-13: (a): a low resolution TEM micrograph of the nanoparticles obtained from experiment 10 (b): a high resolution TEM micrograph of the same sample (c): size distribution graph

TEM analysis showed relatively mono dispersed spherical nanoparticles in size of 5.6 $\pm 1.3 \mathrm{~nm}$. The nanoparticles were confirmed as crystalline nanoparticles since lattice fringes could be seen. From EDS analysis, the large germanium peak was observed with the $\mathrm{L}_{\alpha}$ count of 6100 . There also another peak of germanium $\mathrm{K}_{\alpha}$ ray with the counts of 2600 therefore it was confirmed that those nanoparticles were composed mainly of germanium. No significant amount of oxygen was detected therefore it was believed that those nanoparticles were crystalline germanium. Comparing to 
experiment 4, it was believed that the reaction temperature makes the difference on the nucleation of germanium nanoparticles.

The FTIR spectrum of this sample is shown in Figure 5-20. This sample showed a slightly different spectrum from the one of free oleic acid. In the spectrum of free oleic acid, a peak corresponding to $\mathrm{C}=\mathrm{O}$ from the carboxyl group can be observed at around $1711 \mathrm{~cm}^{-1}$. However this peak cannot be seen in the spectrum of the sample of germanium nanoparticles. Since O-H wagging around $1438 \mathrm{~cm}^{-1}$ could not be seen in the spectrum of germanium nanoparticles, oxygen atom from $\mathrm{OH}$ group is attached to the surface of the germanium quantum dots. There is a peak observed at $1650 \mathrm{~cm}^{-1}$ which probably corresponds to $\mathrm{C}=\mathrm{O}$ stretching from the carboxyl group. This peak shift from $1711 \mathrm{~cm}^{-1}$ to $1650 \mathrm{~cm}^{-1}$ is probably due to the attachment of oxygen atoms from the $\mathrm{OH}$ group to germanium atom. The peaks observed at $1547 \mathrm{~cm}^{-1}$ and 1460 $\mathrm{cm}^{-1}$ are probably from $\mathrm{C}-\mathrm{H}$ stretching from the carbon chain next to the carboxyl group.

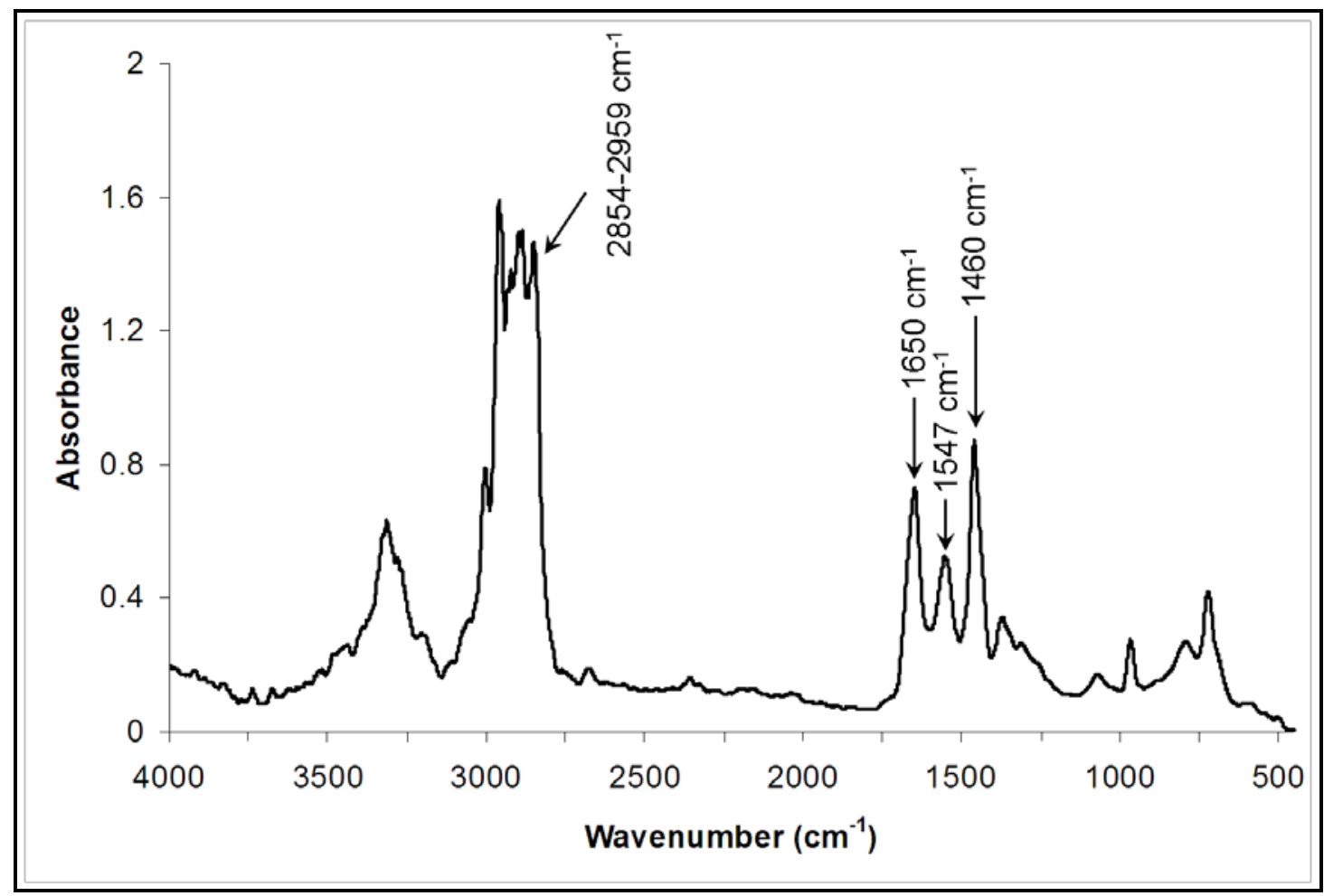

Figure 5-20: FTIR spectrum of germanium quantum dots capped with oleic acid 


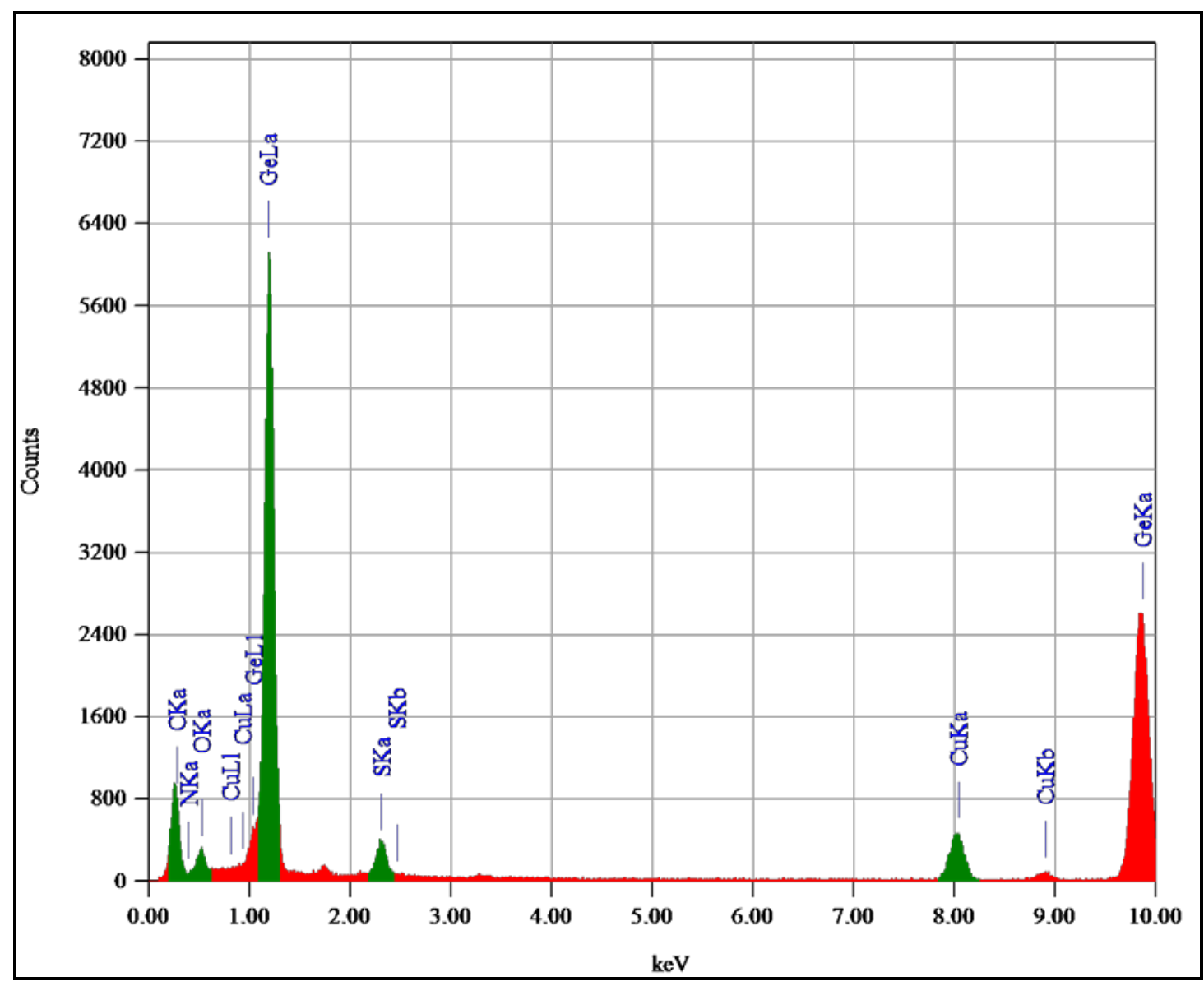

Figure 5-21: EDS spectrum of the sample obtained from experiment 10 


\section{Conclusion and discussion of high temperature bench top syntheses}

Table 5-1: Reaction conditions of the high temperature bench top syntheses; TOA (trioctylamine), HDA (hexadecylamine)

\begin{tabular}{|c|c|c|c|c|c|}
\hline Exp. & Solvent & $\begin{array}{l}\text { Reaction } \\
\text { time (min) }\end{array}$ & $\begin{array}{c}\text { Temperature } \\
\left({ }^{\circ} \mathrm{C}\right)\end{array}$ & $\begin{array}{l}\text { Capping } \\
\text { molecule }\end{array}$ & $\begin{array}{c}\text { Particle } \\
\text { size } \\
\text { (nm) }\end{array}$ \\
\hline 4 & HDA & 40 & 260 & oleic acid & no particles \\
\hline 5 & oleylamine & 20 & 290 & $\begin{array}{c}\text { mercapto } \\
\text { undecanoic } \\
\text { acid }\end{array}$ & $40.5 \pm 5.7$ \\
\hline 6 & oleylamine & 45 & 300 & octadecene & $90.6 \pm 15.7$ \\
\hline 7 & oleylamine & 20 & 290 & folic acid & $41.2 \pm 6.5$ \\
\hline 8 & TOA & 20 & 300 & $\begin{array}{c}\text { 11-dodecen } \\
1-\text { ol }\end{array}$ & $27.1 \pm 5.9$ \\
\hline 9 & oleylamine & 20 & 290 & $\begin{array}{c}10- \\
\text { undecenoate }\end{array}$ & $42.8 \pm 7.5$ \\
\hline 10 & TOA & 15 & 300 & oleic acid & $5.6 \pm 1.3$ \\
\hline
\end{tabular}

Synthesis of high quality germanium nanocrystals from solution-phase technique has been proved challenging because of the strong covalent bond of precursors and high crystallization temperature. The reaction condition of each experiment was summarized in Table 5-1. In Experiments 4 it was confirmed that the synthesis method requires higher than $260{ }^{\circ} \mathrm{C}$ to produce spherical nanoparticles. Since the colour change of the reaction mixture was observed which indicates the dephenylation of germanium precursor this could be due to failure of breaking Ge-S bond from intermediate to form germanium nanocrystals. From the rest of the experiments, relatively mono dispersed nanoparticles were obtained however the average size of the nanoparticles from most of them was larger than the germanium exciton Bohr radius therefore no luminescent nanoparticles were obtained. In terms of the reaction temperature, all the experiments conducted at $290{ }^{\circ} \mathrm{C}$ showed around the similar 
particle sizes even though the different capping molecules were used on each experiment. It suggests that the capping molecules did not affect the nucleation of the germanium nanoparticles. On the other hand, the reactions conducted at $300{ }^{\circ} \mathrm{C}$ showed quite diverse results in particle sizes. It could be due to the solvents and reaction time. Ali-Salim et. al reported the effect of solvent on the CdSeS nanoparticles by using various types of solvent including oleylamine and trioctylamine. ${ }^{27}$ They claimed that oleylamine is a primary amine which has a long carbon chain and can be used as a coordinating solvent. In contrast, trioctylamine (TOA) is a neutral solvent. Therefore the coordinating solvent can slow down the nucleation of the nanoparticles by coordinating the nanoparticles. However in the experiments of 6,8 , and 9 , the reaction conducted in oleylamine showed smaller particle size than the ones in trioctylamine. It is possibly due to the reaction time. The results from experiment 6,8 and 9 showed that the particle size is proportional to the reaction time and it corresponds to the report from Jiang et. al. ${ }^{18}$

From experiments 5 and 7, it was confirmed that when mercapto groups and carboxyl groups exist at both terminal ends of the molecule the sulfur atom from the mercapto group tends to form bonds with germanium rather than the oxygen atoms from the carboxyl group. However in other cases, oxygen atoms from the carboxyl group formed covalent bonds with germanium. The bond energy of Ge-S is $244.32 \mathrm{~kJ} / \mathrm{mol}$ and it is weaker than Ge-O $(447.26 \mathrm{~kJ} / \mathrm{mol})$, therefore the attachment of sulfur atoms onto the surface of the germanium quantum dots could be due to a steric barrier of carboxyl group.

Experiment 9 showed terminal double bonds can also form covalent bonds with germanium. The bond strength of Ge-C is $246.62 \mathrm{~kJ} / \mathrm{mol}$ which is similar to Ge-S bond strength. However from FITR analysis, the evidence of the attachment of $\mathrm{C}=\mathrm{C}$ bond to the germanium nanoparticles was seen. It is probably due to the methoxy group of the capping molecule. Unlike the hydrogen atom of the carboxyl group, the methoxy group of methyl 10-undecenoate cannot form a carboxylate anion which can form a C-O bond. 


\section{5-3. Discussion and Summary}

Two different syntheses systems of germanium nanoparticles showed quite different results. From the experiments 1 and 2, it was confirmed that nanoparticles do not precipitate out but stay in the solution. On the other hand, in all the samples from the high temperature bench top synthesis the nanoparticles precipitated even though their particle sizes were smaller than the ones from experiment 2. The result suggests that the solubility of the nanoparticles is strongly dependent on the surface polarities. Allylamine has a very short carbon chain (three carbons) next to the amine group which makes it water soluble, On the other hand, most of the capping agents used in high temperature bench top synthesis have longer carbon chain which makes the nanoparticles less polar and also it makes them heavier. Therefore these nanoparticles were more likely to precipitate in ethanol during the purification process.

Possible reason for the chloride peak in the EDS analysis of experiment 2 could be due to the remained chlorine on the surface of germanium nanoparticles. Since significant amount of germanium of germanium tetrachloride is lost as germane gas, it is very difficult to calculate the exact amount of reducing agent which needs to be added to remove the chlorine of the germanium tetrachloride. If the amount of reducing agent was not enough to remove all the $\mathrm{Ge}-\mathrm{Cl}$ bonds of the precursor, the germanium nanoparticles remained partially chlorine terminated. Ge-H bond energy (307.65 kJ/mole) is very close to Si-H bond energy (311.83 kJ/mole) therefore it is expected that UV-irradiation at $254 \mathrm{~nm}$ is suitable to break Ge-H bond from hydrogen terminated germanium nanoparticles to form Ge-C bond with the capping agent. ${ }^{28-30}$ However Ge-Cl bond energy is $407.55 \mathrm{~kJ} /$ mole which is higher than Ge-H or Si-H therefore it $254 \mathrm{~nm}$ of UV-irradiation may not be suitable and could not remove chlorine from the surface of the germanium nanoparticles. There are very few reports on the surface modification of the germanium nanoparticles. Warner et. al. and Prabakar et. al reported amine group terminated germanium quantum dots by using platinum catalyst to produce water soluble germanium quantum dots. ${ }^{14,15}$ However, no characterization data of the surface molecules was mentioned. To our best knowledge, there is no report on the surface modification of germanium nanoparticles by UVirradiation therefore the trials of the UV-irradiation capping on germanium are very novel. 
Compared to microemulsion system, high temperature bench top system enabled to gain the germanium nanoparticle as a solid with much narrower size distrubution without size exclusion column chromatography. In addition by using sulfur to promote the formation of germanium nanoparticles, the reaction could be conducted at much lower temperature compared to the thermal decomposition of other precursors such as tetraethylgermane (TEG) or diphenyl germane (DPG) in which the reactions typically performed between 400 to $500{ }^{\circ} \mathrm{C}$. This method enabled to conduct the reaction in standard laboratory fume hood without a dry glove-box. Also it does not require much of expensive chemicals or equipments.

There have been quite a few reports on germanium nanoparticle synthesis however, to our best knowledge, there are very few reports so far about surface characterization of germanium nanoparticles from the high temperature technique. Therefore, the research on investigation of the capping agents to attach on the germanium nanoparticles was very novel.

The possible reasons that SAED could not be obtained from any of the samples are due to the impurities or their smaller size. The nanoparticles obtained from high temperature bench top syntheses were purified only by centrifugation which leaves the possibility of the existence of other heavy impurities in the sample solutions. For the experiment 3 , the sample was purified by size exclusion column chromatography, however, the amount of particles observed in TEM was very small and their sizes were probably too small to give the electron diffractions.

Synthesis systems of germanium quantum dots still need to be improved in ways of size control, surface modification and crystallization to obtain luminescent nanoparticles. The details of the future work will be mentioned in chapter 7 . However surface modification with a variety of functional group in high temperature system is novel and improved the possibility of biological applications of germanium nanoparticles. 


\section{5-4. References}

1. H. W. Chiu, S. M. Kauzlarich; Chem. Mater., 2006, 18, 1023-1028, "Investigation of Reaction Conditions for Optimal Germanium Nanoparticle Production by a Simple Reduction Route”

2. R. S. Tanke, S. M. Kauzlarich, T. E. Patten, K. A. Pettigrew, D. L. Murphy, M. E. Thompson, H. W. H. Lee; Chem. Mater., 2003, 15, 1682-1689, "Synthesis of Germanium Nanoclusters with Irreversibly Attached Functional Groups: Acetals, Alcohols, Esters, and Polymers”

3. B. R. Taylor, S. M. Kauzlarich, H. W. H. Lee, G. R. Delgado; Chem. Mater., 1998, 10, 22-24, “Solution Synthesis of Germanium Nanocrystals Demonstrating Quantum Confinement”

4. B. R. Taylor, S. M. Kauzlarich, H. W. H. Lee, G. R. Delgado; Chem. Mater., 1999, 11, 2493-2500, "Solution Synthesis and Characterization of Quantum Confined Ge Nanoparticles”

5. X. Ma, F. Wu, and S. M. Kauzlarich; J. Solid State Chem., 2008, 181, 16281633, “Alkyl-terminated crystalline Ge nanoparticles prepared from NaGe; Synthesis, functionalization and optical properties”

6. N. Myung, X. Lu, K. P. Johnston, and A. J. Bard; Nano Lett., 2004, 4, 183-185, "Electrogenerated Chemiluminescence of Ge Nanocrystals"

7. X. Lu, K. J. Ziegler, A. Ghezelbash, K. P. Johnston, and B. A. Korgel; Nano Lett., 2004, 4, 969-974, “ Synthesis of Germanium Nanocrystals in High Temperature Supercritical Fluid Solvents”

8. X. Lu, B. A. Korgel, and K. P. Johnston; Chem. Mater., 2005, 17,6479-6485, “ High Yield of Germanium Nanocrystals Synthesized from Germanium Diiodide in Solution” 
9. A. Kornowski, M. Giersig, R. Vogel, A. Chemseddine, and H. Weller; Adv. Mater., 1993, 5, 634-636, "Nanometer-sized colloidal germanium particles: Wet-chemical synthesis, laser-induced crystallization and particle growth”

10. H. Miguez, and V. Fornés; Appl. phys. Lett., 1996, 69, 2347-2349, "Low - temperature synthesis of Ge nanocrystals in zeolite Y"

11. R. H. James; J. Chem. Phys., 1994, 101, 1607-1615, “Germanium quantum dots: Optical properties and synthesis”

12. R. H. James, and K. L. Francoise; Chem. Phys. Lett., 1993, 208, 263-268, “A liquid solution synthesis of single crystal germanium quantum wires”

13. J. P. Wilcoxon, P. P. Provencio, G. A. Samara; Phys. Rev. B, 2001, 64, Art. No. 035417, "Synthesis and optical properties of colloidal germanium nanocrystals”

14. J. H. Warner, R. D. Tilley; Nanotechnol., 2006, 17, 3745-3749, "Synthesis of water-soluble photoluminescent germanium nanocrystals”

15. S. Prabakar, A. Shiohara, S. Hanada, K. Fujioka, K. Yamamoto, R. D. Tilley; Chem. Mater., 2010, 22, 482-486, "Size Controlled Synthesis of Germanium Nanocrystals by Hydride Reducing Agents and Their Biological Applications”

16. N. H. Chou, K. D. Oyler, N. E. Motl, and R. E. Schaak; Chem. Mater., 2009, 21, 4105-4107, “Colloidal Synthesis of Germanium Nanocrystals Using Room-Temperature Benchtop Chemistry”

17. N. Zaitseva, Z. R. Dai, C. D. Grant, J. Harper, and C. Saw; Chem. Mater., 2007, 19, 5174-5178, “Germanium Nanocrystals Synthesized in High-Boiling -Point Organic Solvents” 
18. H. P. Wu, M. Y. Ge, C. W. Yao, Y. W. Wang, Y. W. Zeng, L. N. Wang, G. Q. Zhang, and J. Z. Jiang; Nanotechnol., 2006, 17, 5339-5343, "Blue emission of Ge nanocrystals prepared by thermal decomposition”

19. E. J. Henderson, C. M. Hessel, and J. G. C. Veinot; J. Am. Chem. Soc., 2008, 130, 3624-3632, “Synthesis and Photoluminescent Properties of SizeControlled Germanium Nanocrystals from Phenyl Trichlorogermane-Derived Polymers”

20. J. H. Warner; Nanotechnol., 2006, 17, 5613-5619, “Solution-phase synthesis of germanium nanoclusters using sulphur"

21. http://www.praxair.com/praxair.nsf/AllContent/9ACB6769F6CDBEE7852572 58006F2F39/\$File/p4821e.pdf

22. V. M. Schmidt, and H. Schumann; Zeitschrift für anorganische und allgemeine Chemie, 1963, 325, 130-138, "Spaltungsreaktionen metallorganischer Verbindungen mit Chalkogenen. Reaktionen von Schwefel mit silicium-, germanium- und bleiorganischen Verbindungen”

23. http://en.wikipedia.org/wiki/Oleic_acid

24. C. Jing, X. Zang, W. Bai, J. Chu, and A. Liu; Nanotechnol., 2009, 20, 505607, "Aqueous germanate ion solution promoted synthesis of worm-like crystallized Ge nanostructures under ambient conditions”

25. http://en.wikipedia.org/wiki/Folic_acid

26. S. W. Bailey, and J. E. Ayling; Proc Natl Acad Sci U S A, 2009. 106, 15424 15429, "The extremely slow and variable activity of dihydrofolate reductase in human liver and its implications for high folic acid intake”

27. N. Al-Salim, A. G. Young, R. D. Tilley, A.. J. McQuillan, and J. Xia; Chem. Mater., 2007, 19, 5185-5193, "Synthesis of CdSeS Nanocrystals in 
Coordinating and Noncoordinating Solvents: Solvent's Role in Evolution of the Optical and Structural properties”

28. Z. F. Li, E. Ruckenstein; Nano Lett., 2004, 4, 1463-1467, "Water-Soluble Poly(acrylic acid) Grafted Luminescent Silicon Nanoparticles and Their Use as Fluorescent Biological Staining Labels”

29. S. Sato, M. T. Swihart; Chem. Mater., 2006, 18, 4083-4088, "Propionic-AcidTerminated Silicon Nanoparticles: Synthesis and Optical Characterization”

30. H-Ch. Weissker, J. Furthmu“ller, and F. Bechstedt; Phys. Rev. B., 2003, 67, 245304, "Structural relaxation in Si and Ge nanocrystallites: Influence on the electronic and optical Properties” 


\section{Chapter 6 Synthesis and Characterisation of Silica nanoparticles for dry eye diagnosis}

This chapter describes the synthesis of silica nanoparticles encapsulating organic dyes to obtain fluorescence for dry eye diagnosis. The silicon quantum dots synthesised in the previous chapters have a photoluminescent peak (430 nm) and an excitation wavelength $(320 \mathrm{~nm})$ that are too high in energy for use in human eyes. The motivation of this research was to produce non-toxic nanoparticles which can be excited with a lower energy to create fluorescence. Presently organic dyes such as fluorescein or rose Bengal are used for dry eye testing. ${ }^{1}$ As mentioned in chapter 1 , there are three layers in the tear films and the interactions of each layer plays a critical role in protecting the eyes. ${ }^{2-9}$ To advance the research in this field, it is necessary to find out more details of the layer interactions. However there has been a limit in the applications of organic dyes in studying these tear film layers. The emission life time of the organic dyes is short compared to quantum dots. It is also difficult to use multiple dyes to mark different layers of tear film at the same time because of the difficulties to change their polarity. Most of the organic dyes being used in eye research are soluble in polar solvents, therefore it is difficult to use them as a lipid layer marker of the tear film. Therefore it has been difficult to apply multiple dyes as tear film markers at the same time.

Because of their photo-stability and unique optical properties, quantum dots are potential candidates as a tear film marker in dry eye research applications. Due to safety reasons, CdSe quantum dots are not suitable for use in human eyes even though they exhibit excellent optical properties. ${ }^{10}$ In terms of safety, the silicon quantum dots synthesised in previous chapters were considered as potential candidates. However they could not succeed in the application because of their low quantum yield. In addition they cannot provide multiple fluorescence and require a relatively high energy to excite them to fluoresce. This is a problem for dry eye diagnosis as short wavelength ultra-violet light can cause damage to eyes Therefore silica nanoparticles encapsulating organic dyes have the potential as a good candidate for a new tool in dry eye diagnosis. 
There have already been a few reports published regarding the synthesis of silica nanoparticles encapsulating organic dyes. ${ }^{11-17}$ As mentioned in the introduction chapter Mohr et. al. reported rhodamine doped silica nanoparticles using silylated sulforhodamine derivative ${ }^{18}$ and tris (2,2'-bipyridine) dichlororuthenium (II) hexahydrate (Rubpy) doped silica nanoparticles were reported by Tan et. al. ${ }^{19}$ However to the best of our knowledge, there is no application of silica nanoparticles in dry eye diagnosis research. Therefore the trials of organic dye doped silica nanoparticles in this chapter are conducted for the purpose of this application. One of the main adventages of encapsulating these organic dyes in the silica nanoparticles is that the polarity can be changed by modifying the surface of the silica nanoparticles so that they can be used as markers for different tear layers. Also by encapsulating the organic dyes the life time of photoluminescence can be enhanced. ${ }^{13}$

Rhodamine and fluorescein were chosen as organic dyes and all the samples were characterised by SEM.

\section{6-1. Synthesis of organic dye doped silica nanoparticles}

\section{6-1-1. Rhodamine doped silica nanoparticles (Experiment 1 and 2)}

For the first trial, rhodamine was chosen as the organic dye since it has close to $100 \%$ quantum yield and a fluorescence emission around $580-600 \mathrm{~nm}$ which is suitable as a film marker. Since it was difficult to purchase rhodamine derivative compounds because of the price and the delivery time, the free rhodamine molecule (Figure 6-1) was tested on very simple synthesis methods.

Two methods were used to synthesise the rhodamine conjugated silica nanoparticles, as described below. 


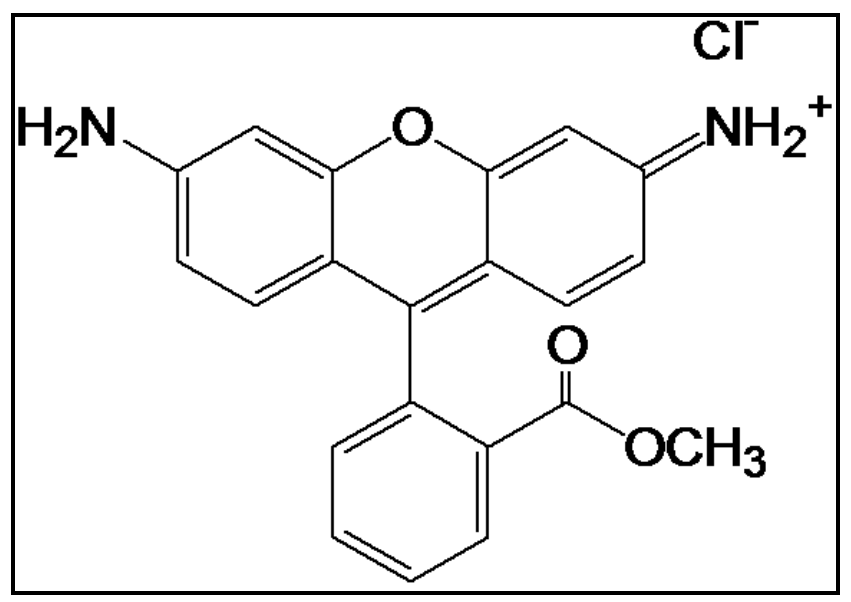

Figure 6-1: a molecular structure of rhodamine

\section{Experiment 1}

Using the main principle of sol-gel method (Stober method) which has been widely exploited since 1968 silica nanoparticles were synthesised in alcohol solvent, Rhodamine was added to the reaction mixture to conjugate with the silica nanoparticles. The positively charged nitrogen atom of rhodamine dye is expected to form electrostatic bond between the lone pair electrons of the oxygen atom of the surface of the silica nanoparticles.

\section{Experimental}

In a round bottom flask, $1.45 \mathrm{~mL}$ of Mili Q water, $1.38 \mathrm{~mL}$ of $35 \%$ ammonia solution and $32.17 \mathrm{~mL}$ of ethanol were mixed and stirred at room temperature. Mixture of $0.336 \mathrm{~mL}$ of tetraethoxysilane (TEOS) and $4.664 \mathrm{~mL}$ of ethanol was slowly added to the solution and then $100 \mu \mathrm{L}$ of $0.1 \mathrm{M}$ rhodamine solution was added dropwise. The solution was stirred for 24 hours at room temperature. ${ }^{20}$

The solutions were white in colour and cloudy, and gave strong yellow fluorescence from rhodamine when illuminated by ultra-violet light.

\section{Purification}

The white precipitate was collected by centrifugation at $14000 \mathrm{rpm}$ for 30 minutes and washed with absolute ethanol for 4 times. 


\section{Result}

The sample was characterised by Scanning Electron Microscopy (SEM). As can be seen in Figure 6-2, mono dispersed nanoparticles were obtained. The average size of the silica nanoparticles was $78 \pm 7 \mathrm{~nm}$. However no fluorescence was observed from the nanoparticles under ultra-violet light. It could be due to the loss of rhodamine dye during the purification process since the electrostatic bond is very weak and the dye may came off from the surface of the silica nanoparticles.

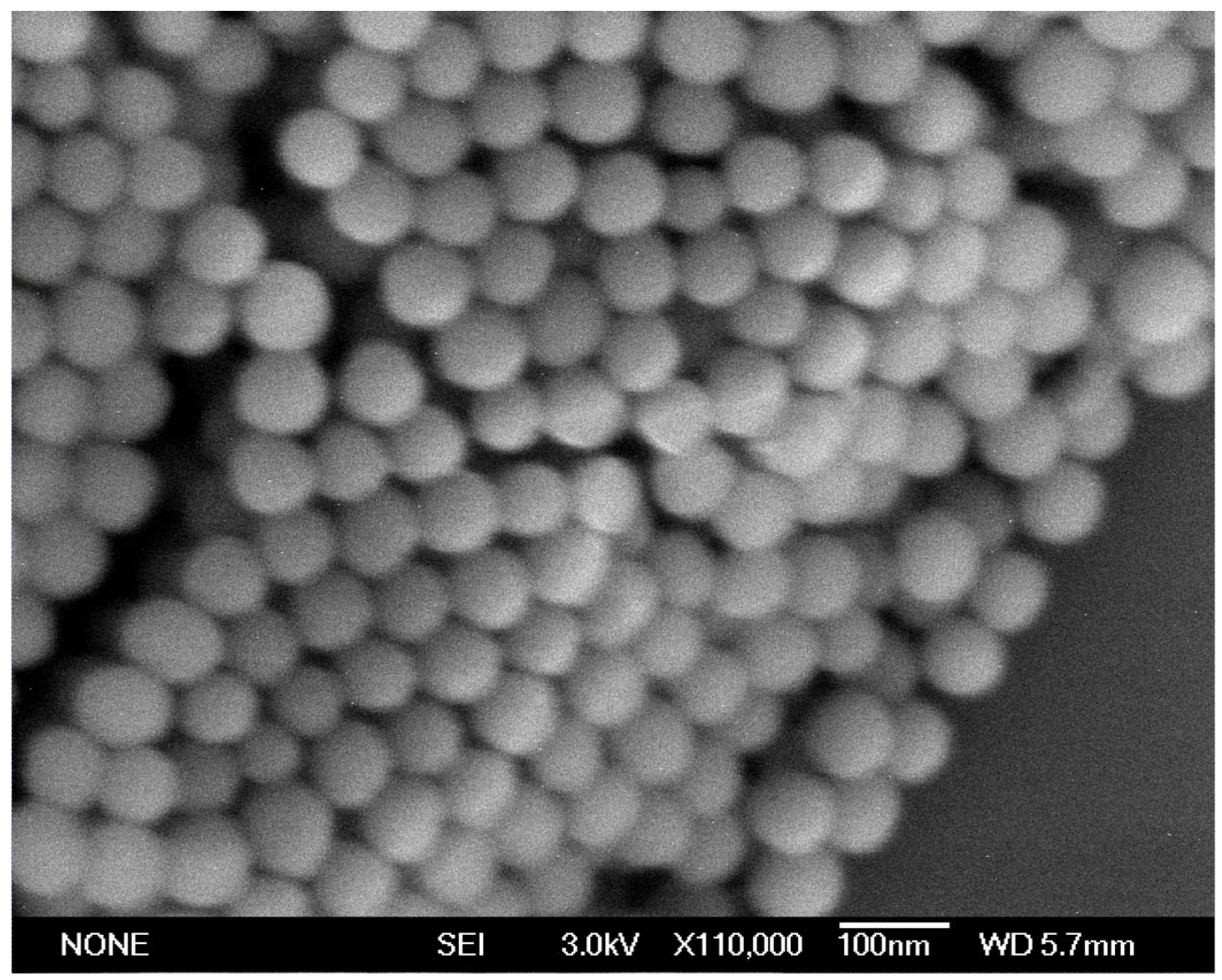

Figure 6-2: An SEM micrograph of silica nanoparticles obtained from experiment 1

\section{Experiment 2}

Followed by sol-gel system, silica nanoparticles were synthesised by water-in-oil microemulsion system. In this system polyoxyethylene octyl phenyl ether (triton X100) was used as a surfactant and particle size and shape were compared to the sample obtained from experiment 1 . Rhodamin was added to the reaction mixture to conjugate the silica nanoparticles. 


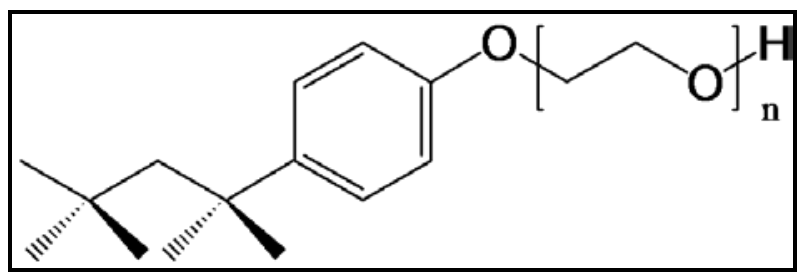

Figure 6-3: A structure of polyoxyethylene octyl phenyl ether (triton X-100)

\section{Experimental}

$1.77 \mathrm{~g}$ of polyoxyethylene octyl phenyl ether (triton X-100), $7.5 \mathrm{~mL}$ of cyclohexane and $1.6 \mathrm{~mL}$ of butanol were mixed in a round bottom flask and mixture of $100 \mu \mathrm{L}$ of tetraethoxysilane (TEOS) and $60 \mu \mathrm{L}$ 35\% ammonia solution was added slowly. 100 $\mu \mathrm{L}$ of $0.1 \mathrm{M}$ rhodamine solution in water was added dropwise and the solution was stirred for 24 hours at room temperature. ${ }^{11}$

The solutions were white in colour and cloudy, and gave strong yellow fluorescence from rhodamine when illuminated by ultra-violet light.

Purification

The white precipitate was collected by centrifugation at 14000 rpm for 30 minutes and washed with absolute ethanol for 4 times.

\section{Result}

Figure 6-4 show the SEM images of the samples from experiments 2. As can be seen in Figure 6-4, spherical, mono dispersed nanoparticles were obtained. The average particle size of the silica nanoparticles was $98 \pm 4 \mathrm{~nm}$. No fluorescence was observed from the sample after purification. 


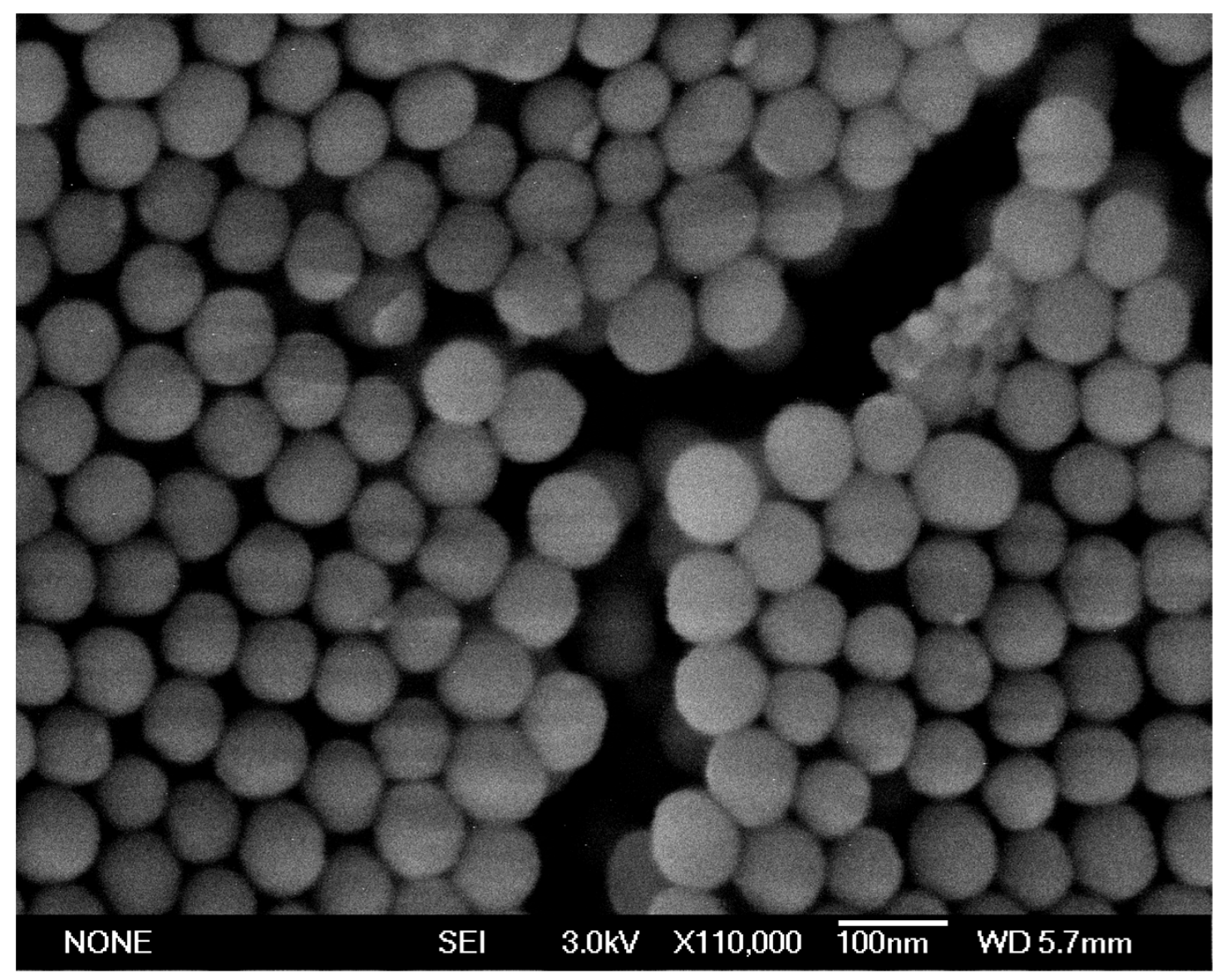

Figure 6-4: An SEM micrograph of silica nanoparticles obtained from experiment 2

As no fluorescence was observed from either sample under ultra-violet light, it was indicated that rhodamine was not properly doped with silica nanoparticles in both experiments even though the nanoparticles were successfully synthesised. This could be due to the dye leaking. Tan et. al. reported that tris(2,2 ${ }^{4}-$ bipyridyl)dichlororuthenium(II) hexahydrate (Rubpy) binds to silica nanoparticles through electrostatic interactions.$^{21}$ As tris(2,2'-bipyridyl)dichlororuthenium(II) hexahydrate (Rubpy), rhodamine has positive charge, however the electrostatic force is weaker compared to covalent bonding. Since tetraethoxysilane (TEOS) cannot bind to rhodamine covalently the dye leaked out of the silica pores during precipitation process. The details will be discussed in a later section.

About the particle size, the microemulsion system showed smaller particle size distribution compared to sol-gel method. It is possibly because in sol-gel method, when the nanoparticles are smaller than $100 \mathrm{~nm}$, the hydration repulsion caused by adsorbing water molecules onto the surface of the nanoparticles becomes very small. Also the potential barrier from the electrostatic repulsion becomes smaller therefore it 
is difficult to keep the dispersion stabilised. On the other hand in the microemulsion system the nanoparticles were produced inside the micelles which are very uniform and have high dispersion stabilisation. ${ }^{22,23}$

\section{6-1-2. Fluorescein doped silica nanoparticles (Experiment 3, 4 and 5)}

Fluorescein has been widely used in eye research for measuring a tear breakup time (TBUT). ${ }^{24}$ Fluorescein is known to be non-toxic and relatively safe compared to other organic dyes for biological use. ${ }^{25}$ The molecular structure is shown in Figure 6-5. The free fluorescein molecule and a derivative compound, fluorescein-5-isothiocyanate (FITC) were used in this study. The same synthesis method as Experiment 1 and 2 was used for fluorescein doped silica nanoparticles to investigate bonding possibility between fluorescein and silica matrix. For the fluorescein-5-isothiocyanate (FITC) molecule, 3-amino-propyltriethoxylsilane (APTES) was used to form a bond between the isothiocyanate group of FITC and the amine group of 3-aminopropyltriethoxylsilane (APTES). All the samples were characterised by SEM.

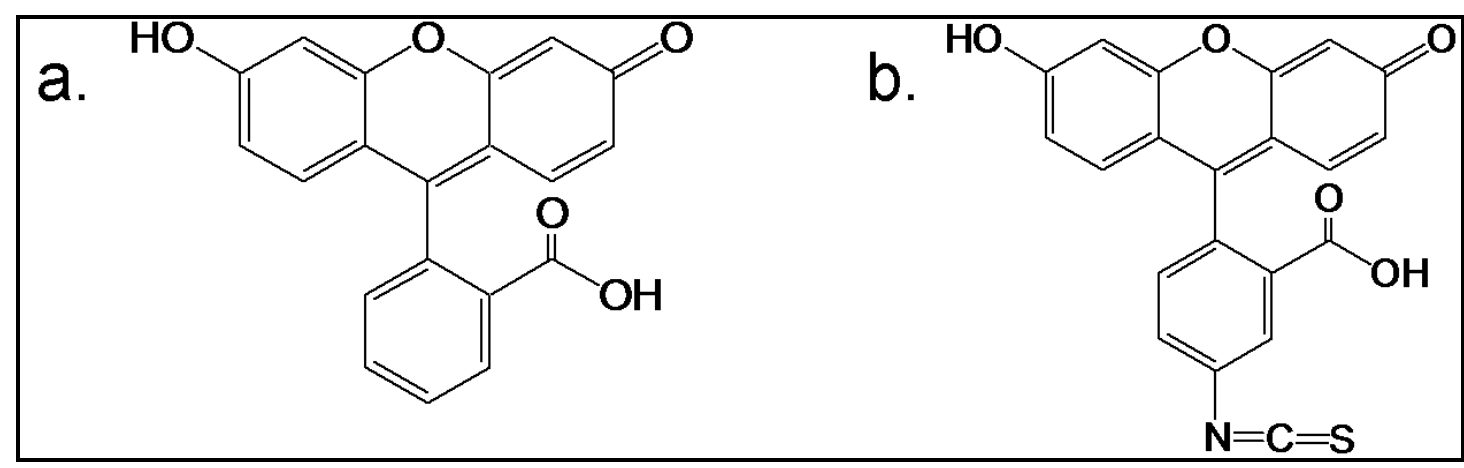

Figure 6-5: Molecular structure of (a); fluorescein, and (b); fluorescein-5isothiocyanate (FITC)

\section{Experiment 3}

Sol-gel synthesis method was applied to synthesise silica nanoparticles conjugated with fluorescein. The Rhodamine conjugation with the silica nanoparticles synthesised by this system did not show fluorescence. The conjugation of fluorescein to silica nanoparticles was investigated by the same synthesis method. 


\section{Experimental}

In a round bottom flask, $1.45 \mathrm{~mL}$ of Mili $\mathrm{Q}$ water and $32.17 \mathrm{~mL}$ of ethanol were mixed and stirred at room temperature. Mixture of $0.336 \mathrm{~mL}$ of tetraethoxysilane (TEOS) and $4.664 \mathrm{~mL}$ of ethanol was added slowly and then $100 \mu \mathrm{L}$ of $0.1 \mathrm{M}$ fluorescein solution was added dropwise followed by slow addition of $1.38 \mathrm{~mL}$ of $35 \%$ ammonia solution to the reaction mixture. The solution was stirred for 24 hours at room temperature.

The solutions were white in colour and cloudy, and fluoresced strongly in greenyellow under ultra-violet light.

Purification

The white precipitate was collected by centrifugation at $14000 \mathrm{rpm}$ for 30 minutes and washed with absolute ethanol for 4 times.

\section{Result}

As can be seen in Figure 6-6, spherical, mono dispersed silica nanoparticles were observed from the samples obtained from experiments 3. The average particle size from experiment 3 was $128 \pm 19 \mathrm{~nm}$. Photoluminescence from fluorescein could not be observed from the sample. It is probably due to the same reason as the experiment 1 and 2. 


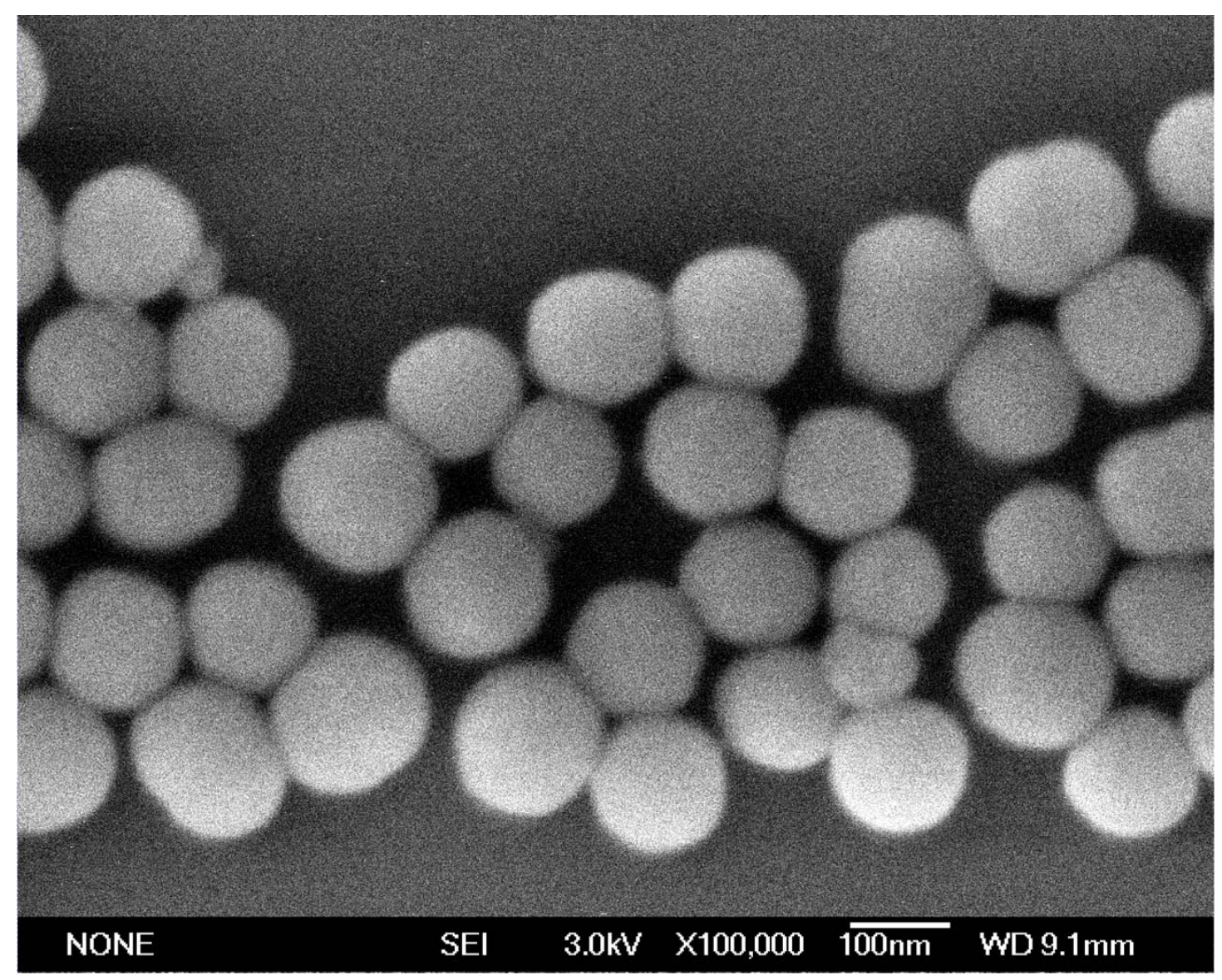

Figure 6-6: A SEM micrograph of silica nanoparticles obtained from experiment 3.

\section{Experiment 4}

As tried with rhodamine, water-in-oil microemulsion synthesis was applied to produce fluorescein conjugated silica nanoparticles. As experiment 2, triton-X was used as surfactant and fluorescein was added to the reaction mixture.

\section{Experimental}

$1.77 \mathrm{~g}$ of polyoxyethylene octyl phenyl ether (triton X-100), $7.5 \mathrm{~mL}$ of cyclohexane and $1.6 \mathrm{~mL}$ of butanol were mixed in a round bottom flask and mixture of $100 \mu \mathrm{L}$ of

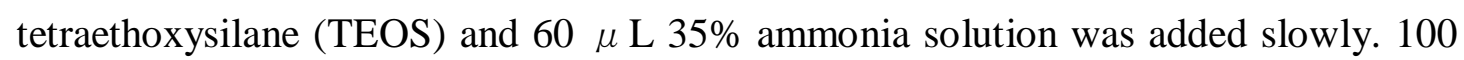
$\mu \mathrm{L}$ of $0.1 \mathrm{M}$ fluorescein solution in water was added dropwise and the solution was stirred for 24 hours at room temperature.

The solutions were white in colour and cloudy, and fluoresce strongly in green-yellow under ultra-violet light. 


\section{Purification}

The white precipitate was collected by centrifugation at $14000 \mathrm{rpm}$ for 30 minutes and washed with absolute ethanol for 4 times.

\section{Result}

As can be seen in Figure 6-7, spherical, mono dispersed silica nanoparticles were observed from the samples obtained from experiment 4 . The average particle size was $93 \pm 7 \mathrm{~nm}$. No photoluminescence from fluorescein could be observed from the sample.

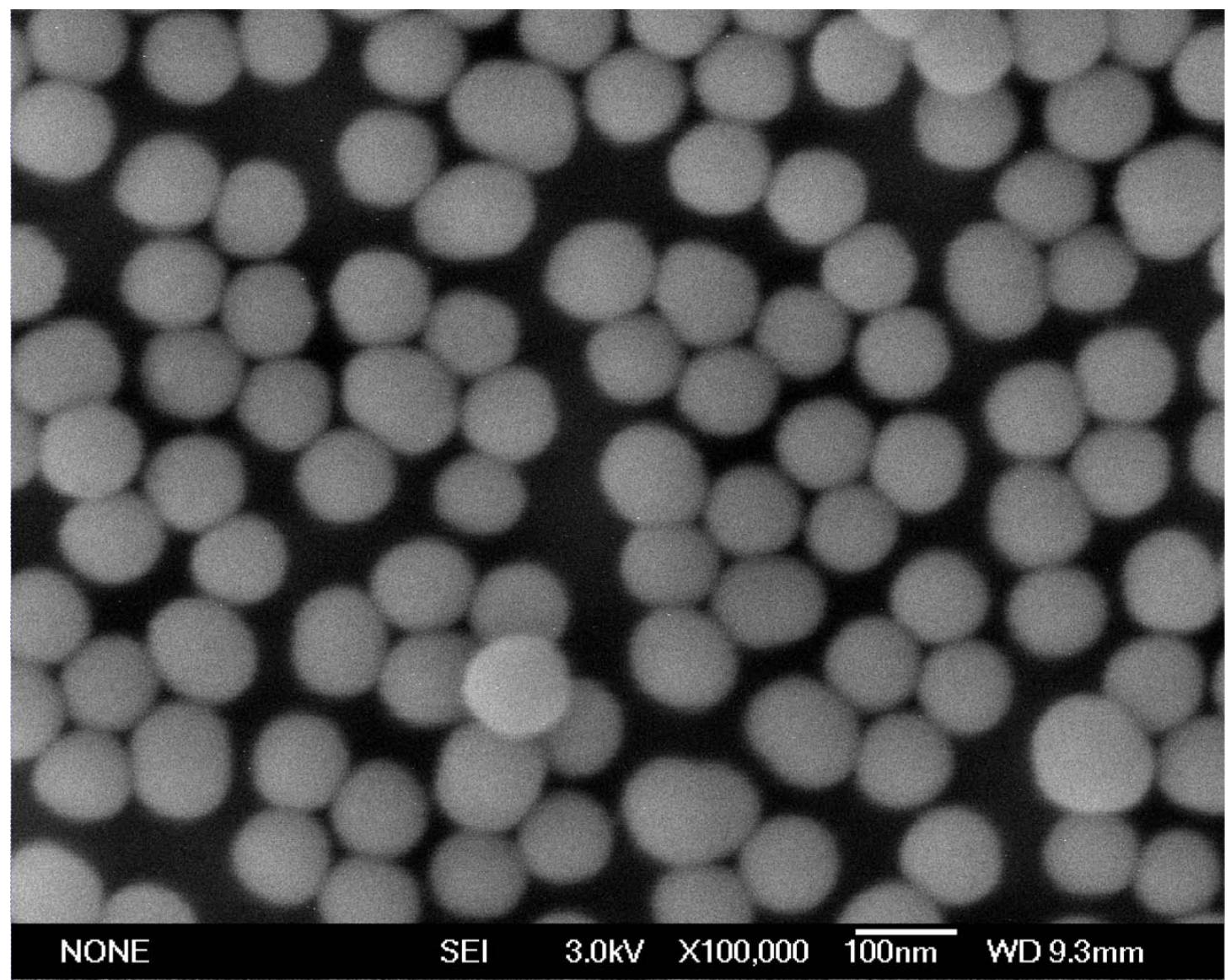

Figure 6-7: A SEM micrograph of silica nanoparticles obtained from experiment 4.

As for the results from experiment 1 and 2, the water-in-oil microemulsion synthesis showed narrower nanoparticle size distribution which can be supported by the theory from Mou et. al. ${ }^{22}$

Both sample from experiment 3 and 4 did not show the evidence to conjugation between fluorescein and the silica nanoparticles. 


\section{Experiment 5}

Since both the sol-gel and water-in-oil microemulsion system used did not produce dye-conjugated silica nanoparticles, a new strategy was used. A linker molecule was applied to form covalent bond between the dye molecule and the nanoparticles. 3amino-propyltriethoxylsilane (APTES) was chosen as the linker to form a covalent bond between the amine group and the isothiocyanate group of the fluorecein derivative (fluorescein-5-isothiocyanate). The synthesis was carried out in two steps. First, fluorescein-5-isothiocyanate was covalently linked with 3-aminopropyltriethoxylsilane (APTES) to form a dye-silane conjugate. Second, the dyesilane conjugate was reacted with the main silica building material tetraethoxysilane (TEOS) to realize the encapsulation.
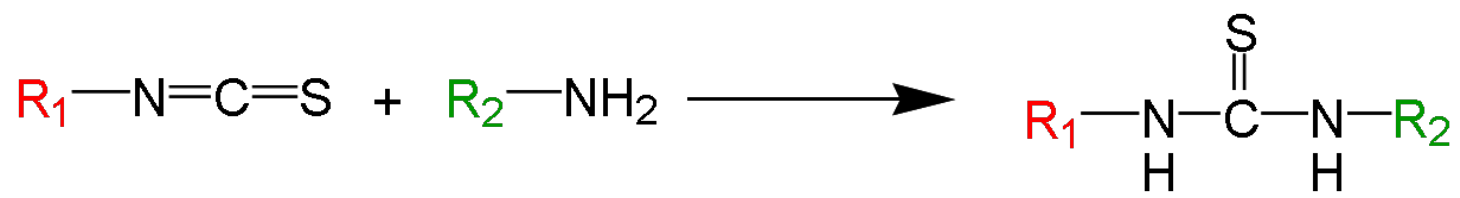

Scheme 6-1-1: Reaction scheme of the isothiocyanate group and amine group

\section{Experimental}

To form the covalent linkage, $100 \mu \mathrm{L}$ of $0.1 \mathrm{M}$ fluorescein-5-isothiocyanate in water was mixed with $23.4 \mu \mathrm{L}(100 \mu \mathrm{mol})$ of 3-amino-propyltriethoxylsilane (APTES) in a round bottom flask. The mixture was stirred overnight in the dark and used immediately the next day. In order to achieve complete encapsulation, $50 \mu \mathrm{L}$ of the solution of APTES/fluorescein-5-isothiocyanate conjugate was mixed with $17 \mathrm{~mL}$ of anhydrous ethanol and $1.28 \mathrm{~mL}$ of 35\% ammonia solution. After stirring for $15 \mathrm{~min}$, $20 \mu \mathrm{L}$ of tetraethoxysilane (TEOS) was added and the mixture was stirred for $18 \mathrm{~h}$.

The solutions were white in colour and cloudy, and fluoresced strongly in greenyellow under ultra-violet light.

\section{Purification}

The white precipitate was collected by centrifugation at $14000 \mathrm{rpm}$ for 30 minutes and washed with absolute ethanol for 4 times. 
Measurement

UV-Vis absorption spectrum of free fluorescein-5-isothiocyanate (FITC) and PL spectra of fluorecein doped silica nanoparticles and free fluorescein-5-isothiocyanate (FITC) were measured for the diluted solution of each sample in ethanol.

For photo-bleaching measurements, $20 \mu \mathrm{L}$ of fluorescein-5-isothiocyanate (FITC) conjugated silica nanoparticles in ethanol was placed on slide glass and fluorescence was observed under optical microscope. Photos were taken every 5 minutes up to 30 minutes. Same procedure was applied to measure the photo-stability of free fluorescein-5-isothiocyanate (FITC).

FTIR analysis was carried out using a Perkin-Elmer spectrometer. $\mathrm{KBr}$ powder was ground into a fine powder and two drops of the silica nanoparticles conjugated with fluorescein-5-isothiocyanate (FITC) in ethanol were added and dried in the oven at $110{ }^{\circ} \mathrm{C}$ for over an hour.

\section{Results}

\section{SEM and DLS}

A SEM micrograph of the sample obtained from experiment 5 is shown in Figure 6-8. Again, spherical mono dispersed silica nanoparticles were obtained, with an average size of $168.5 \mathrm{~nm}$. Size distribution of the sample was measured using dynamic light scattering (DLS). The SEM results are shown in Figure 6-8. As can be seen in the graph the particle size distribution is relatively small with an average size of $163.7 \pm$ $35 \mathrm{~nm}$. This size is slightly larger than the sizes can be observed in SEM micrograph. 


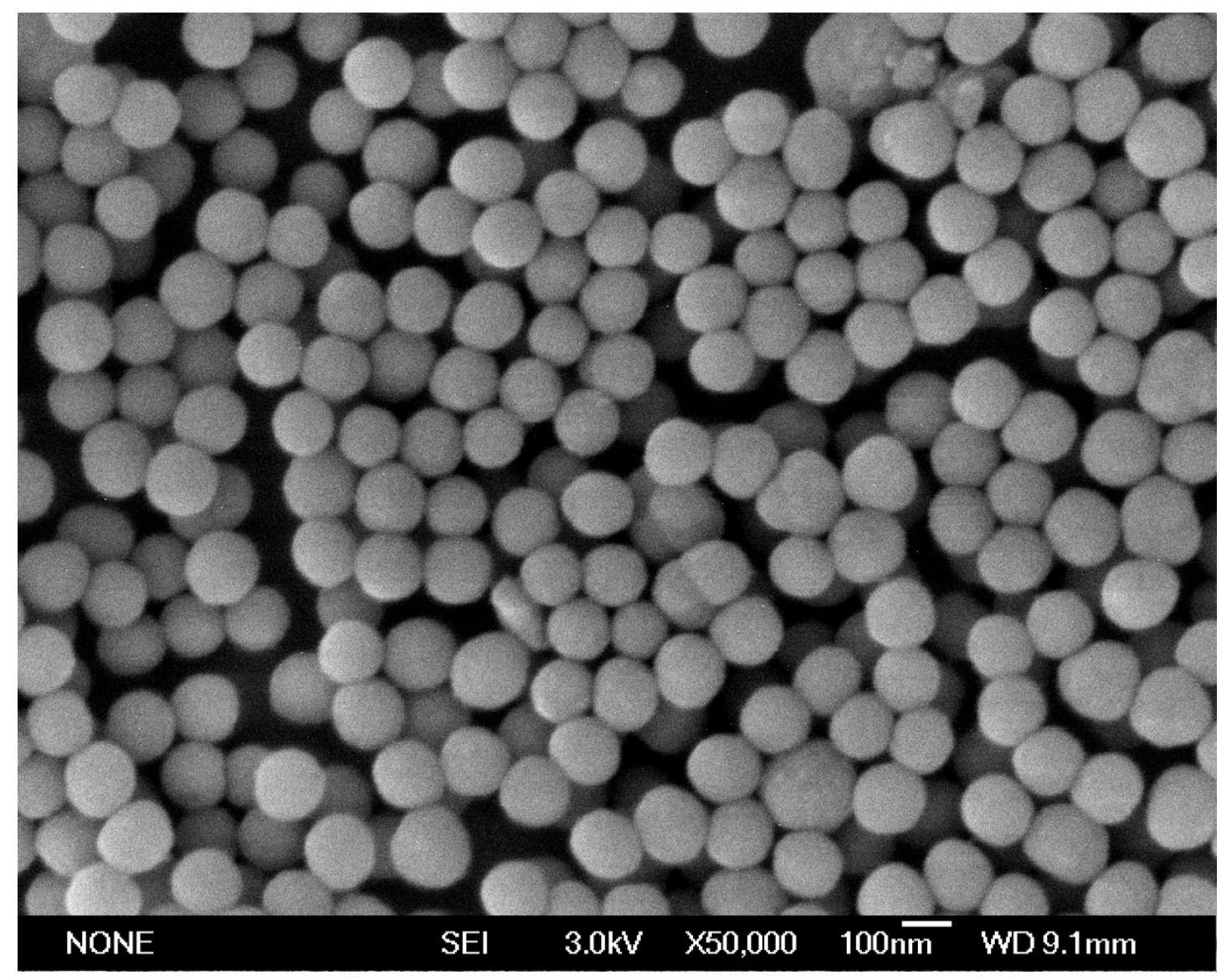

Figure 6-8: A SEM micrograph of the silica nanoparticle conjugated with fluorescein-5-isothiocyanate (FITC) 


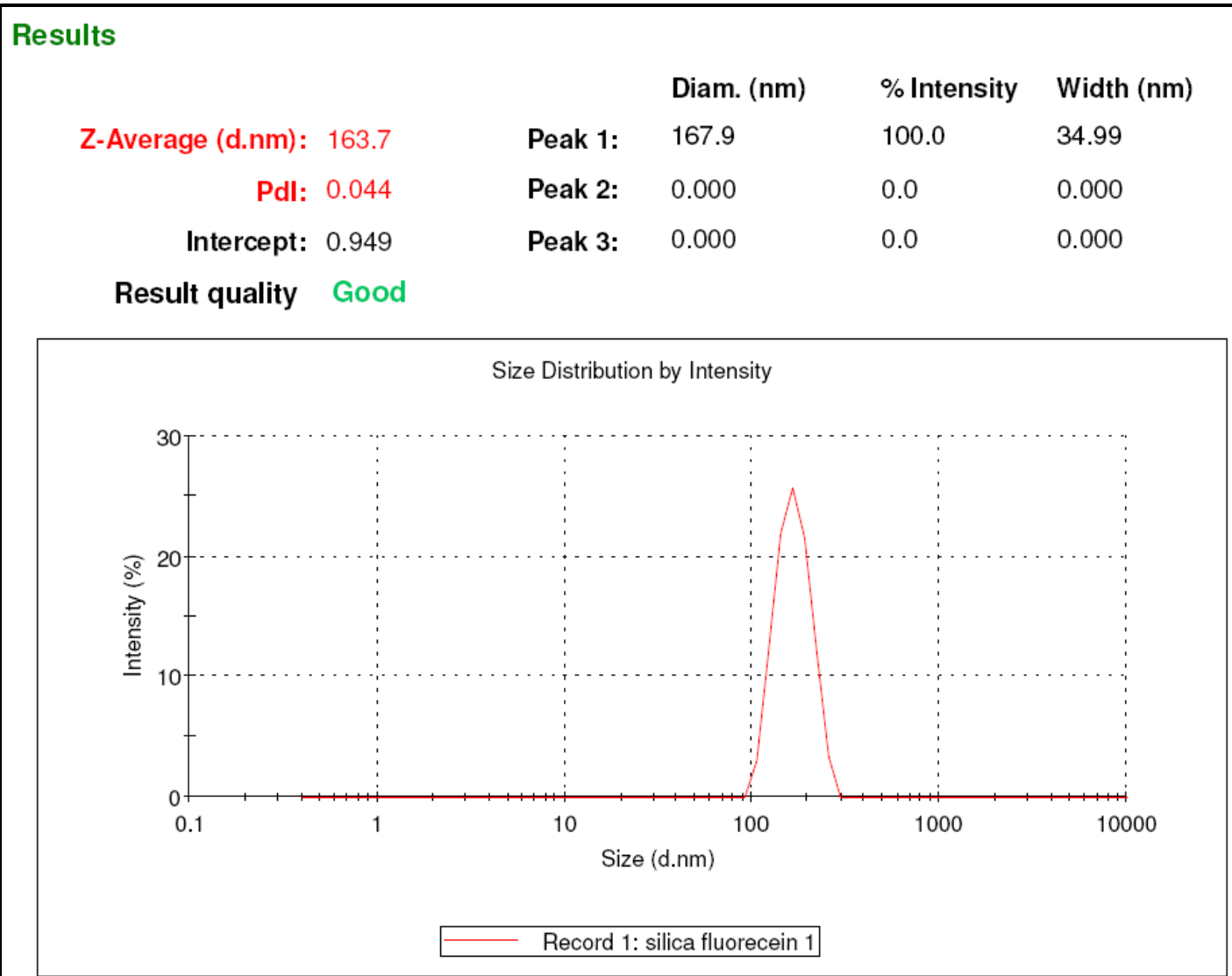

Figure 6-9: Size distribution of silica nanoparticles conjugated with fluorescein-5isothiocyanate (FITC)

\section{Optical properties}

The sample obtained from experiment 5 has strong yellow luminescence under ultraviolet light which indicates that fluorescein-5-isothiocyanate (FITC) has been conjugated with silica precursor and encapsulated in the nanoparticles. The absorption spectrum of fluorescein-5-isothiocyanate (FITC) is shown in Figure 6-10. 


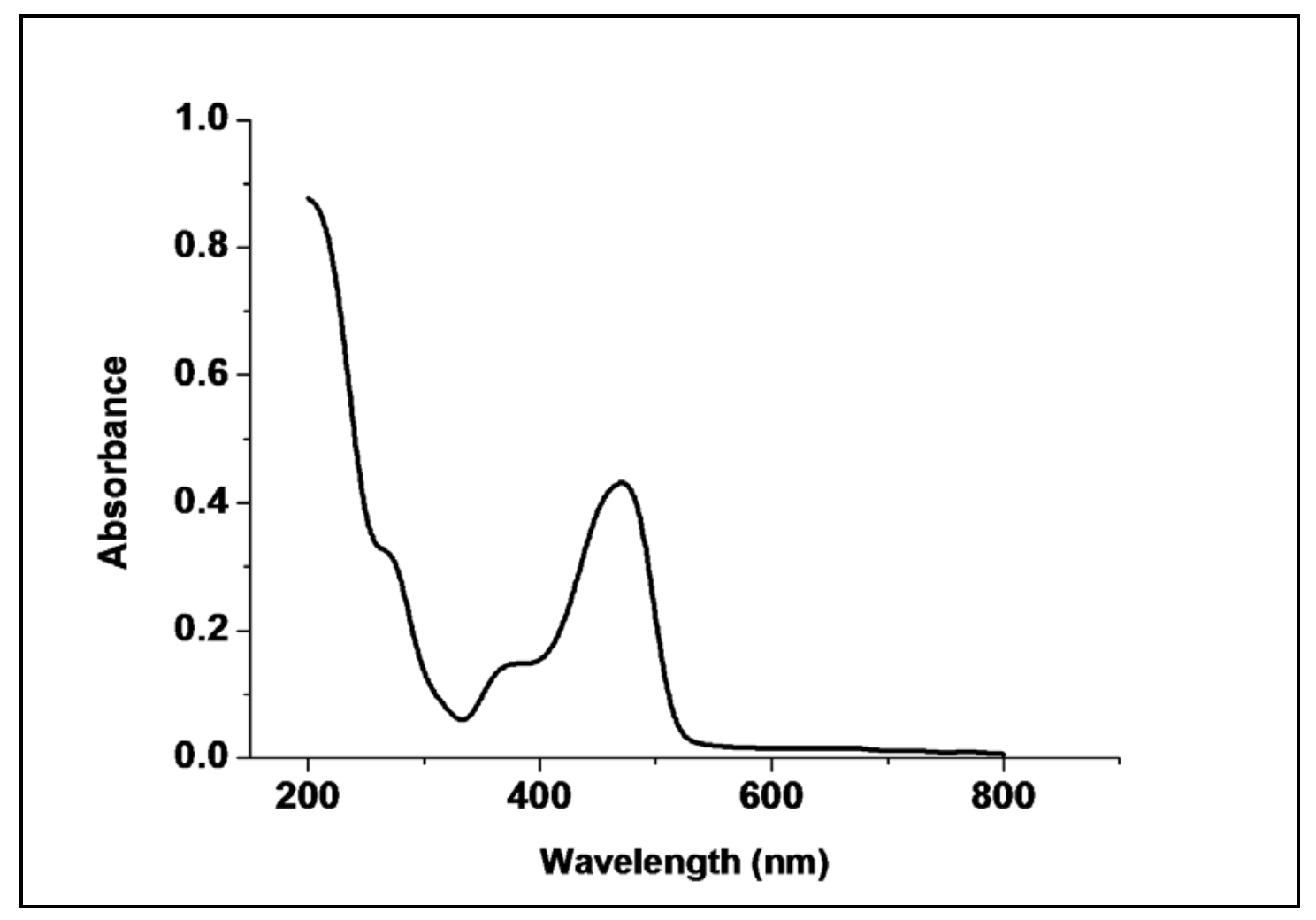

Figure 6-10: Absorption spectra of free fluorescein-5-isothiocyanate

As can be seen in Figure 6-10, free fluorescein-5-isothiocyanate (FITC) has main absorption peak at around $480 \mathrm{~nm}$ and it has a small shoulder just below $400 \mathrm{~nm}$. Absorption spectrum of fluorescein-5-isothiocyanate (FITC) conjugated with silica nanoparticles could not be obtained. It could be due to too much scattering of the excitation wavelength because the solution was very cloudy.

Photoluminescence and excitation spectrum of this sample were also measured. Photoluminescence spectra of the sample and free fluorescein-5-isothiocyanate (FITC) were shown in Figure 6-11 (a) and (b) respectively. 

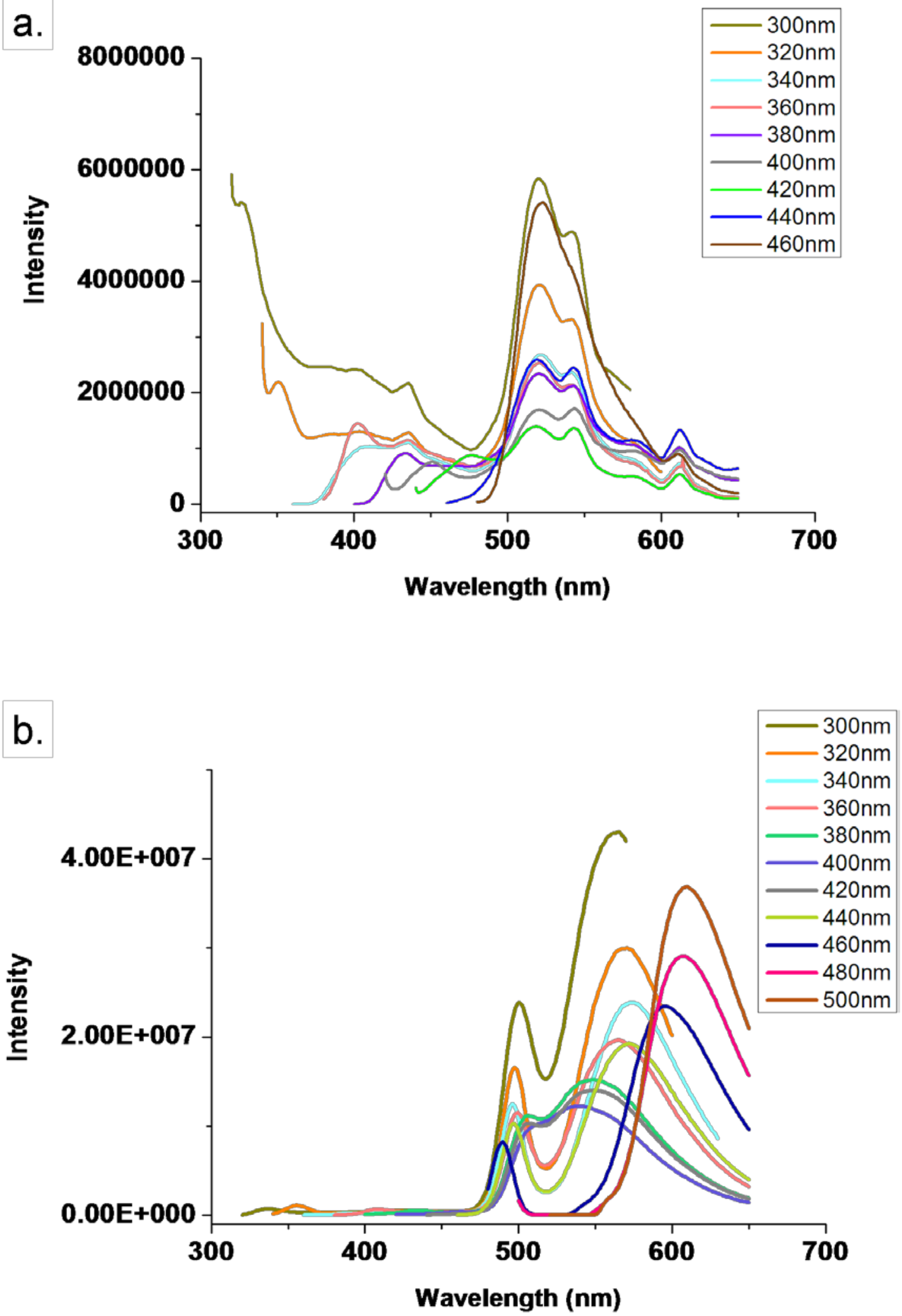

Figure 6-11: (a); Photoluminescence spectra of fluorescein-5-isothiocyanate (FITC) conjugated with silica nanoparticles and (b); free fluorescein-5-isothiocyanate (FITC) 
As may be seen in the Figure 6-11, fluorescein-5-isothiocyanate (FITC) encapsulated in silica nanoparticle and free fluorescein-5-isothiocyanate (FITC) showed quite different emission peaks. The emission peak of fluorescein-5-isothiocyanate (FITC) encapsulated in silica nanoparticle was around $530 \mathrm{~nm}$ and had a small shoulder at around $550 \mathrm{~nm}$. Also the peaks of emission did not shift depending on excitation wavelength. Compared to this sample, photoluminescence spectrum of free fluorescein-5-isothiocyanate (FITC) showed two emission peaks around $480 \mathrm{~nm}$ to $500 \mathrm{~nm}$ and $550 \mathrm{~nm}$ to $620 \mathrm{~nm}$. The similar spectra of free fluorescein-5isothiocyanate (FITC) was seen in the literature. ${ }^{28} \mathrm{~A}$ wider shift of emission peaks was observed depending on excitation wavelength. The position of emission peak of fluorescein-5-isothiocyanate (FITC) encapsulated in silica nanoparticle was around the middle of the two of the emission peaks of free fluorescein-5-isothiocyanate (FITC). The reason for this observation could be due to the bond form between isothiocyanate group from fluorescein-5-isothiocyanate (FITC) and amine group from 3-amino-propyltriethoxylsilane (APTES).

Excitation spectra were also measured for both of the compounds. Figure 6-12 (a) shows the excitation spectrum of fluorescein-5-isothiocyanate (FITC) encapsulated silica nanoparticle and (b) shows the one from free fluorescein-5-isothiocyanate (FITC). 

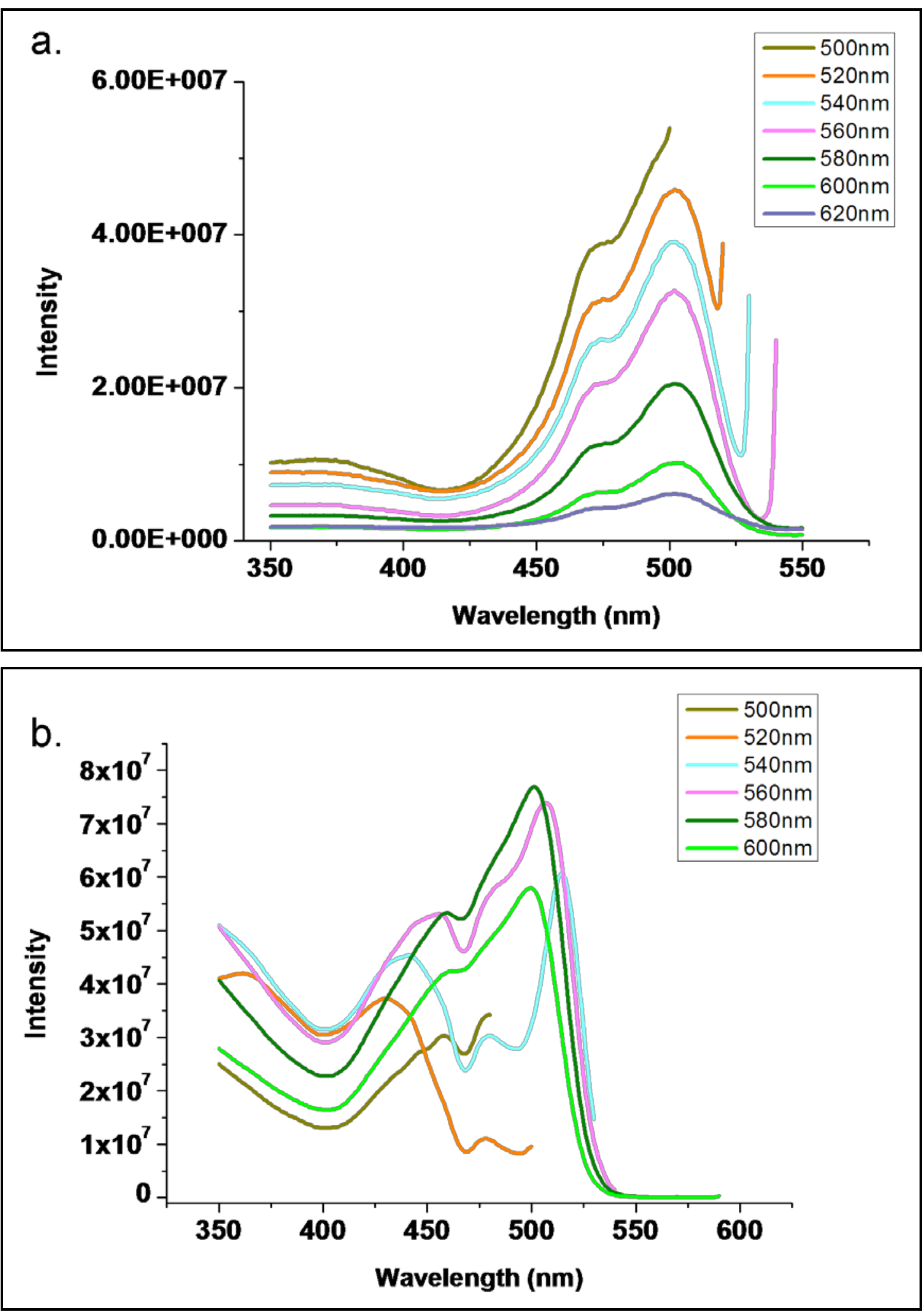

Figure 6-12: Fluorescence excitation spectra of (a); fluorescein-5-isothiocyanate (FITC) encapsulated in silica nanoparticles and (b); free fluorescein-5-isothiocyanate (FITC) 
For free fluorescein-5-isothiocyanate (FITC), fluorescence excitation spectra showed small peaks around $430 \mathrm{~nm}$ to $450 \mathrm{~nm}$ and also the large second peaks around $500 \mathrm{~nm}$. The main large peaks are from the first excited state of $\pi-\pi^{*}$ transition and the small shoulder peaks are from the second excited state which lost more energy in internal conversion. ${ }^{29}$ Very similar result was seen in the fluorescence excitation spectra of fluorescein-5-isothiocyanate (FITC) encapsulated in silica nanoparticles. ${ }^{30}$ The results indicate that isothiocyanate group did not contribute to the optical properties. In the spectra of free fluorescein-5-isothiocyanate (FITC), the first of the two peaks were clearer in $540 \mathrm{~nm}$ and $560 \mathrm{~nm}$ compared to $580 \mathrm{~nm}$ and $600 \mathrm{~nm}$. It indicates that the contribution of the second excited state to the photoluminescence of higher energy monitoring wavelengths was larger than that of the lower energy monitoring wavelengths.

\section{Photo-stability}

Photo-stability of the silica nanoparticles conjugated with fluorescein-5isothiocyanate (FITC) was compared to free fluorescein-5-isothiocyanate (FITC) molecules. The photo-stability was measured under optical microscopy excited by blue light (360-400 nm) and images were taken every 5 minutes to see the photobleaching. The results were shown in Figure 6-13. 


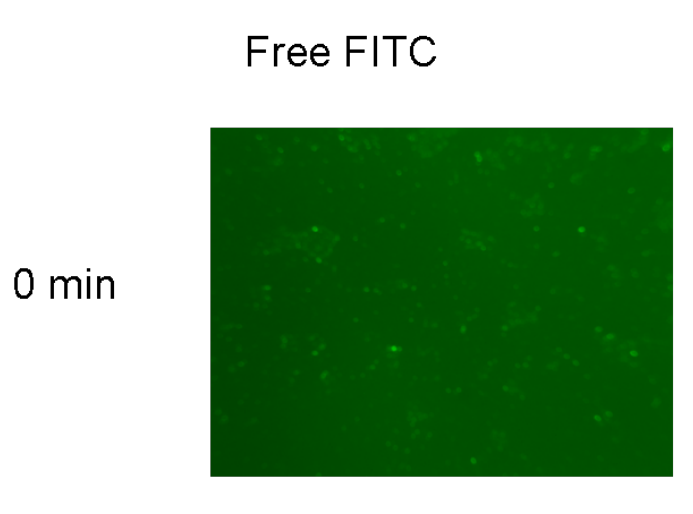

\section{FITC conjugated silica nanoparticles}

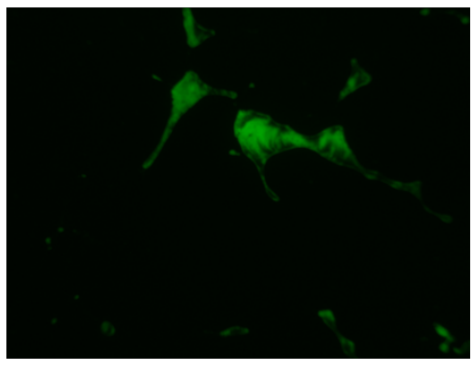

$10 \min$
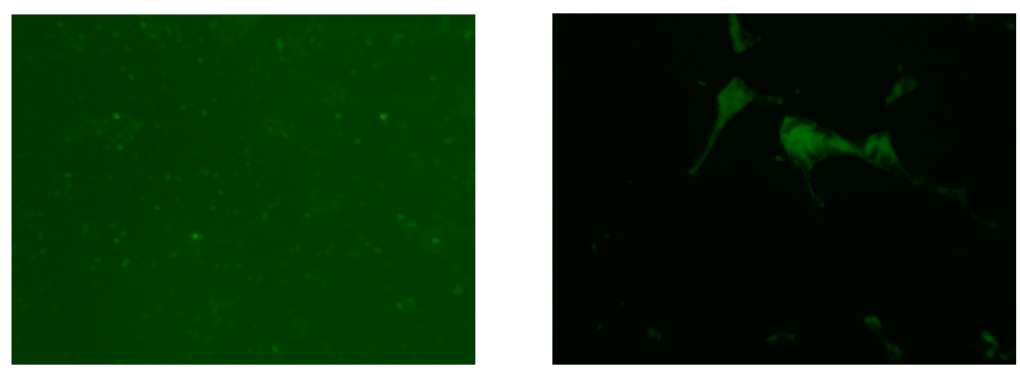

$20 \min$
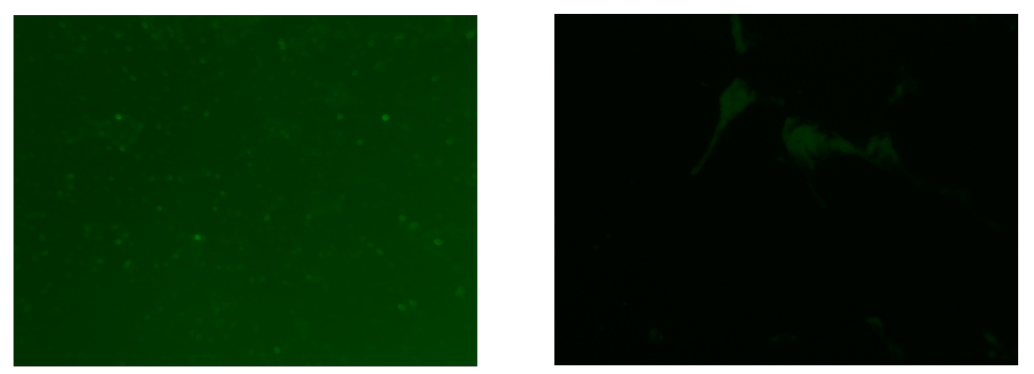

$30 \min$
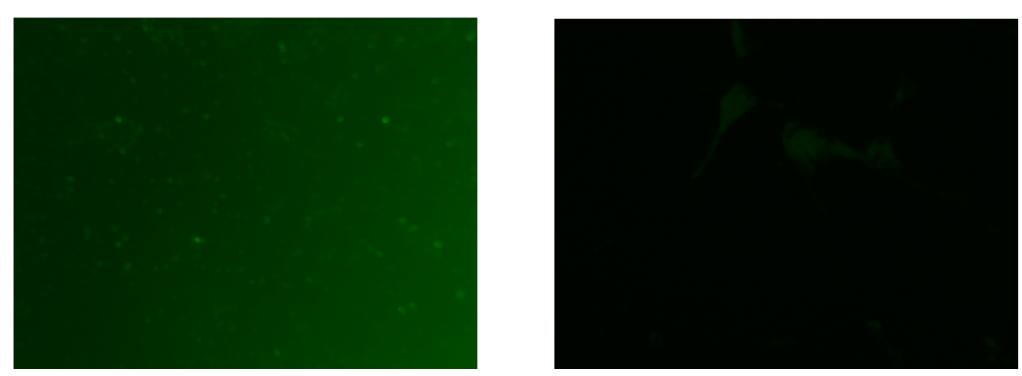

Figure 6-13: Photo images of free fluorescein-5-isothiocyanate (FITC) and fluorescein-5-isothiocyanate (FITC) conjugated silica nanoparticles from 0 to 30 minutes by 10 minutes interval

As may be seen in Figure 6-13, the fluorescence life time of free fluorescein-5isothiocyanate (FITC) and fluorescein-5-isothiocyanate (FITC) conjugated silica nanoparticles at same volume did not show better photo-stability compared to quantum dots such as cadmium selenide (CdSe) quantum dots. ${ }^{26}$ Intensity of the brightness was measured and the result was shown in Figure 6-14. From the graph, 
photo-bleaching of the fluorescein-5-isothiocyanate (FITC) conjugated silica nanoparticles was even faster than free fluorescein-5-isothiocyanate (FITC). It could be due to the amount of fluorescein-5-isothiocyanate (FITC). Since silica nanoparticles have larger capacity than free fluorescein-5-isothiocyanate (FITC), the amount of fluorescein-5-isothiocyanate (FITC) of fluorescein-5-isothiocyanate (FITC) conjugated silica nanoparticles will be reduced compared to free fluorescein-5isothiocyanate (FITC) in the same volume.

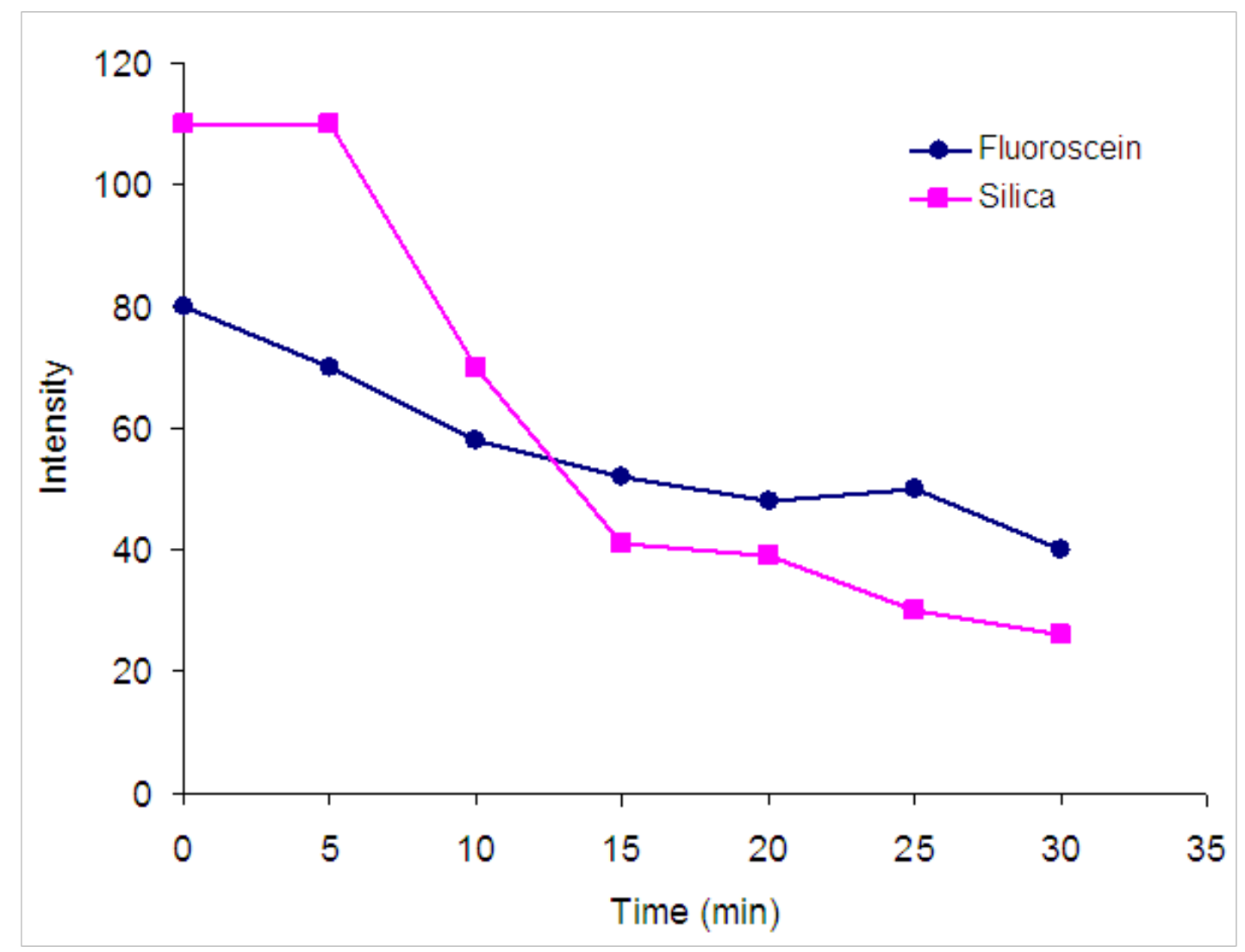

Figure 6-14: Time dependent brightness intensity of free fluorescein-5-isothiocyanate (FITC) and fluorescein-5-isothiocyanate (FITC) conjugated silica nanoparticles 


\section{FTIR analysis}

To characterise the surface molecules of the silica nanoparticles conjugated with fluorescein-5-isothiocyanate (FITC), the nanoparticles were analysed by FTIR spectroscopy. The spectrum is shown in

Figure 6-15.

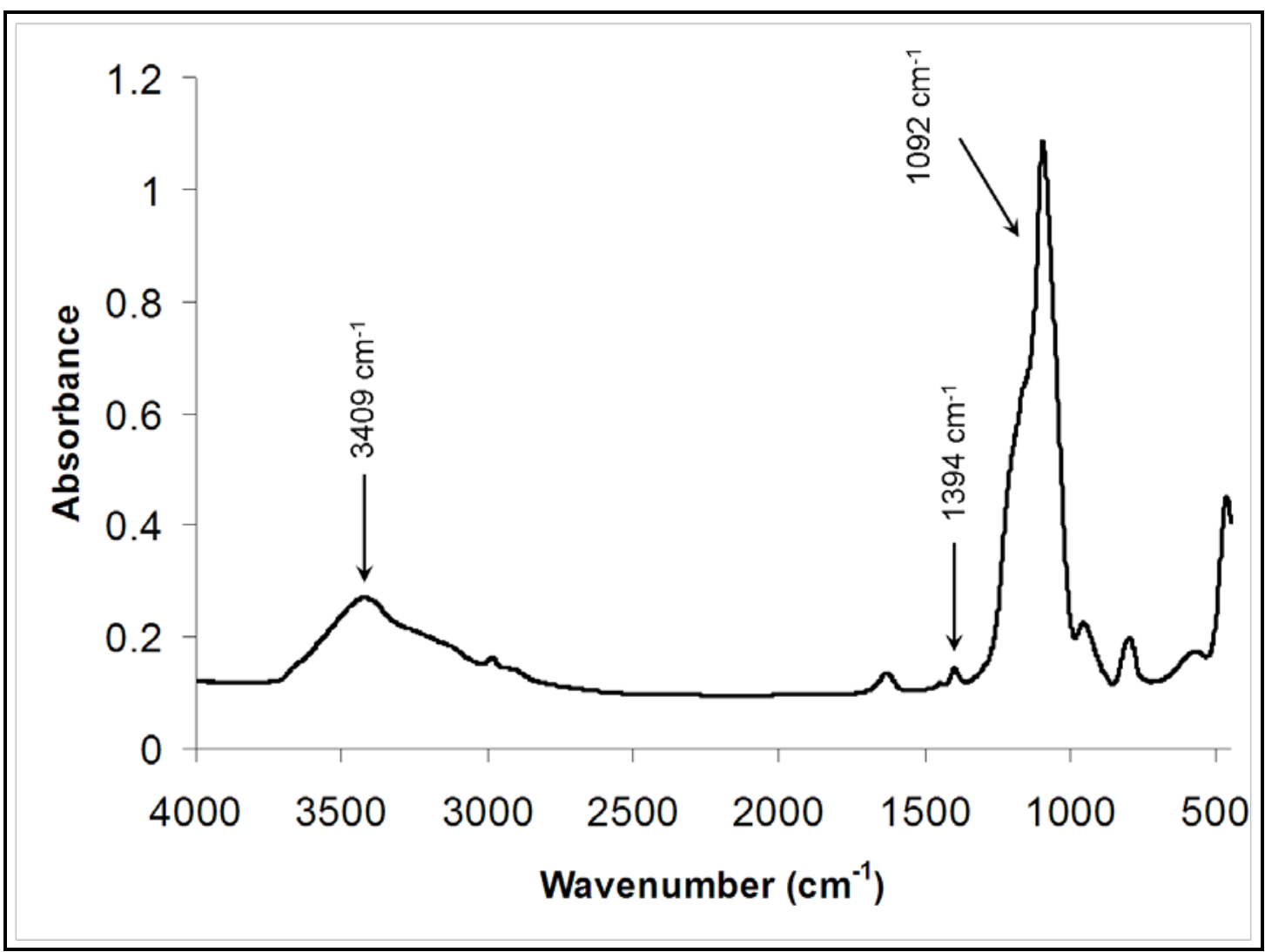

Figure 6-14: FTIR spectrum of silica nanoparticles conjugated with fluorescein-5isothiocyanate (FITC)

In this FTIR spectrum, O-H stretching from the hydroxyl groups on the surface of the nanoparticles can be clearly seen at $3409 \mathrm{~cm}^{-1}$. Also O-H in plane bending can be seen at $1394 \mathrm{~cm}^{-1}$. A very strong Si-O stretching can be seen at $1092 \mathrm{~cm}^{-1}$ which indicates presence of silica nanoparticles. The result shows the surface molecules of the silica nanoparticles is hydrophilic.

\section{Eye Test}

The behaviour of the fluorescein-5-isothiocyanate (FITC) conjugated silica nanoparticles in a human eye was observed by our collaborators in University of 
Western Sydney (Dr. Santosh Khanal, and Prof. Thomas Millar). A drop of the fluorescein-5-isothiocyanate (FITC) conjugated silica nanoparticles in ethanol and observed their behaviour in the eye by monitoring the fluorescence of the nanoparticles. The fluorescence was monitored by an optical microscope with the excitation wavelength of $360 \mathrm{~nm}$ to $400 \mathrm{~nm}$ which is the ultra-violet region cause no harm in eyes. The images are shown in Figure 6-16 and Figure 6-17.

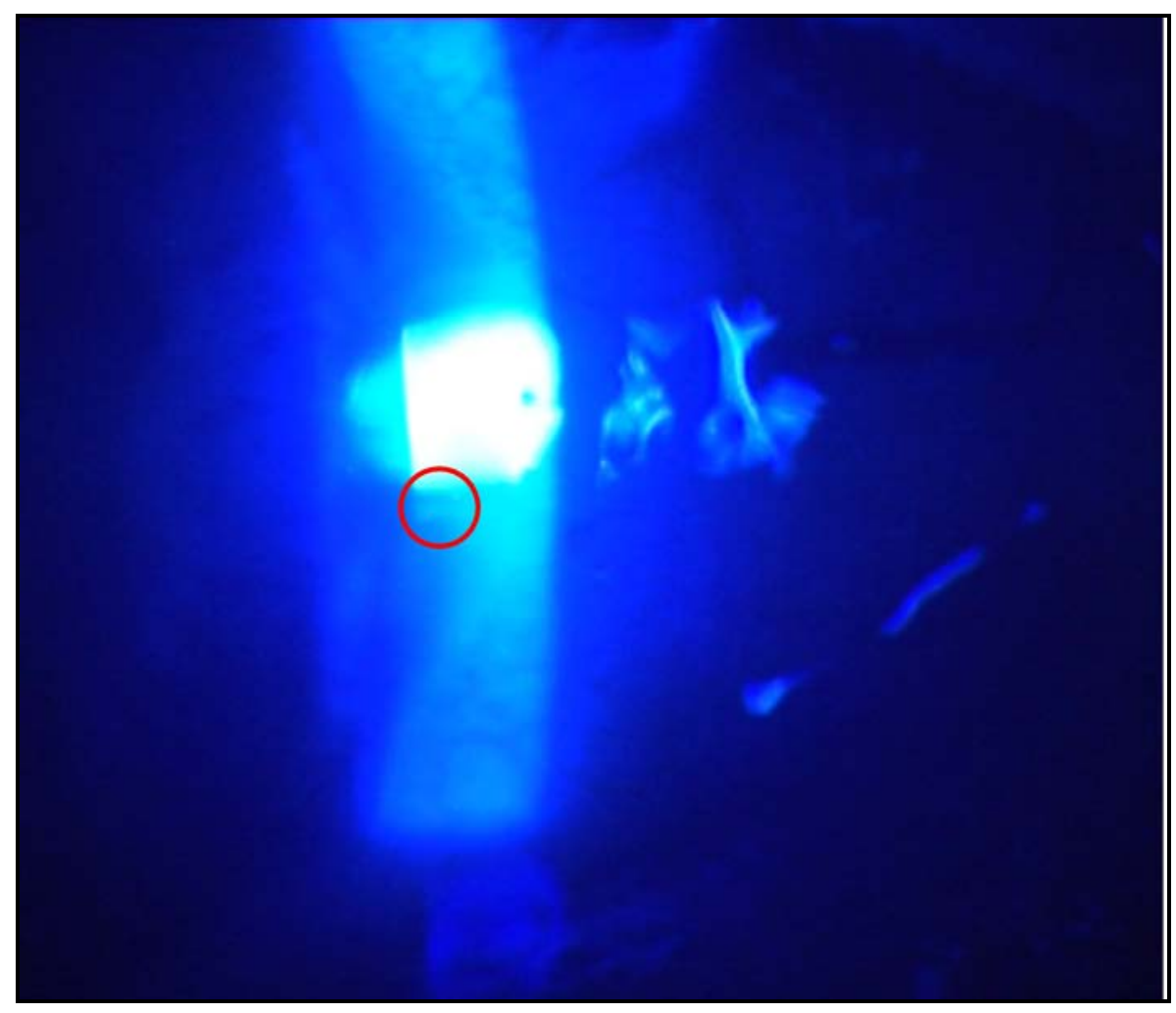

Figure 6-15: Silica nanoparticles conjugated with fluorescein-5-isothiocyanate (FITC) on an eye surface. The light green colour inside the red circle shows fluorescence from the nanoparticles 


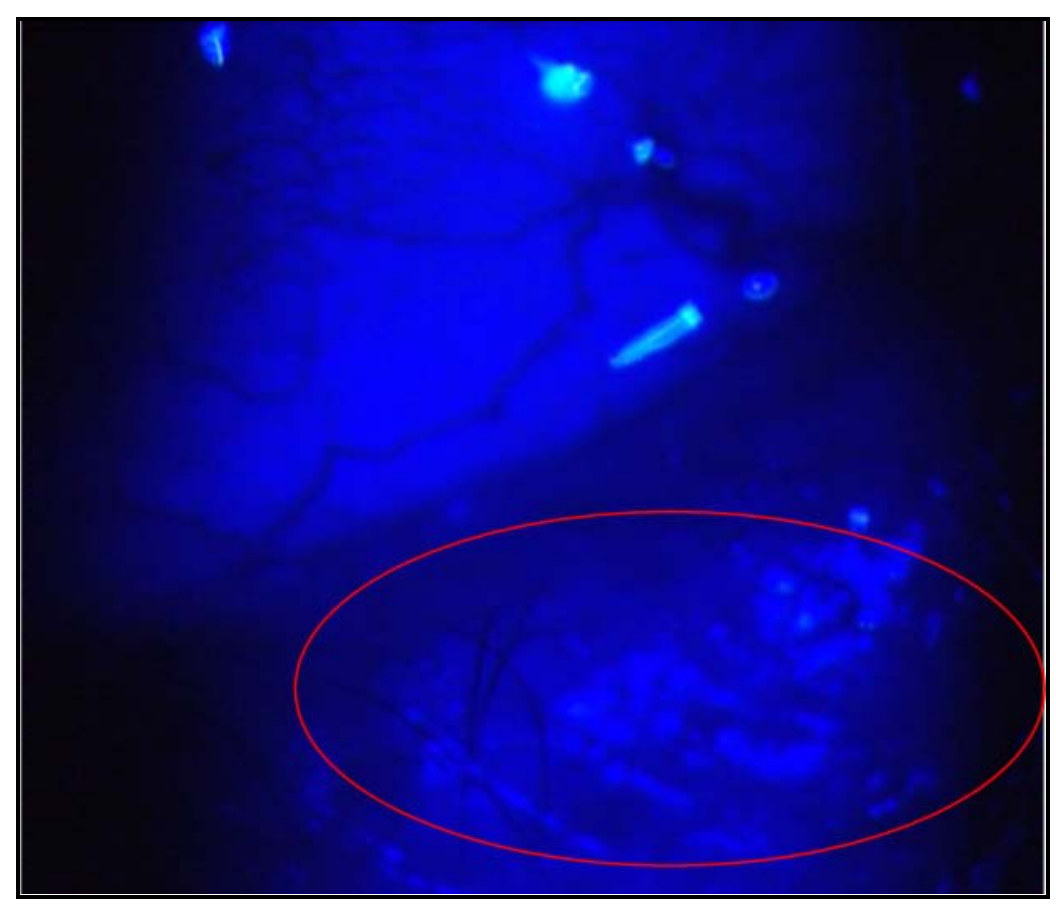

Figure 6-16: Silica nanoparticles conjugated with fluorescein-5-isothiocyanate (FITC) on the eye lid. The light green fluorescence inside the red oval is from the silica nanoparticles on the eye lid.

The silica nanoparticles conjugated with fluorescein-5-isothiocyanate (FITC) synthesised in experiment 5 were applied on a human eye as a tear film marker. The green fluorescence from fluorescein-5-isothiocyanate (FITC) was observed clearly on the eye. The nanoparticles moved upward with each blink which is very typical behaviour of hydrophobic molecules. Also the fluorescence pattern in Figure 6-17 showed typical hydrophobic tear film behaviour. ${ }^{27}$ Since no surface modification was applied on the nanoparticles in experiment 5 the surface molecules of the silica nanoparticles are most likely to have hydroxyl groups. However from the eye test experiment the silica nanoparticles showed behaviour of hydrophobic molecules. It is possibly due to the size effect. Since the silica nanoparticles were large enough to precipitate out in ethanol or water they may not be dispersed in aqueous layer of the tear film. 


\section{6-2. Summary and Discussion}

Organic dye doped silica nanoparticles were synthesised and characterised by SEM, DLS and optical properties were measured. Several organic dyes were used to dope silica nanoparticles, however only fluorescein-5-isothiocyanate showed the evidence of encapsulation.

The failure of experiments 1 to 4 is possibly due to lack of the formation of a covalent bond between the dye molecules and the silica matrix. It was expected rhodamine would bind to silica nanoparticles through an electrostatic attraction due to the positive charge on the nitrogen. However it was not strong enough to keep the dye on the surface of the nanoparticles and dye came off during the purification process. ${ }^{20}$ The failure of the experiments 1 to 4 indicates that electrostatic force attraction is not suitable to keep the dye in silica nanoparticles but needs a covalent bond. This result corresponds to the one reported by Santra et. al. ${ }^{28}$

Tetraethoxysilane (TEOS) requires a strong nucleophile such as $\mathrm{OH}^{-}$to form a covalent bond. For experiment 3 and 4, the carboxyl group of free fluorescein was expected to form covalent bond between the silica matrix however, the results showed no evidence of this. This is possibly because the carboxylate anion is not strong enough to form a covalent bond with the silica matrix. Also steric hindrance could prevent the dye molecule from attaching.

From the SEM image of experiment 5, mono dispersed spherical shape nanoparticles were observed with an average size of $127.7 \mathrm{~nm}$ which are much larger size compared to other quantum dots such as silicon or germanium quantum dots. In terms of nanoparticle size control, there is a significant difference between the synthesis systems of silicon or germanium quantum dots and silica nanoparticles. Nanoparticle size for silicon or germanium quantum dots is controlled by size of the micelles which is related to the type of surfactant or molar ratio of the precursor and surfactant. On the other hand, the reaction system of the silica nanopaticles is based on the hydrolysis reaction of tetraethoxysilane (TEOS) in which the size can be controlled by molar ratio of precursor and solvent and also reaction temperature. ${ }^{31,32}$

From optical properties, free fluorescein-5-isothiocyanate (FITC) molecules showed about $30 \mathrm{~nm}$ red-shifted emission peak compared to fluorescein-5-isothiocyanate (FITC) conjugated silica nanoparticles. This is probably because isothiocyanate group 
formed a bond with the amine group of 3-amino-propyltriethoxylsilane (APTES) and the molecular orbital of the fluorescein-5-isothiocyanate (FITC) was affected. ${ }^{30}$ The emission peaks of fluorescein-5-isothiocyanate (FITC) doped silica nanoparticles are independent of the excitation wavelengths. This allows simultaneous imaging together with other dyes at a single excitation wavelength. On the other hand, free fluorescein5-isothiocyanate (FITC) showed wide emission shifts dependent on the excitation wavelength.

In the excitation spectra, the peaks of the fluorescein-5-isothiocyanate (FITC) conjugated silica nanoparticles from $250 \mathrm{~nm}$ to $350 \mathrm{~nm}$ were sharper compared to the excitation spectrum of free fluorescein-5-isothiocyanate (FITC). It could be due to energy transition between fluorescein-5-isothiocyanate (FITC) and silica nanoparticles without emitting photons and just a narrow region of the excitation wavelengths cause the photoluminescence. Fluorescence of $20 \mu \mathrm{L}$ fluorescein-5isothiocyanate (FITC) conjugated silica nanoparticles showed photo-bleaching after $10 \mathrm{~min}$ and it was faster than free fluorescein-5-isothiocyanate (FITC) molecules. Two possible reasons are listed. One could be due to mole quantity. The mole quantity of free fluorescein-5-isothiocyanate (FITC) in $20 \mu \mathrm{L}$ is much larger than the one of fluorescein-5-isothiocyanate (FITC) molecules in fluorescein-5-isothiocyanate (FITC) conjugated silica nanoparticles. Second reason could be because diffusion of free fluorescein-5-isothiocyanate (FITC) molecules. Since free fluorescein-5isothiocyanate (FITC) molecules are much lighter than silica nanoparticles diffusion rate is higher than silica nanoparticles and it probably affected fluorescence life time.

From the results of this chapter, fluorescein doped silica nanoparticles were successfully synthesised and their optical properties are still suitable as a tear film marker for multiple imaging. The success of this experiment leads the next step which is surface modification of these silica nanoparticles. Since free fluorescein is water soluble it cannot be used as a lipid layer marker. However, by surface modification on these silica nanoparticles to make them hydrophobic or hydrophilic as future work will make them possible to use them as a lipid layer marker of tear film. Also rose Bengal as a pink fluorescent dye being widely used in human eyes can be next target to encapsulate in the silica nanoparticles. 


\section{6-3. References}

1. M. I. Roat, E. Romanowski, T. Araullo-Cruz, Y. J. Gordon; Arch OPhthalmol., 1987, 105, 1415-1417, “The Antiviral Effects of Rose Bengal and Fluorescein”

2. http://www.allaboutvision.com/conditions/dryeye.htm

3. http://arthritis.about.com/od/sjogrens/a/guidetodryeyes 2.htm

4. http://www.docshop.com/education/vision/eye-disorders/dry-eye/diagnosis/

5. http://en.wikipedia.org/wiki/Fluorescein

6. A. S. Kwan, C. Barry, I. L. McAllister, I. Constable; Clin. Experiment. Ophthalmol., 2006, 34, 33-8, "Fluorescein angiography and adverse drug reactions revisited: the Lions Eye experience”

7. http://www.rnib.org.uk/eyehealth/eyeconditions/eyeconditionsdn/Pages/dry e ye.aspx

8. A. J. Bron BSc, FRCOphth; Survey of ophthalmology, 2001, 45, S221-S226, "Diagnosis of Dry Eye"

9. S. Khanal, A. Tomlinson, A. McFadyen, C. Diaper, and K. Ramaesh; IOVS, 2008, 49, 1407-1414, “Dry Eye Diagnosis”

10. A. M. Derfus, W. C. W. Chan, S. N. Bhatia; Nano Lett., 2004, 4, 11-18, "Probing the Cytotoxicity of Semiconductor Quantum Dots"

11. D. Ma, A. J. Kell, S. Tan, Z. J. Jakubek, and B. Simard; J. Phys. Chem. C, 2009, 113, 15974-15981, "Photophysical Properties of Dye-Doped Silica Nanoparticles Bearing Different Types of Dye-Silica Interactions” 
12. W. Yang, C. G. Zhang, H. Y. Qu, H. H. Yang, and J. G. Xu; Anal. Chim. Acta, 2004, 203, 163-169, "Novel fluorescent silica nanoparticle probe for ultrasensitive immunoassays"

13. H. H. Yang, H. Y. Qu, P. Lin, S. H. Li, M. T. Ding, and J. G. Xu; The Analyst, 2003, 128, 462-466, "Nanometer fluorescent hybrid silica particle as ultrasensitive and photostable biological labels”

14. J. Wang, W. B. White, and J. H. Adair; J. Phys. Chem. B., 2006, 110, 46794685, "Dispersion of SiO2-Based Nanocomposites with High Performance Liquid Chromatography”

15. L. Latterini, and M. Amelia; Langmuir, 2009, 25, 4767-4773, "Sensing Proteins with Luminescent Silica Nanoparticles”

16. S. Kim, T. Y. Ohulchanskyy, A. Baev, and P. N. Prasad; J. Mater. Chem., 2009, 19, 3181-3188, "Synthesis and nanoparticle encapsulation of 3,5difuranylvinyl-boradiaza-s-indacenes for near-infrares fluorescence imaging”

17. E. Herz, T. Marchincin, L. Connelly, D. Bonner, A. Burns, S. Switalski, and U. Wiesner; J. Fluoresc., 2010, 20, 67-72, "Relative Quantum Yield Measurements of Coumarin Encapsulated in Core-Shell Silica Nanoparticle”

18. T. Doussineau, S. Trupp, G. J. Mohr; J. Colloid Interface Sci., 2009, 339, 266270, "Ratiometric pH-nanosensors based on rhodamine-doped silica nanoparticles functionalized with a naphthalimide derivative”

19. R. P. Bagwe, C. Yang, L. R. Hilliard, and W. Tan; Langmuir, 2004, 20, 83368342, “Optimization of Dye-Doped Silica Nanoparticles Prepared Using a Reverse Microemulsion Method” 
20. K. S. Rao, K. El-Hami, T. Kodaki, K. Matsushige, and K. Makino; J. Colloid. Interf. Sci., 2005, 289, 125-131, “A novel method for synthesis of silica nanoparticles”

21. S. Santra, P. Zhang, K. Wang, R. Tapec, and Weihong Tan; Anal. Chem. 2001, 73, 4988-4993, “Conjugation of Biomolecules with Luminophore-Doped Silica Nanoparticles for Photostable Biomarkers”

22. Y. S. Lin, S. H. Wu, C. T. Tseng, Y. Hung, C. Chang, and C. Y. Mou; Chem. Commun., 2009, 24, 3542-3544, "Synthesis of hollow silica nanospheres with a microemulsion as the template”

23. H.Kamiya: http://mandala.t.u-tokyo.ac.jp/modules/knw/ppt/d304.pdf, Tokyo University of Agriculture and Technology, "ナノ粒子の分散、凝集制御”

24. S. I. Mian, A. Y. Li, S. Dutta, D. C. Musch, R.M. Shtein; J. Cataract. Refract. Surg., 2009, 35, 2092-2098, “ Dry eyes and corneal sensation after laser in situ keratomileusis with femtosecond laser flap creation: Effect of hinge position, hinge angle, and flap thickness”

25. A. J. Bron; Surv. Ophthalmol., 2001, 45, S221-S226, “ Diagnosis of Dry Eye”

26. Y. H. Sun, Y. S. Liu, P. T. Vernier, C. H. Liang, S. Y. Chong, L. Marcu, and M. A. Gundersen; Nanotechnol., 2006, 17, 4469-4476, "Photostability and pH sensitivity of CdSe/ZnSe/ZnS quantum dots in living cells”

27. http://www.clspectrum.com/article.aspx?article=11713

28. S. Santra, B. Liesenfeld, C. Bertolino, D. Dutta, Z. Cao, W. Tan, B. M. Moudgil, and R. A. Mericle; J. Lumin., 2006, 117, 75-82, "Fluorescence lifetime measurments to determine the core-shell nanostructure of FITC-doped silica nanoparticles: An optical approach to evaluate nanoparticle photostability" 
29. http://www.pharmaziestudenten-hd.de/analytik/uv-vis2/entheorie_frame.html

30. D. Gao, Z. Wang, B. Liu, L. Ni, M. Wu, and Z. Zhang; Anal. Chem., 2008, 80, 8545-8553, "Resonance Energy Transfer-Amplifying Fluorescence Quenching at the Surface of Silica Nanoparticles toward Ultrasensitive Detection”

31. N. El. Hawi, C. Nayral, F. Delpech, Y. Coppel, A. Cornejo, A. Castel, and B. Chaudret; Langmuir, 2009, 25, 7540-7546, “Silica Nanoparticles Grown and Stabilized in Organic Nonalcoholic Media”

32. H. C. Wang, C. Y. Wu, C. C. Chung, M. H. Lai, and T. W. Chung; Ind. Eng. Chem. Res., 2006, 45, 8043-8048, “Analysis of Parameters and Interaction between Parameters in Preparation of Uniform Silicon Dioxide Nanoparticles Using Response Surface Methodology” 


\section{Chapter 7 Overall conclusions and future}

\section{work}

This research has focused on the synthesis and surface modification of silicon and germanium quantum dots for biological applications. Also, the synthesis of silica nanoparticles for dry eye diagnosis purposes was described. Syntheses of silicon quantum dots have been explored over a few years. ${ }^{1-7}$ However research on the surface chemistry of silicon quantum dots has not made remarkable progress. One of the main purposes of this thesis is to attach bio-molecules to the surface of the silicon quantum dots and purify them to be safe to use in vitro.

A larger variety of capping molecules were attached onto the surface of silicon and germanium quantum dots compared to those quantum dots in literature reports. These quantum dots were characterised by TEM, SAED, EDS, FTIR, and NMR in order to establish the knowledge about size and shape distributions, crystal structures, chemical compositions, and the surface molecules of the quantum dots. Also, the optical properties of the capped silicon quantum dots were measured and cell viability assays were conducted to assess the possibility of their biological use.

A brief summary and future work to improve the research work of each particular chapter are described in the sections below.

\section{7-1. Synthesis and surface modification of silicon quantum dots}

The surface modified silicon quantum dots were synthesised by microemulsion system with strong reducing agents such as $\mathrm{LiAlH}_{4}$ or $\mathrm{LiBH}_{4}$. The surface modification was conducted on the hydrogen terminated silicon quantum dots. A variety of organic capping molecules were applied to attach onto the surface of the silicon quantum dots including hydrophobic and hydrophilic molecules. The surface molecules were characterised mainly by FTIR and ${ }^{1} \mathrm{H}$ NMR analysis. Most of the 
experiments showed evidence of the attachment of the capping molecules on the surface of silicon quantum dots. However some molecules which have a terminal hydroxyl group or carboxyl group did not give spectra in which peaks of the functional groups can be seen. It was probably due to the oxygen atomsbinding to silicon atoms on the surface. This was the motivation of further work described in chapter 4 .

Future work on this chapter will include more specific characterisation of the surface molecules, since ${ }^{1} \mathrm{H}$ NMR analysis shows the state of organic molecules but not evidence of a direct bond between silicon and carbon atoms. Therefore by taking ${ }^{29} \mathrm{Si}$ NMR, bonding of capping molecules and silicon atoms on the surface can be detected. Furthermore diffusion NMR analysis can characterise the speed of diffusion of capping molecules. The diffusion speed of free capping molecules should be much faster than the ones attached on the surface of the quantum dots. This could be a key point to determine the attachment of capping molecules on the surface of the quantum dots. In terms of biological use, there still a room to improve the purification system. Since most of the chemicals used in the syntheses were harmful, corrosive or toxic, they need to be completely removed before the quantum dots are applied in biological conditions. From the purification results, about $70 \%$ to $99 \%$ surfactant molecules were removed after size exclusion column chromatography and about $90 \%$ was removed by liquid-phase separation. However the results showed the efficiency of the column chromatography is not always constant. There are not many reports presenting purification techniques of quantum dots however, as introduced in chapter 1, dialysis purification has potential to be applied for silicon quantum dots.

\section{7-2. Chemical reactions on the surface of silicon quantum dots and its biological application}

Due to the failure to attach functional molecules which have terminal carboxyl group or hydroxyl groups, chemical reactions on the terminal carbon double bonds on the surface of the silicon quantum dots have been conducted. The main concern regarding the trial of capping with diene molecules was the possibility of forming 
bridges between nanoparticles, however from ${ }^{1} \mathrm{H}$ NMR analysis the result showed the existence of a terminal double bond on the surface of the silicon quantum dots. The reactions converting the carbon-carbon double bond to a hydroxyl group, epoxide group or diol group were successful, as assessed by ${ }^{1} \mathrm{H}$ NMR spectra ${ }^{8}$ however Jones oxidation $^{9}$ on the terminal hydroxyl group to form carboxyl group did not succeed. Since the Jones oxidation requires strong acids such as sulphuric acid and a chromium (VI) compound which is very toxic to organs, it might be better to apply different ways to make carboxyl groups. As alternative options, converting an alkyl halide by using Grignard reagent or sodium cyanide can be applied for future plans. ${ }^{10}$

Grignard reagent reacts with carbonyl carbon of the carbon dioxide and it gives carboxyl anion. By treating this carboxyl ion with strong acid such as hydrochloric acid it gives a carboxylic acid. The reaction scheme is given below.

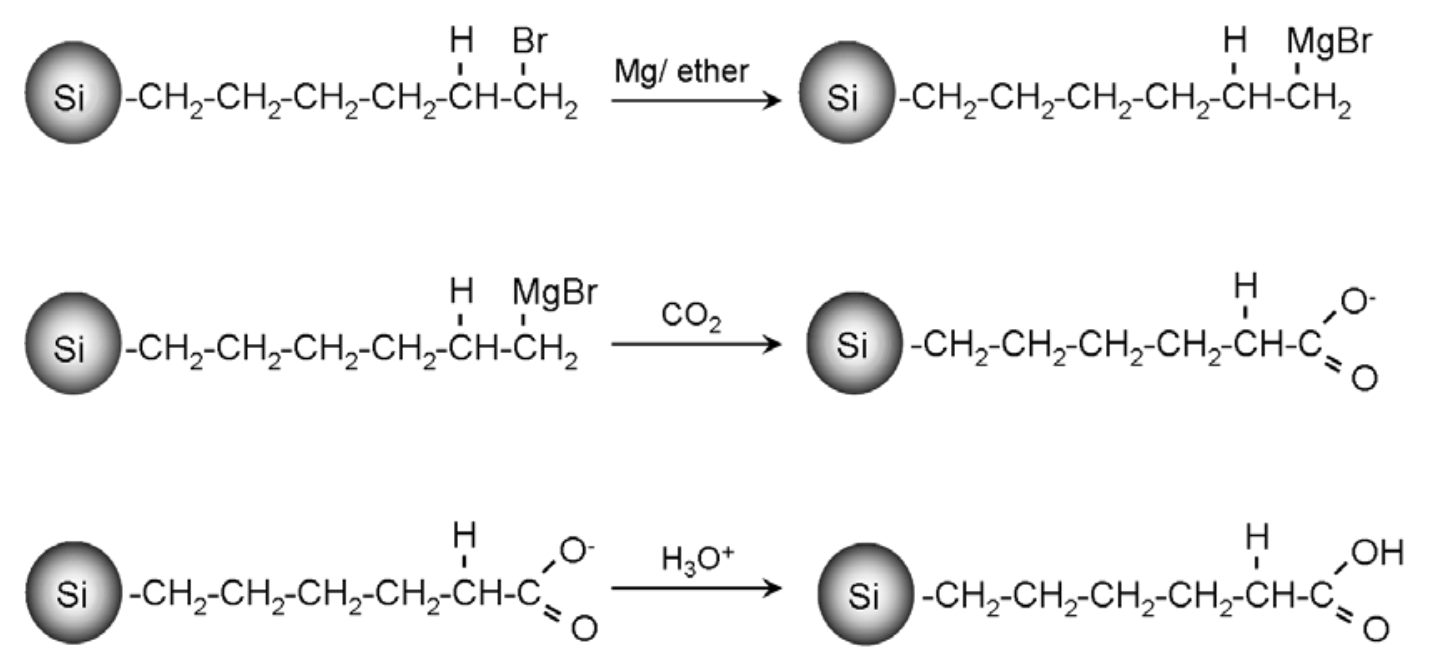

Reaction scheme of forming carboxyl group by using Grignard reagent

For the second option, a primary alkyl halide can react with sodium cyanide. ${ }^{10}$ The cyanide ion will replace the halogen and this makes a new carbon-carbon bond. Its carbon-nitrogen triple bond can be hydrolyzed with aqueous media such as hydrochloric acid to form carboxylic acid. The reaction scheme is given below. 


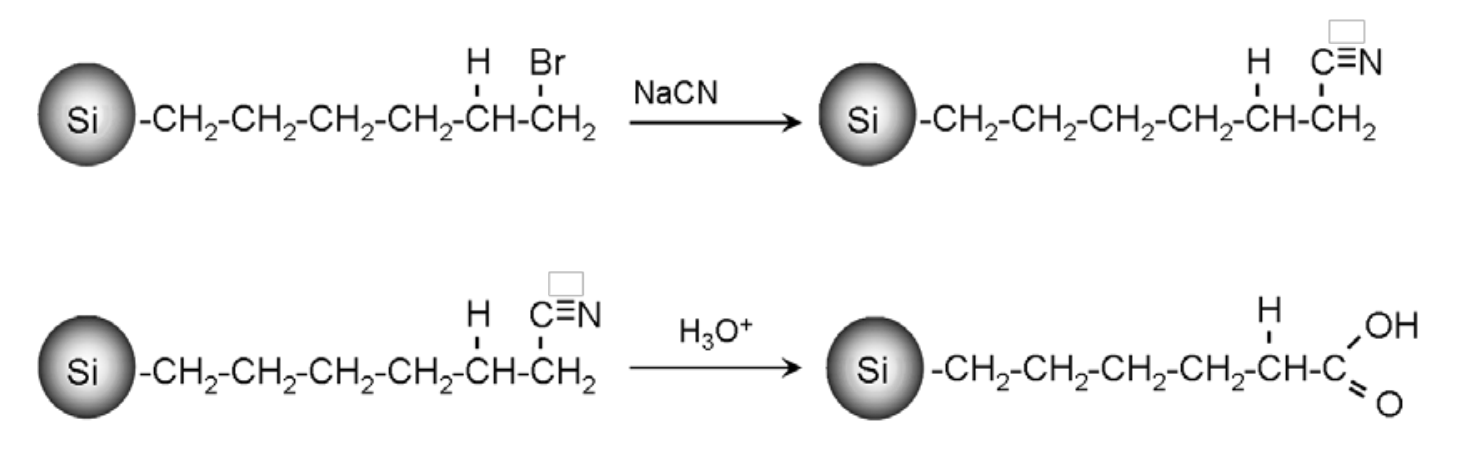

\section{Reaction scheme of forming carboxyl group by using sodium cyanide}

Another task for future work is to solve the quenching problem. When large biomolecules are attached to silicon quantum dots there is the possibility of losing fluorescence by quenching. To prevent silicon quantum dots from quenching, coating them with a shell may have possibilities. Jing et. al reported that fluorescence quantum yield of CdTe quantum dots increased by coating with a silica shell. ${ }^{11,12}$ Also Talapin et. al synthesised CdSe quantum dots with a ZnS shell which have better emission intensity compared to the ones without the shell. ${ }^{13}$ Since silicon atom forms covalent bonds with germanium, a germanium shell with silicon core could be an option for future work.

\section{7-3. Syntheses of germanium quantum dots}

Two approaches were taken to synthesise germanium quantum dots for biological applications. One approach was to use a micro emulsion system and other one was to use a high temperature bench top synthesis. All the samples were characterised by TEM. Relatively mono dispersed spherical nanoparticles were observed in most of the samples obtained from the high temperature bench top synthesis. The surface molecules of most of the nanoparticles from high temperature bench top synthesis were characterised by FTIR analysis. The samples obtained from microemulsion synthesis did not show promising data on TEM. Since germanium is heavier than silicon the nanoparticles precipitated. During synthesis the yellow or orange precipitate was collected from the reaction mixture and washed with solvent to remove the surfactant, however the precipitate did not show any peaks from X-ray diffraction analysis. The result showed the precipitate is not crystalline germanium even though it was proved that it is composed mainly of germanium. For future work, 
combinations of different types of surfactant, solvent or precursor can be investigated to explore particle sizes or shapes.

Liu and co-workers reported the synthesis of crystalline germanium nanostructures by reducing $\mathrm{GeO}_{2}$ with $\mathrm{NaBH}_{4}$ under basic conditions. ${ }^{14}$ The reaction can be undertaken at room temperature and the structure size and shape can be controlled by changing the molar ratio of precursor and reducing agent. Aqueous ammonia can be used as a solvent. $\mathrm{NaBH}_{4}$ is a milder reductant and it can release hydrogen in alkaline aqueous medium at room temperature. Therefore due to the low safety risk, this system has large potential for the future work of the synthesis of germanium quantum dots. The report was mainly focused on the synthesis and no surface modification was mentioned, however since the produced crystalline germanium nanostructure was hydrogen terminated, there would be a wider possibility to functionalise the surface with organic molecules by using alkenes.

Solid state NMR analysis can be a good method to characterise the surface molecules of those nanoparticles. Since germanium nanoparticles have a larger size compared to silicon quantum dots and they crushed out after reactions ${ }^{1} \mathrm{H}$ NMR analysis could not be applied to characterise the surface molecules.

Even though the germanium nanoparticles from high temperature bench top synthesis showed promising data from TEM and FTIR analysis, none of the samples studied here showed photon emission in the visible region. This could be due to the size of the nanoparticles. The exciton Bohr radius of germanium is $24.3 \mathrm{~nm}$ and the average particle size of most of the samples was much larger than that. Another possible reason could be due to quenching by capping molecules. Since this synthesis system requires reaction temperature higher than $260{ }^{\circ} \mathrm{C}$, all the capping molecules applied for the experiments have relatively large molecular weights and long carbon chains therefore the possibility of quenching would be higher. For future work combinations of different reaction temperatures and reaction time can be explored to control and reduce the size of the nanoparticles.

The next step for this research could involve cyto-toxicity studies of these quantum dots. As has been done for biological applications on silicon quantum dots, toxicity studies will be necessary and investigation of cell uptake and cyto-toxicity have to be studied before going on to the study in organs. 


\section{7-4. Synthesis and characterisation of silica nanoparticles for dry eye diagnosis}

The synthesis of silica nanoparticles conjugated with an organic dye for dry eye diagnosis was conducted. The motivation of this research is due to the unsuitability of silicon quantum dots for this application because of their optical properties. To gain more red-shifted emission, the idea of encapsulating organic dye in silica nanoparticles was applied. Rhodamine doped silica nanoparticles were reported by Ma et. al. ${ }^{15}$

In this research fluorecein-5-isothiocyanate was combined with silica nanoaprticles. Rose Bengal and Rhodamine were also applied to silica nanoparticles however the samples obtained from these experiments did not show the attachment of those dyes to the nanoparticles. Fluorecein doped silica nanoparticles were very well mono dispersed in the average size of $163.7 \mathrm{~nm}$ from the results of dynamic light scattering (DLS) analysis and SEM images. The emission peak of fluorescein conjugated silica nanoparticles was slightly blue-shifted compared to the emission peak of free fluorescein-5-isothiocyanate (FITC) however it is still in the green visible region. $\mathrm{Xu}$ et. al reported longer photo-stability of Lissamine rhodamine B sulfonylchloride (LRSC) doped silica nanoparticles and FITC doped silica nanoparticles compared to the each of free organic dye molecule. ${ }^{16,17}$ However under the optical microscope, the FITC doped silica nanoparticles obtained from experiment 5 from chapter 6 did not offer photo-stability any longer than free FITC. Therefore for future work we need to overcome the photo-stability problem. Since the synthesis system is slightly different from the one from $\mathrm{Xu}$ et. $\mathrm{al}^{16,17}$ such as the precursor and no use of surfactant, it might have affected the results.

The aim of this research is to find out the interaction between different layers of tear films. Therefore the ideal way for this is to have multiple colours fluorescent silica nanoparticles and mark each layer with a different colour. In this research only fluorecein doped silica nanoparticles were synthesised. Therefore another step for future work would be doping different dyes with silica nanoparticles such as rose Bengal or Lissamine green. The structures of them are shown in Figure 7-1. 
<smiles>CN(C)c1ccc(C(=C2C=CC(=[N+](C)C)C=C2)c2c(O)c(S(=O)(=O)[O-])cc3cc(S(=O)(=O)[O-])ccc23)cc1</smiles>

Figure 7-1: (a); a chemical structure of rose Bengal (b); Lissamine green B

To mark different layers of the tear film, surface molecules of the silica nanoparticles become very important. To mark aqueous layer they need to be water soluble, yet on the other hand, hydrophobic surfaces will be required to mark the lipid layer. Xu et. al also reported some surface modification on the silica nanoparticles doped with FITC or LRSC to attach aintibodies. ${ }^{17}$ In their research, silica nanoparticles were treated with 3-aminopropyltrimethoxysilane (APTMOS) to produce amine group terminated silica nanoparticles and then this terminal amine group was treated with glutaraldehyde to form $\mathrm{N}=\mathrm{C}$ bond and aldehyde terminated silica nanoparticles. This aldehyde group was used to attach an antibody which has terminal amine group by forming $\mathrm{C}=\mathrm{N}$ bond again.

Amine terminated silica nanoparticles could be applied to mark the aqueous layer and also can be used to attach lipid molecules onto the silica nanoparticles to mark the lipid layer.

For the last step, as the germanium quantum dots, to apply these silica nanoparticles to human eyes toxicity studies are necessary. Cell viability assay and toxicity test in organs must be assessed. 


\section{7-5. References}

1. R. K. Baldwin, J. Zou, K. A. Pettigrew, G. J. Yeagle, R. D. Britt, S. M. Kauzlarich; Chem. Comm., 2006, 6, 658-660, "The preparation of a phosphorus doped silicon film from phosphorus containing silicon nanoparticles”

2. J. Zou, R. K. Baldwin, K. A. Pettigrew, S. M. Kauzlarich; Nano Lett., 2004, 4, 1181-1186, "Solution Synthesis of Ultrastable Luminescent Siloxane-Coated Silicon Nanoparticles”

3. D. S. English, L. E. Pell, Z. H. Yu, P. F. Barbara, B. A. Korgel; Nano Lett., 2002, 2, 681-685, "Size Tunable Visible Luminescence from Individual Organic Monolayer Stabilized Silicon Nanocrystal Quantum Dots”

4. J. D. Holmes, K. J. Ziegler, R. C. Doty, L. E. Pell, K. P. Johnston, B. A. Korgel; J. Am. Chem. Soc., 2001, 123, 3743-3748, "Highly Luminescent Silicon Nanocrystals with Discrete Optical Transitions”

5. J. P. Wilcoxon, G. A. Samara, P. N. Provencio; Phys. Rev. B, 1999, 60, 27042714, “Optical and electronic properties of Si nanoclusters synthesized in inverse micelles”

6. R. D. Tilley, J. H. Warner, K. Yamamoto, I. Matsui, and H. Fujimori; Chem. Commun., 2005, 14, 1833-1835, "Micro-emulsion synthesis of monodisperse surface stabilized silicon nanocrystals”

7. J. H. Warner, A. Hoshino, K. Yamamoto, R. D. Tilley; Angew, 2005, 44, 4550-4554, "Water-Soluble Photoluminescent Silicon Quantum Dots”

8. A. Shiohara, S. Hanada, S. Prabakar, K. Fujioka, T. H. Lim, K. Yamamoto, P. T. Northcote, and R. D. Tilley; J. Am. Chem. Soc., 2010, 132, 248-253, 
“Chemical Reactions on Surface Molecules Attached to Silicon Quantum Dots”

9. http://www.organic-chemistry.org/namedreactions/jones-oxidation.shtm

10. http://chemistry2.csudh.edu/rpendarvis/carbacid.html

11. Y. Yang, L. Jing, X. Yu, D. Yan, and M. Gao; Chem. Mater., 2007, 19, 41234128, "Coating Aqueous Quantum Dots with Silica via Reverse Microemulsion Method: Toward Size-Controllable and Robust Fluorescent Nanoparticles"

12. L. Jing, C. Yang, R. Qiao, M. Niu, M. Du, D. Wang, and M. Gao; Chem. Mater., 2010, 22, 420-427, “Highly Fluorescent CdTe@ $\mathrm{SiO}_{2}$ Particles Prepared via Reverse Microemulsion Method”

13. D. V. Talapin, A. L. Rogach, A. Kornowski, M. Haase, and H. Weller; Nano Lett., 2001, 1, 207-211, "Highly Luminescent Monodisperse CdSe and CdSe/ZnS Nanocrystals Synthesized in a HexadecylamineTrioctylphosphine Oxide-Trioctylphospine Mixture"

14. C. Jing, X. Zang, W. Bai, J. Chu, and A. Liu; Nanotechnol., 2009, 20, 505607, "Aqueous germanate ion solution promoted synthesis of worm-like crystallized Ge nanostructures under ambient conditions”

15. D. Ma, A. J. Kell, S. Tan, Z. J. Jakubek, and B. Simard; J. Phys. Chem. C, 2009, 113, 15974-15981, "Photophysical Properties of Dye-Doped Silica Nanoparticles Bearing Different Types of Dye-Silica Interactions”

16. H. H. Yang, H. Y. Qu, P. Lin, S. H. Li, M. T. Ding, and J. G. Xu; Analyst, 2003, 128, 462-466, "Nanometer fluorescent hybrid silica particle as ultrasensitive and photostable biological labels” 
17. W. Yang, C. G. Zhang, H. Y. Qu, H. H. Yang, and J. G. Xu; Anal. Chim. Acta., 2004, 503, 163-169, “Novel fluorescent silica nanoparticle probe for ultrasensitive immunoassays” 